\title{
Boundary Element Analysis of a Curved Tubing with a Semi-Elliptical Crack
}

\author{
by \\ Kirsten Irene Plante \\ A Thesis submitted to \\ the Faculty of Graduate Studies and Research \\ in partial fulfilment of \\ the requirements for the degree of
}

Master of Applied Science

Ottawa-Carleton Institute for

Mechanical and Aerospace Engineering

Department of Mechanical and Aerospace Engineering

Carleton University

Ottawa, Ontario, Canada

June 2008

Copyright (C)

2008 - Kirsten Irene Plante 


$\begin{array}{ll}\begin{array}{l}\text { Library and } \\ \text { Archives Canada }\end{array} & \begin{array}{l}\text { Bibliothèque et } \\ \text { Archives Canada }\end{array} \\ \begin{array}{l}\text { Published Heritage } \\ \text { Branch }\end{array} & \begin{array}{l}\text { Direction du } \\ \text { Patrimoine de l'édition }\end{array} \\ \begin{array}{l}\text { 395 Wellington Street } \\ \text { Ottawa ON K1A 0N4 } \\ \text { Canada }\end{array} & \begin{array}{l}\text { O95, rue Wellington } \\ \text { Ottawa ON K1A 0N4 } \\ \text { Canada }\end{array}\end{array}$

Your file Votre référence ISBN: 978-0-494-44056-8

Our file Notre référence

ISBN: 978-0-494-44056-8

NOTICE:

The author has granted a nonexclusive license allowing Library and Archives Canada to reproduce, publish, archive, preserve, conserve, communicate to the public by telecommunication or on the Internet, loan, distribute and sell theses worldwide, for commercial or noncommercial purposes, in microform, paper, electronic and/or any other formats.

The author retains copyright ownership and moral rights in this thesis. Neither the thesis nor substantial extracts from it may be printed or otherwise reproduced without the author's permission.
AVIS:

L'auteur a accordé une licence non exclusive permettant à la Bibliothèque et Archives Canada de reproduire, publier, archiver, sauvegarder, conserver, transmettre au public par télécommunication ou par l'Internet, prêter, distribuer et vendre des thèses partout dans le monde, à des fins commerciales ou autres, sur support microforme, papier, électronique et/ou autres formats.

L'auteur conserve la propriété du droit d'auteur et des droits moraux qui protège cette thèse. $\mathrm{Ni}$ la thèse ni des extraits substantiels de celle-ci ne doivent être imprimés ou autrement reproduits sans son autorisation.
In compliance with the Canadian

Privacy Act some supporting forms may have been removed from this thesis.

While these forms may be included in the document page count, their removal does not represent any loss of content from the thesis.
Conformément à la loi canadienne sur la protection de la vie privée, quelques formulaires secondaires ont été enlevés de cette thèse.

Bien que ces formulaires aient inclus dans la pagination, il n'y aura aucun contenu manquant.

\section{Canada}




\section{Abstract}

The three-dimensional boundary element method has been determined to be an efficient computational method for solving elastostatic problems, particularly those for cracked geometries. For this reason, it is employed for the analysis of thick-walled internally pressurized curved tubing. The stress distribution in a pipe bend is investigated with respect to bend radius ratio, radius ratio of the cross-section, and angle of curvature. Upon verifying the intrados as the most likely site for crack nucleation, independent of the geometric parameters, a semi-elliptical crack of semi-minor to semi-major axis ratio $b / a=0.8$ is introduced. Stress intensity factors are determined along the crack periphery, $\phi$, for the internal pressure case, with pressure acting on the crack faces.

Polynomial influence coefficients are computed for all instances and used to verify the stress intensity factors for the previous load case; they are shown to agree to within $2.5 \%$ difference. Influence coefficients may be combined through superposition to determine the stress intensity factor for any load case with the same cracked geometry. This is demonstrated using a representative set of models for various levels of overstrain due to partial autofrettage.

The stress factor in an uncracked internally pressurized pipe bend is observed to increase for smaller bend radii, lower radius ratios and larger angles of curvature. The effect of the longitudinal angle is minimal and becomes negligible at the larger bend radius. Once a semi-elliptical crack is introduced, with pressure acting on the crack 
faces, the normalized stress intensity factor results obtained are consistent with these observations. Furthermore, it is found that the normalized stress intensity factor is approximately constant up to $\phi=60^{\circ}$, after which it increases rapidly as the free surface is approached. 


\section{Acknowledgments}

The author would like to acknowledge her thesis supervisor, Professor C.L. Tan, for his patience and guidance throughout this work, as well as the kind assistance of Fiona Warman, Michelle Thompson and Peter Klimas. 


\section{Table of Contents}

$\begin{array}{ll}\text { Abstract } & \text { iii }\end{array}$

Acknowledgments $\quad$ v

Table of Contents vi

List of Tables $\quad$ ix

List of Figures $\quad$ xxii

Nomenclature $\quad$ xxvi

1 Introduction 1

1.1 Previous Work $\ldots \ldots \ldots \ldots \ldots$

1.2 Proposed Solution Method . . . . . . . . . . . . . . . . . 3

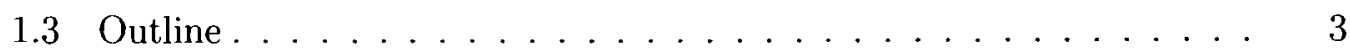

2 Review of the Boundary Element Method 5

2.1 Formulation of the BEM in Three-Dimensions . . . . . . . . . 5

2.1.1 Numerical Treatment of the BEM in Three-Dimensions . . . 8

2.1.2 Example Problem - Stress Concentrations . . . . . . . . . 10

2.2 Fracture Problems . . . . . . . . . . . . . . . 11

2.2.1 Crack-Front Boundary Elements . . . . . . . . . . 13 
2.3 Numerical Examples - Fracture Problems . . . . . . . . . . . 16

2.3.1 Circular Embedded Crack in an Infinite Solid . . . . . . 16

2.3.2 Cylinder with Semi-Elliptical Crack f . . . . . . . 17

2.4 Influence Function Method for Obtaining Stress Intensity Factors . . 19

2.4 .1 Numerical example $1 \ldots \ldots 20$

2.4 .2 Numerical example $2 \ldots \ldots \ldots 22$

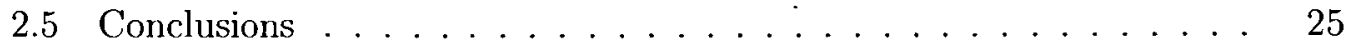

3 Stress Distributions in Pipe Bends $\quad \mathbf{5 0}$

3.1 Numerical Model . . . . . . . . . . . . . . . . . . 51

3.2 Numerical Results . . . . . . . . . . . . . . . . . . 51

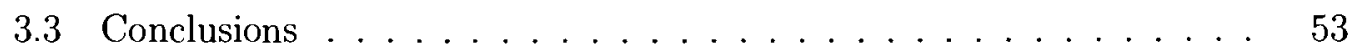

4 Stress Intensity Factors for a Crack in a Pipe Bend 68

4.1 Semi-Elliptical Crack In a Pipe Bend . . . . . . . . . . . . . . 68

4.1.1 Modeling Considerations . . . . . . . . . . . . . . . 69

4.1 .2 Mesh Refinement Study . . . . . . . . . . . 70

4.1 .3 Results......................... 71

4.2 Stress Intensity Factors from Polynomial Influence Coefficients . . . . 73

4.2 .1 Modelling Considerations . . . . . . . . . . . 73

4.2 .2 Verification ..................... 74

4.2.3 Application: Effect of Residual Stresses from Autofrettage . . 75

4.3 Conclusions . . . . . . . . . . . . . . . . . 77

$\begin{array}{lll}5 & \text { Conclusions } & 100\end{array}$

$\begin{array}{ll}\text { References } & 102\end{array}$ 
A.1 Shape Functions for Triangular Elements . . . . . . . . . . . . . . . 104

A.2 Shape Functions for Quadrilateral Elements . . . . . . . . . . 105

A.3 Shape Functions for Crack-Front Elements . . . . . . . . . . . . 106

Appendix B Stress Intensity Factors for a Crack in a Pipe Bend $\mathbf{1 1 0}$

Appendix C Influence Coefficients and Stress Intensity Factors for a

$\begin{array}{lr}\text { Crack in a Pipe Bend } & 120\end{array}$

C.1 Influence Coefficients . . . . . . . . . . . . . . . . . 120

C.2 Stress Intensity Factors from Influence Coefficients . . . . . . . . . 148

Appendix D Stress Intensity Factors due to Residual Stresses from 


\section{List of Tables}

2.1 Variation of stress concentration factor at side of circular cylindrical hole in infinite plate of finite thickness with position through the plate

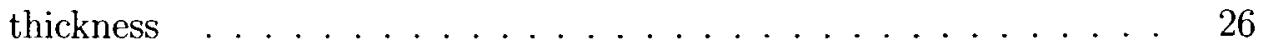

2.2 Comparison of normalized stress intensity factor, $K_{I}^{*}=K_{I} /\left(\frac{2}{\pi} \sigma \sqrt{\pi a}\right)$, for the penny-shaped crack problem . . . . . . . . . . . . . 26

2.3 Normalized influence coefficients, $K_{I}^{(i) *}=K_{I}^{(i)} /\left[A_{i}(b / W)^{i} \sqrt{\pi b}\right]$, for

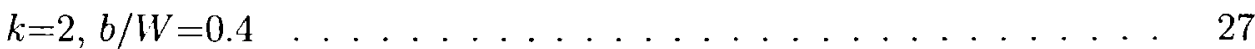

2.4 Comparison of normalized stress intensity factors, $K_{I} /(P \sqrt{\pi b})$, for $k=2, b / W=0.4 \ldots \ldots \ldots \ldots \ldots \ldots \ldots$

4.1 Corrected values of $\phi$ at nodal points on the semi-elliptical crack-front in a curved tubing $\ldots \ldots \ldots \ldots \ldots$

4.2 Normalized stress intensity factors, $K_{I} /(P \sqrt{\pi b})$, for $R^{*}=7.5, \theta=90^{\circ}$ for simple mesh refinement convergence study . . . . . . . . . .

4.3 Normalized stress intensity factors, $K_{I} /(P \sqrt{\pi b})$, at nodal points along the serni-elliptical crack-front, as defined by $\phi$, in a pressurized curved tubing for $R^{*}=5, k=2.5, \theta=90^{\circ} \ldots \ldots \ldots \ldots$

4.4 Normalized stress intensity factors, $K_{I} /(P \sqrt{\pi b})$, at nodal points along the scmi-elliptical crack-front, as defined by $\phi$, in a pressurized curved tubing for $R^{*}=5, k=2 \ldots \ldots \ldots \ldots \ldots \ldots$ 
4.5 Polynomial coefficients to define hoop stress distribution in internally pressurized uncracked curved tubing with $R^{*}=5 \ldots \ldots 83$

4.6 Polynomial coefficients to define hoop stress distribution in internally pressurized uncracked curved tubing with $R^{*}=7.5 \ldots \ldots \ldots$

4.7 Polynomial coefficients to define hoop stress distribution in internally pressurized uncracked curved tubing with $R^{*}=10 \ldots \ldots 84$

4.8 Normalized influence function coefficients, $K_{I}^{(i) *}=$ $K_{I}^{(i)} /\left[A_{i}(b / W)^{i} \sqrt{\pi b}\right]$, at nodal points along a semi-elliptical crack-front as defined by $\phi$ for $R^{*}=5, k=2, \theta=90^{\circ} \ldots \ldots \ldots \ldots$

4.9 Comparison of normalized stress intensity factors, $K_{I} /(P \sqrt{\pi b})$, obtained from the influence coefficient method and by direct BEM along crack periphery, $\phi$, for $R^{*}=5, k=2, \theta=90^{\circ} \ldots \ldots \ldots$

4.10 Comparison of normalized stress intensity factors, $K_{I} /(P \sqrt{\pi b})$, from influence coefficient method and direct BEM along crack periphery, $\phi$, for $R^{*}=10, k=1.5, \theta=90^{\circ} \ldots \ldots \ldots \ldots \ldots \ldots$

4.11 Normalized stress intensity factors, $\left(K_{I}\right)_{A} /\left[\sigma_{Y} \sqrt{\pi b}\right]$, from residual stress due to autofrettage to different levels of overstrain with $R^{*}=5$, $k=2, \theta=90^{\circ}, b / W=0.6 \ldots \ldots \ldots \ldots$

4.12 Normalized stress intensity factors, $\left(K_{I}\right)_{A} /\left[\sigma_{Y} \sqrt{\pi b}\right]$, from residual stress due to autofrettage to different levels of overstrain with $R^{*}=7.5$, $k=2, \theta=90^{\circ}, b / W=0.6 \ldots \ldots \ldots \ldots$

4.13 Normalized stress intensity factors, $\left(K_{I}\right)_{A} /\left[\sigma_{Y} \sqrt{\pi b}\right]$, from residual stress due to autofrettage to different levels of overstrain with $R^{*}=10$, $k=2, \theta=90^{\circ}, b / W=0.6 \ldots \ldots \ldots \ldots$

B.1 Normalized stress intensity factors, $K_{I} /(P \sqrt{\pi b})$, at nodal points along the semi-elliptical crack-front, as defined by $\phi$, in a pressurized curved tubing for $R^{*}=5, k=1.5$ 
B.2 Normalized stress intensity factors, $K_{I} /(P \sqrt{\pi b})$, at nodal points along the semi-elliptical crack-front, as defined by $\phi$, in a pressurized curved tubing for $R^{*}=5, k=2 \ldots \ldots \ldots \ldots \ldots$

B.3 Normalized stress intensity factors, $K_{I} /(P \sqrt{\pi b})$, at nodal points along the semi-elliptical crack-front, as defined by $\phi$, in a pressurized curved

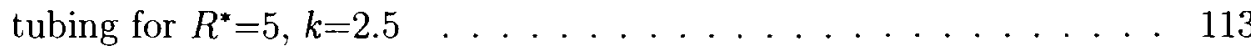

B.4 Normalized stress intensity factors, $K_{I} /(P \sqrt{\pi b})$, at nodal points along the semi-clliptical crack-front, as defined by $\phi$, in a pressurized curved tubing for $R^{*}=7.5, k=1.5$

B.5 Normalized stress intensity factors, $K_{I} /(P \sqrt{\pi b})$, at nodal points along the semi-elliptical crack-front, as defined by $\phi$, in a pressurized curved tubing for $R^{*}=7.5, k=2 \ldots \ldots \ldots \ldots \ldots \ldots$

B.6 Normalized stress intensity factors, $K_{I} /(P \sqrt{\pi b})$, at nodal points along the semi-elliptical crack-front, as defined by $\phi$, in a pressurized curved tubing for $R^{*}=7.5, k=2.5 \ldots \ldots \ldots \ldots \ldots$

B.7 Normalized stress intensity factors, $K_{I} /(P \sqrt{\pi b})$, at nodal points along the semi-elliptical crack-front, as defined by $\phi$, in a pressurized curved tubing for $R^{*}=10, k=1.5$

B.8 Normalized stress intensity factors, $K_{I} /(P \sqrt{\pi b})$, at nodal points along the semi-elliptical crack-front, as defined by $\phi$, in a pressurized curved tubing for $R^{*}=10, k=2 \ldots \ldots \ldots \ldots \ldots \ldots \ldots$

B.9 Normalized stress intensity factors, $K_{I} /(P \sqrt{\pi b})$, at nodal points along the semi-elliptical crack-front, as defined by $\phi$, in a pressurized curved tubing for $R^{*}=10, k=2.5$

C.1 Normalized influence function coefficients, $K_{I}^{(i) *}=$ $K_{I}^{(i)} /\left[A_{i}(b / W)^{i} \sqrt{\pi b}\right]$, at nodal points along a semi-elliptical crack-front as defined by $\phi$ for $R^{*}=5, k=1.5, \theta=45^{\circ}$ 
C.2 Normalized influence function coefficients, $K_{I}^{(i) *}=$ $K_{I}^{(i)} /\left[A_{i}(b / W)^{i} \sqrt{\pi b}\right]$, at nodal points along a semi-elliptical crack-front as defined by $\phi$ for $R^{*}=5, k=1.5, \theta=67.5^{\circ} \ldots \ldots \ldots \ldots$

C.3 Normalized influence function coefficients, $K_{I}^{(i) *}=$ $K_{I}^{(i)} /\left[A_{i}(b / W)^{i} \sqrt{\pi b}\right]$, at nodal points along a semi-elliptical crack-front as defined by $\phi$ for $R^{*}=5, k=1.5, \theta=90^{\circ} \ldots \ldots \ldots \ldots$

C.4 Normalized influence function coefficients, $K_{I}^{(i) *}=$ $K_{I}^{(i)} /\left[A_{i}(b / W)^{i} \sqrt{\pi b}\right]$, at nodal points along a semi-elliptical crack-front as defined by $\phi$ for $R^{*}=5, k=2, \theta=45^{\circ} \ldots \ldots \ldots \ldots$

C.5 Normalized influence function coefficients, $K_{I}^{(i) *}=$ $K_{I}^{(i)} /\left[A_{i}(b / W)^{i} \sqrt{\pi b}\right]$, at nodal points along a semi-elliptical crack-front as defined by $\phi$ for $R^{*}=5, k=2, \theta=67.5^{\circ} \ldots \ldots \ldots \ldots$

C.6 Normalized influence function coefficients, $K_{I}^{(i) *}=$ $K_{I}^{(i)} /\left[A_{i}(b / W)^{i} \sqrt{\pi b}\right]$, at nodal points along a semi-elliptical crack-front as defined by $\phi$ for $R^{*}=5, k=2, \theta=90^{\circ} \ldots \ldots \ldots \ldots \ldots$

C.7 Normalized influence function coefficients. $K_{I}^{(i) *}=$ $K_{I}^{(i)} /\left[A_{i}(b / W)^{i} \sqrt{\pi b}\right]$, at nodal points along a semi-elliptical crack-front as defined by $\phi$ for $R^{*}=5, k=2.5, \theta=45^{\circ} \ldots \ldots \ldots \ldots$

C.8 Normalized influence function coefficients, $K_{I}^{(i) *}=$ $K_{I}^{(i)} /\left[A_{i}(b / W)^{i} \sqrt{\pi b}\right]$, at nodal points along a semi-elliptical crack-front as defined by $\phi$ for $R^{*}=5, k=2.5, \theta=67.5^{\circ} \ldots \ldots \ldots \ldots$

C.9 Normalized influence function coefficients, $K_{I}^{(i) *}=$ $K_{I}^{(i)} /\left[A_{i}(b / W)^{i} \sqrt{\pi b}\right]$, at nodal points along a semi-elliptical crack-front as defined by $\phi$ for $R^{*}=5, k=2.5, \theta=90^{\circ} \ldots \ldots \ldots \ldots$

C.10 Normalized influence function coefficients. $K_{I}^{(i) *}=$ $K_{I}^{(i)} /\left[A_{i}(b / W)^{i} \sqrt{\pi b}\right]$, at nodal points along a semi-elliptical crack-front as defined by $\phi$ for $R^{*}=7.5, k=1.5, \theta=45^{\circ} \ldots \ldots \ldots$ 
C.11 Normalized influence function cocfficients, $K_{I}^{(i) *}=$ $K_{I}^{(i)} /\left[A_{i}(b / W)^{i} \sqrt{\pi b}\right]$, at nodal points along a semi-elliptical crack-front as defined by $\phi$ for $R^{*}=7.5, k=1.5, \theta=67.5^{\circ} \ldots \ldots \ldots 13$

C.12 Normalized influence function cocfficients, $K_{I}^{(i) *}=$ $K_{I}^{(i)} /\left[A_{i}(b / W)^{i} \sqrt{\pi b}\right]$, at nodal points along a semi-elliptical crack-front as defined by $\phi$ for $R^{*}=7.5, k=1.5, \theta=90^{\circ} \ldots \ldots \ldots \ldots$

C.13 Normalized influence function coefficients, $K_{I}^{(i) *}=$ $K_{I}^{(i)} /\left[\Lambda_{i}(b / W)^{i} \sqrt{\pi b}\right]$, at nodal points along a semi-elliptical crack-front as defined by $\phi$ for $R^{*}=7.5, k=2, \theta=45^{\circ} \ldots \ldots \ldots \ldots$

C.14 Normalized influence function coefficients, $K_{I}^{(i) *}=$ $K_{I}^{(i)} /\left[A_{i}(b / W)^{i} \sqrt{\pi b}\right]$, at nodal points along a semi-elliptical crack-front as defined by $\phi$ for $R^{*}=7.5, k=2, \theta=67.5^{\circ} \ldots \ldots \ldots 134$

C.15 Normalized influence function coefficients, $K_{I}^{(i) *}=$ $K_{I}^{(i)} /\left[A_{i}(b / W)^{i} \sqrt{\pi b}\right]$, at nodal points along a semi-elliptical crack-front as defincd by $\phi$ for $R^{*}=7.5, k=2, \theta=90^{\circ} \ldots \ldots \ldots$

C.16 Normalized influence function coefficients, $K_{I}^{(i) *}=$ $K_{I}^{(i)} /\left[A_{i}(b / W)^{i} \sqrt{\pi b}\right]$, at nodal points along a semi-elliptical crack-front as defined by $\phi$ for $R^{*}=7.5, k=2.5, \theta=45^{\circ} \ldots \ldots \ldots 136$

C.17 Normalized influence function coefficients, $K_{I}^{(i) *}=$ $K_{I}^{(i)} /\left[A_{i}(b / W)^{i} \sqrt{\pi b}\right]$, at nodal points along a semi-elliptical crack-front as defined by $\phi$ for $R^{*}=7.5, k=2.5, \theta=67.5^{\circ} \ldots \ldots \ldots 137$

C.18 Normalized influence function coefficients, $K_{I}^{(i) *}=$ $K_{I}^{(i)} /\left[A_{i}(b / W)^{i} \sqrt{\pi b}\right]$, at nodal points along a semi-elliptical crack-front as defined by $\phi$ for $R^{*}=7.5, k=2.5, \theta=90^{\circ} \ldots \ldots \ldots 138$

C.19 Normalized influence function coefficients, $K_{I}^{(i) *}=$ $K_{I}^{(i)} /\left[A_{i}(b / I V)^{i} \sqrt{\pi b}\right]$, at nodal points along a semi-elliptical crack-front as defined by $\phi$ for $R^{*}=10, k=1.5, \theta=45^{\circ} \ldots \ldots \ldots$ 
C.20 Normalized influence function coefficients, $K_{I}^{(i) *}=$ $K_{I}^{(i)} /\left[A_{i}(b / W)^{i} \sqrt{\pi b}\right]$, at nodal points along a semi-elliptical crack-front as defined by $\phi$ for $R^{*}=10, k=1.5, \theta=67.5^{\circ} \ldots \ldots \ldots$

C.21 Normalized influence function coefficients, $K_{I}^{(i) *}=$ $K_{I}^{(i)} /\left[A_{i}(b / W)^{i} \sqrt{\pi b}\right]$, at nodal points along a semi-elliptical crack-front as defined by $\phi$ for $R^{*}=10, k=1.5, \theta=90^{\circ} \ldots \ldots \ldots 141$

C.22 Normalized influence function coefficients, $K_{I}^{(i) *}=$ $K_{I}^{(i)} /\left[\Lambda_{i}(b / W)^{i} \sqrt{\pi b}\right]$, at nodal points along a semi-elliptical crack-front as defined by $\phi$ for $R^{*}=10, k=2, \theta=45^{\circ} \ldots \ldots \ldots 142$

C.23 Normalized influence function coefficients, $K_{I}^{(i) *}=$ $K_{I}^{(i)} /\left[A_{i}(b / W)^{i} \sqrt{\pi b}\right]$, at nodal points along a semi-elliptical crack-front as defined by $\phi$ for $R^{*}=10, k=2, \theta=67.5^{\circ} \ldots \ldots \ldots 143$

C.24 Normalized influence function coefficients, $K_{I}^{(i) *}=$ $K_{I}^{(i)} /\left[A_{i}(b / W)^{i} \sqrt{\pi b}\right]$, at nodal points along a semi-elliptical crack-front as defined by $\phi$ for $R^{*}=10, k=2, \theta=90^{\circ} \ldots \ldots \ldots 144$

C.25 Normalized influence function coefficients, $K_{I}^{(i) *}=$ $K_{I}^{(i)} /\left[A_{i}(b / W)^{i} \sqrt{\pi b}\right]$, at nodal points along a semi-elliptical crack-front as defined by $\phi$ for $R^{*}=10, k=2.5, \theta=45^{\circ}$

C.26 Normalized influence function coefficients: $K_{I}^{(i) *}=$ $K_{I}^{(i)} /\left[A_{i}(b / W)^{i} \sqrt{\pi b}\right]$, at nodal points along a semi-elliptical crack-front as defined by $\phi$ for $R^{*}=10, k=2.5, \theta=67.5^{\circ} \ldots \ldots 146$

C.27 Normalized influence function coefficients, $K_{I}^{(i) *}=$ $K_{I}^{(i)} /\left[A_{i}(b / W)^{i} \sqrt{\pi b}\right]$, at nodal points along a semi-elliptical crack-front as defined by $\phi$ for $R^{*}=10, k=2.5, \theta=90^{\circ} \ldots \ldots \ldots 147$

C.28 Comparison of normalized stress intensity factors, $K_{I} /(P \sqrt{\pi b})$, obtained from the influence coefficient method and by direct BEM along crack periphery, $\phi$, for $R^{*}=5, k=1.5, \theta=45^{\circ}$ 
C.29 Comparison of normalized stress intensity factors, $K_{I} /(P \sqrt{\pi b})$, obtained from the influence coefficient method and by direct BEM along crack periphery, $\phi$, for $R^{*}=5, k=1.5, \theta=67.5^{\circ} \ldots \ldots \ldots$

C.30 Comparison of normalized stress intensity factors, $K_{I} /(P \sqrt{\pi b})$, obtained from the influence coefficient method and by direct BEM along crack periphery, $\phi$, for $R^{*}=5, k=1.5, \theta=90^{\circ} \ldots \ldots \ldots$

C.31 Comparison of normalized stress intensity factors, $K_{I} /(P \sqrt{\pi b})$, obtained from the influcnce coefficient method and by direct BEM along crack periphery, $\phi$, for $R^{*}=5, k=2, \theta=45^{\circ}$

C.32 Comparison of normalized stress intensity factors, $K_{I} /(P \sqrt{\pi b})$, obtained from the influence coefficient method and by direct BEM along crack periphery, $\phi$, for $R^{*}=5, k=2, \theta=67.5^{\circ} \ldots \ldots \ldots$

C.33 Comparison of normalized stress intensity factors, $K_{I} /(P \sqrt{\pi b})$, obtained from the influence coefficient method and by direct BEM along crack periphery, $\phi$, for $R^{*}=5, k=2, \theta=90^{\circ} \ldots \ldots \ldots$

C.34 Comparison of normalized stress intensity factors, $K_{I} /(P \sqrt{\pi b})$, obtained from the influence coefficient method and by direct BEM along crack periphery, $\phi$, for $R^{*}=5, k=2.5, \theta=45^{\circ} \ldots \ldots \ldots$

C.35 Comparison of normalized stress intensity factors, $K_{I} /(P \sqrt{\pi b})$, obtained from the influence coefficient method and by direct BEM along crack periphery, $\phi$, for $R^{*}=5, k=2.5, \theta=67.5^{\circ} \ldots \ldots \ldots$

C.36 Comparison of normalized stress intensity factors, $K_{I} /(P \sqrt{\pi b})$, obtained from the influence coefficient method and by direct BEM along crack periphery, $\phi$, for $R^{*}=5, k=2.5, \theta=90^{\circ} \ldots \ldots \ldots$

C.37 Comparison of normalized stress intensity factors, $K_{I} /(P \sqrt{\pi b})$, obtained from the influence coefficient method and by direct BEM along crack periphery, $\phi$, for $R^{*}=7.5, k=1.5, \theta=45^{\circ}$ 
C.38 Comparison of normalized stress intensity factors, $K_{I} /(P \sqrt{\pi b})$, obtained from the influence coefficient method and by direct BEM along crack periphery, $\phi$, for $R^{*}=7.5, k=1.5, \theta=67.5^{\circ}$

C.39 Comparison of normalized stress intensity factors, $K_{I} /(P \sqrt{\pi b})$, obtained from the influence coefficient method and by direct BEM along crack periphery, $\phi$, for $R^{*}=7.5, k=1.5, \theta=90^{\circ} \ldots \ldots \ldots$

C.40 Comparison of normalized stress intensity factors, $K_{I} /(P \sqrt{\pi b})$, obtained from the influence coefficient method and by direct BEM along crack periphery, $\phi$, for $R^{*}=7.5, k=2, \theta=45^{\circ} \ldots \ldots \ldots 1$

C.41 Comparison of normalized stress intensity factors, $K_{I} /(P \sqrt{\pi b})$, obtained from the influence coefficient method and by direct BEM along crack periphery, $\phi$, for $R^{*}=7.5, k=2, \theta=67.5^{\circ}$

C.42 Comparison of normalized stress intensity factors, $K_{I} /(P \sqrt{\pi b})$, obtained from the influence coefficient method and by direct BEM along crack periphery, $\phi$, for $R^{*}=7.5, k=2, \theta=90^{\circ} \ldots \ldots \ldots 163$

C.43 Comparison of normalized stress intensity factors, $K_{I} /(P \sqrt{\pi b})$, obtained from the influence coefficient method and by direct BEM along crack periphery, $\phi$, for $R^{*}=7.5, k=2.5, \theta=45^{\circ} \ldots \ldots \ldots$

C.44 Comparison of normalized stress intensity factors, $K_{I} /(P \sqrt{\pi b})$, obtained from the influence coefficient method and by direct BEM along crack periphery, $\phi$, for $R^{*}=7.5, k=2.5, \theta=67.5^{\circ}$

C.45 Comparison of normalized stress intensity factors, $K_{I} /(P \sqrt{\pi b})$, obtained from the influence coefficient method and by direct BEM along crack periphery, $\phi$, for $R^{*}=7.5, k=2.5, \theta=90^{\circ} \ldots \ldots 166$

C.46 Comparison of normalized stress intensity factors, $K_{I} /(P \sqrt{\pi b})$, obtained from the influence coefficient method and by direct BEM along crack periphery, $\phi$, for $R^{*}=10, k=1.5, \theta=45^{\circ} \ldots \ldots \ldots 167$ 
C.47 Comparison of normalized stress intensity factors, $K_{I} /(P \sqrt{\pi b})$, obtained from the influence coefficient method and by direct BEM along crack periphery, $\phi$, for $R^{*}=10, k=1.5, \theta=67.5^{\circ} \ldots \ldots \ldots$

C.48 Comparison of normalized stress intensity factors, $K_{I} /(P \sqrt{\pi b})$, obtained from the influence coefficient method and by direct BEM along crack periphery, $\phi$, for $R^{*}=10, k=1.5, \theta=90^{\circ} \ldots \ldots \ldots$

C.49 Comparison of normalized stress intensity factors, $K_{I} /(P \sqrt{\pi b})$, obtained from the influence coefficient method and by direct BEM along crack periphery, $\phi$, for $R^{*}=10, k=2, \theta=45^{\circ} \ldots \ldots \ldots$

C.50 Comparison of normalized stress intensity factors, $K_{I} /(P \sqrt{\pi b})$, obtained from the influence coefficient method and by direct BEM along crack periphery, $\phi$, for $R^{*}=10, k=2, \theta=67.5^{\circ} \ldots \ldots \ldots$

C.51 Comparison of normalized stress intensity factors, $K_{I} /(P \sqrt{\pi b})$, obtained from the influence coefficient method and by direct BEM along crack periphery, $\phi$, for $R^{*}=10, k=2, \theta=90^{\circ} \ldots \ldots 172$

C.52 Comparison of normalized stress intensity factors, $K_{I} /(P \sqrt{\pi b})$, obtained from the influence coefficient method and by direct BEM along crack periphery, $\phi$, for $R^{*}=10, k=2.5, \theta=45^{\circ} \ldots \ldots \ldots$

C.53 Comparison of normalized stress intensity factors, $K_{I} /(P \sqrt{\pi b})$, obtained from the influence coefficient method and by direct BEM along crack periphery, $\phi$, for $R^{*}=10, k=2.5, \theta=67.5^{\circ} \ldots \ldots \ldots \ldots$

C.54 Comparison of normalized stress intensity factors, $K_{I} /(P \sqrt{\pi b})$, obtained from the influence coefficient method and by direct BEM along crack periphery, $\phi$, for $R^{*}=10, k=2.5, \theta=90^{\circ} \ldots \ldots 175$

D.1 Normalized stress intensity factors, $\left(K_{I}\right)_{A} /\left[\sigma_{Y} \sqrt{\pi b}\right]$, from residual stress due to autofrettage to different levels of overstrain with $R^{*}=5$, $k=2, \theta=45^{\circ}, b / W=0.2$ 
D.2 Normalized stress intensity factors, $\left(K_{I}\right)_{A} /\left[\sigma_{Y} \sqrt{\pi b}\right]$, from residual stress due to autofrettage to different levels of overstrain with $R^{*}=5$, $k=2, \theta=45^{\circ}, b / W=0$

D.3 Normalized stress intensity factors, $\left(K_{I}\right)_{A} /\left[\sigma_{Y} \sqrt{\pi b}\right]$, from residual stress due to autofrettage to different levels of overstrain with $R^{*}=5$, $k=2, \theta=45^{\circ}, b / W=0.6 \ldots \ldots \ldots \ldots \ldots \ldots \ldots \ldots \ldots$

D.4 Normalized stress intensity factors, $\left(K_{I}\right)_{A} /\left[\sigma_{Y} \sqrt{\pi b}\right]$, from residual stress due to autofrettage to different levels of overstrain with $R^{*}=5$, $k=2, \theta=45^{\circ}, b / W=0.8$

D.5 Normalized stress intensity factors, $\left(K_{I}\right)_{A} /\left[\sigma_{Y} \sqrt{\pi b}\right]$, from residual stress due to autofrettage to different levels of overstrain with $R^{*}=5$, $k=2, \theta=67.5^{\circ}, b / W=0.2$

D.6 Normalized stress intensity factors, $\left(K_{I}\right)_{A} /\left[\sigma_{Y} \sqrt{\pi b}\right]$, from residual stress due to autofrettage to different levels of overstrain with $R^{*}=5$, $k=2, \theta=67.5^{\circ}, b / W=0.4$

D.7 Normalized stress intensity factors, $\left(K_{I}\right)_{A} /\left[\sigma_{Y} \sqrt{\pi b}\right]$, from residual stress due to autofrettage to different levels of overstrain with $R^{*}=5$, $k=2, \theta=67.5^{\circ}, b / W=0.6$

D.8 Normalized stress intensity factors, $\left(K_{I}\right)_{A} /\left[\sigma_{Y} \sqrt{\pi b}\right]$, from residual stress due to autofrettage to different levels of overstrain with $R^{*}=5$, $k=2, \theta=67.5^{\circ}, b / W=0.8 \ldots \ldots \ldots \ldots$

D.9 Normalized stress intensity factors, $\left(K_{I}\right)_{A} /\left[\sigma_{Y} \sqrt{\pi b}\right]$, from residual stress due to autofrettage to different levels of overstrain with $R^{*}=5$,

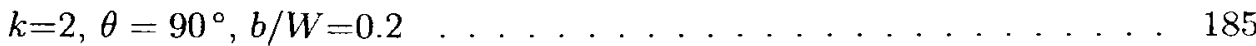

D.10 Normalized stress intensity factors, $\left(K_{I}\right)_{A} /\left[\sigma_{Y} \sqrt{\pi b}\right]$, from residual stress duc to autofrettage to different levels of overstrain with $R^{*}=5$, $k=2, \theta=90^{\circ}, b / W=0.4$. 
D.11 Normalized stress intensity factors, $\left(K_{I}\right)_{A} /\left[\sigma_{Y} \sqrt{\pi b}\right]$, from residual stress due to autofrettage to different levels of overstrain with $R^{*}=5$, $k=2, \theta=90^{\circ}, b / W=0 . \dot{6}$

D.12 Normalized stress intensity factors, $\left(K_{I}\right)_{A} /\left[\sigma_{Y} \sqrt{\pi b}\right]$, from residual stress due to autofrettage to different levels of overstrain with $R^{*}=5$, $k=2, \theta=90^{\circ}, b / W=0.8 \ldots \ldots \ldots \ldots$

D.13 Normalized stress intensity factors, $\left(K_{I}\right)_{A} /\left[\sigma_{Y} \sqrt{\pi b}\right]$, from residual stress due to autofrettage to different levels of overstrain with $R^{*}=7.5$, $k=2, \theta=45^{\circ}, b / W=0.2 \ldots \ldots \ldots \ldots$

D.14 Normalized stress intensity factors, $\left(K_{I}\right)_{A} /\left[\sigma_{Y} \sqrt{\pi b}\right]$, from residual stress due to autofrettage to different levels of overstrain with $R^{*}=7.5$, $k=2, \theta=45^{\circ}, b / W=0.4 \ldots \ldots \ldots \ldots \ldots$

D.15 Normalized stress intensity factors, $\left(K_{I}\right)_{A} /\left[\sigma_{Y} \sqrt{\pi b}\right]$, from residual stress due to autofrettage to different levels of overstrain with $R^{*}=7.5$, $k=2, \theta=45^{\circ}, b / W=0.6$

D.16 Normalized stress intensity factors, $\left(K_{I}\right)_{A} /\left[\sigma_{Y} \sqrt{\pi b}\right]$, from residual stress due to autofrettage to different levels of overstrain with $R^{*}=7.5$, $k=2, \theta=45^{\circ}, b / W=0.8$

D.17 Normalized stress intensity factors, $\left(K_{I}\right)_{A} /\left[\sigma_{Y} \sqrt{\pi b}\right]$, from residual stress due to autofrettage to different levels of overstrain with $R^{*}=7.5$, $k=2, \theta=67.5^{\circ}, b / W=0.2 \ldots \ldots \ldots$

D.18 Normalized stress intensity factors, $\left(K_{I}\right)_{A} /\left[\sigma_{Y} \sqrt{\pi b}\right]$, from residual stress due to autofrettage to different levels of overstrain with $R^{*}=7.5$, $k=2, \theta=67.5^{\circ}, b / W=0.4 \ldots \ldots \ldots \ldots$

D.19 Normalized stress intensity factors, $\left(K_{I}\right)_{A} /\left[\sigma_{Y} \sqrt{\pi b}\right]$, from residual stress duc to autofrcttage to different levels of overstrain with $R^{*}=7.5$, $k=2, \theta=67.5^{\circ}, b / W=0$. 
D.20 Normalized stress intensity factors, $\left(K_{I}\right)_{A} /\left[\sigma_{Y} \sqrt{\pi b}\right]$, from residual stress due to autofrettage to different levels of overstrain with $R^{*}=7.5$, $k=2, \theta=67.5^{\circ}, b / W=0.8$

D.21 Normalized stress intensity factors, $\left(K_{I}\right)_{A} /\left[\sigma_{Y} \sqrt{\pi b}\right]$, from residual stress due to autofrettage to different levels of overstrain with $R^{*}=7.5$, $k=2, \theta=90^{\circ}, b / W=0.2$

D.22 Normalized stress intensity factors, $\left(K_{I}\right)_{A} /\left[\sigma_{Y} \sqrt{\pi b}\right]$, from residual stress due to autofrettage to different levels of overstrain with $R^{*}=7.5$, $k=2, \theta=90^{\circ}, b / W=0.4$

D.23 Normalized stress intensity factors, $\left(K_{I}\right)_{A} /\left[\sigma_{Y} \sqrt{\pi b}\right]$, from residual stress due to autofrettage to different levels of overstrain with $R^{*}=7.5$, $k=2, \theta=90^{\circ}, b / W=0.6 \ldots \ldots \ldots \ldots$

D.24 Normalized stress intensity factors, $\left(K_{I}\right)_{A} /\left[\sigma_{Y} \sqrt{\pi b}\right]$, from residual stress due to autofrettage to different levels of overstrain with $R^{*}=7.5$, $k=2, \theta=90^{\circ}, b / W=0.8 \ldots \ldots \ldots \ldots$

D.25 Normalized stress intensity factors, $\left(K_{I}\right)_{A} /\left[\sigma_{Y} \sqrt{\pi b}\right]$, from residual stress due to autofrettage to different levels of overstrain with $R^{*}=10$,

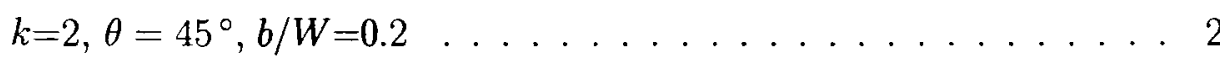

D.26 Normalized stress intensity factors, $\left(K_{I}\right)_{A} /\left[\sigma_{Y} \sqrt{\pi b}\right]$, from residual stress due to autofrettage to different levels of overstrain with $R^{*}=10$, $k=2, \theta=45^{\circ}, b / W=0.4 \ldots \ldots \ldots \ldots \ldots \ldots$

D.27 Normalized stress intensity factors, $\left(K_{1}\right)_{A} /\left[\sigma_{Y} \sqrt{\pi b}\right]$, from residual stress due to autofrettage to different levels of overstrain with $R^{*}=10$, $k=2, \theta=45^{\circ}, b / W=0.6 \ldots \ldots \ldots \ldots$

D.28 Normalized stress intensity factors, $\left(K_{I}\right)_{A} /\left[\sigma_{Y} \sqrt{\pi b}\right]$, from residual stress due to autofrettage to different levels of overstrain with $R^{*}=10$, $k=2, \theta=45^{\circ}, b / W=0.8$ 
D.29 Normalized stress intensity factors, $\left(K_{I}\right)_{A} /\left[\sigma_{Y} \sqrt{\pi b}\right]$, from residual stress due to autofrettage to different levels of overstrain with $R^{*}=10$, $k=2, \theta=67.5^{\circ}, b / W=0.2$

D.30 Normalized stress intensity factors, $\left(K_{I}\right)_{A} /\left[\sigma_{Y} \sqrt{\pi b}\right]$, from residual stress due to autofrettage to different levels of overstrain with $R^{*}=10$, $k=2, \theta=67.5^{\circ}, b / W=0.4 \ldots \ldots \ldots \ldots$

D.31 Normalized stress intensity factors, $\left(K_{I}\right)_{A} /\left[\sigma_{Y} \sqrt{\pi b}\right]$, from residual stress due to autofrettage to different levels of overstrain with $R^{*}=10$, $k=2, \theta=67.5^{\circ}, b / W=0$.

D.32 Normalized stress intensity factors, $\left(K_{I}\right)_{A} /\left[\sigma_{Y} \sqrt{\pi b}\right]$, from residual stress due to autofrettage to different levels of overstrain with $R^{*}=10$, $k=2, \theta=67.5^{\circ}, b / W=0.8$

D.33 Normalized stress intensity factors, $\left(K_{I}\right)_{A} /\left[\sigma_{Y} \sqrt{\pi b}\right]$, from residual stress due to autofrettage to different levels of overstrain with $R^{*}=10$, $k=2, \theta=90^{\circ}, b / \boldsymbol{W}=0.2 \ldots \ldots \ldots \ldots \ldots$

D.34 Normalized stress intensity factors, $\left(K_{I}\right)_{A} /\left[\sigma_{Y} \sqrt{\pi b}\right]$, from residual stress due to autofrettage to different levels of overstrain with $R^{*}=10$,

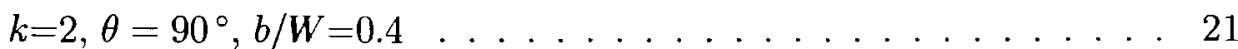

D.35 Normalized stress intensity factors, $\left(K_{I}\right)_{A} /\left[\sigma_{Y} \sqrt{\pi b}\right]$, from residual stress due to autofrettage to different levels of overstrain with $R^{*}=10$,

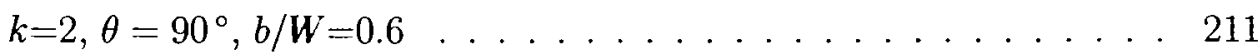

D.36 Normalized stress intensity factors, $\left(K_{I}\right)_{A} /\left[\sigma_{Y} \sqrt{\pi b}\right]$, from residual stress due to autofrettage to different levels of overstrain with $R^{*}=10$,

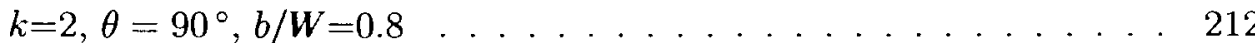




\section{List of Figures}

2.1 Boundary value problem in elastostatics . . . . . . . . . . . 28

2.2 Six node triangular element . . . . . . . . . . . . . . . . . 29

2.3 Eight node quadrilateral element . . . . . . . . . . . . 30

2.4 Circular hole in an "infinite" plate of finite thickness . . . . . . . . 31

2.5 Discretization of the problem of a circular hole in an "infinite" plate

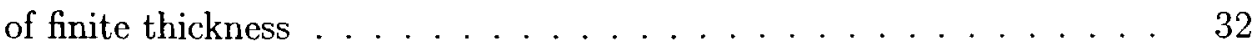

2.6 Variation of stress concentration factor, $\sigma_{y} / \sigma$, through plate thickness 33

2.7 Three displacement modes for crack surfaces $\ldots \ldots . . \ldots 34$

2.8 Crack-tip stresses shown with the Cartesian coordinate system . . 35

2.9 Crack-front element $\ldots \ldots \ldots \ldots \ldots$

2.10 BEM mesh for problem of a circular embedded crack in an "infinite"

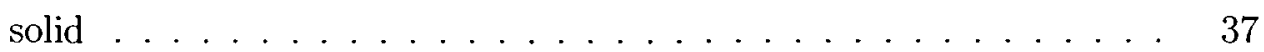

2.11 Semi-elliptical surface crack in a thick-walled cylinder f . . . . . 38

$2.12 \mathrm{BEM}$ mesh for cylinder with an elliptical crack, $k=2.5, b / W=0.4 \quad \ldots \quad 39$

2.13 Normalized stress intensity factors, $K_{I} /(P \sqrt{\pi b})$, for $k=2, b / W=0.4 \quad$. 40

2.14 Normalized stress intensity factors, $K_{I} /(P \sqrt{\pi b})$, for $k=2.5, b / W=0.2 \quad 41$

2.15 Normalized stress intensity factors, $K_{I} /(P \sqrt{\pi b})$, for $k=2.5, b / W=0.442$

2.16 Normalized stress intensity factors, $K_{I} /(P \sqrt{\pi b})$, for $k=2.5, b / W=0.6 \quad 43$

2.17 Principle of the weight function method . . . . . . . . . . . 44 
2.18 Normalized stress intensity factors, $K_{I} /(P \sqrt{\pi b})$, using influence coefficients for $k=2, b / W=0.4 \ldots \ldots \ldots \ldots \ldots$

2.19 Typical residual hoop stress distribution in an autofrettaged cylinder 46

2.20 Residual hoop stress, $\sigma_{\theta}$, through wall thickness of cylinder autofrettaged to $50 \%$ overstrain . . . . . . . . . . . . . 47

2.21 Normalized stress intensity factors, $K_{I} /\left(\sigma_{y} \sqrt{\pi b}\right)$, for $20 \%$ overstrain using influence coefficients for $k=2, b / W=0.4 \ldots \ldots . \ldots 48$

2.22 Normalized stress intensity factors, $K_{I} /\left(\sigma_{y} \sqrt{\pi b}\right)$, for $50 \%$ overstrain using influence coefficients for $k=2, b / W=0.4 \ldots \ldots$. . . . . . 49

3.1 Curved tubing diagram $\ldots \ldots \ldots \ldots \ldots$

3.2 Quarter-model mesh for $R^{*}=5, k=2, \theta=90^{\circ} \ldots \ldots \ldots 5$

3.3 Normalized hoop stress, $\sigma_{\psi} / \sigma_{o}$, at the inner radius $R_{1}$ at the intrados along the circumferential direction, $\theta$, of the pipe bend $R^{*}=5, k=1.5$,

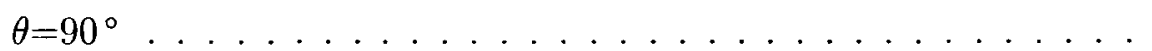

3.4 Normalized hoop stress, $\sigma_{\psi} / \sigma_{o}$, at the inner radius $R_{1}$ at the intrados along the circumferential direction, $\theta$, for $R^{*}=7.5, k=1.5, \theta=90^{\circ} \quad \ldots \quad 57$

3.5 Normalized hoop stress, $\sigma_{\psi} / \sigma_{o}$, for $R^{*}=5, k=2, \theta=90^{\circ}$ in circumferential direction, $\theta$, at intrados and extrados . . . . . . . . 58

3.6 Normalized hoop stress, $\sigma_{\psi} / \sigma_{o}$, at the intrados along the circumferential direction, $\theta$, for pipe bends $R^{*}=5, \theta=90^{\circ} \ldots \ldots \ldots$

3.7 Normalized hoop stress, $\sigma_{\psi} / \sigma_{o}$, at the intrados along the circumferential direction, $\theta$, for pipe bends $R^{*}=7.5, \theta=90^{\circ} \ldots \ldots \ldots$

3.8 Normalized hoop stress, $\sigma_{\psi} / \sigma_{o}$, at the intrados along the circumferential direction, $\theta$, for pipe bends $R^{*}=10, \theta=90^{\circ}$

3.9 Normalized hoop stress, $\sigma_{\psi} / \sigma_{o}$, at the intrados along the circumferential direction, $\theta$, for pipe bends $k=1.5, \theta=90^{\circ} \ldots \ldots 62$ 
3.10 Normalized hoop stress, $\sigma_{\psi} / \sigma_{o}$, at the intrados along the circumferential direction, $\theta$, for pipe bends $k=2, \theta=90^{\circ} \ldots \ldots 63$

3.11 Normalized hoop stress, $\sigma_{\psi} / \sigma_{o}$, at the intrados along the circumferential direction, $\theta$, for pipe bends $k=2.5, \theta=90^{\circ} \ldots \ldots 64$

3.12 Normalized hoop stress, $\sigma_{\psi} / \sigma_{o}$, at the intrados along the circumferential direction, $\theta$, for pipe bends $R^{*}=5, k=2 \ldots \ldots \ldots 6$

3.13 Normalized hoop stress, $\sigma_{\psi} / \sigma_{o}$, at the intrados along the circumferential direction, $\theta$, for pipe bends $R^{*}=7.5, k=2 \ldots \ldots 66$

3.14 Normalized hoop stress, $\sigma_{\psi} / \sigma_{o}$, at the intrados along the circumferential direction, $\theta$, for pipe bends $R^{*}=10, k=2 \ldots \ldots 67$

4.1 Semi-elliptical crack in curved tubing . . . . . . . . . . . . . 91

4.2 Sample BEM mesh for $R^{*}=7.5, k=2.5, \theta=67.5^{\circ}, b / W=0.4$ with 112 elements and 332 nodes . . . . . . . . . . . . . . . 92

4.3 BEM discretization for mesh refinement study . . . . . . . . . . 93

4.4 Variation of the normalized stress intensity factor, $K_{I} /(P \sqrt{\pi b})$, along the crack-front, $\phi$, of pressurized curved tubing, $R^{*}=5, k=1.5$,

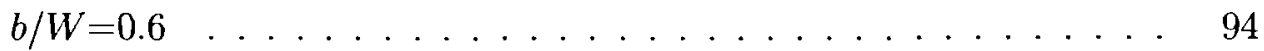

4.5 Variation of the normalized stress intensity factor, $K_{I} /(P \sqrt{\pi b})$, along the crack-front, $\phi$, of pressurized curved tubing, $R^{*}=5, k=2, b / W=0.6$

4.6 Variation of the normalized stress intensity factor, $K_{I} /(P \sqrt{\pi b})$, along the crack-front, $\phi$, of pressurized curved tubing, $R^{*}=5, k=2.5$,

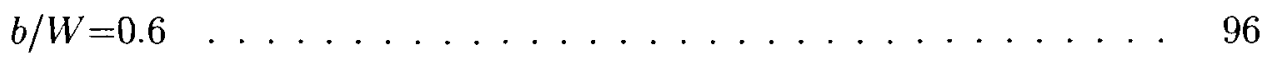

4.7 Variation of the normalized stress intensity factor, $K_{I} /(P \sqrt{\pi b})$, along the crack-front, $\phi$, of pressurized curved tubing, $R^{*}=7.5, k=2.5$, $b / W=0.6 \ldots \ldots \ldots \ldots \ldots \ldots \ldots \ldots$ 
4.8 Variation of the normalized stress intensity factor, $K_{I} /(P \sqrt{\pi b})$, along the crack-front, $\phi$, of pressurized curved tubing, $R^{*}=10, k=2.5$,

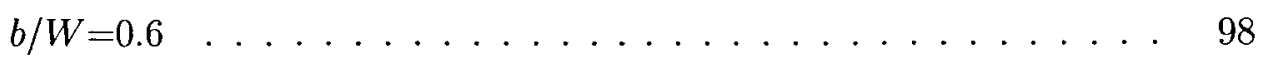

4.9 Variation of the normalized stress intensity factor, $K_{I} /(P \sqrt{\pi b})$, along the crack-front, $\phi$, of pressurized curved tubing, $R^{*}=5, k=2, \theta=90^{\circ} \quad . \quad 99$ 


\title{
Nomenclature
}

\author{
Arabic Letters \\ $A_{i} \quad$ polynomial coefficients \\ $E \quad$ Young's modulus \\ $G \quad$ Shear modulus \\ $J \quad$ Jacobian of transformation \\ $K_{I} \quad$ mode I stress intensity factor \\ $K_{l}^{(i)} \quad$ polynomial influence coefficients \\ $N_{d}^{i}, N_{t}^{i} \quad$ displacement and traction shape function respectively at $i$ th node \\ $P \quad$ uniform hydrostatic pressure \\ $Q \quad$ surface field point \\ $R \quad$ radius of curvature \\ $R_{1} \quad$ inner radius \\ $R_{2} \quad$ outer radius \\ $R^{*} \quad R / R_{1}$ ratio \\ $W \quad$ wall thickness \\ semi-major elliptical axis \\ half-crack length \\ $b \quad$ semi-minor elliptical axis \\ $k \quad R_{2} / R_{1}$ ratio \\ $l \quad$ length of crack tip element \\ $n_{j} \quad$ unit outward normal vector \\ $n R_{1} \quad$ depth of plastic region \\ $p \quad$ load point \\ $q \quad$ field point \\ distance from load point to field point \\ $r \quad$ cylindrical and toroidal coordinate radial direction \\ $r_{s} \quad$ distance through thickness from internal radius
}




$\begin{array}{ll}t_{j} & \text { tractions } \\ u_{j} & \text { displacements }\end{array}$

$\begin{array}{cl}\text { Greek Letters } & \\ \Gamma & \text { boundary of a body } \\ \Omega & \text { domain of a body } \\ \delta_{i j} & \text { Kronecker delta } \\ \epsilon_{i j} & \text { strain tensor } \\ \theta & \text { toroidal coordinate longitudinal angle } \\ \kappa & \text { cylindrical coordinate circumferential angle } \\ \nu & \text { plane stress or plane strain } \\ \xi_{i} & \text { intrinsic local coordinate } \\ \sigma & \text { applied stress } \\ \sigma_{y} & \text { yield stress } \\ \sigma_{i j} & \text { stress tensor } \\ \phi & \text { angle of crack profile } \\ \psi & \text { toroidal coordinate circumferential angle }\end{array}$




\section{Chapter 1}

\section{Introduction}

With advancing technology, pipelines and piping components are carrying higher pressure loads, and thus thick-walled tubing is increasingly being used. The most susceptible point of any pipeline is within curved sections and therefore the stress distribution throughout this area is investigated. Due to the vulnerability of these components to cracking, the stress intensity factors for a semi-elliptical crack are determined for a range of parameters. To increase the versatility of the stress intensity factor results, a full list of polynomial influence coefficients is also presented. These may be applied to any load case with the same cracked geometry and this is demonstrated with an analysis of partially autofrettaged curved tubing. Autofrettage is a technique used to extend the fatigue life of process piping and weaponry by imparting beneficial residual compressive stresses.

\subsection{Previous Work}

A number of analyses have been conducted on semi-elliptical cracks in thick-walled internally pressurized cylinders. Varying in crack geometry or other parameters investigated, this has been studied by Tan \& Fenner (1980), Nadiri, Tan \& Fenner (1982), and Shim (1986) by employing the boundary element method (BEM), as well 
as Blackburn \& Hellen (1977), Atluri \& Kathiresan (1979), and Pu \& Hussain (1981) using the finite element method (FEM). This has been limited to straight circular cylinders however.

Early experimental work on stresses in thick-walled pipe bends was performed by Swanson \& Ford (1959) under in-plane bending and internal pressure. This study compared the experimental results from cold-rolled pipe bends with previously published theoretical analyses by von Kármán (1911), Barthélemy (1947), Thuloup (1937), and Turner \& Ford (1957), none of which applied specifically to thick-walled pressurized pipe bends, however.

Further analysis of stress distributions in pressurized elbows and pipe bends was presented by Lang (1984), and introduced as Toroidal Elasticity theory. The approximate solutions obtained were in the form of series. This was applied to a torus by Colle, Redekop \& Tan (1987) who also employed the BEM in three-dimensions to verify Lang's series solution. Toroidal Elasticity theory and its use for pressurized elbows was limited to uncracked geometries under various loading conditions, and the BEM work of Colle et al. (1987) on a torus was also restricted to the stress concentration problem of a cross-bore without any cracks present.

The autofrettage of pipe bends was analyzed by Rees (2004) but was similarly limited to uncracked geometries; this work entailed bending of a pre-autofrettaged tube and investigated the stresses created by this process. The current work assumes a pipe bend manufactured as is, with no pre-stresses present, nor cross-sectional nonuniformities such as localized wall thinning. It should be noted that when subjected to internal pressure, these defects, particularly local flattening, can result in large stresses. 


\subsection{Proposed Solution Method}

Due to the complexity of the geometry, no exact theoretical solution for the physical problem of a thick-walled pressurized curved tubing is available; this is particularly so when a crack is present. Recourse to numerical methods is commonly adopted in such circumstances and an established computational technique for stress analysis is the boundary element method.

The boundary element method (BEM) was selected as the solution method for the physical problem due to its efficiency with problems with high stress gradients present such as those near stress concentrations and cracks. The BEM differs from the well-known finite element method (FEM) in that for a three-dimensional component, only the surface needs be discretized into elements as opposed to the entire volume; if required, interior points may be solved for separately as a secondary exercise. The treatment of only the surface instead of the volume of the problem effectively reduces the numerical dimension of the problem by one. Thus, the number of final algebraic equations to be solved may commonly be reduced by at least one order of magnitude, thereby reducing data preparation and solution times, particularly for complicated geometries.

\subsection{Outline}

A brief introduction to the BEM is presented in the next chapter. In addition the theory of linear elastic fracture mechanics and the weight function method, particularly polynomial influence coefficients, for use with determining stress intensity factors for cracked components will also be reviewed. These influence coefficients may be applied for any load case with the same crack geometry. A cylinder which has been subjected to partial autofrettage is introduced to demonstrate this concept. 
The stress distribution in an uncracked, internally pressurized thick-walled pipe bend is studied in Chapter 3. The location of the peak stress in such a component is identified; it is where a crack would most likely develop in the physical problem. To this end, stress factors are determined for a range of a number of parameters, namely bend radius, radius ratio of the cross-section, and angle of curvature.

Once the most likely site for crack nucleation has been determined, in Chapter 4, a semi-elliptical crack is introduced for the fracture mechanics analysis into all the previous uncracked models mentioned above. Only one crack shape, namely, that corresponding to a semi-minor to semi-major axis ratio, $b / a$, of 0.8 is chosen for this study. The primary reason for this is that it would allow direct comparisons with similar results which are available in the literature for a straight tubing as this crack shape is very commonly found in thick-walled cylinders of radius ratios between 2 and 3. Stress intensity factors are determined along the crack periphery for each case. To lend more versatility to the results, polynomial influence coefficients are also determined for each instance. They are used to verify the stress intensity factors computed directly from the BEM. Finally, the application of these influence coefficients is demonstrated by determining the stress intensity factors due to the residual stresses in an autofrettaged tubing which has been subjected to various percentages of overstrain in a representative set of the numerical models. 


\section{Chapter 2}

\section{Review of the Boundary Element Method}

The boundary element method (BEM) is a powerful numerical tool for solving elastostatic problems, particularly those with high stress gradients. Its analytical and numerical formulation are widely published in the literature (see, e.g. Becker (1992)) and therefore only a brief review is presented here. The BEM is particularly efficient for obtaining accurate solutions to problems with stress concentrations; it can also be applied to linear elastic fracture mechanics analysis for obtaining stress intensity factors with minimal modifications.

\subsection{Formulation of the BEM in Three-Dimensions}

Using Einstein notation where repeated indices denote summation and a comma index is used to represent the derivative, the stresses in a linear elastic solid domain, $\Omega$, in static equilibrium are governed by

$$
\sigma_{i j, j}+f_{i}=0 \text { in } \mathrm{R} ; \quad \mathrm{i}, \mathrm{j}=1,2,3 \text { in three-dimensions }
$$


where $\sigma_{i j}$ is the second order stress tensor and $\int_{i}$ are the components of the body force vector per unit volume. In addition, the strain-displacement relationship is given by

$$
\epsilon_{i j}=\frac{1}{2}\left(u_{i, j}+u_{j, i}\right)
$$

where $\epsilon_{i j}$ is the strain tensor and $u_{i}$ is the displacement component in the $x_{i}$ - direction. Furthermore, Hooke's law is defined as

$$
\sigma_{i j}=2 G\left(\epsilon_{i j}+\frac{\nu}{1-2 \nu} \epsilon_{k k} \delta_{i j}\right)
$$

where $\delta_{i j}$ is the Kronecker delta, $\nu$ is the Poisson's ratio and $G$ is the shear modulus.

Substituting Equation 2.3 into 2.1 with use of Equation 2.2 yields the CauchyNavier equation, which in the absence of body forces, becomes

$$
G\left(u_{i, j j}+\frac{1}{1-2 \nu} u_{j, i i}\right)=0
$$

Solving Equation 2.4 for a region $\Omega$ bounded by $\Gamma$ necessitates the following boundary conditions.

$$
\begin{aligned}
& u_{i}(x)=g_{i}(x) \quad \text { on } \Gamma_{u} \\
& t_{i}(x)=\sigma_{i j} n_{j}=h_{i}(x) \quad \text { on } \Gamma_{t}
\end{aligned}
$$

where $u_{i}(x)$ and $t_{i}(x)$ are the displacement and traction components, respectively, with $g_{i}(x)$ and $h_{i}(x)$ as prescribed functions on the boundary $\Gamma$. The boundary can be represented by the summation

$$
\Gamma \epsilon\left(\Gamma_{u}+\Gamma_{t}\right)
$$


and $n_{j}$ is the unit outward normal vector on $\Gamma$.

The fundamental solution to Equation 2.4 is given by Kelvin's unit point load solution for an infinite body. The displacement and traction tensors for this problem in three-dimensions are given by Cruse (1969) as

$$
\begin{aligned}
U_{i j}(p, q) & =\frac{1}{16 \pi G(1-\nu) r}\left[(3-4 \nu) \delta_{i j}+r_{, i} r_{, j}\right] \\
T_{i j}(p, q) & =\frac{-(1-2 \nu)}{8 \pi(1-\nu) r^{2}}\left\{\frac{\partial r}{\partial n}\left[\delta_{i j}+\frac{3}{1-2 \nu} r_{, i} r_{, j}\right]-r_{, i} n_{j}+r_{, j} n_{i}\right\}
\end{aligned}
$$

Here, $r$ is the distance from the load point $p$ to the field point $q$ (or surface field point $Q)$ as shown in Figure 2.1 and is defined below.

$$
\begin{aligned}
r & =\sqrt{\left(x_{Q_{i}}-x_{p_{i}}\right)\left(x_{Q_{i}}-x_{p_{i}}\right)} \\
r_{, i} & =\frac{\partial r}{\partial x_{Q_{i}}}=\frac{\left(x_{Q_{i}}-x_{p_{i}}\right)}{r}
\end{aligned}
$$

Substituting the fundamental solutions into Betti's reciprocal work theorem, see, e.g. Becker (1992), and employing the divergence theorem yields Somigliana's identity:

$$
u_{i}(p)=\int_{\Gamma} t_{i}(Q) U_{i j}(p, Q) d \Gamma-\int_{\Gamma} u_{j}(Q) T_{i j}(p, Q) d \Gamma
$$

From this relation, the displacements at an internal point may be obtained in terms of the boundary data. Moving the interior point to the boundary through the usual limiting process results in the boundary integral equation which relates boundary tractions to boundary displacements. Neglecting the influence of body forces, the boundary integral equation can be written as

$$
C_{i j}(P) u_{j}(P)+\int_{\Gamma} T_{i j}(P, Q) u_{j}(Q) d \Gamma(Q)=\int_{\Gamma} U_{i j}(P, Q) t_{j}(Q) d \Gamma(Q)
$$


where

$$
C_{i j}(P)=\lim _{\epsilon \rightarrow 0} \int_{\Gamma_{\epsilon}} T_{i j}(P, Q) d \Gamma
$$

If $P$ lies on a smooth surface,

$$
C_{i j}(P)=\frac{1}{2} \delta_{i j}
$$

Stresses inside the domain, in the absence of body forces, can be determined from the corresponding Somigliana's stress identity wich can be derived using Equation 2.11, the strain-displacement relation and Hooke's law. This stress identity is given as follows:

$$
\sigma_{i j}(p)=\int_{\Gamma} D_{k i j}(p, Q) t_{k}(Q) d \Gamma(Q)-\int_{\Gamma} S_{k i j}(p, Q) u_{k}(Q) d \Gamma(Q)
$$

where

$$
\begin{aligned}
D_{k i j} & =\frac{1}{8 \pi(1-\nu) r^{2}}\left[3 r_{, k} r_{, i} r_{, j}+(1-2 \nu)\left(\delta_{k i} r_{, j}+\delta_{k j} r_{, i}-\delta_{i j} r, k\right)\right] \\
S_{k i j} & =\frac{G}{4 \pi(1-\nu) r^{3}}\left\{3 \frac{\partial r}{\partial n}\left[(1-2 \nu) \delta_{i j} r_{, k}+\nu\left(\delta_{k i} r_{, j}+\delta_{k j} r_{, i}\right)-5 r_{, k} r_{, i} r_{, j}\right]\right. \\
& +3 \nu\left(n_{i} r_{, k} r_{, j}+n_{j} r_{, i} r_{, k}\right)+(1-2 \nu)\left(3 n_{k} r_{, i} r_{, j}+n_{j} \delta_{k i}+n_{i} \delta_{k j}\right) \\
& \left.-(1-4 \nu) n_{k} \delta_{i j}\right\}
\end{aligned}
$$

\subsubsection{Numerical Treatment of the BEM in Three-Dimensions}

The numerical solution of the boundary integral equation requires the boundary, $\Gamma$, to be discretized into elements. Suitable algebraic functions are applied to the elements with requisite values at specified nodal points. From experience with the finitc element method, it has been determined that the best trade-off of efficiency for accuracy was with the use of quadratic isoparametric elements. The same has been 
found to be true for BEM. Thus, the surface of a three-dimensional component is represented with 6-node triangular and/or 8-node quadrilateral elements, as shown in Figures 2.2 and 2.3, respectively. Also similar to the finite element method, the interpolation functions applied to the elements to define the unknowns and geometry are quadratic shape functions in terms of the local intrinsic coordinates $\boldsymbol{\xi} \equiv \boldsymbol{\xi}_{j}, j=1,2$, as follows, from Zienkiewicz (1977) and Reddy (2006)

$$
\begin{aligned}
x_{i}(\boldsymbol{\xi}) & =N^{c}(\boldsymbol{\xi}) x_{i}^{c} \\
u_{i}(\boldsymbol{\xi}) & =N^{c}(\boldsymbol{\xi}) u_{i}^{c} \\
t_{i}(\boldsymbol{\xi}) & =N^{c}(\boldsymbol{\xi}) t_{i}^{c}
\end{aligned}
$$

where

$$
c=\left\{\begin{array}{cc}
1,2,3, \ldots, 8 \text { for quadrilateral elements } \\
1,2,3, \ldots, 6 \text { for triangular elements }
\end{array}\right.
$$

and $N^{c}(\xi)$ are the shape functions which are given explicitly in Appendix A.

If the surface is represented by $m$ surface elements and $q$ distinct nodes, then Equation 2.12 becomes

$$
\begin{aligned}
C_{i j}\left(P^{a}\right) u_{j}\left(P^{a}\right) & +\sum_{b=1}^{m} \sum_{c=1}^{k} u_{j}\left(P^{d(b, c)}\right) \int_{\Gamma} T_{i j}\left(P^{a}, Q(\boldsymbol{\xi})\right) N^{c}(\boldsymbol{\xi}) J(\boldsymbol{\xi}) d \boldsymbol{\xi} \\
& =\sum_{b=1}^{m} \sum_{c=1}^{k} t_{j}\left(P^{d(b, c)}\right) \int_{\Gamma} U_{i j}\left(P^{a}, Q(\boldsymbol{\xi})\right) N^{c}(\boldsymbol{\xi}) J(\boldsymbol{\xi}) d \boldsymbol{\xi}
\end{aligned}
$$

where $P^{a}$ is the ath node $(a=1, q), d(b, c)$ is the number of the $c$ th node of the $b$ th element, $k=6$ for a triangular element and 8 for a quadrilateral, and $J(\xi)$ is the Jacobian of transformation. Equation 2.19 represents a system of $3 q$ distinct linear equations to be solved to determine the unknown boundary tractions or displacements 
at the nodes. Once a solution for Equation 2.19 has been obtained, interior point solutions may be determined by substituting the isoparametric representations into Equations 2.11 and 2.15 .

\subsubsection{Example Problem - Stress Concentrations}

A typical stress concentration problem, that of a circular hole in an infinite plate of finitc thickness, was analyzed to demonstrate the efficiency of the BEM. This threedimensional problem was selected to show the accuracy of the BEM in capturing stress concentrations with a relatively coarse mesh. The analytical solution to the problcm has been given by Sternberg \& Sadowsky (1949) and was verified with the BEM by Tan \& Fenner (1978).

As shown in Figure 2.4, the plate was defined with a height, $H$, equal to its width, $W$. In addition the thickness, $2 c$, was nominally set to $0.2 H$ and the radius of the cylindrical hole $a=0.1 H$. Poisson's ratio of 0.3 was assumed and a tensile stress of $\sigma$ was applied as indicated.

The physical problem was represented using a one-eighth model with 64 quadratic elements and 194 nodes. The periphery of the circular hole was modelled with four elements over $90^{\circ}$. It was constrained from displacement along the horizontal and vertical planes of symmetry, the $\mathrm{x}$ - and $\mathrm{y}$ - axes respectively as shown in Figure 2.5(a). Also, only one-half of the plate thickness was modelled and thus the lower surface was constrained from displacement in the $\mathrm{z}$ - direction. The problem was also analyzed with a commercial finite element method code, ABAQUS, and this model was discretized with 760 20-node brick elements, as shown in Figure 2.5(b) for comparison.

The results of both the present BEM and FEM studies, as well as the BEM results by Tan \& Fenner (1978) and the analytical solution by Sternberg \& Sadowsky (1949), are shown in Table 2.1 at the mid-thickness plane and at the free surface at the edge of the hole. It is evident that the present results are in good agreement 
with those determined previously for this problem. As no explicit numerical results are published at other points through the plate thickness by Tan \& Fenner (1978), a direct comparison cannot be made elsewhere; Figure 2.6 shows the variation of the stress concentration factor through the thickness of the plate for the present study as well as the analytical solution. The percentage discrepancy between the present BEM results and the analytical solution was less than $1.5 \%$ at the mid-thickness plane and $3.6 \%$ at the free surface, in spite of the relatively coarse mesh used.

\section{$2.2 \quad$ Fracture Problems}

In linear elastic fracture mechanics, the stress field near a crack-front may be characterized in term of the three modes of deformation. These are shown in Figure 2.7: mode I, the opening mode; mode II, the edge-sliding mode; and mode III, the shear or tearing mode. Although a fracture problem may consist of any combination of these three modes, the opening mode is considered to be the most important as it is the most common in practice. Therefore, throughout this study, only mode I deformation was considered.

Using linear elastic theory and the Westergaard stress function as shown in Unger (1995), the stress distribution near the crack-front under mode I conditions can be expressed as

$$
\left[\begin{array}{c}
\sigma_{x x} \\
\sigma_{y y} \\
\sigma_{x y}
\end{array}\right]=\frac{K_{I} \cos \left(\frac{\theta}{2}\right)}{\sqrt{2 \pi r}}\left[\begin{array}{c}
1-\sin \left(\frac{\theta}{2}\right) \sin \left(\frac{3 \theta}{2}\right) \\
1+\sin \left(\frac{\theta}{2}\right) \sin \left(\frac{3 \theta}{2}\right) \\
\sin \left(\frac{\theta}{2}\right) \cos \left(\frac{3 \theta}{2}\right)
\end{array}\right]+O\left(r^{o}\right)
$$


and

$$
\begin{aligned}
\sigma_{z z} & =\nu^{\prime}\left(\sigma_{x x}+\sigma_{y y}\right) \\
\sigma_{x z} & =\sigma_{y z}=0
\end{aligned}
$$

where $K_{l}$ is the mode I stress intensity factor, $r$ and $\theta$ are polar coordinates as indicated in Figure 2.8, $O\left(r^{\circ}\right)$ represents higher order terms that are not significant to the crack tip solution and $\nu$ is Poisson's ratio as follows

$$
\nu^{\prime}=\left\{\begin{array}{cc}
0 & \text { for plane stress } \\
\nu & \text { for plane strain }
\end{array}\right.
$$

The corresponding displacement field for the opening mode deformation is given as

$$
\begin{aligned}
{\left[\begin{array}{l}
u_{x} \\
u_{y}
\end{array}\right] } & =\frac{K_{I} \sqrt{\frac{r}{2 \pi}}(1+\nu)}{2 E}\left[\begin{array}{c}
(2 \kappa-1) \cos \left(\frac{\theta}{2}\right)-\cos \left(\frac{3 \theta}{2}\right) \\
(2 \kappa+1) \sin \left(\frac{\theta}{2}\right)-\sin \left(\frac{3 \theta}{2}\right)
\end{array}\right]+O\left(r^{\circ}\right) \\
u_{z} & =-\left(\nu^{\prime \prime} z / E\right)\left(\sigma_{x x}+\sigma_{y y}\right)
\end{aligned}
$$

where $\mathrm{E}$ is the Young's modulus, and

$$
\begin{array}{ll}
\text { for plane stress: } \nu^{\prime \prime}=\nu, \quad \kappa=\frac{(3-\nu)}{(1+\nu)} \\
\text { for plane strain: } \quad \nu^{\prime \prime}=0, \quad \kappa=(3-4 \nu)
\end{array}
$$

From Equation 2.20 and 2.24, it can be seen that the displacements vary accoding to $\sqrt{r}$ while the stresses near the crack-front are singular and vary according to $1 / \sqrt{r}$. 
The stress intensity factor, $K_{I}$, defines the magnitude of the stress singularity and is a function of geometry, crack size and the applied load.

\subsubsection{Crack-Front Boundary Elements}

In BEM, as in FEM modelling of crack problems, a commonly adopted means of introducing the $\sqrt{r}$ variation for the displacement field near the crack-front without any modification of the quadratic shape functions is to shift the mid-side node adjacent to the crack-front to the quarter-point position closest to the crack-front. Unlike in FEM, however, the associated $1 / \sqrt{r}$ traction singularity can be obtained by multiplying the shape functions for the crack-front element by $l / \sqrt{r}$ (see, e.g. Aliabadi \& Rooke (1991)).

In the BEM code employed in this study, BIE3D5, the crack-front quadrilateral elements are geometrically akin to isoparametric quadrilateral elements but with shape functions different from those in Section 2.1.1. As determined by Luchi \& Poggialini (1983), these shape functions for the quadrilateral elements provide the appropriate

displacement and traction variations near the crack-front. The following are the shape functions when nodes 1-2-3 lie along the crack-front, as shown in Figure 2.3, those 
corresponding to all sides of the element are listed in Appendix A.

$$
\begin{aligned}
& N_{d}^{1}\left(\xi_{1}, \xi_{2}\right)=\frac{1}{2}\left(1-\xi_{1}\right)\left[-\xi_{1}-\left(1-\frac{\sqrt{2}}{2} \xi_{1}\right) \sqrt{1+\xi 2}+\frac{\sqrt{2}}{2}\left(1+\xi_{2}\right)\right] \\
& N_{d}^{2}\left(\xi_{1}, \xi_{2}\right)=\left(1-\xi_{1}^{2}\right)\left[1-\frac{\sqrt{2}}{2} \sqrt{1+\xi_{2}}\right] \\
& N_{d}^{3}\left(\xi_{1}, \xi_{2}\right)=\frac{1}{2}\left(1+\xi_{1}\right)\left[\xi_{1}-\left(1+\frac{\sqrt{2}}{2} \xi_{1}\right) \sqrt{1+\xi 2}+\frac{\sqrt{2}}{2}\left(1+\xi_{2}\right)\right] \\
& N_{d}^{4}\left(\xi_{1}, \xi_{2}\right)=\frac{1}{2}\left(1+\xi_{1}\right)\left[(2+\sqrt{2}) \sqrt{1+\xi_{2}}-(1+\sqrt{2})\left(1+\xi_{2}\right)\right] \\
& N_{d}^{5}\left(\xi_{1}, \xi_{2}\right)=\frac{1}{4}\left(1+\xi_{1}\right)\left[\sqrt{2}\left(\xi_{1}-2-\sqrt{2}\right) \sqrt{1+\xi_{2}}+(2+\sqrt{2})\left(1+\xi_{2}\right)\right] \\
& N_{d}^{6}\left(\xi_{1}, \xi_{2}\right)=\frac{\sqrt{2}}{2}\left(1-\xi_{1}^{2}\right) \sqrt{1+\xi_{2}} \\
& N_{d}^{7}\left(\xi_{1}, \xi_{2}\right)=\frac{1}{4}\left(1-\xi_{1}\right)\left[-\sqrt{2}\left(\xi_{1}+2+\sqrt{2}\right) \sqrt{1+\xi_{2}}+(2+\sqrt{2})\left(1+\xi_{2}\right)\right] \\
& N_{d}^{8}\left(\xi_{1}, \xi_{2}\right)=\frac{1}{2}\left(1-\xi_{1}\right)\left[(2+\sqrt{2}) \sqrt{1+\xi_{2}}-(1+\sqrt{2})\left(1+\xi_{2}\right)\right]
\end{aligned}
$$

where $N_{d}^{i}$ denotes the shape function for the displacements of the crack-front element for the ith node. Similarly,

$$
\begin{aligned}
& N_{t}^{1}\left(\xi_{1}, \xi_{2}\right)=N_{d}^{1}\left(\xi_{1}, \xi_{2}\right) / \sqrt{1+\xi_{2}} \\
& N_{t}^{2}\left(\xi_{1}, \xi_{2}\right)=N_{d}^{2}\left(\xi_{1}, \xi_{2}\right) / \sqrt{1+\xi_{2}} \\
& N_{t}^{3}\left(\xi_{1}, \xi_{2}\right)=N_{d}^{3}\left(\xi_{1}, \xi_{2}\right) / \sqrt{1+\xi_{2}} \\
& N_{t}^{4}\left(\xi_{1}, \xi_{2}\right)=N_{d}^{4}\left(\xi_{1}, \xi_{2}\right) / \sqrt{1+\xi_{2}} \\
& N_{t}^{5}\left(\xi_{1}, \xi_{2}\right)=\sqrt{2} N_{d}^{5}\left(\xi_{1}, \xi_{2}\right) / \sqrt{1+\xi_{2}} \\
& N_{t}^{6}\left(\xi_{1}, \xi_{2}\right)=\sqrt{2} N_{d}^{6}\left(\xi_{1}, \xi_{2}\right) / \sqrt{1+\xi_{2}} \\
& N_{t}^{7}\left(\xi_{1}, \xi_{2}\right)=\sqrt{2} N_{d}^{7}\left(\xi_{1}, \xi_{2}\right) / \sqrt{1+\xi_{2}} \\
& N_{t}^{8}\left(\xi_{1}, \xi_{2}\right)=N_{d}^{8}\left(\xi_{1}, \xi_{2}\right) / \sqrt{1+\xi_{2}}
\end{aligned}
$$

where $N_{t}^{i}$ denotes the shape function for the tractions on the crack-front elements for the $i$ th node. 
By equating the variations of the displacements and tractions along the crackfront element, as represented by the shape functions described above, to the classical field solution in Section 2.2 simple expressions for the direct evalution of the stress intensity factors from the computer BEM model results may be obtained. These have been derived by Luchi \& Poggialini (1983) for three-dimensions.

Mode I stress intensity factors can be obtained using the computed traction data (the "traction formula"), as follows,

$$
K_{I}^{A}=t_{1}^{A} \sqrt{\pi l}
$$

where the superscript refers to the node indicated in Figure 2.9 along the crack face, and $l$ is the length of the crack tip element corrected to be perpendicular to the crackfront. Similarly, when using the displacement data, the "displacement formulae" may be used for the direct evaluation of the stress intensity factor:

$$
\begin{aligned}
K_{I}^{A} & =\frac{2 G}{\kappa+1} \sqrt{\frac{\pi}{l}}\left(u_{1}^{D}-u_{1}^{I}\right) \\
K_{I}^{B} & =\frac{2 G}{\kappa+1} \sqrt{\frac{\pi}{2 l}}\left(u_{1}^{F}-u_{1}^{K}\right)
\end{aligned}
$$

Here, with reference to Figure 2.9, the superscripts indicate particular nodes.

It should be noted that Equation 2.31 relies on numerical data at twice the distance from the crack-front as Equation 2.30. It has been found in numerical experiments to be slightly less consistent with the results generated from the traction formula, Equation 2.29. This is to be expected; however, with good mesh design there is usually good agreement of the stress intensity factor results obtained using these formulae; they thus provide a good check on the results.

It should also be noted that the above formulas are based on the $1 / \sqrt{r}$ stress singularity which does not hold true when the crack-front intersects the free surface. 
Thus the stress intensity factor at that location should be used with some caution. The results from the displacement formula were presented throughout, unless stated otherwise.

\subsection{Numerical Examples - Fracture Problems}

To affirm the validity of the BEM for fracture mechanics problems, a number of test problems were analyzed. As a first example, a circular embedded crack in an infinite solid was modelled. Once this was validated, the problem of a thick-walled cylinder with a semi-elliptical crack as investigated by Tan \& Fenner (1979), as well as by Shim (1986), was analyzed and the results for the stress intensity factor are compared. Additionally, the same model was analyzed to obtain polynomial influence coefficients for the stress intensity factor and its application to an autofrettaged cylinder is demonstrated. Poisson's ratio was taken to be 0.3 throughout.

\subsubsection{Circular Embedded Crack in an Infinite Solid}

The well-known problem of a circular embedded crack in a theoretically infinite solid was analyzed as a preliminary test of the application of the BEM for crack problems. A one-eighth model was created due to the symmetry along the plane of the crack as well as the axisymmetry of the crack itself. Thus a quarter-circle crack was modelled on the corner of a cube face. To represent a theoretically infinite solid, the side of the cube was taken to be ten times the radius of the crack. The model was constrained from displacements normal to each of the three planes of symmetry. It was loaded with a tensile stress at the bottom surface in the direction normal to the crack face.

The mesh for the model has 229 nodes and 79 elements, as shown in Figure 2.10;

four elements across $90^{\circ}$ were placed along either side of the crack periphery. The exact analytical solution to the circular embedded crack problem is provided in Unger 
(1995)

$$
K_{I}=\frac{2}{\pi} \sigma \sqrt{\pi a}
$$

where $\sigma$ is the applied stress and $a$ is the radius of the penny-shaped crack. Table 2.2 shows the comparison of the results obtained using the traction and displacement formulas against the exact solution, Equation 2.32. It is evident that the traction formula is more accurate in this instance with a percent error below $1 \%$. The displacement formula results are between 2 and $5 \%$ lower than those from the traction formula and yield a percent error within 5.3\%. The nodes that produced a greater percent error with the displacement formula were located at midpoints and thus were twice the distance from the crack-front as the other nodes. The correlation between the traction and displacement formulas was sufficient to determine that there were no substantial errors in the data reduction and that the model was appropriately discretized, even if the mesh appears relatively coarse.

\subsubsection{Cylinder with Semi-Elliptical Crack}

It is apparent that if the results for an elliptical crack in a straight tubing could be reproduced to good accuracy, then the same modelling strategy would also be suitable for the analysis of curved tubing. Thus, a series of test models were analyzed to validate the method employed in solving for stress intensity factors using the BEM code employed, BIE3D5. Figure 2.11 shows the diametrical section of the physical problem. The radius ratio of the cylinder is denoted by $k=R_{2} / R_{1}$, and the semielliptical crack is defined by the elliptical aspect ratio, $b / a$, of its semi-major and semi-minor axes. The relative crack depth is defined by $b / W$, where $W=\left(R_{2}-R_{1}\right)$, and the position of points along the crack-front is defined by the angle $\phi$ as shown in the figure. 
Four test models were created and the results verified by comparison with previously published analyses by Shim (1986) who had also used a BEM code but had obtained the stress intensity factors by extrapolation techniques. The parameter combinations selected to test are: $k=2.5, b / W=0.2,0.4$ and $0.6 ; k=2, b / W=0.4 ;$ and all for which $b / a=0.8$.

Recognizing the two planes of symmetry, a quarter model was used to represent each case. One plane of symmetry is the diametrical plane containing the crack faces, and the other is the axial plane of the cylinder along the semi-minor axis of the semi-elliptical crack. In addition to the symmetrical constraints, the model was constrained using a plane strain end condition, as well as nodal constraints at each node $90^{\circ}$ to the axial plane of symmetry.

Figure 2.12 shows a typical mesh, in this instance for the crack depth of $b / W=0.4$, and $k=2.5$. All of the models were discretized with 8-node quadrilaterals and 6node triangles, ranging between 92 and 123 elements for each model. More elements were applied to the case with the smallest crack depth to ensure that there was an appropriate gradation in element size approaching the crack-front. It was determined that it was not necessary to apply more than one element across the wall thickness as the BEM is not particularly mesh-sensitive as long as a reasonable aspect ratio is maintained and neighbouring elements do not vary excessively in size. In all instances, $\nu=0.3$ and uniform hydrostatic pressure, $P$, was applied to all elements on the internal surface of the cylinder as well as the crack faces.

Due to the different mesh designs being used for this analysis from those previously employed by Shim (1986) and Tan \& Fenner (1980), the results are presented graphically. The stress intensity factors have been normalized as $K_{I} /(P \sqrt{\pi b})$. Figure 2.13 shows the results from the $k=2, b / W=0.4$ test case. It can be seen that there is good agreement between the test model results and the previously published results of Shim (1986), as well as Tan \& Fenner (1980). This good agreement is also seen for 
the $k=2.5, b / W=0.2,0.4$ and 0.6 cases, shown in Figures 2.14 to 2.16. The percent deviations remain below $3 \%$ in all instances, except for $k=2.5, b / W=0.4$ where they are larger but are still less than $5 \%$.

\subsection{Influence Function Method for Obtaining Stress Intensity Factors}

The influence function or influence coefficient method is an extension of the wellknown weight function method in fracture mechanics analysis; it allows stress intensity factors for a given cracked geometry to be obtained for various load cases without requiring a completely new analysis of the problem. The method is based on the principle of linear superposition. It allows a problem to be reduced to the summation of the stress analysis of the uncracked component, subjected to the same external loads, and the cracked component where the loading is only applied to the crack faces, as shown in Figure 2.17. Therefore,

$$
K_{I}=K_{I}^{\left({ }^{\prime}\right)}+K_{l}^{\left({ }^{\prime \prime}\right)}
$$

The loads acting on the crack faces are equal and opposite to the stresses that would exist on the crack face if the crack was not present. As $K_{I}^{\left({ }^{\prime}\right)}=0$, the solution for the stress intensity factor for the complete problem is reduced to that of the load applied directly to the crack faces in accordance with the uncracked stress distribution.

$$
K_{I}=K_{I}^{\left({ }^{\prime \prime}\right)}
$$

Assume the uncracked stress distribution due to external loads can be represented 
by a third degree polynomial as,

$$
\sigma_{r s}=A_{0}+A_{1} r_{s}+A_{2} r_{s}^{2}+A_{3} r_{s}^{3}
$$

where $r_{s}$ is a non-dimensionalized distance as shown in Figures 2.11, and $A_{i}$ represents the coefficients of the polynomial function with units of stress. It is possible to obtain stress intensity factors for the cracked problem in terms of polynomial influence coefficients, $K_{I}^{(i)}$. These influence coefficients are stress intensity factors corresponding to the problem with the crack faces subjected to the direct stress distribution as follows: uniform $\left(\sigma=-A_{0}\right)$; linear $\left(\sigma=-A_{1} r_{s}\right)$; quadratic $\left(\sigma=-A_{2} r_{s}^{2}\right)$; and cubic $\left(\sigma=-A_{3} r_{s}^{3}\right)$. The stress intensity factor for the problem subjected to the same arbitrary loading would be

$$
K_{I}=K_{I}^{(0)}+K_{I}^{(1)}+K_{I}^{(2)}+K_{I}^{(3)}
$$

\subsubsection{Numerical example 1}

The above case of $k=2, b / W=0.4$ was used with the same mesh to validate the application of polynomial influence coefficients to fracture mechanics problems. Uniform, linear, quadratic and cubic tractions are applied to the crack face, each normalized with $r_{s}$, as defined in Figure 2.11, set to unity at $r=b$ in each load case. The influence coefficients are determined from the computed traction and displacement data using Equations 2.29 and 2.30 and then normalizing by $A_{i}(b / W)^{i} \sqrt{\pi b}$; they are given in Table 2.3 .

After determining the influence coefficients, they were multiplied by the uncracked stress distribution for an internally pressurized cylinder to re-validate the stress intensity factors above. To this end, the well-known Lamé solution was approximated 
as a cubic polynomial as follows

$$
\sigma_{r s}=P\left(1.6735-2.29 r_{s}+2.0172 r_{s}^{2}-0.7312 r_{s}^{3}\right)
$$

The corresponding coefficients for the uncracked stress distribution in Equation 2.37 and the influence coefficients are multiplied together and summed to determine the stress intensity factors as follows for $\theta=90^{\circ}$,

$$
\begin{aligned}
& K_{I}^{(0)}=K_{I}^{(0) *} A_{0} P(b / W)^{0} \sqrt{\pi b}=0.658(1.6735 P) \sqrt{0.4 \pi}=1.234 P \\
& K_{I}^{(1)}=K_{I}^{(1) *} A_{1} P(b / W)^{1} \sqrt{\pi b}=0.119(-2.29 P)(0.4) \sqrt{0.4 \pi}=-0.128 P \\
& K_{I}^{(2)}=K_{I}^{(2) *} \Lambda_{2} P(b / W)^{2} \sqrt{\pi b}=0.046(2.0172 P)(0.4)^{2} \sqrt{0.4 \pi}=0.017 P \\
& K_{I}^{(3)}=K_{I}^{(3) *} A_{3} P(b / W)^{3} \sqrt{\pi b}=0.024(-0.7312 P)(0.4)^{3} \sqrt{0.4 \pi}=-.001 P
\end{aligned}
$$

For an internally pressurized cylinder, with pressure acting on the crack faces, an additional uniform stress of $\mathrm{P}$ is applied,

$$
\begin{aligned}
K_{I} & =K_{I}^{(0) *} P \sqrt{\pi b}+\sum_{i=0}^{3} K_{I}^{(i)} \\
& =0.658 P \sqrt{0.4 \pi}+(1.234-0.128+0.017-0.001) P \\
& =1.867 P \\
K_{I} /(P \sqrt{\pi b}) & =1.665
\end{aligned}
$$

The stress intensity factors around the crack periphery produced by this method are compared to the results obtained by Tan \& Shim (1986) in Figure 2.18. The deviation between the two sets of results shown are less than $4 \%$, and is $1.7 \%$ at the free surface. In addition, the stress intensity factors calculated here using the influence coefficient method deviate less than $2.5 \%$ from the results that were calculated by the direct method presented above, as shown in Table 2.4. The agreement shown between 
the influence coefficient method and the direct BEM, as well as the stress intensity factors and influence coefficients presented here and those previously published is sufficient to validate the use of influence coefficients for fracture mechanics problems, particularly that of internally pressurized tubing.

\subsubsection{Numerical example 2}

As another example of the versatility of the influence function method, the influence coefficients obtained from the BEM analyses are applied to the problem of an autofrettaged cylinder with the same semi-elliptical crack. The cases of the cracked cylinder with $k=2, b / W=0.4$ and which has been autofrettaged to $20 \%$ and $50 \%$ overstrain were considered.

Autofrettage is a process whereby favourable residual stresses are generated in a component by loading it into the plastic range. The depth of the plastic zone, terminating at the elastic-plastic interface, is shown as $n R_{1}$ in Figure 2.19. For a cylinder, the internal pressure to be applied to result in a plastic zone with a given radial depth, $n R_{1}$, can be determined from

$$
P=\sigma_{y}\left[\log _{e} n+\frac{k^{2}-n^{2}}{2 k^{2}}\right]
$$

where $\sigma_{y}$ is the yield stress. This depth is often quantified as the percent overstrain, which is defined as the percentage of the wall thickness which has plastically deformed,

$$
\% \text { Overstrain }=\frac{n-1}{k-1} \times 100 \%
$$

The residual hoop stress distribution in a cylinder due to partial autofrettage, assuming plane strain end conditions, the von Mises yield criterion and that the plastic 
deformation is strain-history independent, can be expressed as follows, Hill (1950)

$$
\begin{gathered}
\sigma_{\theta}=\frac{2}{\sqrt{3}} \sigma_{y}\left[\frac{1}{k^{2}-1}\left(1+\frac{R_{2}^{2}}{r^{2}}\right)\left(\frac{n^{2}-k^{2}}{2 k^{2}}-\log _{e} n\right)+\frac{n^{2}+k^{2}}{2 k^{2}}-\log _{e} \frac{n R_{1}}{r}\right] \\
R_{1} \leq r \leq n R_{1} \\
\sigma_{\theta}=\frac{2}{\sqrt{3}} \sigma_{y}\left(1+\frac{R_{2}^{2}}{r^{2}}\right)\left[\frac{n^{2}}{2 k^{2}}+\frac{1}{k^{2}-1}\left(\frac{n^{2}-k^{2}}{2 k^{2}}-\log _{e} n\right)\right], \\
n R_{1} \leq r \leq R_{2}
\end{gathered}
$$

A cubic polynomial can sufficiently describe the residual stress distribution in each of the elastic and plastic regions. When $n R_{1}$ is greater than the crack depth, $b$, the crack faces are fully plastic and the stress distribution across this region can be expressed as a single cubic polynomial as in Equation 2.35. Thus, influence coefficients may be applied to compute the stress intensity factors. In the circumstance when $n R_{1}$ is less than $b$ however, a cubic polynomial is not sufficient to describe the stress distribution across the crack faces. Thus, when the elastic-plastic interface occurred within the crack depth, the solution of the stress intensity factors was computed directly from the BEM. The same BEM mesh was used, with 95 quadratic isoparametric elements and 273 nodes.

The stress distribution to fit the polynomial Equation 2.35 was obtained using a commercial finite element analysis software, ABAQUS. This was not really necessary for this particular test model due to the exact solution available as given by Equations 2.40 and 2.41. However, there is no available theoretical solution for the curved tubing analyses to follow and thus this opportunity was availed upon to check the validity of the output of the FEM code.

A model of 20 -node bricks was created and loaded until $20 \%$ of the wall thickness had yielded. The stresses recorded at this pressure were summed with the negative of the stresses obtained from subjecting the linear elastic cylinder to a uniform internal 
pressure equivalent to that which was applied to cause yielding to simulate linear unloading. The result of this summation was the residual stress in the cylinder. This residual stress was then fit with cubic polynomials to define the elastic and plastic regions. This process was repeated for a $50 \%$ overstrain.

Figure 2.20 shows the curves for the normalized residual hoop stress, $\sigma_{\theta} / \sigma_{y}$, across the cylinder wall obtained from ABAQUS and the exact solution at $50 \%$ overstrain. It can be seen that there is excellent agreement for results in the plastic region and a slight difference in the curves for the stresses across the wall section which remained elastic.

The stress intensity factors for the $20 \%$ overstrain case, where $n R_{1}$ is less than $b$, were computed directly. The polynomials determined from ABAQUS to describe both regions were used to apply tractions across the crack face. The output of BIE3D5 was then post-processed and the stress intensity factors determined directly. The stress intensity factors were normalized by $K_{I} /\left[\sigma_{y} A_{i}(b / W)^{i} \sqrt{\pi b}\right]$ to preserve significant decimal places and compared to the published results by Shim (1986). They are shown plotted in Figure 2.21.

The solution of the stress intensity factors at $50 \%$ overstrain was computed using the influence coefficients provided in Table 2.3, similar to the approach used for the pressurized cylinder. These results were then normalized and compared to those by Shim (1986), as shown in Figure 2.22.

From Figures 2.21 and 2.22 the solutions obtained have good agreement with those previously published. This is especially evident in Figure 2.22 which shows $50 \%$ overstrain and thus was calculated using the influence coefficients in Table 2.3 . The use of percent error here would be misleading due to the fact the data vary over three orders of magnitude. Figure 2.21 also shows reasonable agreement with the available solution given by Shim (1986). The slight increase in discrepancy seen in this plot can be attributed to the stress distribution determined from ABAQUS, as 
FEM was found to be very mesh sensitive as well as from fitting the curves into cubic polynomials.

\subsection{Conclusions}

The boundary element method can be used efficiently for linear elastic fracture mechanics problems. It has been validated for determining stress intensity factors both directly and with the use of polynomial influence coefficients for an internally pressurized thick-walled cylinder. These influence coefficients may be applied to any load case for the same crack geometry and this was verified for the cylinder under internal pressure and for a partially autofrettaged cylinder with a semi-elliptical crack. As the cylinder test models have all been validated, a more complicated geometry, namely a curved tubing, will be analyzed next. 
Table 2.1: Variation of stress concentration factor at side of circular cylindrical hole in infinite plate of finite thickness with position through the plate thickness

\begin{tabular}{|c|l|l|c|c|}
\hline \multirow{2}{*}{$z / c$} & \multicolumn{4}{|c|}{$\sigma_{y} / \sigma$} \\
\cline { 2 - 5 } & $\begin{array}{l}\text { Sternberg \& } \\
\text { Sadowsky (1949) }\end{array}$ & $\begin{array}{l}\text { Tan \& Fenner } \\
(1978)\end{array}$ & BEM & FEM \\
\hline 0 & 3.11 & 3.00 & 3.07 & 3.10 \\
1 & 2.76 & 2.60 & 2.66 & 2.70 \\
\hline
\end{tabular}

Table 2.2: Comparison of normalized stress intensity factor, $K_{I}^{*}=K_{I} /\left(\frac{2}{\pi} \sigma \sqrt{\pi a}\right)$, for the penny-shaped crack problem: $\left(K_{I}^{*}\right)_{T}$ - Traction formula result; $\left(K_{I}^{*}\right)_{D}$ - Displacement formula result; Exact solution $K_{I}^{*}=1.000$

\begin{tabular}{|c|c|c|c|c|}
\hline \hline$\theta$ & $\left(K_{J}^{*}\right)_{T}$ & $\%$ Error & $\left(K_{J}^{*}\right)_{D}$ & \% Error \\
\hline \hline $0^{\circ}$ & 1.0081 & 0.812 & 1.0274 & 2.741 \\
$11.25^{\circ}$ & 0.9997 & -0.031 & 1.0513 & 5.132 \\
$22.5^{\circ}$ & 1.0060 & 0.601 & 1.0270 & 2.700 \\
$33.75^{\circ}$ & 1.0046 & 0.460 & 1.0524 & 5.244 \\
$45^{\circ}$ & 1.0060 & 0.601 & 1.0275 & 2.751 \\
$56.25^{\circ}$ & 1.0046 & 0.460 & 1.0523 & 5.233 \\
$67.5^{\circ}$ & 1.0060 & 0.601 & 1.0268 & 2.685 \\
$78.75^{\circ}$ & 0.9997 & -0.031 & 1.0510 & 5.102 \\
$90^{\circ}$ & 1.0074 & 0.741 & 1.0283 & 2.832 \\
\hline
\end{tabular}


Table 2.3: Normalized influence coefficients, $K_{I}^{(i) *}=K_{I}^{(i)} /\left[A_{i}(b / W)^{i} \sqrt{\pi b}\right]$, for $k=2$, $b / W=0.4$

\begin{tabular}{|l|c|c|c|c|c|c|c|c|c|}
\hline \multirow{2}{*}{$K_{I}^{(i) *}$} & \multicolumn{7}{|c|}{$\phi$} \\
\cline { 2 - 9 } & $0^{\circ}$ & $11.25^{\circ}$ & $22.5^{\circ}$ & $33.75^{\circ}$ & $45^{\circ}$ & $56.25^{\circ}$ & $67.5^{\circ}$ & $78.75^{\circ}$ & $90^{\circ}$ \\
\hline \hline$K_{I}^{(0) *}$ & 0.693 & 0.679 & 0.691 & 0.669 & 0.672 & 0.645 & 0.649 & 0.647 & 0.658 \\
$K_{I}^{(1) *}$ & 0.449 & 0.405 & 0.429 & 0.364 & 0.356 & 0.277 & 0.235 & 0.163 & 0.119 \\
$K_{I}^{(2) *}$ & 0.345 & 0.288 & 0.315 & 0.237 & 0.223 & 0.145 & 0.107 & 0.064 & 0.046 \\
$K_{I}^{(3) *}$ & 0.283 & 0.219 & 0.247 & 0.165 & 0.150 & 0.084 & 0.055 & 0.033 & 0.024 \\
\hline \hline
\end{tabular}

Table 2.4: Comparison of normalized stress intensity factors, $K_{I} /(P \sqrt{\pi b})$, for $k=2$, $b / W=0.4:$ IC - Influence coefficient method; $\triangle \%$ - \% difference

\begin{tabular}{|c|c|c|c|c|c|c|c|c|c|}
\hline \multirow{2}{*}{ Method } & \multicolumn{10}{|c|}{$\phi$} \\
\cline { 2 - 9 } & $0^{\circ}$ & $11.25^{\circ}$ & $22.5^{\circ}$ & $33.75^{\circ}$ & $45^{\circ}$ & $56.25^{\circ}$ & $67.5^{\circ}$ & $78.75^{\circ}$ & $90^{\circ}$ \\
\hline \hline Direct & 1.523 & 1.509 & 1.516 & 1.506 & 1.518 & 1.499 & 1.536 & 1.605 & 1.707 \\
IC & 1.540 & 1.527 & 1.546 & 1.523 & 1.536 & 1.513 & 1.550 & 1.600 & 1.665 \\
$\Delta \%$ & 1.130 & 1.178 & 1.997 & 1.143 & 1.184 & 0.952 & 0.954 & 0.303 & 2.440 \\
\hline \hline
\end{tabular}




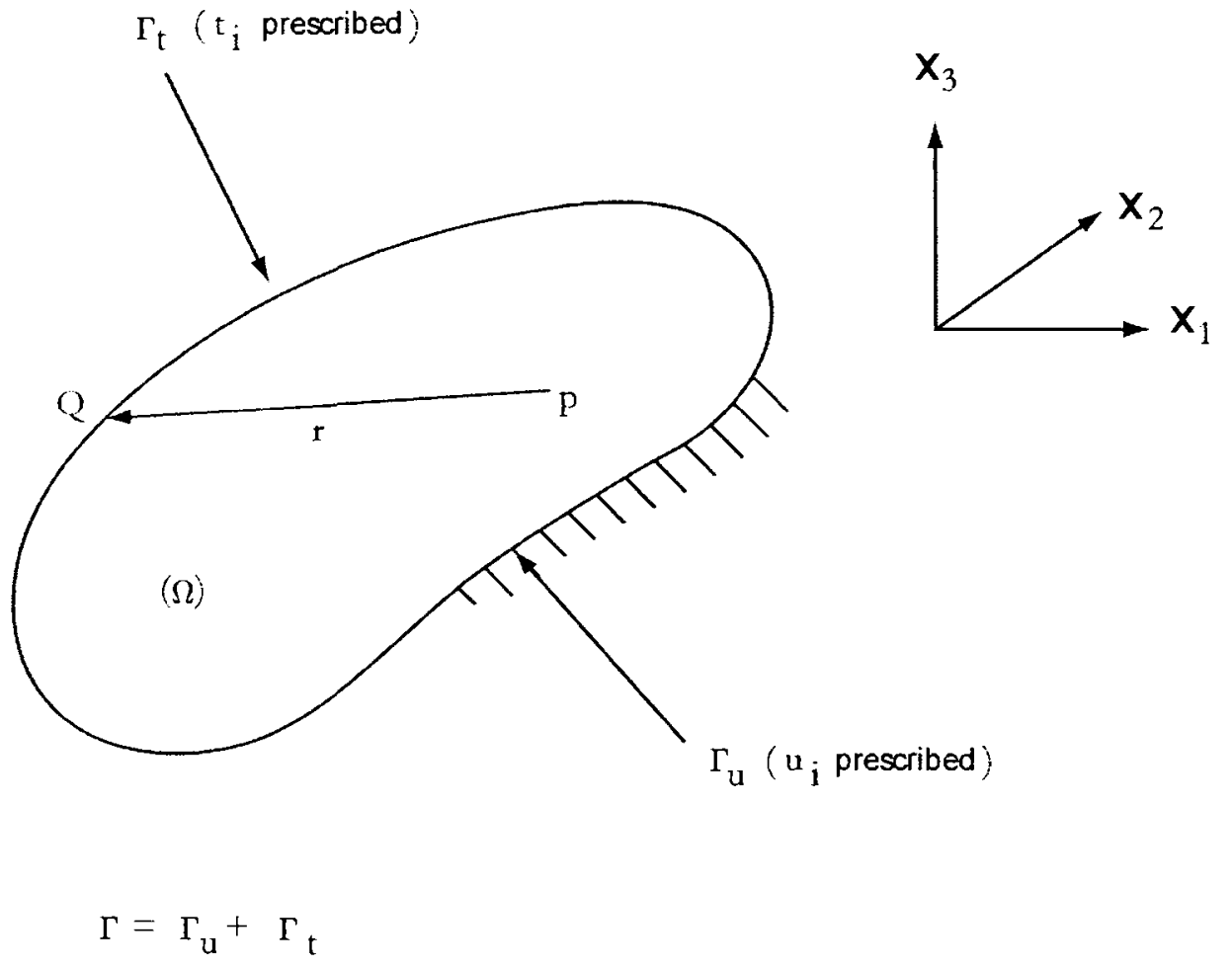

Figure 2.1: Boundary value problem in elastostatics 


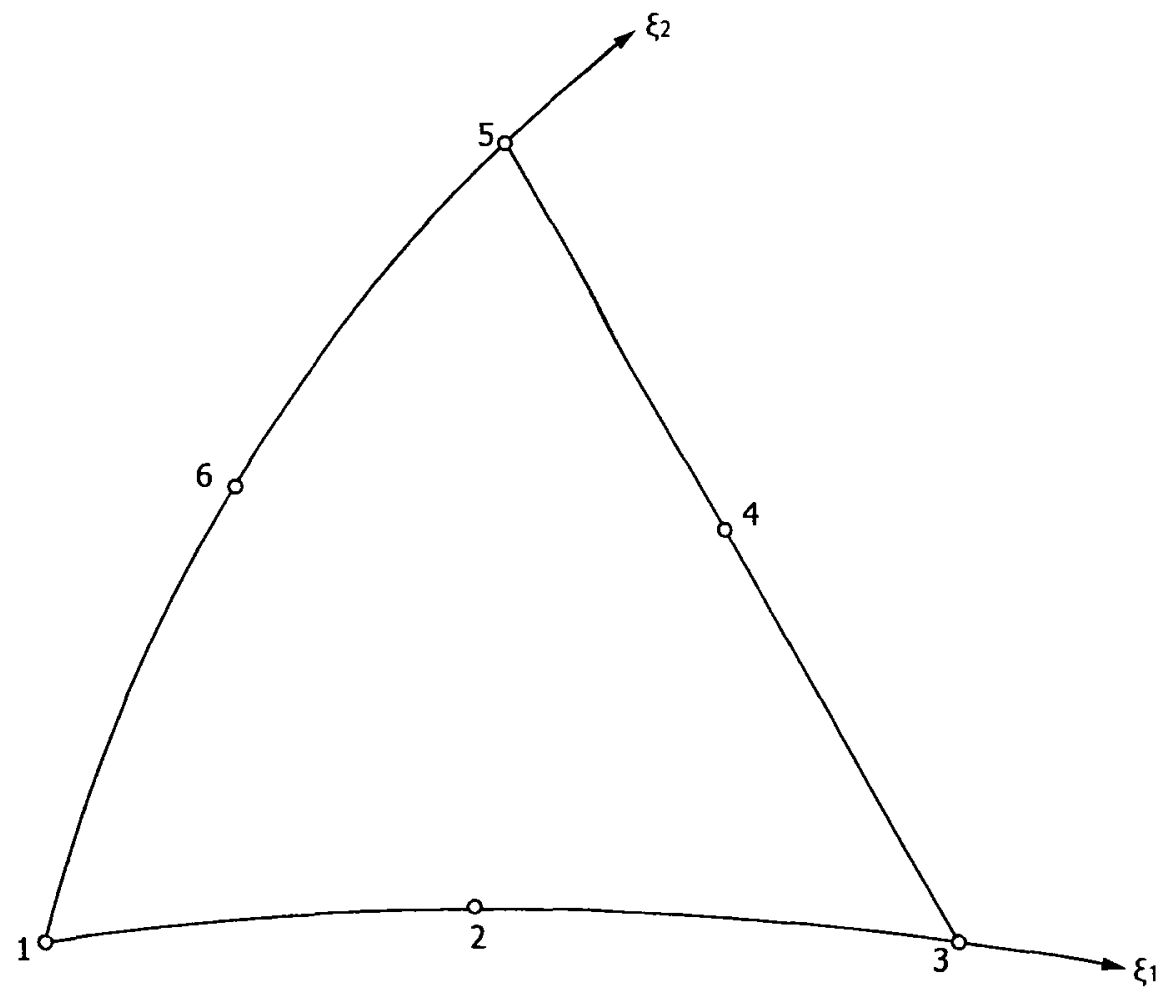

Figure 2.2: Six node triangular element 


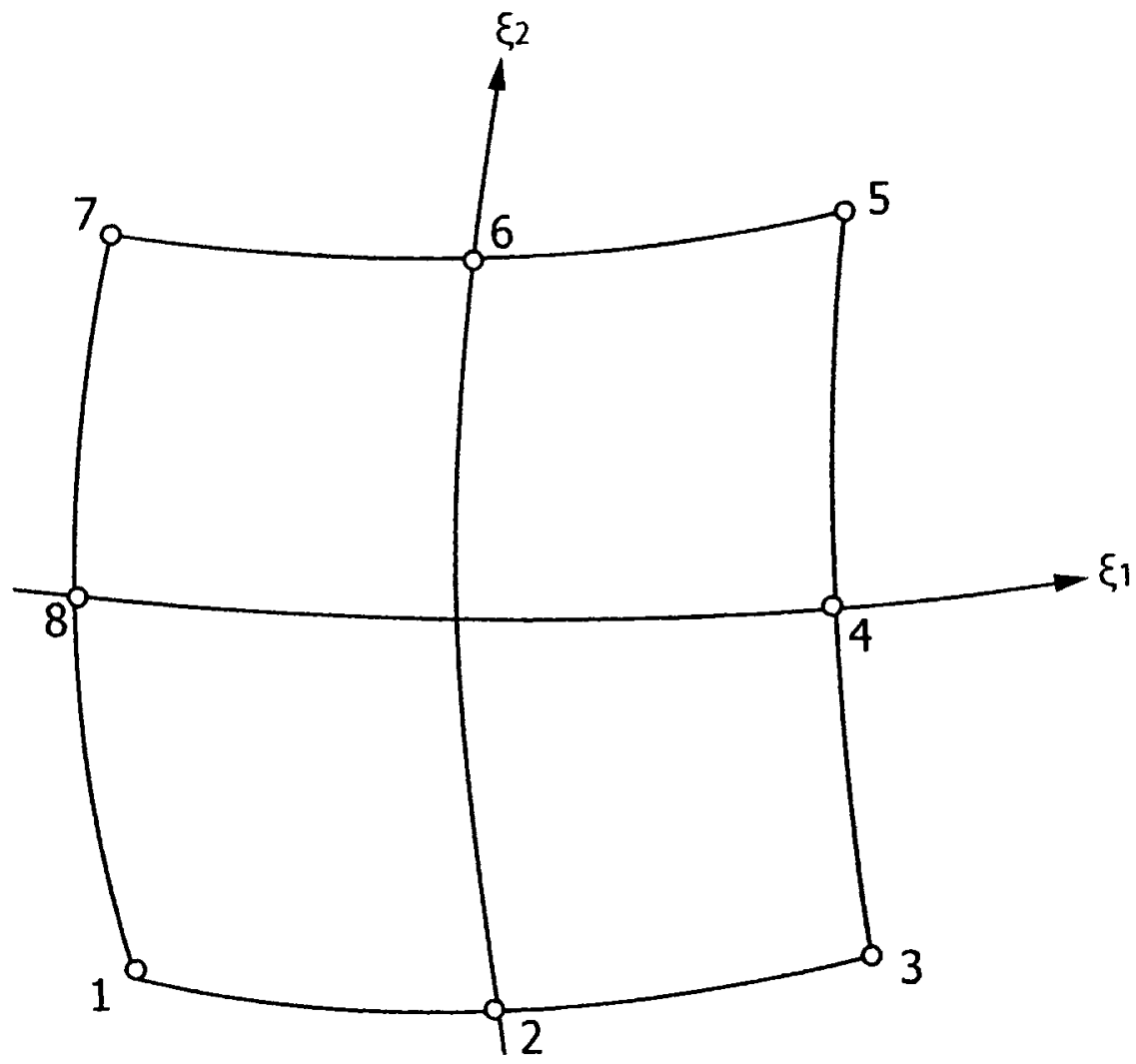

Figure 2.3: Eight node quadrilateral element 


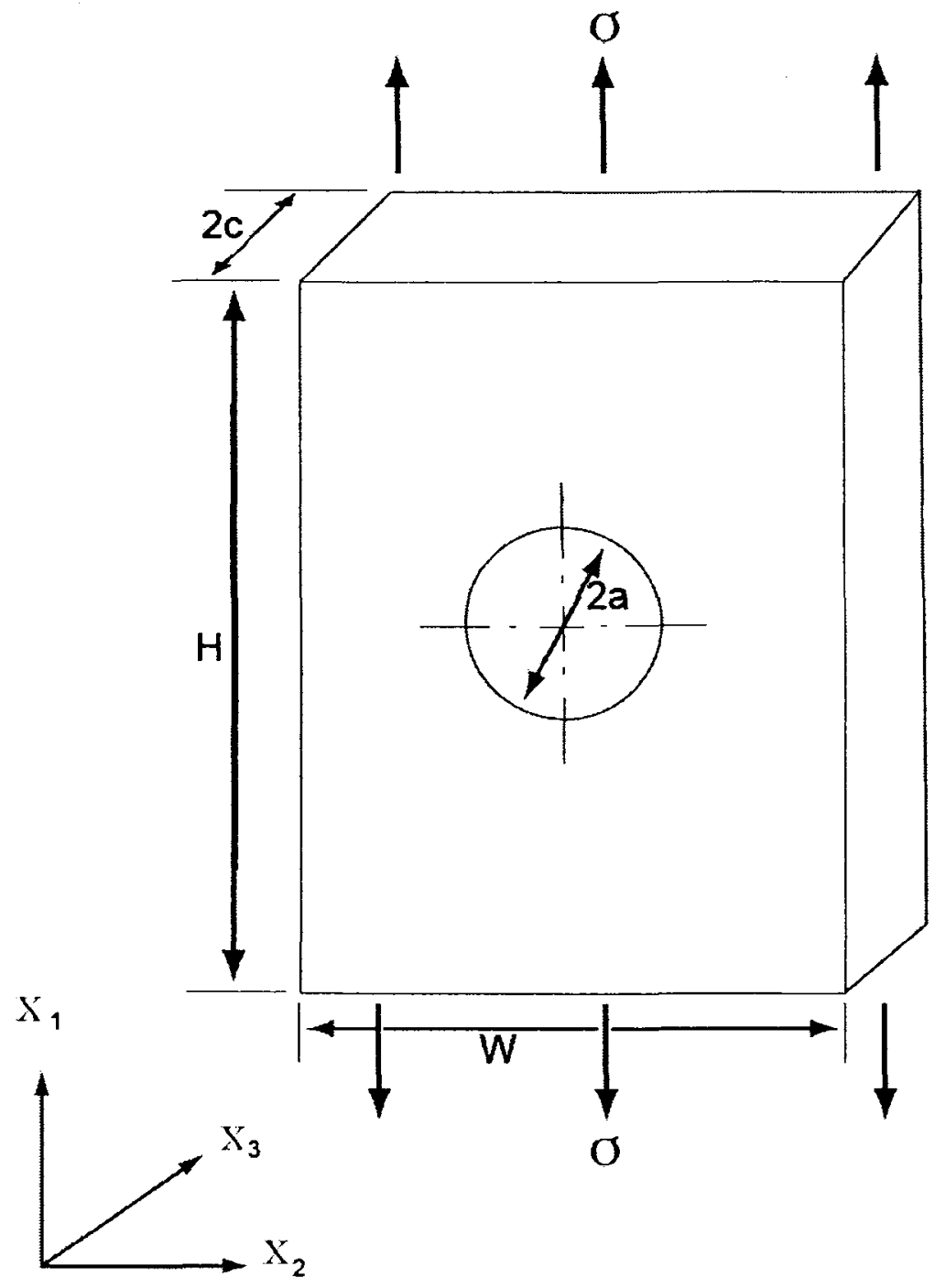

Figure 2.4: Circular hole in an "infinite" plate of finite thickness 


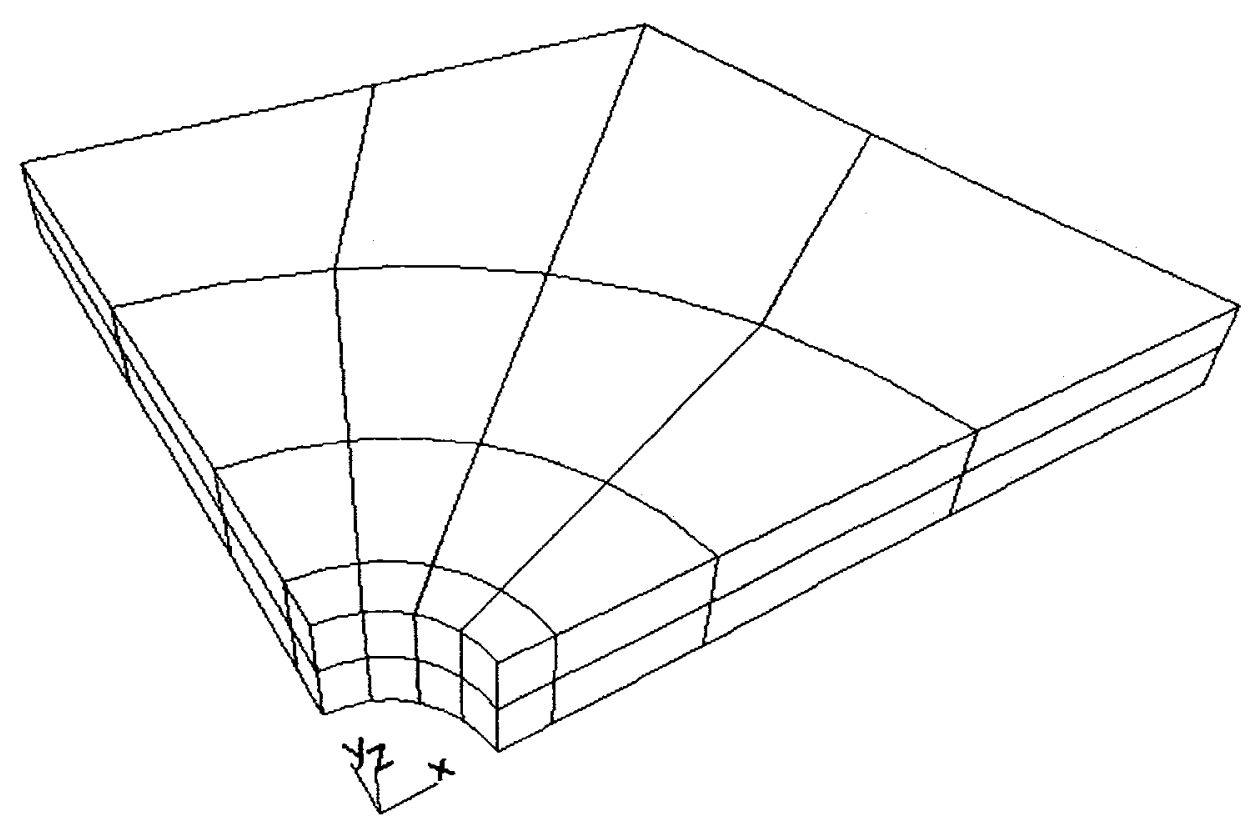

(a) BEM mesh

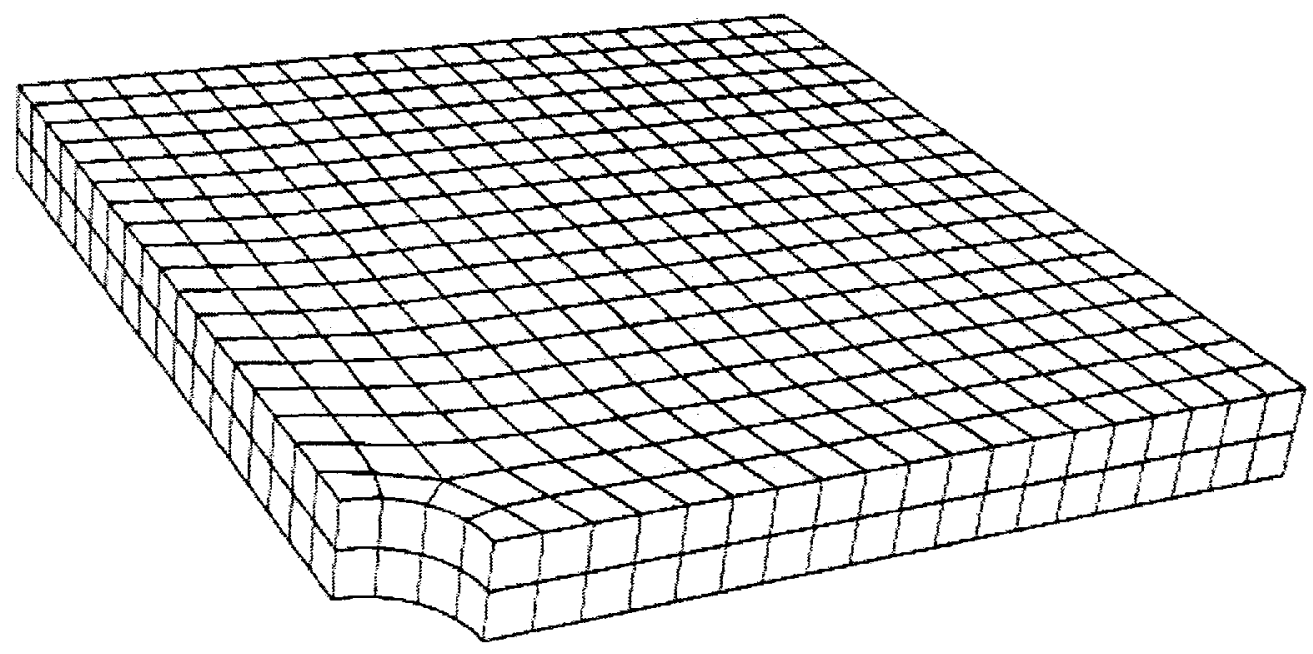

$V^{2}$

(b) FEM mesh

Figure 2.5: Discretization of the problem of a circular hole in an "infinite" plate of finite thickness 


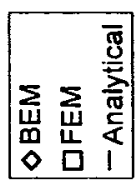

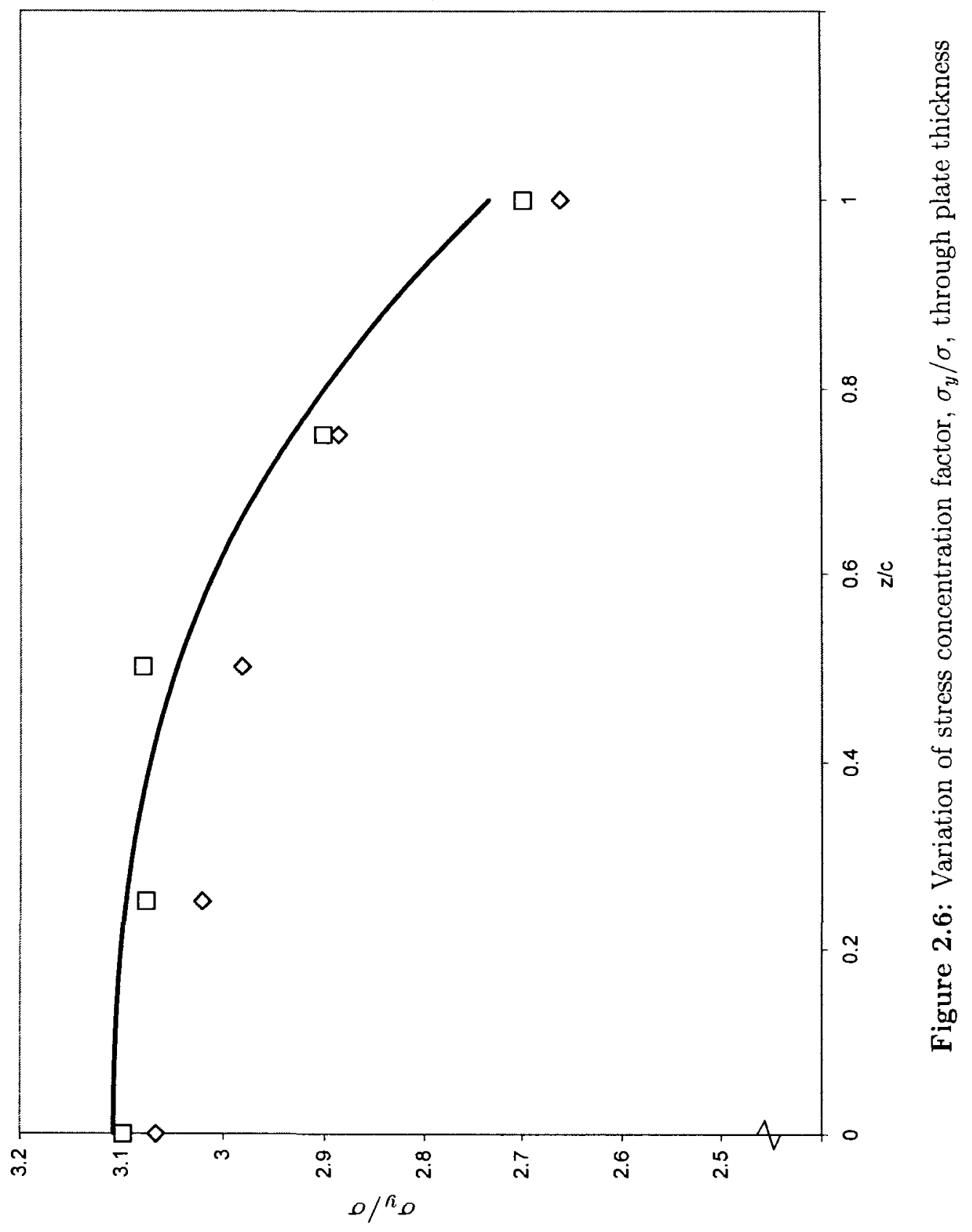




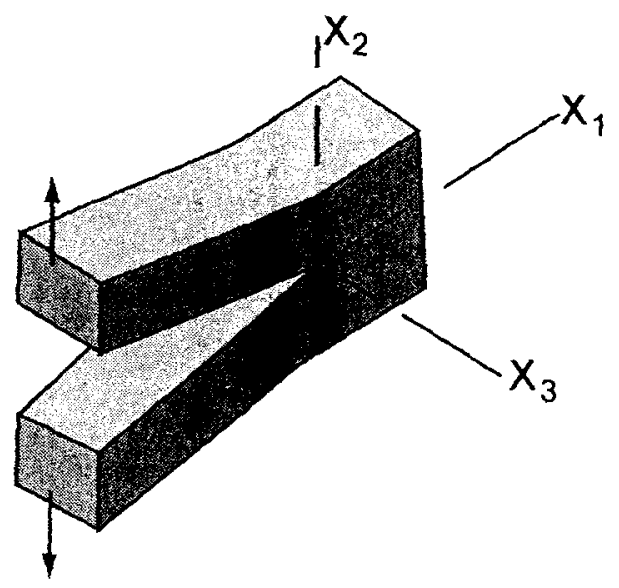

(a) Mode I

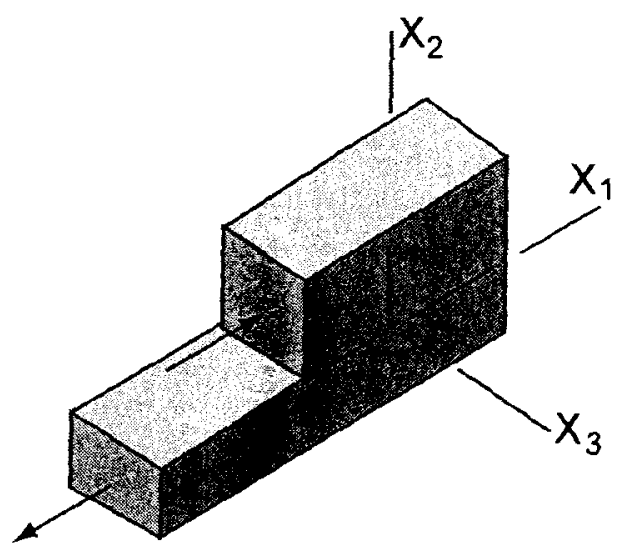

(b) Mode II

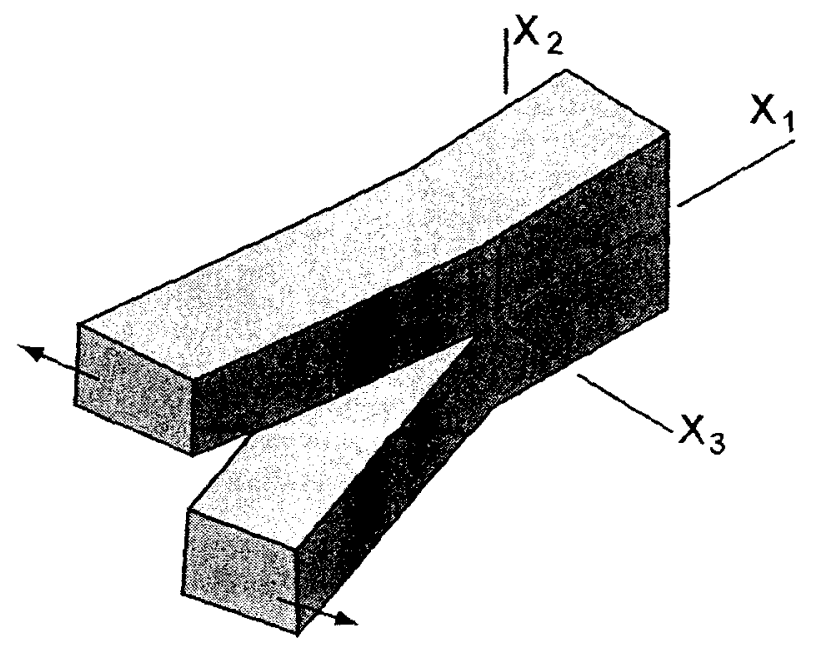

(c) Mode III

Figure 2.7: Three displacement modes for crack surfaces 


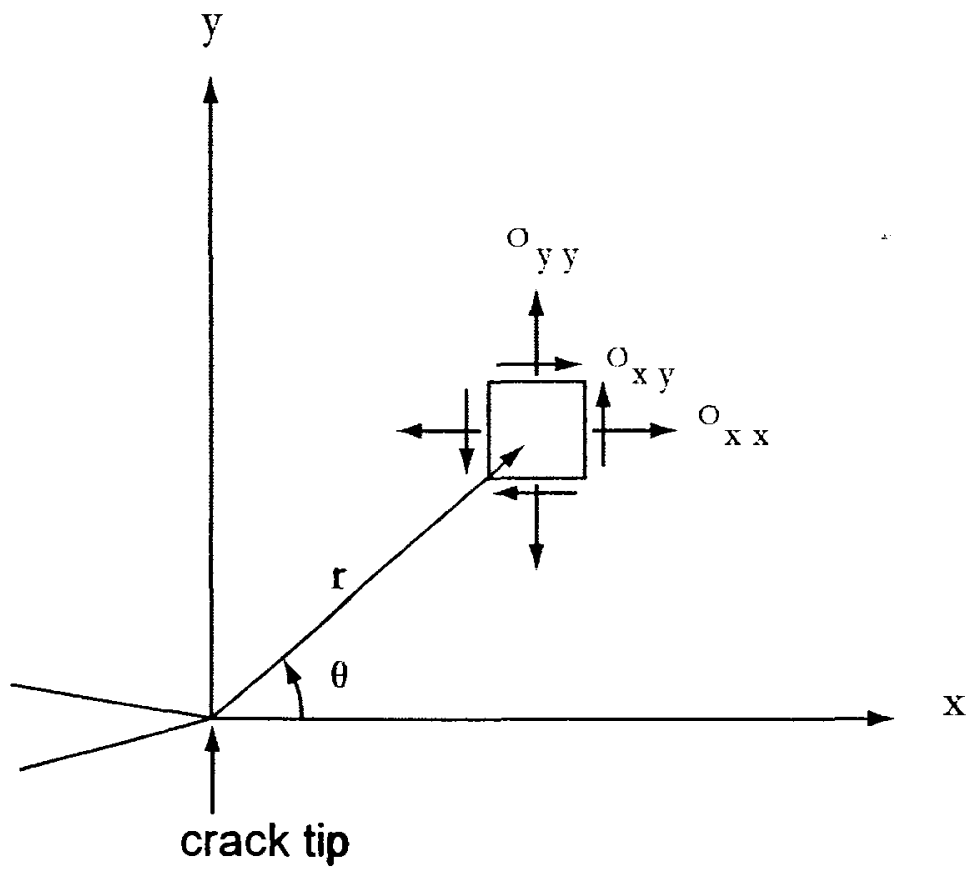

Figure 2.8: Crack-tip stresses shown with the Cartesian coordinate system 


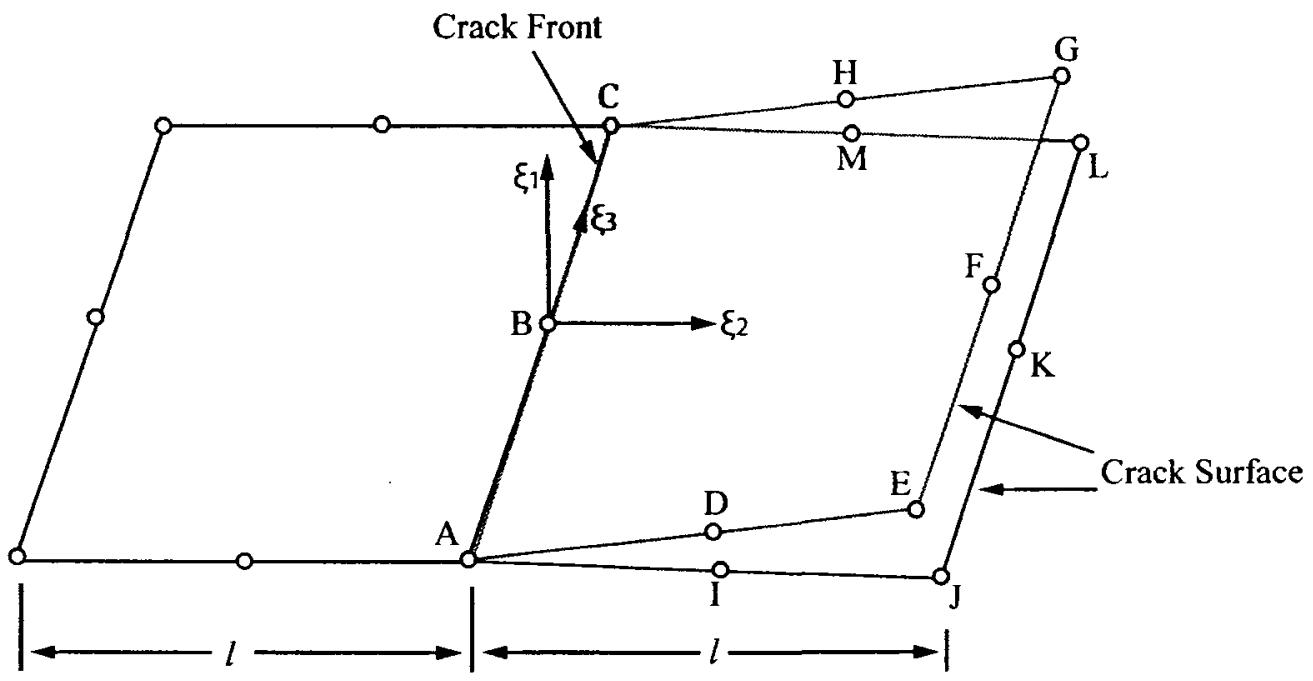

Figure 2.9: Crack-front element 


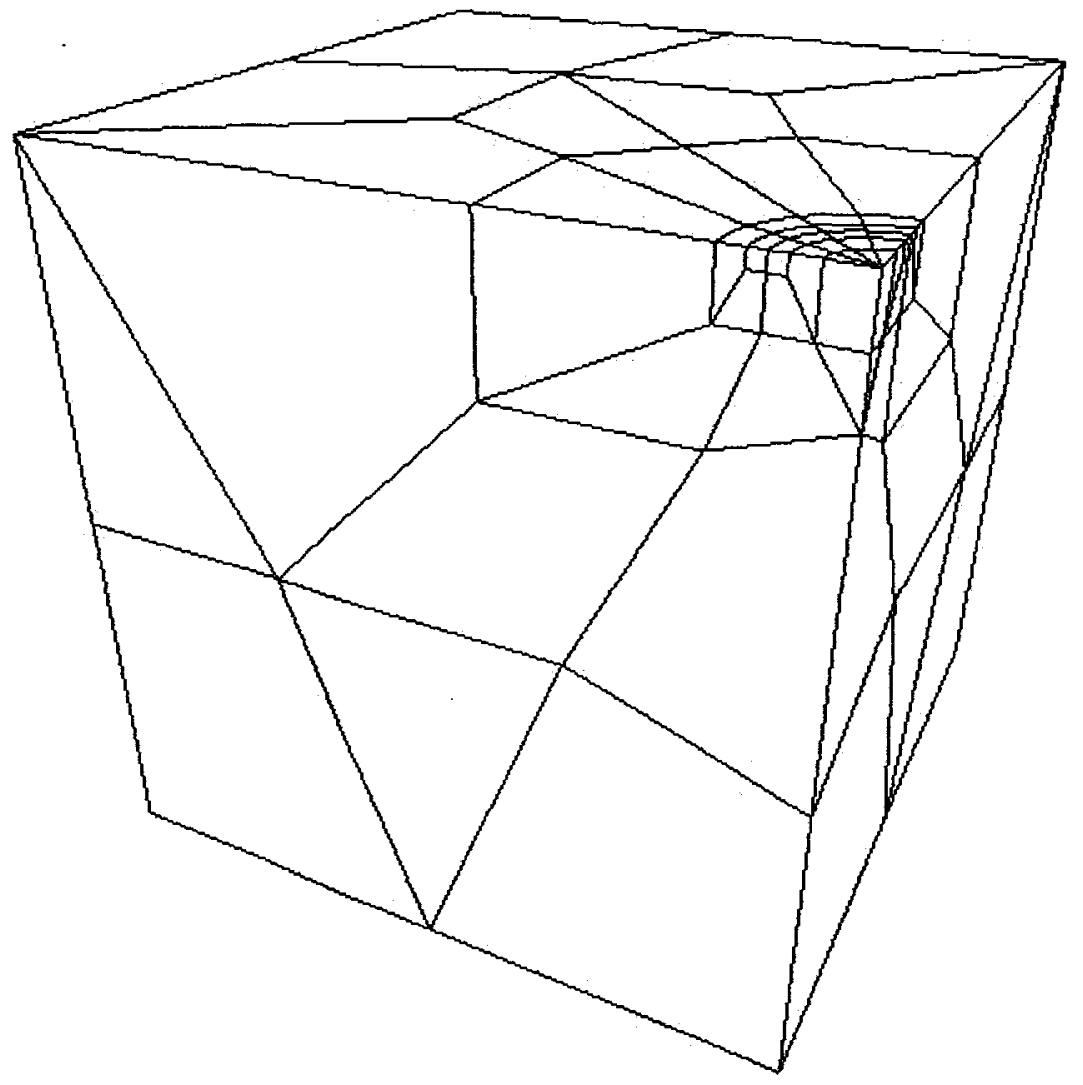

Figure 2.10: BEM mesh for problem of a circular embedded crack in an "infinite" solid 


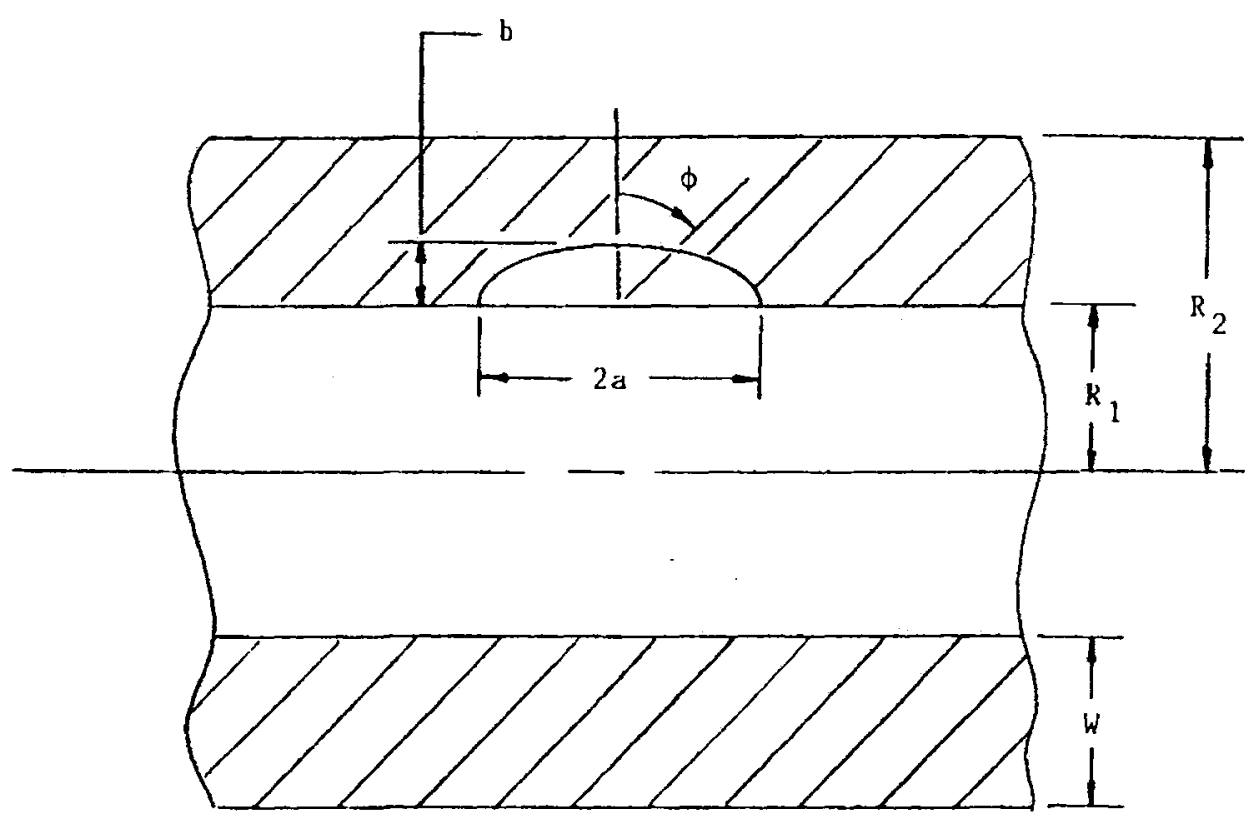

Figure 2.11: Semi-elliptical surface crack in a thick-walled cylinder 


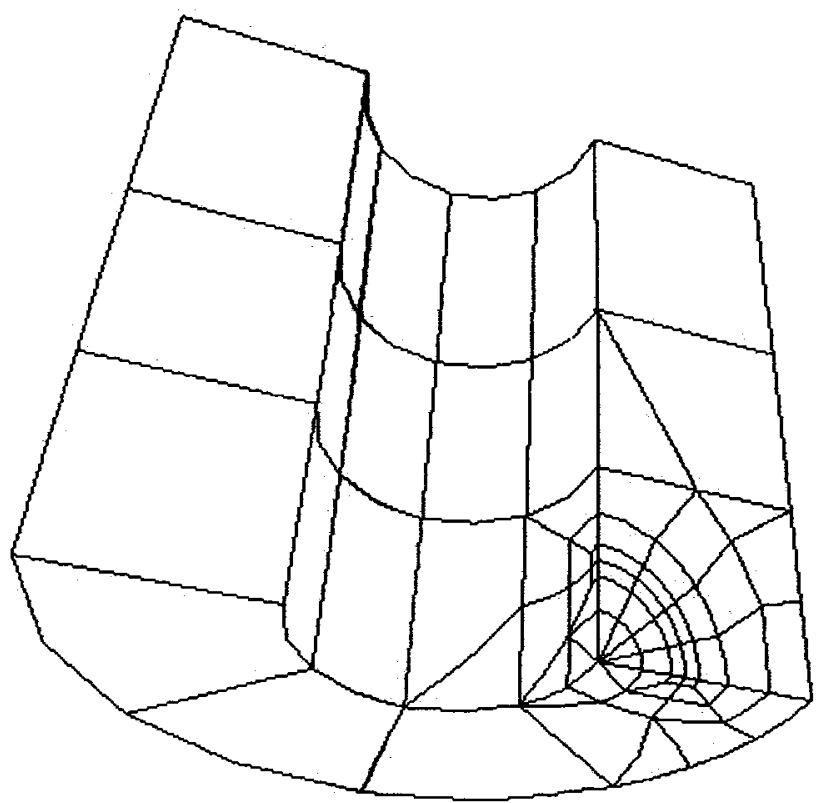

Figure 2.12: BEM mesh for cylinder with an elliptical crack, $k=2.5, b / W=0.4$ 


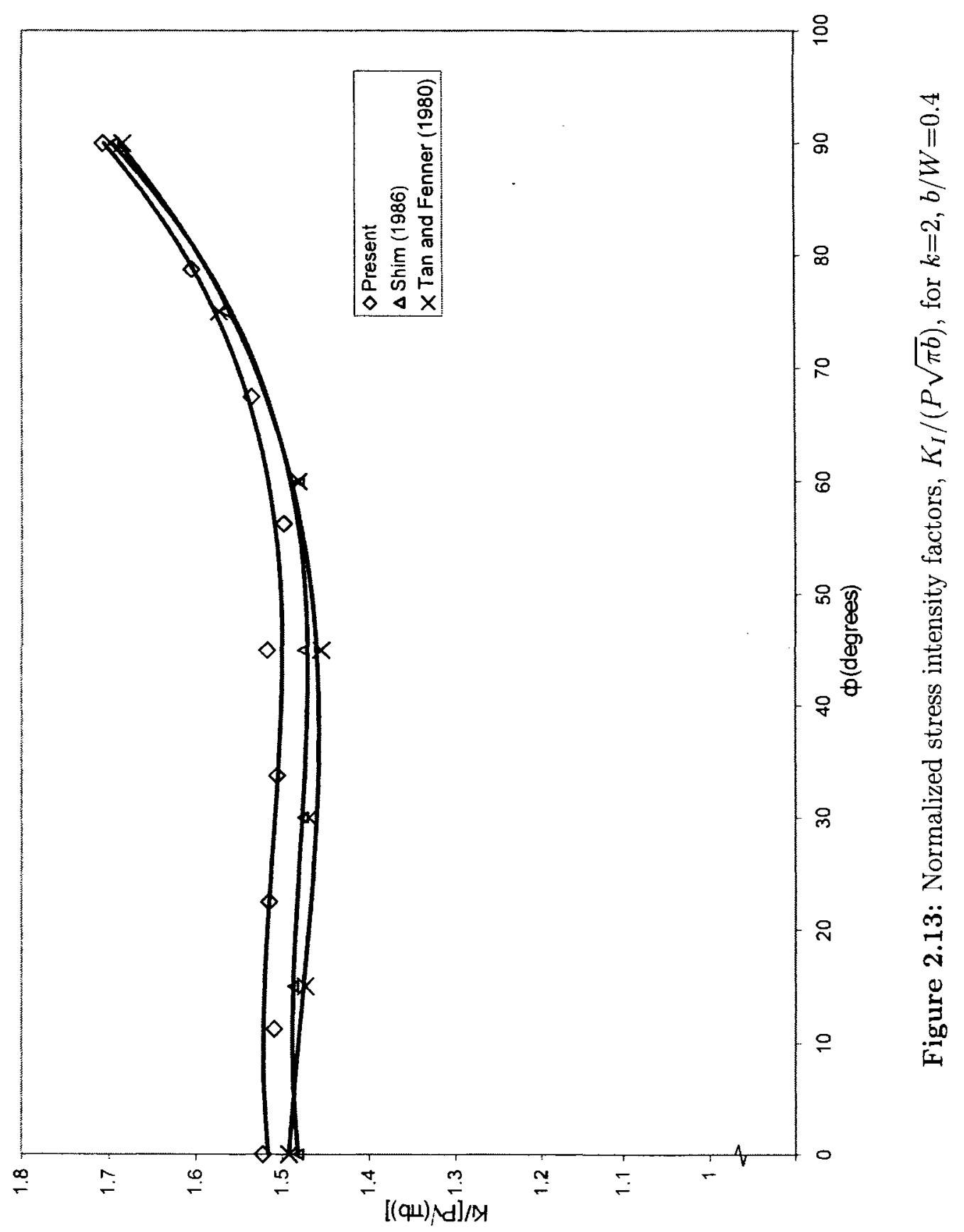




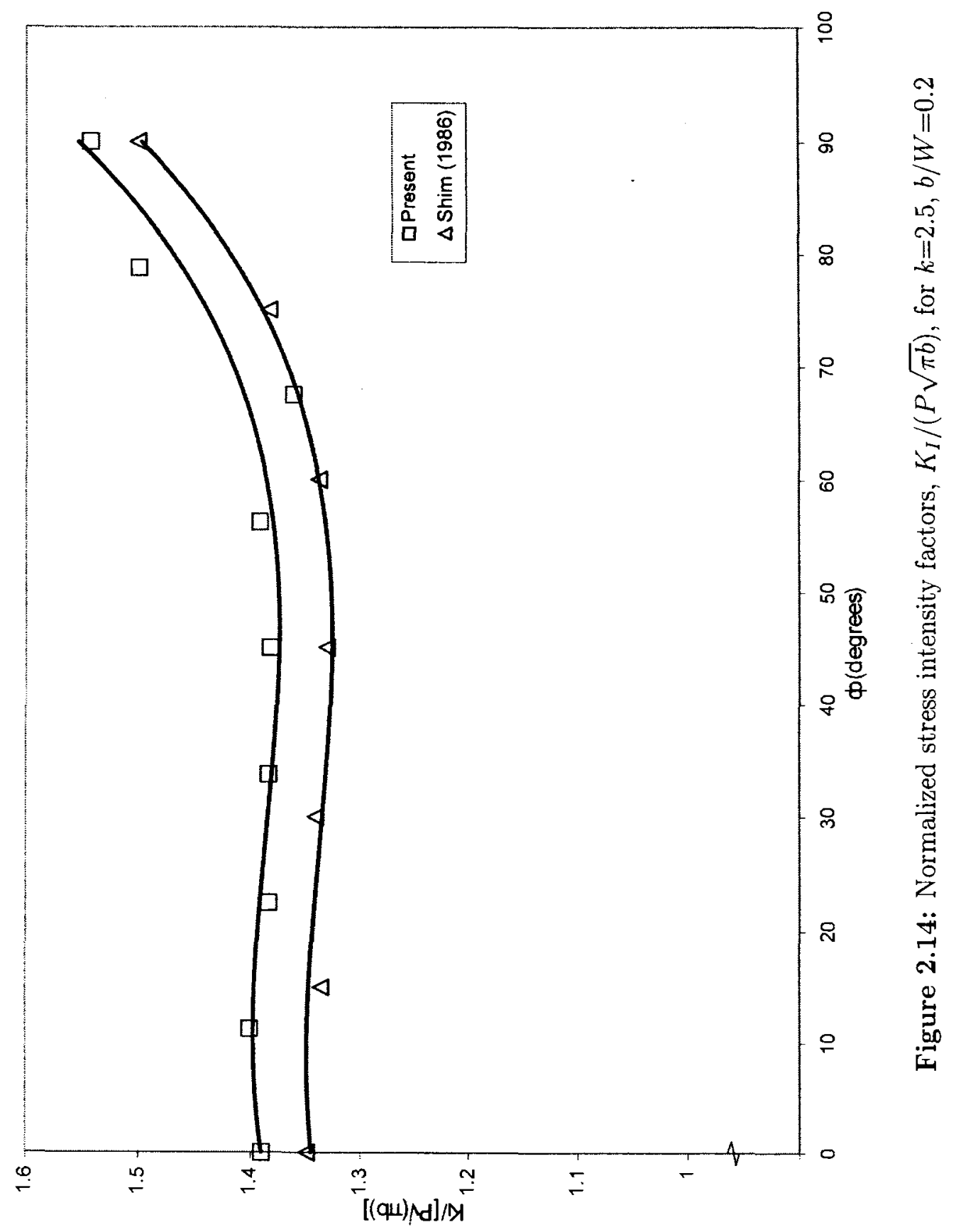




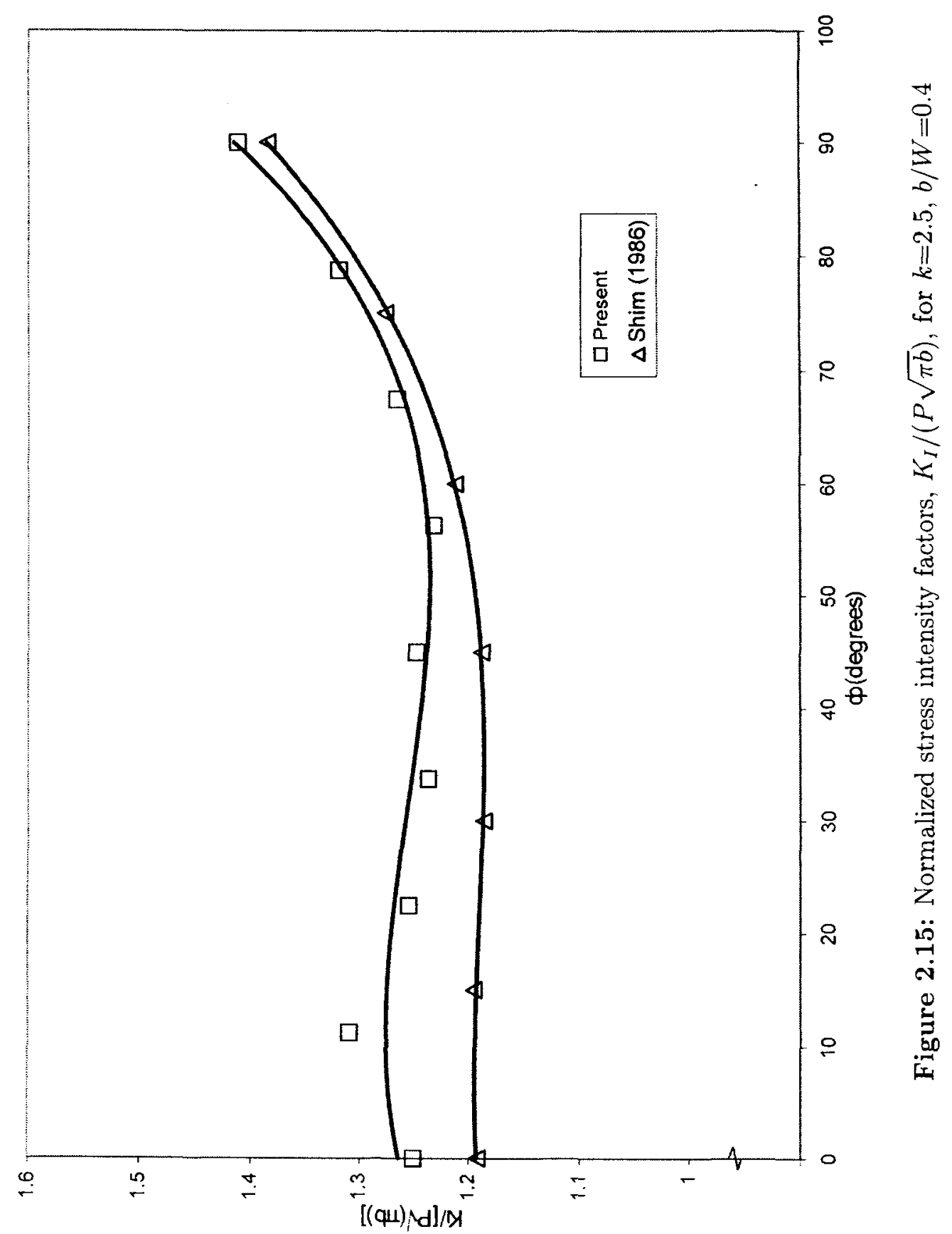




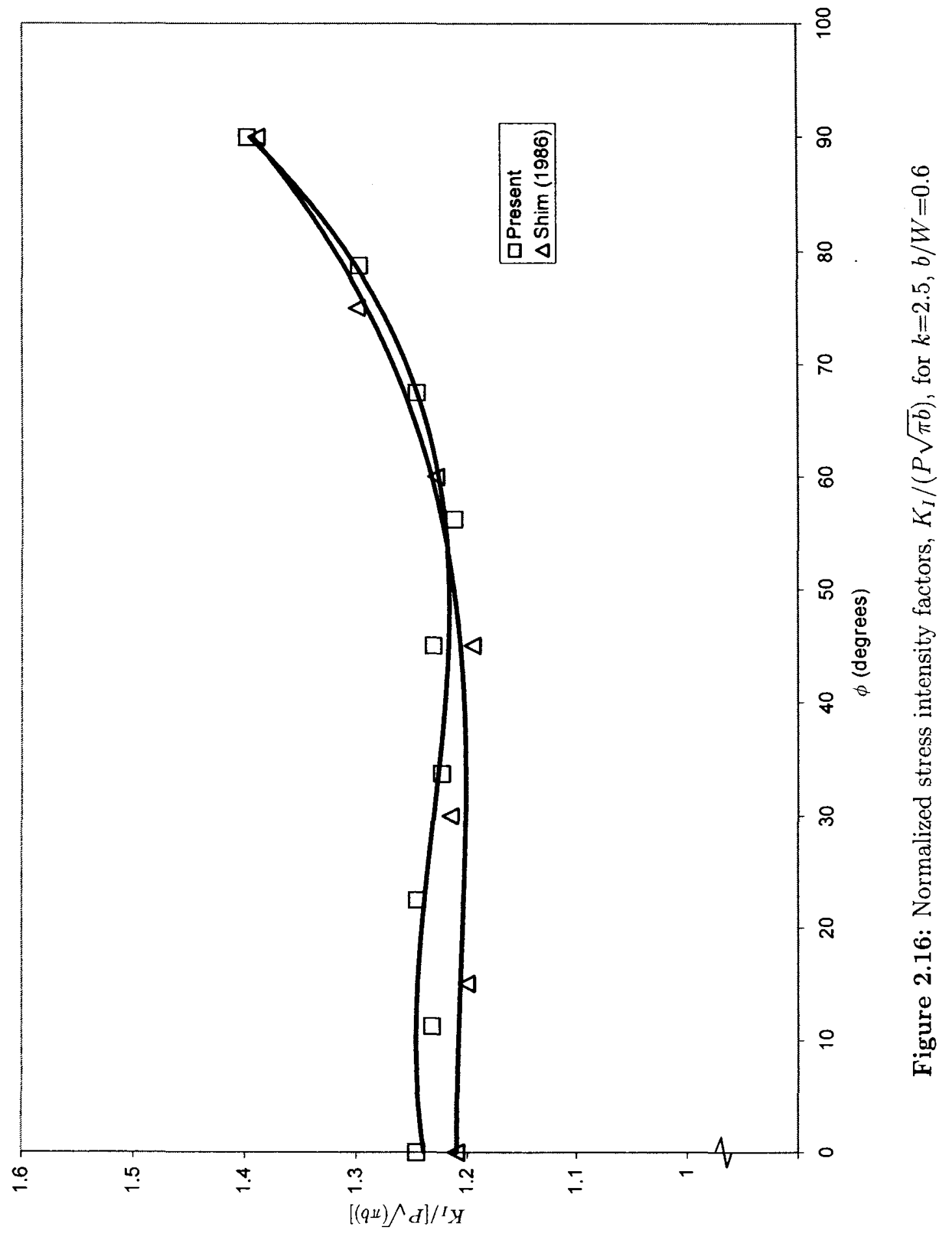




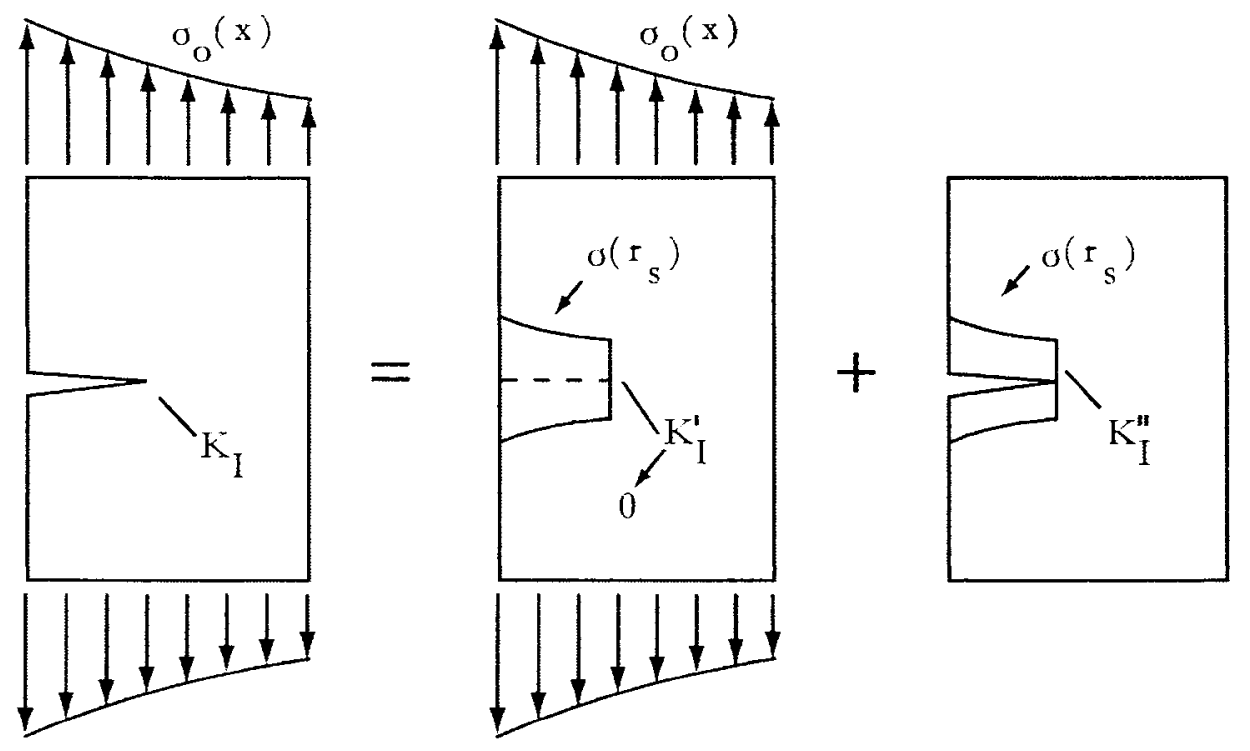

Figure 2.17: Principle of the weight function method 


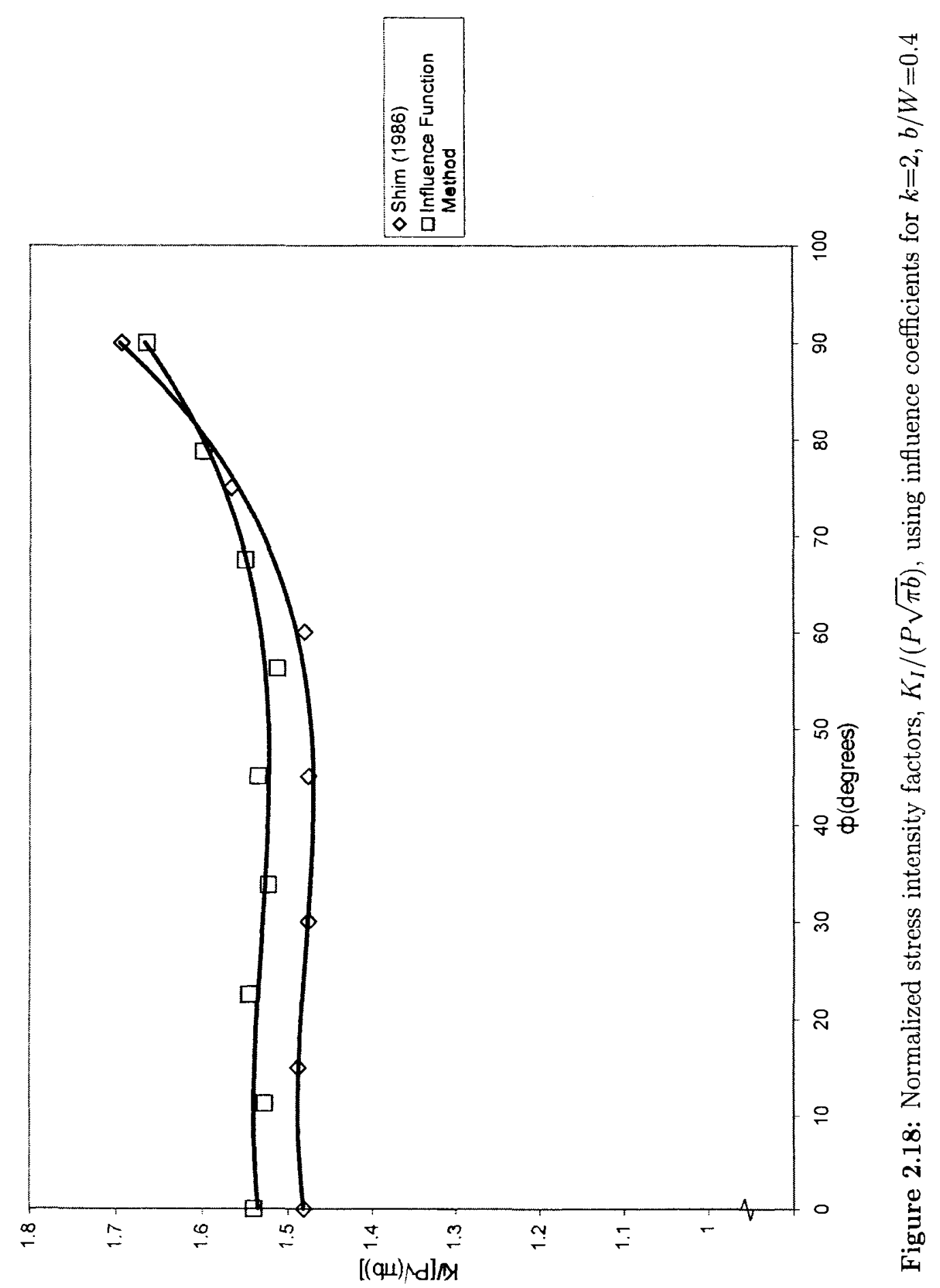




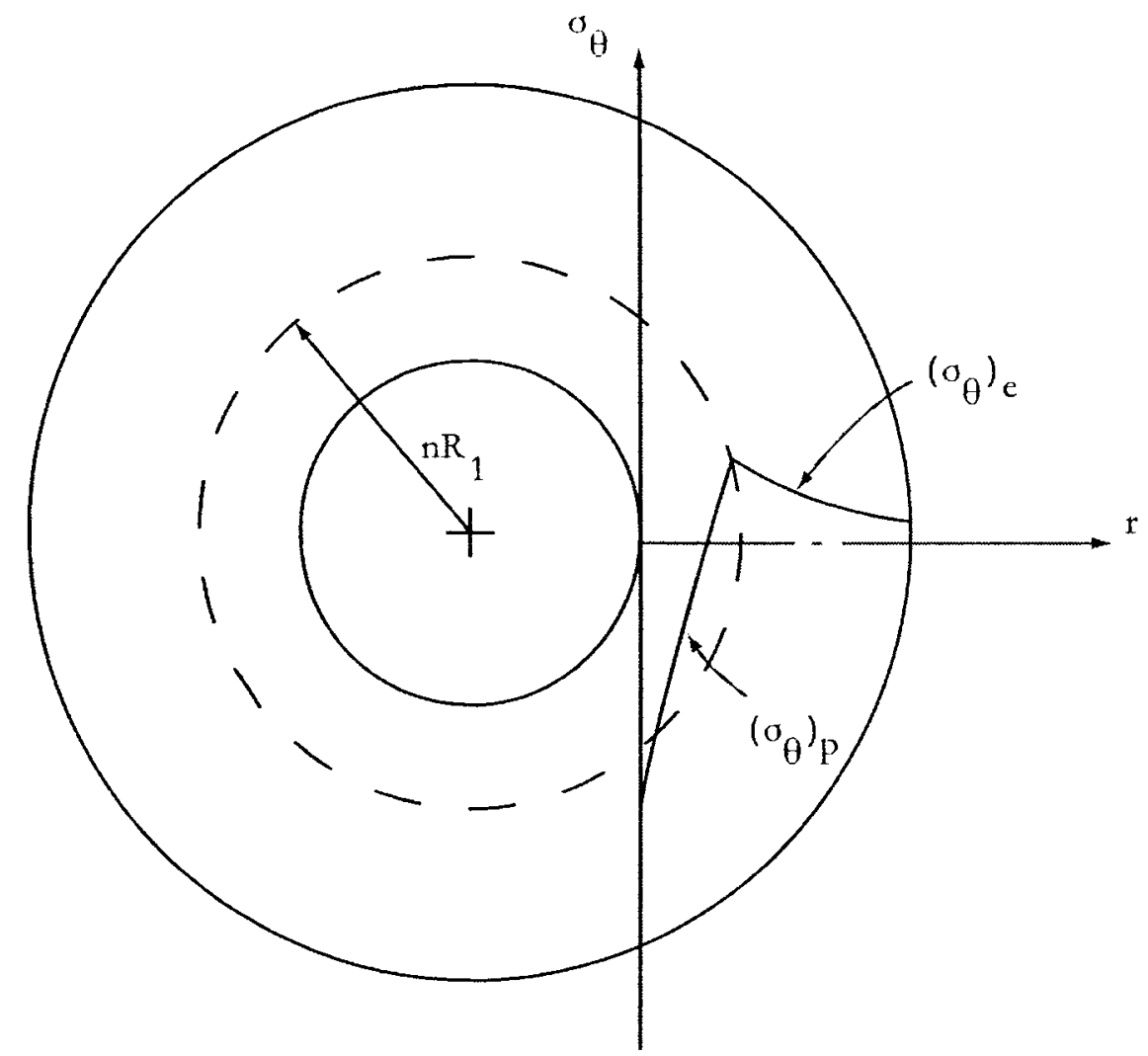

Figure 2.19: Typical residual hoop stress distribution in an autofrettaged cylinder 


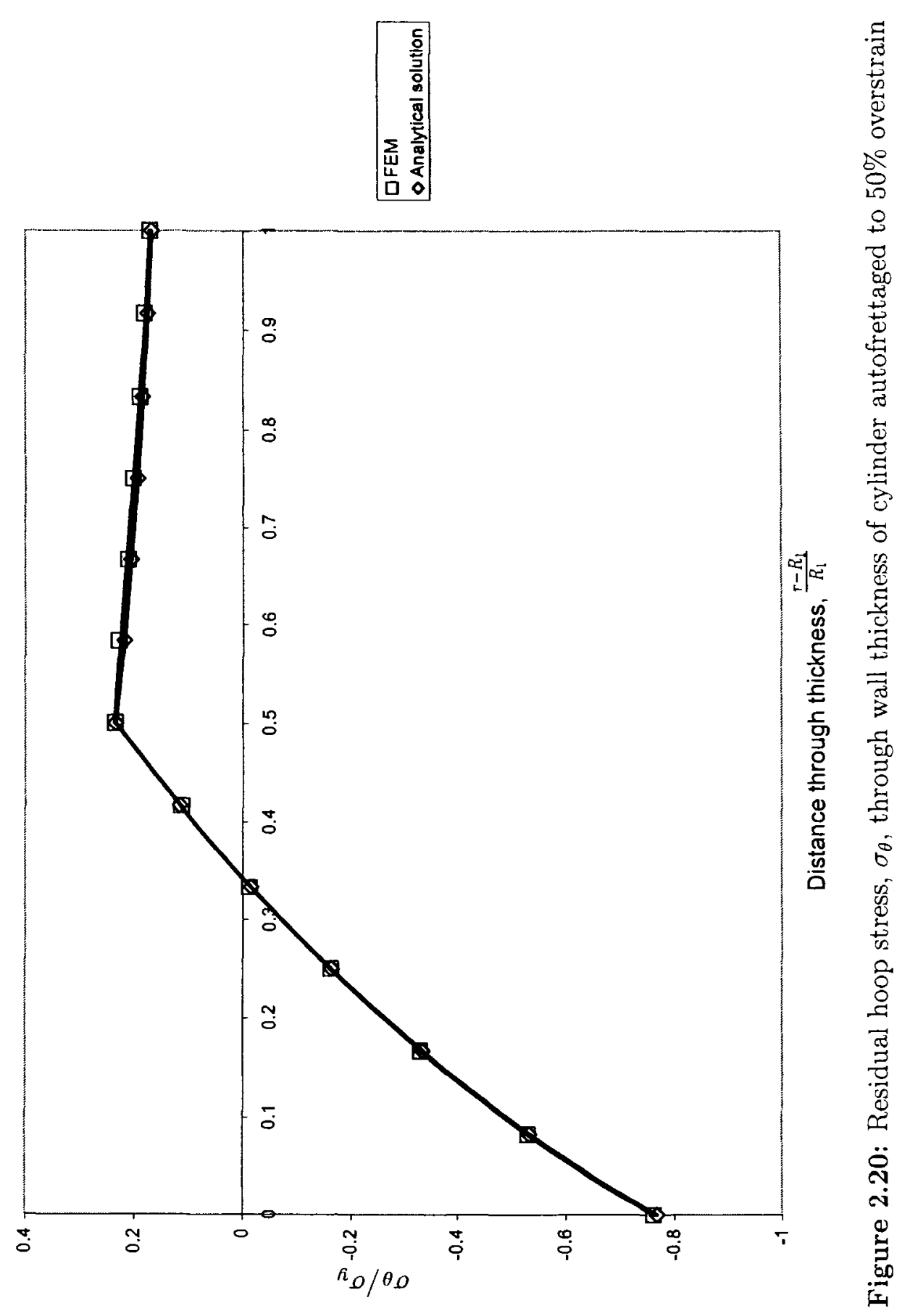




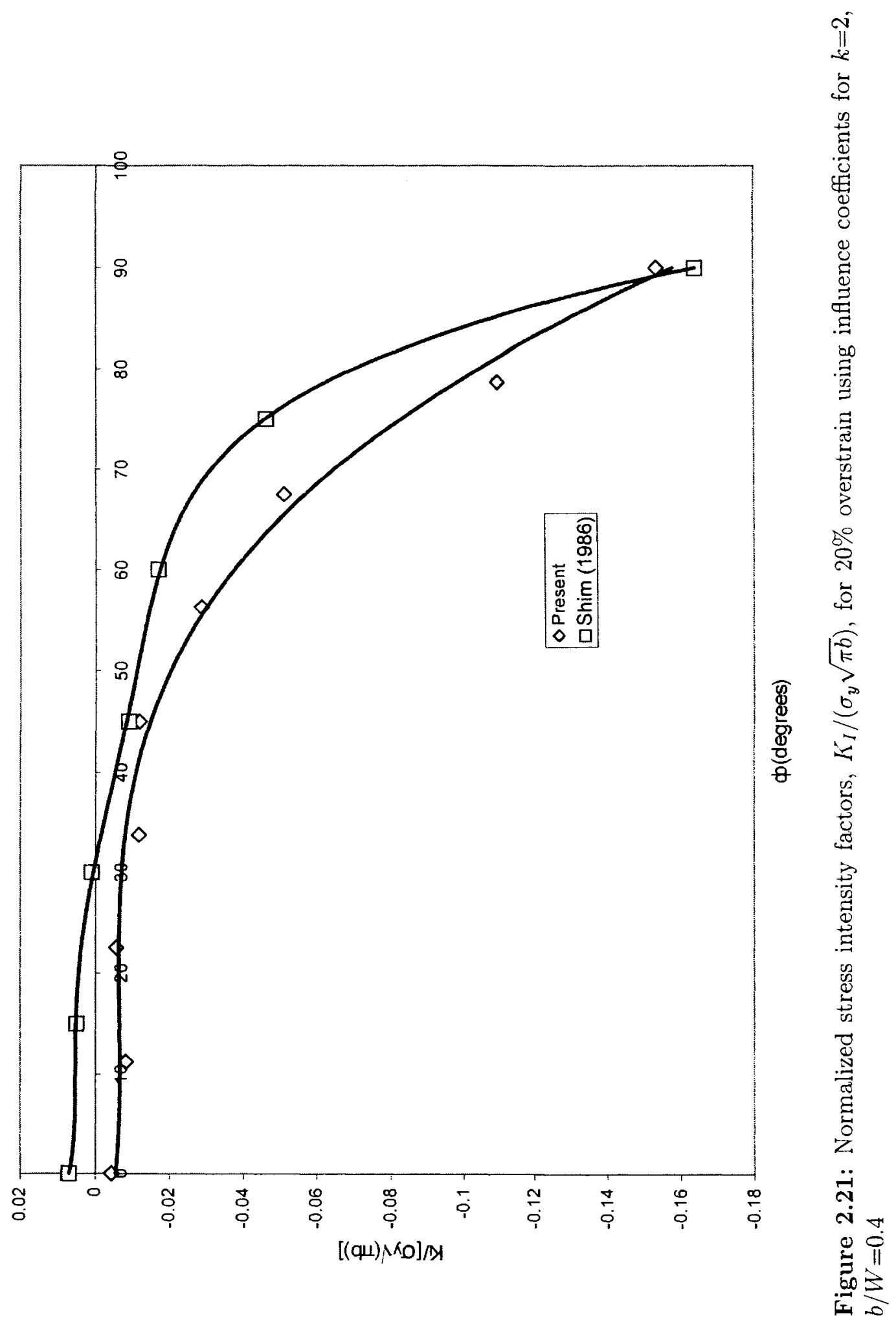




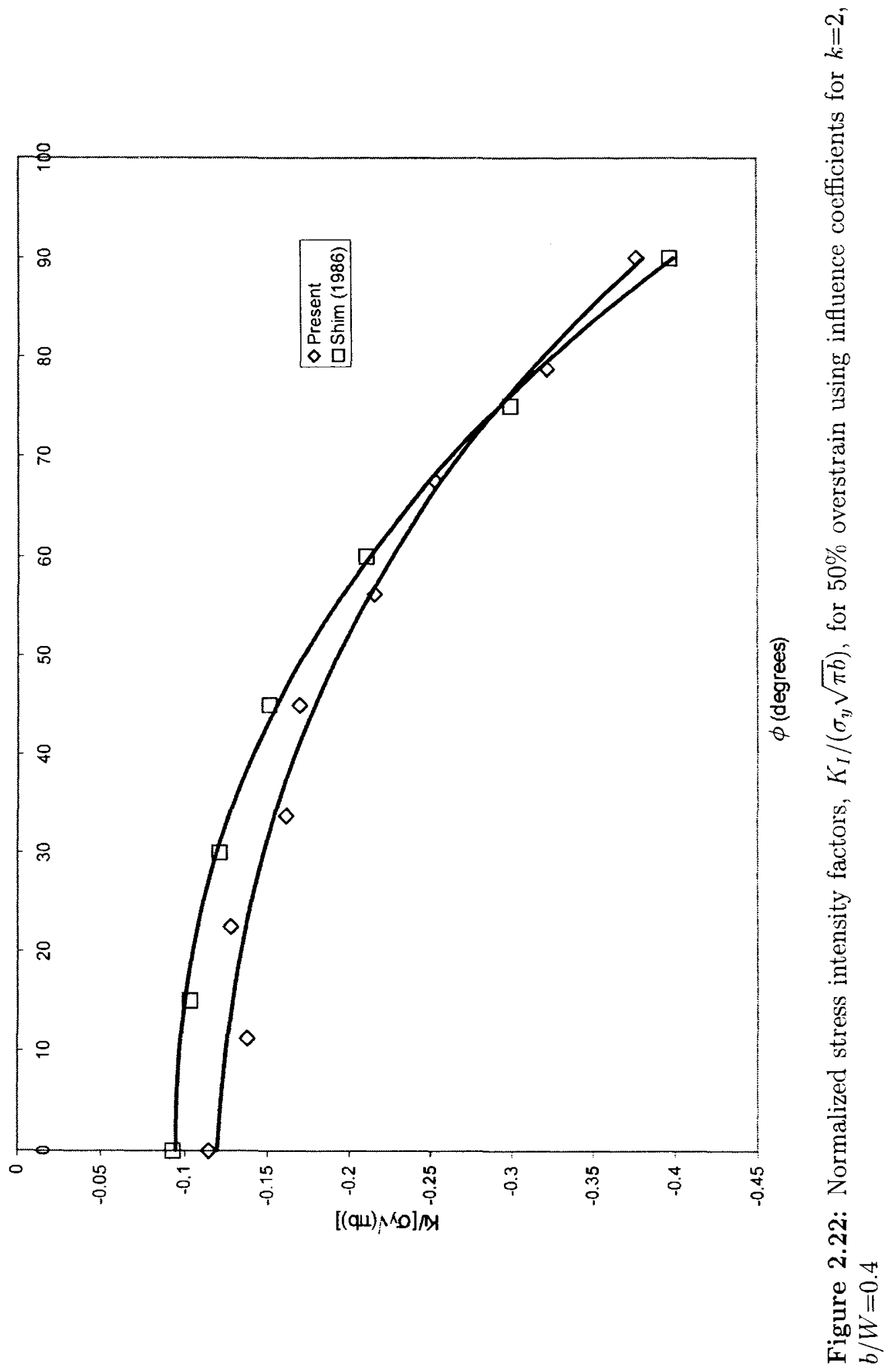




\section{Chapter 3}

\section{Stress Distributions in Pipe Bends}

The stress distribution in a thick-walled circular pipe bend under internal pressure, $P$, was analyzed to determine the location of the maximum stress; this will most likely be the site for crack initiation. Throughout this analysis the curved tube was assumed to have a uniform circular cross-section and be free of localized wall thinning, or any other defect, that may be imparted during the forming process. Figure 3.1 shows the geometry of the physical problem. Treating the circular pipe bend as part of a torus, it is convenient to introduce toroidal coordinate system $(r, \psi, \theta)$, as shown in the figure. In this figure, $R$ represents the radius of the pipe bend, $R_{1}$ and $R_{2}$ are the internal and external radii of the cross-section, $\psi$ is the circumferential angle and $\theta$ is the longitudinal angle. A total of 27 cases were analyzed from a combination of the following geometric parameters

$$
\begin{gathered}
R^{*}=R / R_{1}=5,7.5 \text { and } 10 \\
k=R_{2} / R_{1}=1.5,2.0 \text { and } 2.5
\end{gathered}
$$

and angular extent of $\theta$ for the pipe bend of

$$
\theta=45^{\circ}, 67.5^{\circ} \text { and } 90^{\circ} \text {. }
$$




\subsection{Numerical Model}

Taking advantage of symmetry, only a quarter of the physical problem needs to be modelled, as shown in Figure 3.2. An additional length of cylindrical tubing was added at the cessation of the bend curvature to simplify the application of boundary conditions to the numerical model. This tangential straight tube was $2 R_{2}$ in length. It was observed that the stress distribution in the straight tube were in agreement with the well-known Lamé solution for the thick-walled cylinder within this distance from the curvature.

The BEM mesh for each model has between 166 and 232 8-node quadrilateral elements. In all the models, vertical displacement constraints were applied along the horizontal plane of symmetry; also the cross-section created by the plane of symmetry at the mid-section of the bend was constrained to displace only in the radial direction. Plane strain end conditions were imposed at the end of the additional straight length of tube. The model was subjected to an internal pressure load, $P$.

\subsection{Numerical Results}

The hoop stress results obtained for the BEM analysis were normalized with respect to the corresponding Lamé solution, denoted as $\sigma_{o}$, for the cylinder of the equivalent $R_{2} / R_{1}$ ratio. As such, the values presented may be considered as stress factors, with a normalized value of unity corresponding to the hoop stress at the internal surface of a cylinder with identical radii dimensions and subjected to the same internal pressure.

The results for the normalized hoop stress obtained from the BEM analysis were also compared using a commercial code for finite element analysis, ABAQUS, for the same parameters and boundary conditions of the physical problem. It was necessary to use considerably more 20 -node brick elements and a much more refined mesh when 
using the FEM as opposed to the BEM. Figures 3.3 and 3.4 show there is good agreement of the results, $\sigma_{\psi} / \sigma_{o}$, between the FEM and BEM analyses. The $R^{*}=5$, $k=1.5, \theta=90^{\circ}$ case, shown in Figure 3.3, has the greatest discrepancy between the two numerical methods; however, it was less than $2 \%$ difference. In Figure 3.4, the $R^{*}=7.5, k=1.5, \theta=90^{\circ}$ case is more typical with a maximum difference of $1.1 \%$; many models remained well below $1 \%$ difference.

For $R^{*}=5, k=2, \theta=90^{\circ}$, the stress factor was plotted along both the intrados and extrados as shown in Figure 3.5. This result is consistent with the findings of Colle et al. (1987); the stress factor is greatest at the intrados and therefore this site is considered most of interest to be investigated further.

Figures 3.6 to 3.8 show the variation of the stress factor at the intrados along the circumferential direction of the pipe bend for each $k$ ratio investigated when $\theta=90^{\circ}$ and for the different values of $R^{*}$. It can be seen that the stress distribution increases nonlinearly with decreasing wall thickness. There is less difference between the $k=1.5$ and $k=2$ trend lines than between the $k=2$ and $k=2.5$. This is consistent with the stress state in a thick-walled cylinder which is inversely proportional to the difference of squares of the internal and external radii.

The effects of the parameter $R^{*}$ on the stress factor are shown in Figures 3.9 to 3.11 for the $k$ ratios analyzed. It is shown that the hoop stress is larger at a given $\theta$ position with smaller bend radius ratios, $R^{*}$. From these figures it can also be observed that increasing radius ratio, $k$, has little effect on $R^{*}=5$ and that the significance of the bend radius to $k$ ratio relationship is more apparent for the larger bend radii.

The stress increases throughout the curvature adjacent to the tangential straight pipe but then becomes relatively constant with respect to the longitudinal angle for the majority of the curvature. This is most evident in the $90^{\circ}$ plots, and more pronounced for larger values of $R^{*}$. Despite this trend, the stress remains below the 
axisymmetric solution present in a torus, as shown in Figure 3.12 which plots the case of $R^{*}=5, k=2$ over each value of $\theta$.

The normalized hoop stress increases with smaller bend radius and similarly for larger values of curvature, $\theta$, as evident from Figures 3.12 to 3.14 which show the effect of increasing $\theta$ for a constant radius ratio, $k=2$ at each value of $R^{*}$. Thus the stress distribution increases as the model becomes farther removed from a straight cylinder as one would expect. The largest stress factor occurs in the $R^{*}=5, k=1.5$, $\theta=90^{\circ}$ model and the smallest in the $R^{*}=10, k=2.5, \theta=45^{\circ}$ model.

\subsection{Conclusions}

The maximum circumferential stress in a curved tube under internal pressure occurs at the intrados. For a given radius ratio $k$, the circumferential stress factor $\sigma_{\psi} / \sigma_{o}$, has been found to increase with increasing curvature of the tube bend, i.e. with decreasing values of $R^{*}$. Larger stresses have also been observed with decreasing radius ratio $k$, but this was to be expected as it is consistent with the Lamé relation for a simple straight circular cylinder. Regardless of the combination of the geometric parameters, the intrados of the pipe bend is thus the most likely site for crack nucleation. 


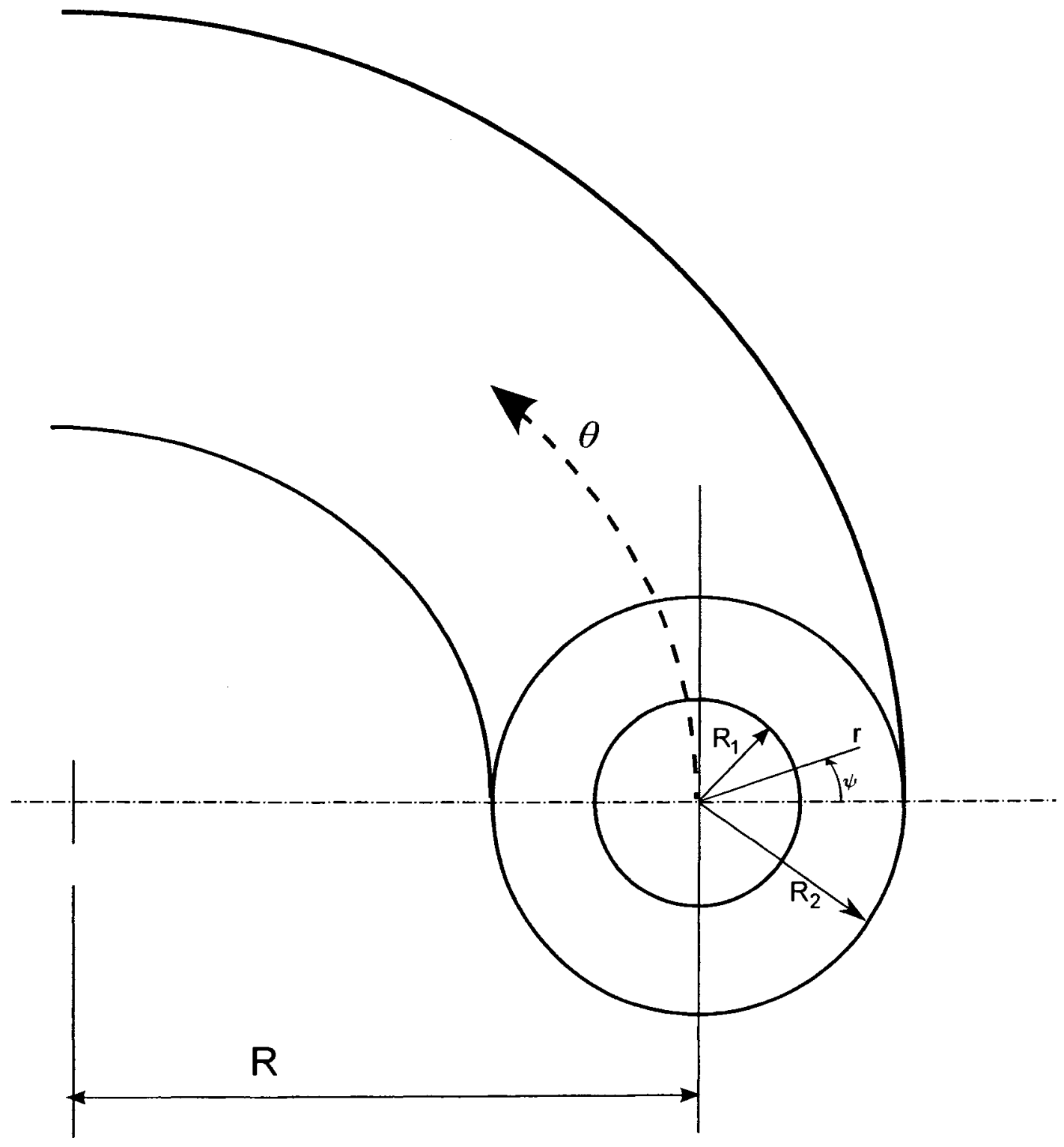

Figure 3.1: Curved tubing diagram 


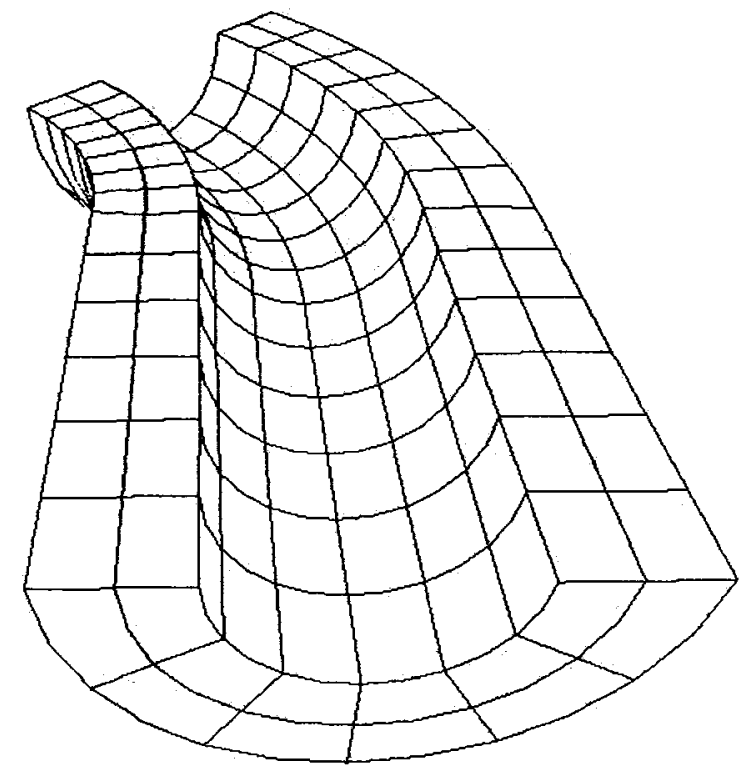

(a) BEM mesh with 232 elements, 698 nodes

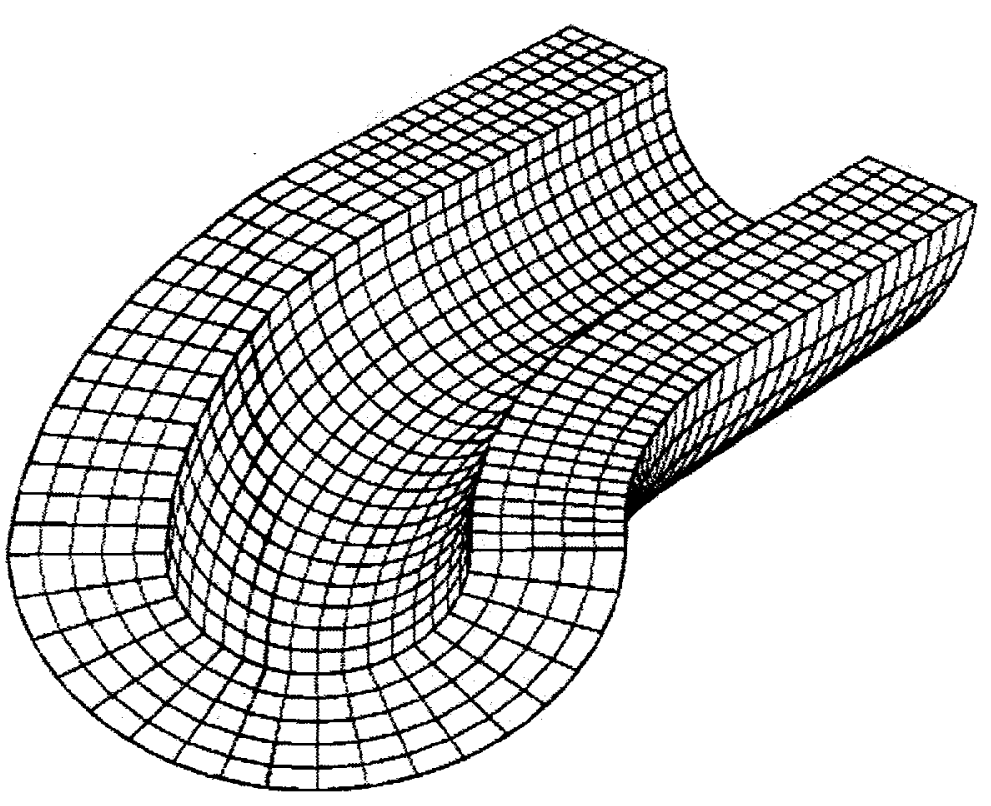

(b) FEM mesh with 2880 elements, 14109 nodes

Figure 3.2: Quarter-model mesh for $R^{*}=5, k=2, \theta=90^{\circ}$ 


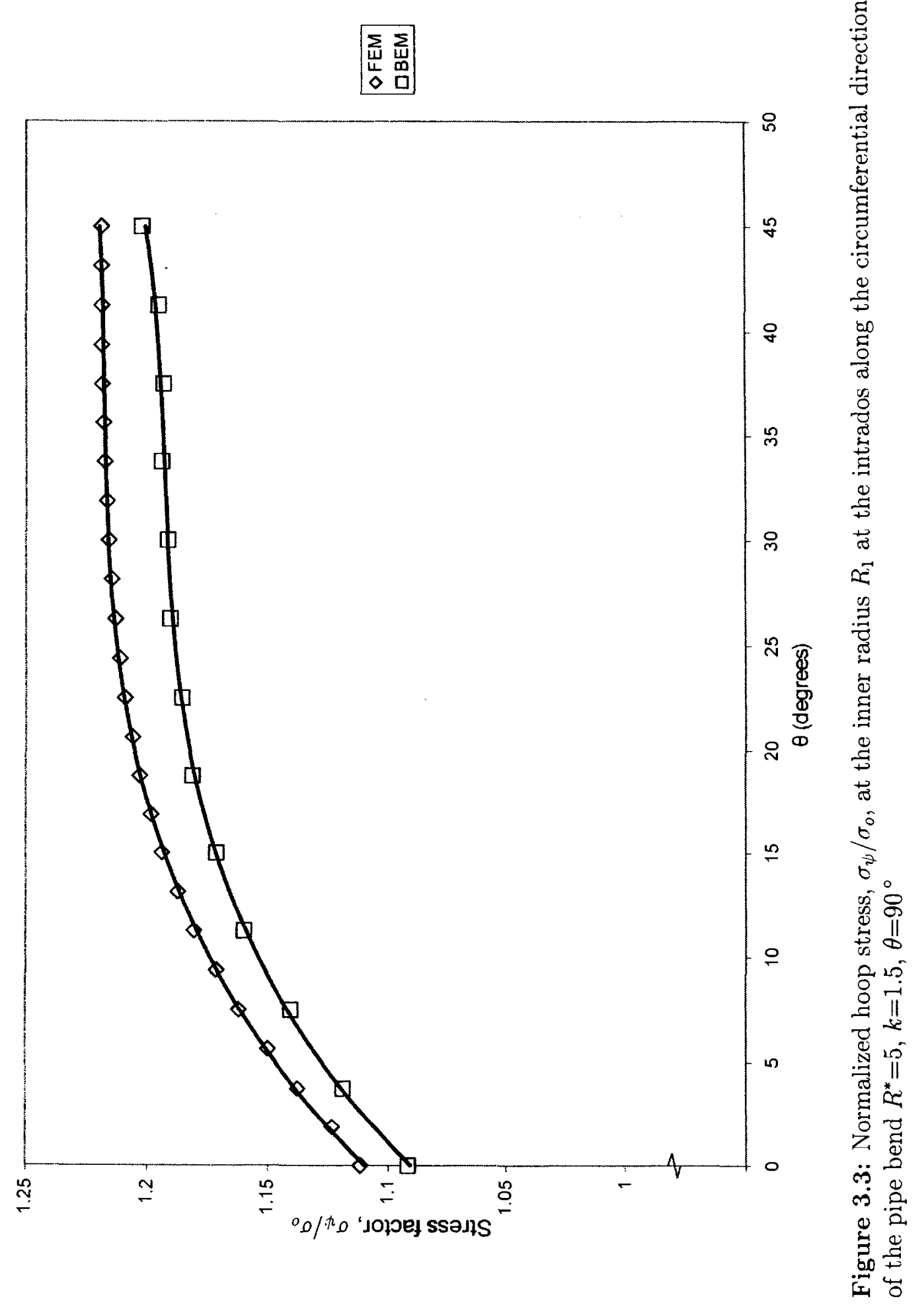




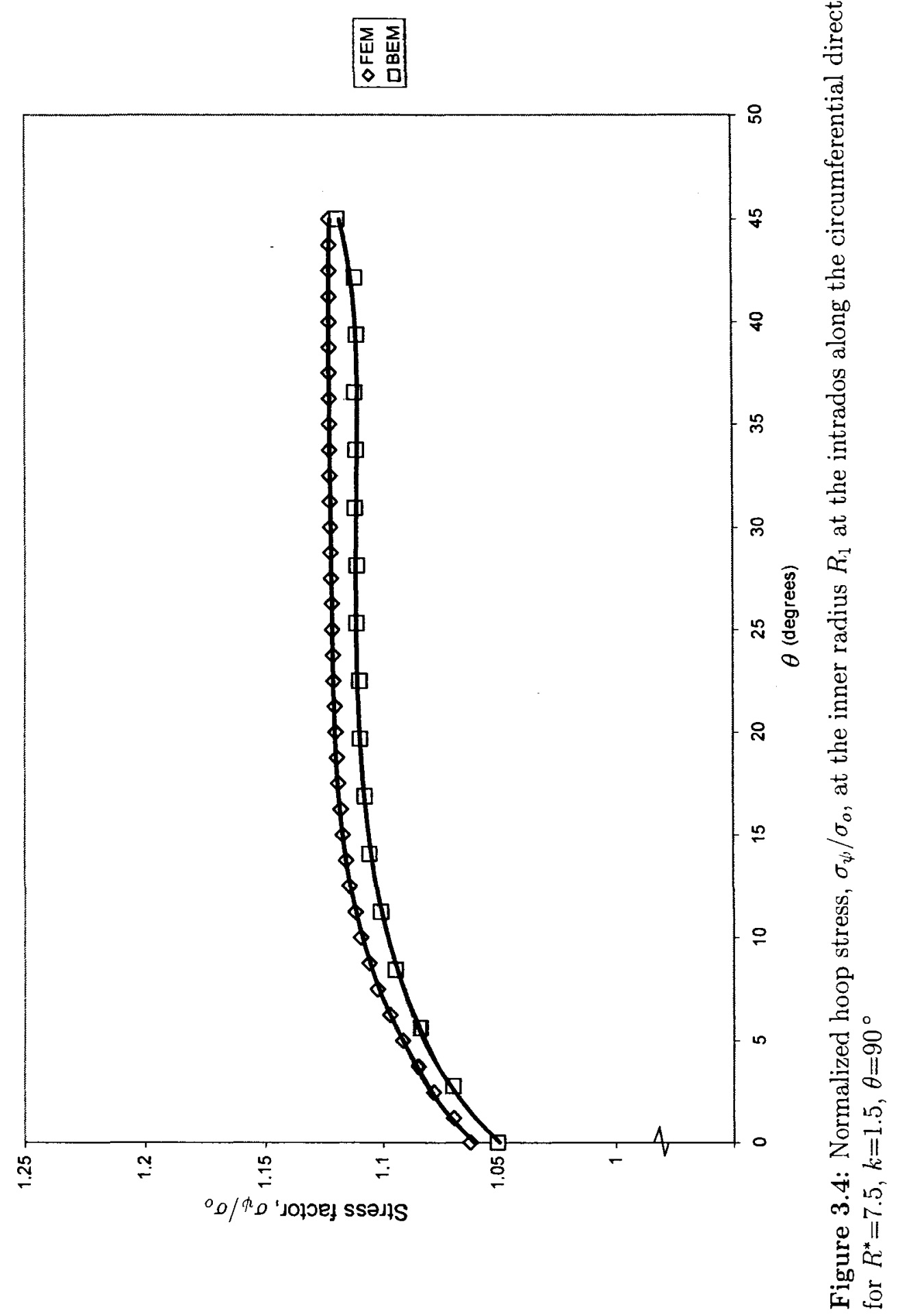




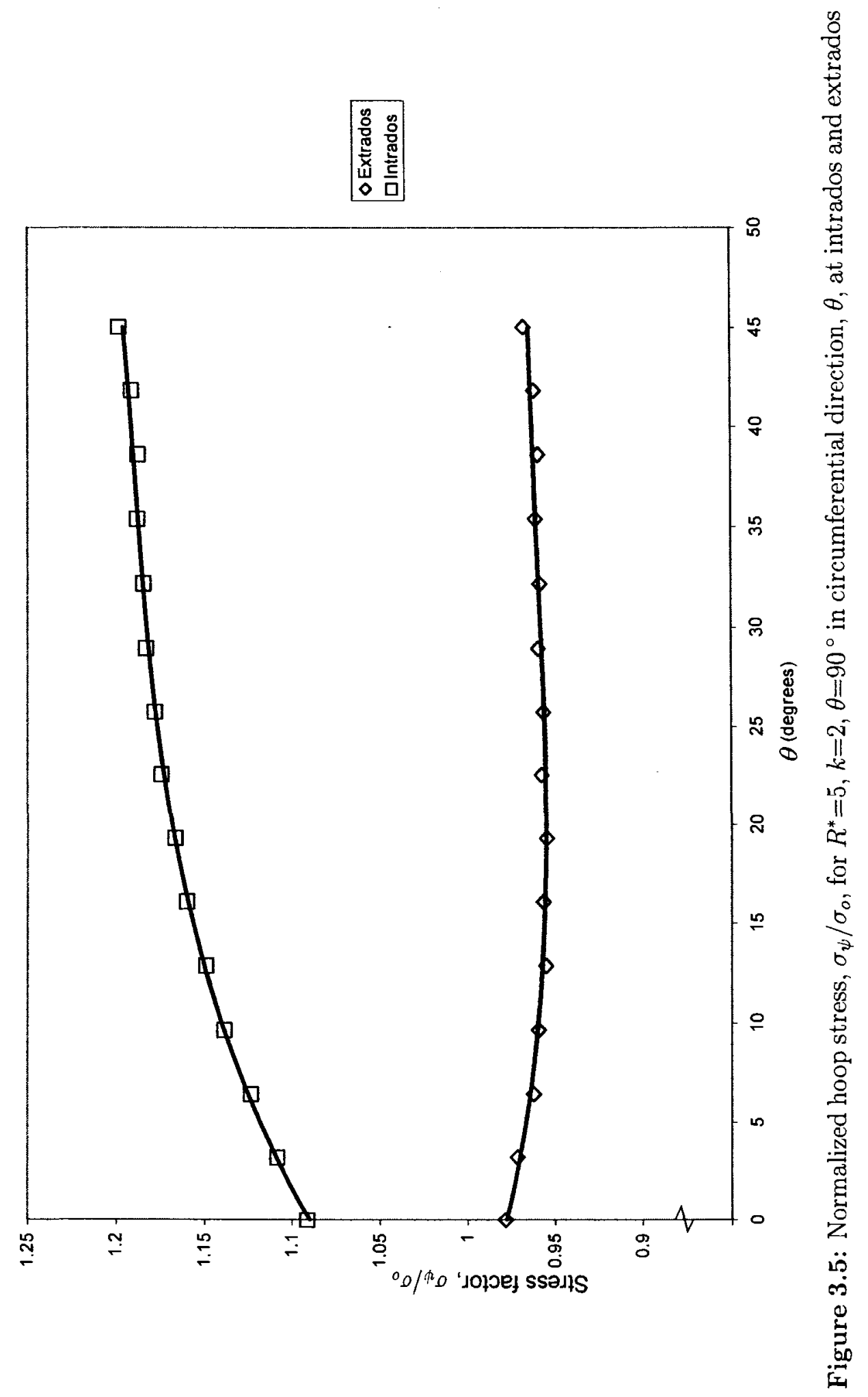




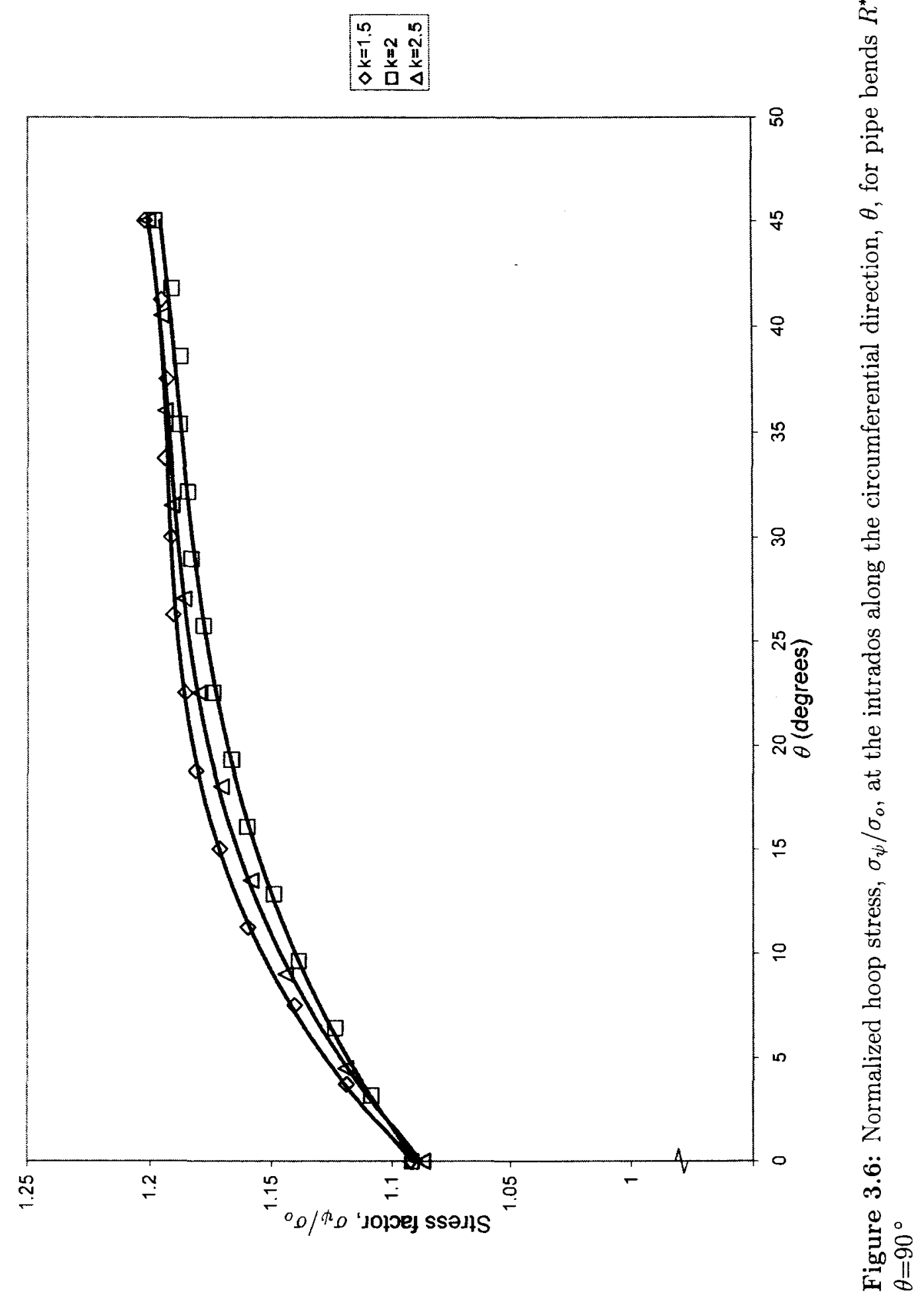




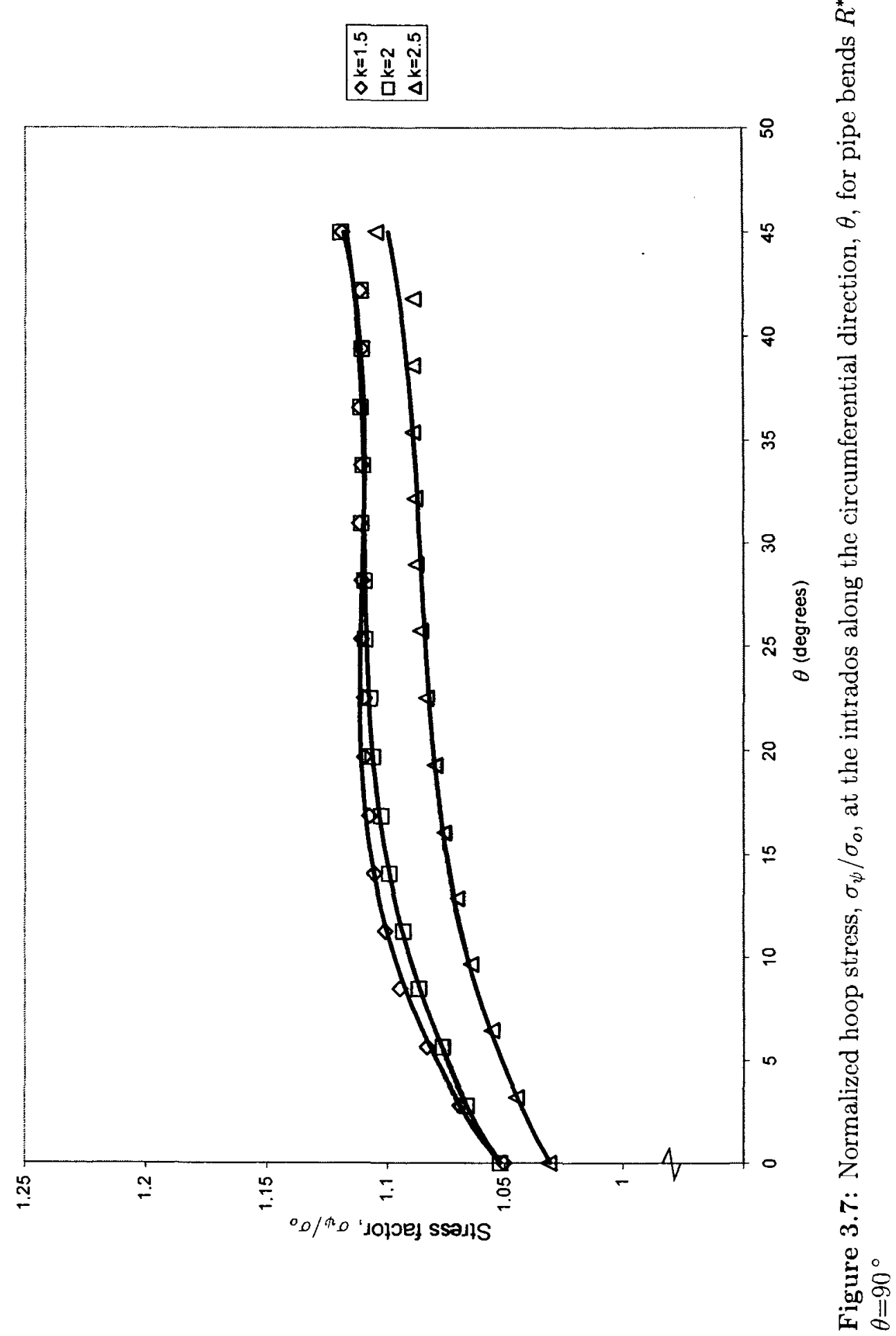




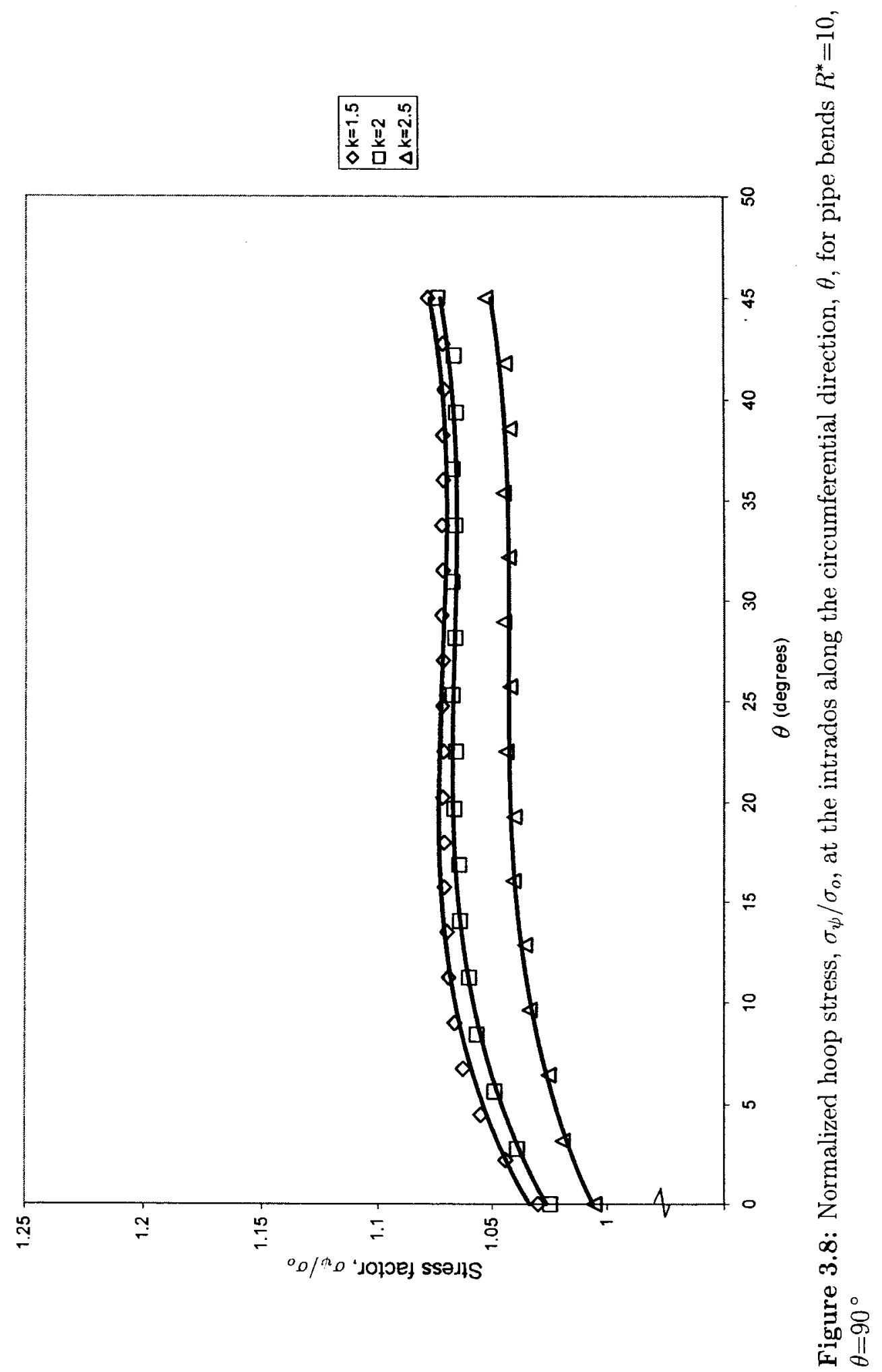




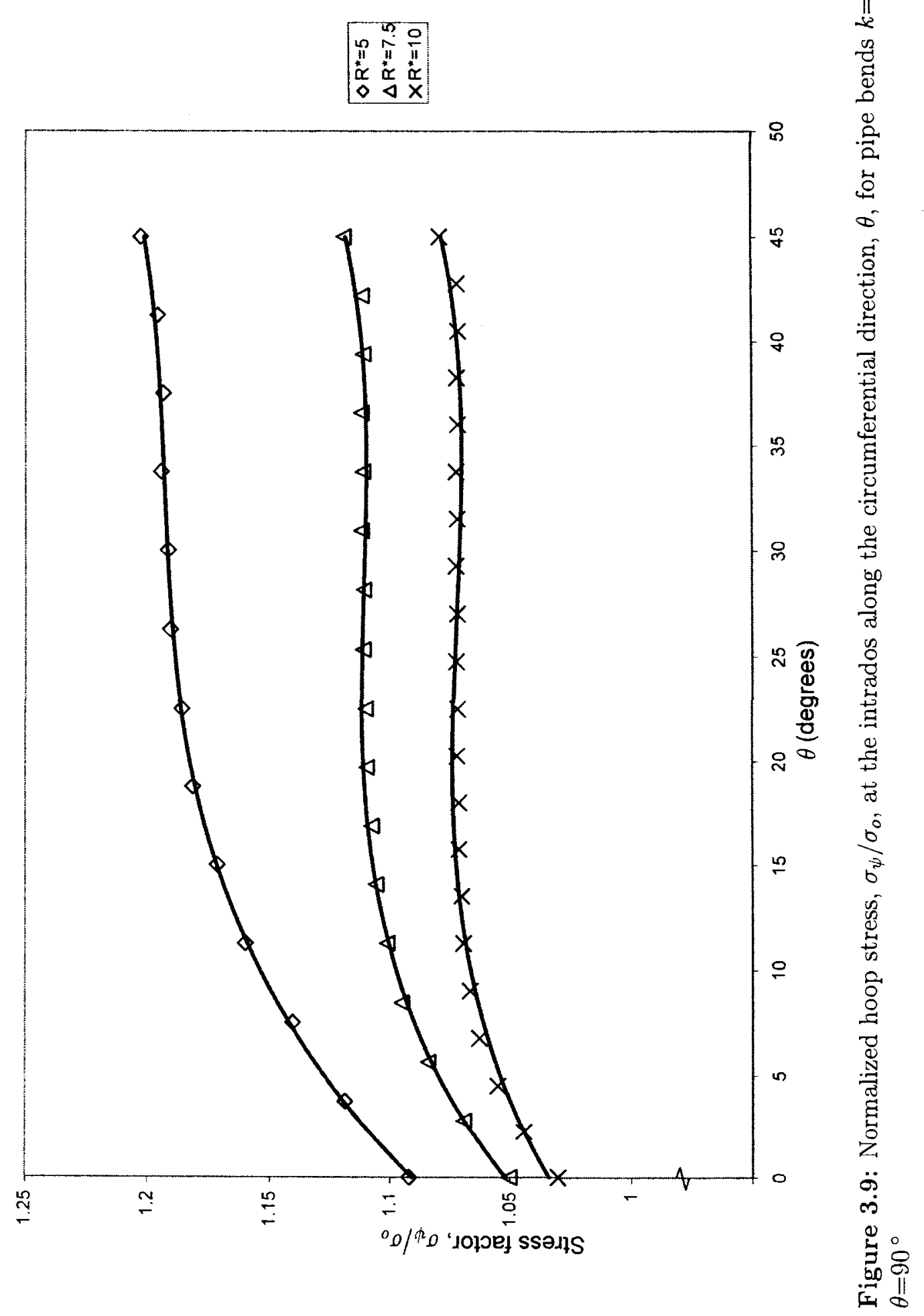




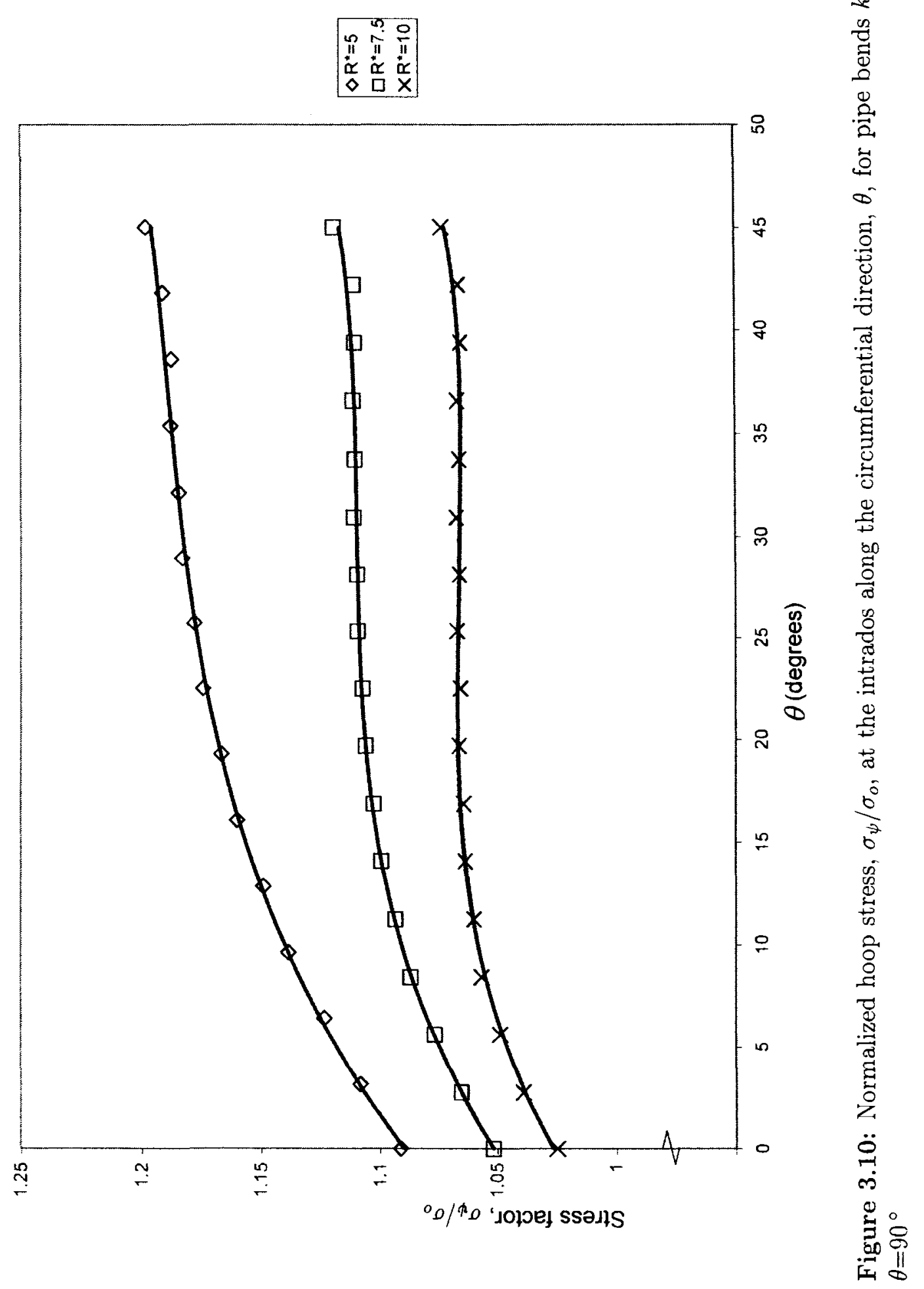




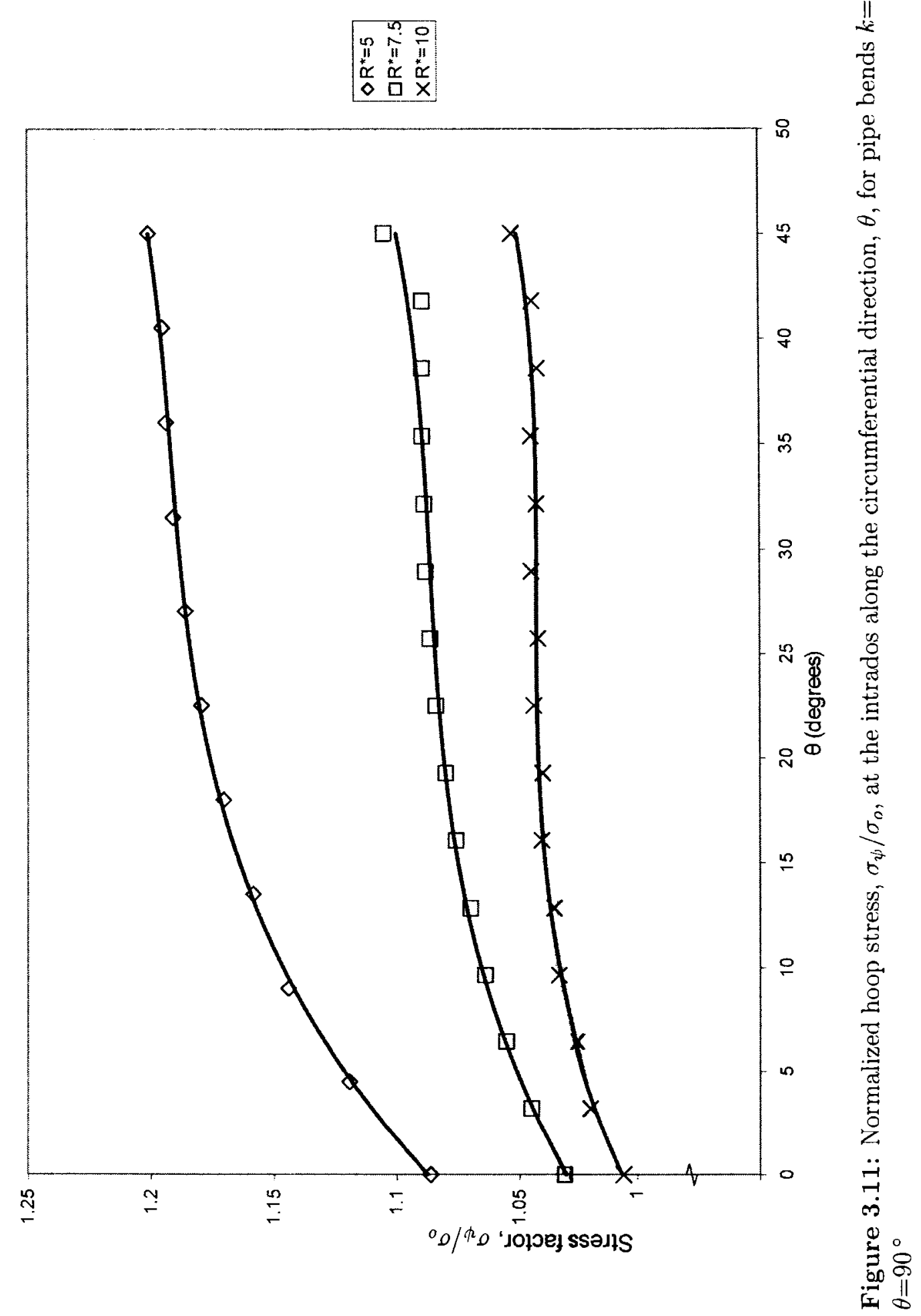




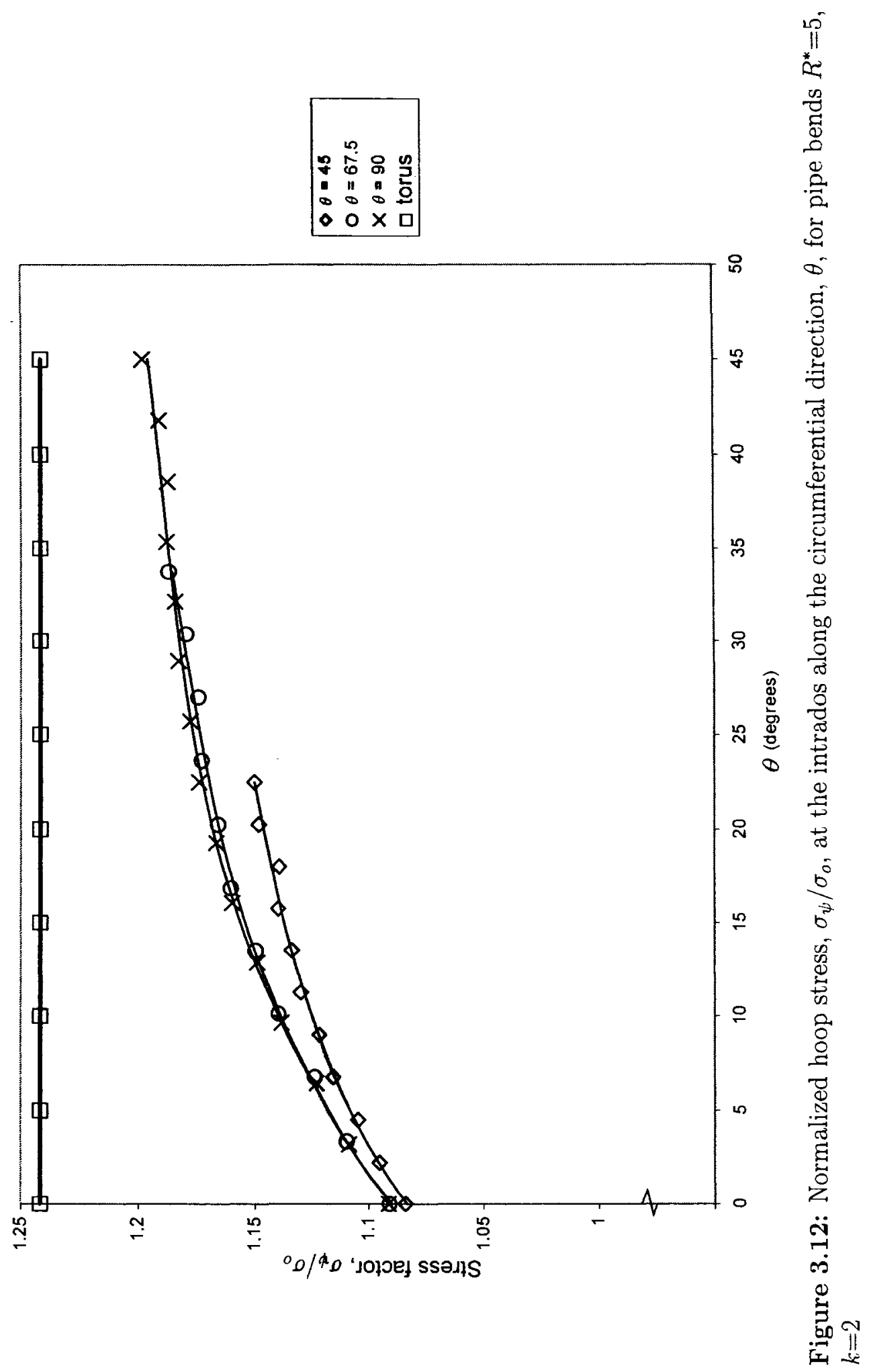




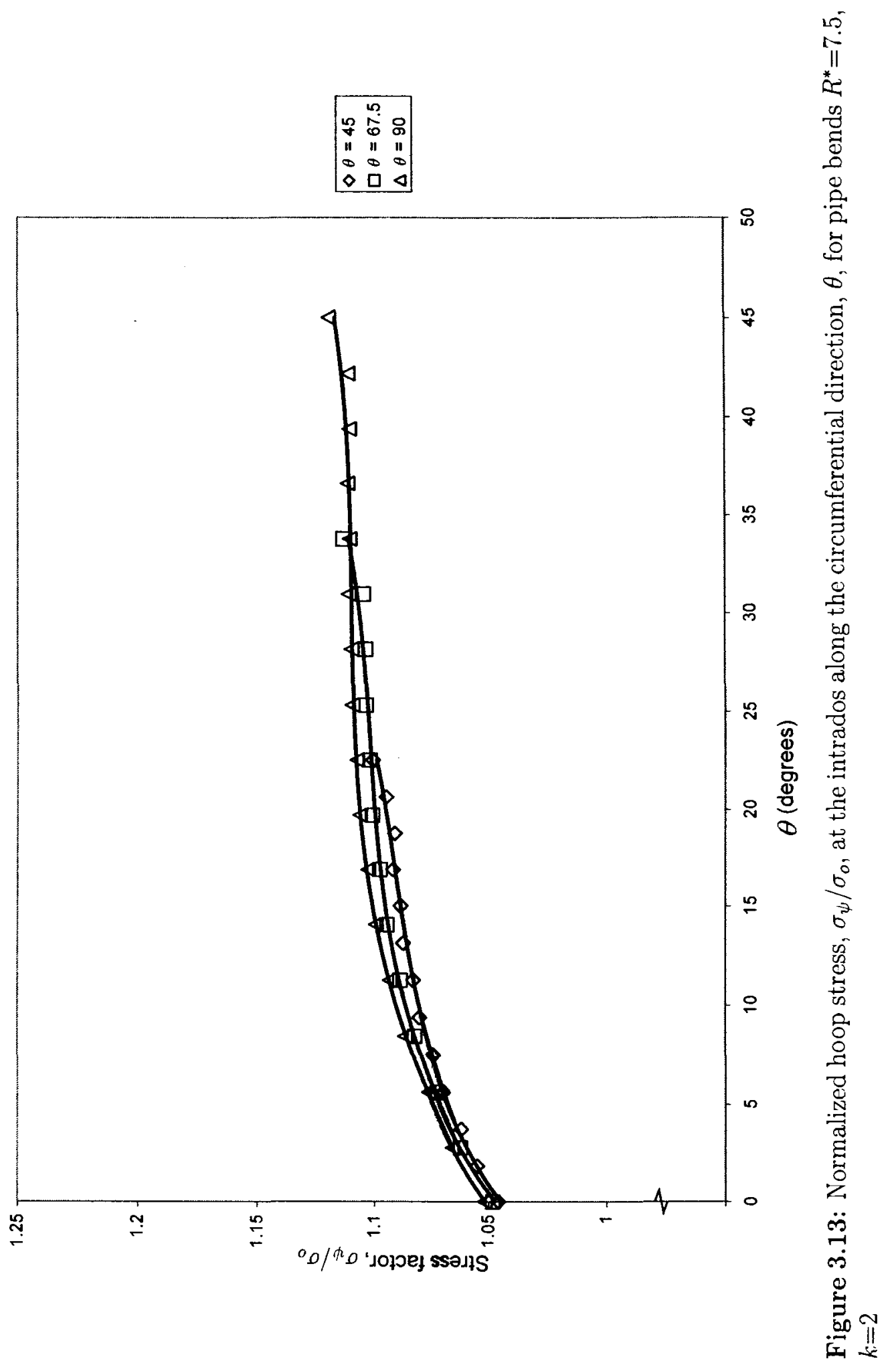




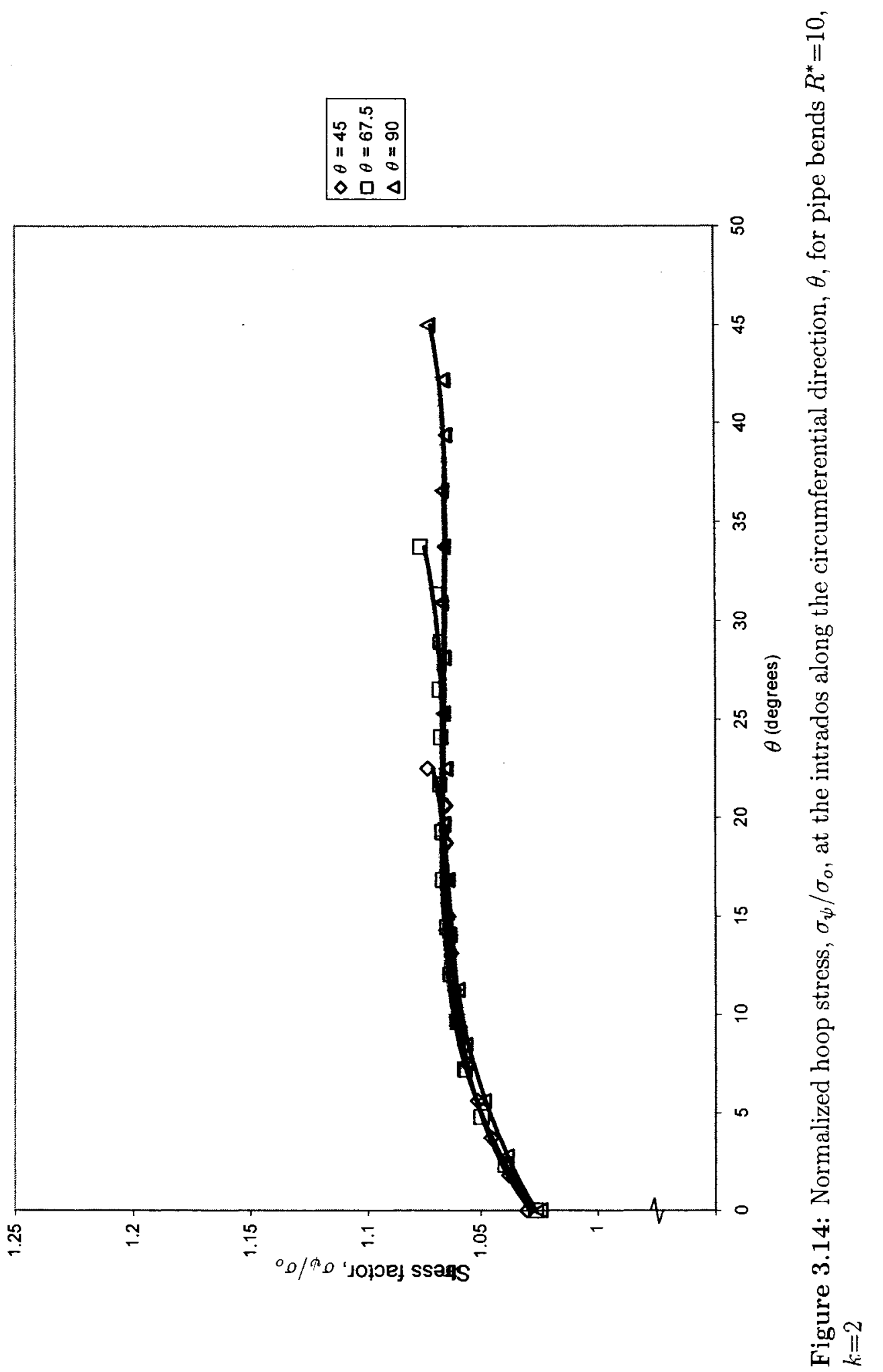




\section{Chapter 4}

\section{Stress Intensity Factors for a Crack in a}

\section{Pipe Bend}

In the previous chapter, it was established that the maximum hoop stress in the cross-section of a thick-walled pipe bend occurs at its intrados. This location is likely to be the site of crack initiation. In this chapter, a semi-elliptical crack is introduced there in the internally pressurized pipe bend, and stress intensity factors for a range of geometric parameters are obtained. Polynomial influence coefficients are also calculated and they are employed to obtain the stress intensity factors due to the residual stresses for various percentages of overstrain due to autofrettage.

\subsection{Semi-Elliptical Crack In a Pipe Bend}

Figure 4.1 shows a thick-walled pipe bend with a semi-elliptical crack at the intrados. In the present study, a semi-elliptical crack with an aspect ratio $b / a=0.8$ was investigated for each of the 27 geometric cases of the pipe bend analyzed in the previous chapter, namely

$$
\begin{gathered}
R^{*}=R / R_{1}=5,7.5,10 \\
k=R_{2} / R_{1}=1.5,2.0,2.5
\end{gathered}
$$




$$
\theta=45^{\circ}, 67.5^{\circ}, 90^{\circ}
$$

In each of these cases, four different relative crack depths, except one situation, are considered, namely

$$
b / W=0.2,0.4,0.6,0.8
$$

Stress intensity factors were obtained at nodal points lying along the crack-front for the cracked geometries treated. The angular positions of these points on the crackfront are defined by $\phi$, as shown in Figure 4.1. The one geometric case which was not modelled is that of $R^{*}=5, k=2.5, \theta=45^{\circ}, b / W=0.8$ where the crack would extend almost the full span in the circumferential $(\theta)$ direction of the tubing, and the size of the crack makes it unlikely to be a stable cracked geometry in practice. The choice of one semi-elliptical crack aspect ratio of $b / a=0.8$ being considered was drawn largely from the experience of straight cylindrical tubings (see, e.g. Tan \& Fenner (1980)); the main aim of the present study being to investigate the influence of the various geometric parameters for the curved tubing on the stress intensity factor for a given crack size.

\subsubsection{Modeling Considerations}

For every geometry that was modelled, four elements were employed along the crack face. The node placement at the free surface and its adjacent midpoint node do not have constant values of $\phi$, however. This is due to the curvature of the tube foreshortening the crack before it reaches a full $90^{\circ}$, unlike in a straight circular cylinder. The exact angle at the free surface and the midpoint were calculated and employed in modelling as shown in Table 4.1, but will be set nominally to $90^{\circ}$ and $78.75^{\circ}$ respectively in the tabulated results in what follows. These adjusted values of $\phi$ were found to be independent of $\theta$.

In each case, only a quarter of the physical problem was modelled due to symmetry. 
The number of triangular and quadrilateral quadratic elements in the BEM mesh which were employed varied with the complexity of the problem, from 100 elements and 288 nodes for $R^{*}=7.5, k=2.5, \theta=45^{\circ}, b / W=0.8$ to 175 elements and 501 nodes for $R^{*}=5, k=1.5, \theta=90^{\circ}, b / W=0.2$. Internal pressure, $P$, was applied to all elements on the internal surface of the tubing as well as the crack faces. A typical BEM mesh employed is shown in Figure 4.2.

In BEM modelling, it is allowable to employ elements with an aspect ratio of 4 or 5 and neighbouring elements to increase in size by 2 or 3 times. Thus, what may appear (when seen from the perspective of a FEM analysis) as a very coarse mesh is often sufficient. For this application, small elements were used to model the crack-front region and across the crack faces but the elements were gradually increased in size to match the coarser and more uniformly shaped elements elsewhere. As the stress distribution in a thick-walled component changes quite rapidly, there was concern over whether a single element across the wall thickness, far removed from the crack, would affect the accuracy of the stress intensity factor results. To this end, a convergence study to ascertain this was therefore conducted.

\subsubsection{Mesh Refinement Study}

The boundary element method is less mesh sensitive than the finite element method. However, it is still necessary to be mindful of the gradation between elements. The mesh for many of the models used throughout this work employed only one element across the wall thickness. Normally, this would be insufficient to accurately capture the stress distribution. To ensure that a converged solution for the stress intensity factor was being obtained even with a relatively coarse mesh discretization, though far removed from the crack-front, a mesh refinement convergence study was conducted. This consisted of a few models being discretized with multiple elements across the thickness and those results compared to the stress intensity factors obtained from the 
original mesh. For this purpose two arbitrarily chosen cracked geometries were analyzed: $R^{*}=7.5, k=2, \theta=90^{\circ}, b / W=0.4 ;$ and $R^{*}=7.5, k=2.5, \theta=90^{\circ}, b / W=0.6$. The BEM meshes for the former geometric case are shown in Figure 4.3. The comparison of the results are shown in Table 4.2. It can be seen that the addition of an extra boundary element across the wall of the tubing did not affect the computed stress intensity factor in any significant way at the all points along the crack-front for the two cracked geometries analyzed; the differences being less than $0.4 \%$.

\subsubsection{Results}

Due to the absence of a theoretical solution for this problem, the veracity of the results were assessed by checking the values of the stress intensity factors obtained from the displacement formula against the corresponding values obtained by the traction formula. Reasonable agreement of these values would suggest that the mesh design employed was suitable and adequate, as has been demonstrated in Chapter 2. Also, due to the voluminous amount of result data from the 107 individual cases that were analyzed in the fracture mechanics study, only some sample sets of these results will be presented in this Chapter. They represent the general trends observed in all the cases treated. The complete sets of the results obtained are compiled in Appendix B.

Table 4.3 shows the correlation between the traction and displacement formulas for $R^{*}=5, k=2.5, \theta=90^{\circ}, b / W=0.2,0.4,0.6$ and 0.8 . The results shown in Table 4.3 for $b / W=0.8$ represent the poorest agreement between formulas for any combination of parameters investigated; the results for $b / W=0.2$ were more typical. It is evident that there was less agreement between the values determined using the midpoint displacement formula as the data used was a greater distance from the crack face as discussed in Section 2.2.1. In general, with the exception of the free surface and adjacent midside node, the agreement was within $5 \%$.

The $1 / \sqrt{r}$ relation of the traction formula does not hold in close proximity to the 
free surface and this is observed in the results obtained. However, the traction formula is conventionally considered more accurate than the displacement formula as it is less mesh sensitive. Both the traction and displacement formula results were calculated at every node on the crack periphery for every case and found to be in excellent agreement, with the exception of the node at the free surface. The displacement formula was used throughout unless otherwise indicated.

The results listed in Appendix B are in the form of Table 4.4 which gives the normalized stress intensity factors, $K_{I} /(P \sqrt{\pi b})$, along the crack periphery for $R^{*}=5$, $k=2$. Interestingly, the normalized stress intensity factors are sufficiently consistent to be considered constant along most of the crack-front. Approaching the free surface however, typically beyond $\phi=60^{\circ}$, the stress intensity factor increases rapidly, as expected.

Figures 4.4 to 4.6 show the normalized stress intensity factors observed along the crack periphery, $\phi$, for values of $\theta$ at each radius ratio, $k$, for $R^{*}=5, b / W=0.6$; a larger value of $\theta$ is shown to result in a larger stress intensity factor for lower radius ratios. There is a much larger difference between the $k=2$ and 2.5 trend lines than the $k=1.5$ and 2 trend lines. This is consistent with the stress factor results presented for an uncracked curved tubing in Section 3.2.

Figures 4.6 to 4.8 each show the normalized stress intensity factor along the crack periphery for a value of $R^{*}$ and all values of $\theta$ with $k=2.5, b / W=0.6$. Increasing the bend radius ratio results in a decrease of the normalized stress intensity factor which is greater from $R^{*}=5$ to 7.5 than from $R^{*}=7.5$ to 10 . This is also consistent with earlier findings for stress factors. These figures also indicate a decreasing relevance of the circumferential extent of the tube bend, $\theta$, with increasing $R^{*}$. In the $R^{*}=10, k=2.5$, $b / W=0.6$ plot, Figure 4.8 , the points defining each gradation of $\theta, 45,67.5$ and $90^{\circ}$, are indiscernable. Thus, the results are almost entirely independent of the angular span of the bend. It is evident that the effect of $\theta$ is minimal and the normalized 
stress intensity factor corresponding to $\theta=90^{\circ}$ would provide a reasonable estimate for smaller angles.

Figure 4.9 shows a plot of the normalized stress intensity factors for each crack depth when $R^{*}=5, k=2, \theta=90^{\circ}$. It can be seen that the stress intensity factors along the crack profile are greatest at $b / W=0.2$ and 0.8 . Additionally, the stress intensity factors along the crack periphery increases more steadily with increasing crack depth; there is a notably steeper curve for $b / W=0.8$ as it approaches the free surface.

\subsection{Stress Intensity Factors from Polynomial In- fluence Coefficients}

Stress intensity factors can also be determined using polynomial influence coefficients as described in Section 2.4. This method allows for alternate load cases to be analyzed for a given cracked geometry using the superposition principle thus circumventing the need to repeat the BEM analysis in its entirety. In the following, the influence coefficients for a curved tubing with a semi-elliptical crack are determined and are used to verify the stress intensity factors presented above. As an application of the influence functions, the stress intensity factors due to the residual stresses resulting from autofrettage of the tubing are also obtained.

\subsubsection{Modelling Considerations}

The polynomial influence coefficients for a given cracked geometry were determined using the same mesh as for the direct boundary element method analysis. Four load cases were applied to each model; uniform $\left(\sigma=-A_{0}\right)$, linear $\left(\sigma=-A_{1} r_{s}\right)$, quadratic $\left(\sigma=-A_{2} r_{s}^{2}\right)$, and cubic $\left(\sigma=-A_{3} r_{s}^{3}\right)$ traction distributions were applied to the crack face and each normalized to reach a value of unity at the maximum crack depth. The 
$A_{i}$ coefficients obtained are provided in Tables $4.5,4.6$ and 4.7 for cases with $R^{*}=5$, 7.5 and 10 , respectively.

In defining the stress distribution as a third degree polynomial, the parameter $r_{s}$ was defined as indicated in Figure 4.1. The tractions applied to the crack face were normalized to allow $r_{s}$ to reach unity at a depth equivalent to $b$. This definition of $r_{s}$ would provide slightly higher stress intensity factors as opposed to if it had been defined in a rectangular coordinate system. The stress in the pipe bend decreases as $\theta$ moves away from the intrados and this definition of $r_{s}$ would hold the stress to be constant along the inner surface of the pipe bend for the duration of the crack face. However, the difference between the curvilinear and rectangular definitions of $r_{s}$ was determined to be minimal and the agreement between the influence coefficient method and the previous BEM was within $2.5 \%$ for both definitions.

Once the influence coefficients were determined they were combined with the stress distribution across the wall of the uncracked internally pressurized tubing to verify the stress intensity factors obtained directly from the BEM analyses as presented in the previous section above. This stress distribution was determined using the ABAQUS finite element software and was further approximated by least squares method to be a cubic polynomial. The use of the FEM was not absolutely necessary here and was employed merely for expediency because of the available preprocessing for mesh refinement required for more accurate (interior) solutions across the wall of the tubing at different positions of $\theta$. It was found that the resultant stress intensity factors were very sensitive to even small changes in the stress distribution.

\subsubsection{Verification}

The complete set of results for the influence coefficients obtained for the BEM analysis are listed in Appendix C; Table 4.8 shows a representative set for $R^{*}=5, k=2, \theta=90^{\circ}$. All these influence coefficients were then used to produce stress intensity factors 
for the corresponding internally pressured cracked pipe bend which were compared with those obtained directly by the BEM to establish the veracity of these influence coefficients. Results for the $R^{*}=5, k=2, \theta=90^{\circ}$ case are typical, the comparison of the results are shown in Table 4.9; the discrepancies were less than $2.5 \%$. The largest discrepancy between the normalized stress intensity factors obtained directly and those from the influence coefficient method was for the case of the pipe bend with $R^{*}=10, k=1.5, \theta=90^{\circ}$, and relative crack depth $b / W=0.2$, at just below $5 \%$ for the point at the free surface, as shown in Table 4.10. However, for the $R^{*}=10, k=1.5$, $\theta=90^{\circ}, b / W=0.8$ case shown in the same Table, there was excellent agreement, with less than $1 \%$ difference. This suggests that the mesh used in the model in the $b / W=0.2$ case can perhaps be improved upon, as the same uncracked stress distribution was applied to all of the crack depths for $R^{*}=10, k=1.5, \theta=90^{\circ}$. The discrepancies of the stress intensity factors for the $R^{*}=10, k=1.5, \theta=90^{\circ}, b / W=0.2$ case are, however, still relatively small in general to be of concern.

\subsubsection{Application: Effect of Residual Stresses from Autofret- tage}

Influence coefficients allow stress intensity factors for alternate load cases to be obtained using the superposition principle in a simple manner without the need to repeat the numerical analysis in its entirety for the same cracked geometry. To demonstrate this, the influence coefficients determined above were applied to determine the stress intensity factors arising from the residual stresses of pipe bends, with radius ratio $k=2.0$, which had undergone various degrees of overstrain from autofrettage. 


\section{Modelling Considerations}

In this aspect of the study, each of the tube bends was analyzed at $10 \%$ increments of overstrain from $10 \%$ to $100 \%$ in the autofrettaged process, before the onset of crack growth. The material was assumed to be elastic-perfectly plastic with a yield stress of $\sigma_{y}$ and that von Mises yield criterion with strain-history independent plastic deformation was applicable to this material.

ABAQUS was employed to determine the residual hoop stress in the uncracked pipe bend. This was necessary as the BEM software used was not capable of modelling plasticity effects. Due to the lack of a theoretical solution for the residual stress or required pressure to obtain a specified percent overstrain for this particular geometry, a trial and error process was undertaken. To minimize error throughout this procedure, each set of results was plotted to verify the trends of stresses were consistent with those expected and the pressure adjusted accordingly.

For those cases where the elastic-plastic interface in the wall of the circular crosssection, i.e. at $r=n R_{1}$ was equal to or greater than the crack depth, the residual hoop stress distribution in the curved tubing due to autofrettage was determined using ABAQUS; this was then used with the influence coefficients to obtain the stress intensity factors, as was done for the internal pressure case. When $n R_{1}$ was less than $b$, the influence coefficients were not employed as the stress distribution across the crack face could not be represented with a single cubic polynomial. Therefore, the stress distribution was obtained using ABAQUS and the appropriate tractions were applied to the crack face so that the stress intensity factors could be computed directly. 


\section{Results}

The complete sets of results of stress intensity factors due to the residual hoop stresses for different percentages of overstrain are available in Appendix $D$; all results shown are from the traction formula, as opposed to the displacement formula presented elsewhere. Normalized stress intensity factors at different percentages of overstrain are given in Tables 4.11 to 4.13 , for $k=2, \theta=90^{\circ}, b / W=0.6$ at $R^{*}=5,7.5$ and 10 . It can be observed that most of the normalized stress intensity factors are nearly constant in the elastic region, but steadily decrease towards the free surface.

Many of the normalized stress intensity factors, particularly for smaller crack depths, have negative values. These are not physically meaningful in terms of a stress intensity factor but indicate that the residual hoop stresses imparted due to autofrettage are compressive and thus any subsequent pressure load will have to overcome this before opening the crack further. These negative values were a consistent result across all crack depths in close proximity to the bore of the pipe bend but the stress intensity factor was not a constant along the crack periphery. In cases where the crack depth exceeded the elastic-plastic interface, the stress intensity factors were positive further removed from the bore and thus the residual stresses in the pipe were no longer beneficial in retarding crack growth.

\subsection{Conclusions}

Stress intensity factors were determined for a semi-elliptical crack of $b / a=0.8$ in an internally pressurized curved tubing. These were investigated for: three bend radii, $R^{*}=5,7.5,10 ;$ three ratios of outer to inner diameter, $k=1.5,2,2.5 ;$ three bend durations, $\theta=45^{\circ}, 67.5^{\circ}, 90^{\circ}$; and four crack depths, $b / W=0.2,0.4,0.6,0.8$. The only combination of parameters omitted was $R^{*}=5, k=2.5, \theta=90^{\circ}, b / W=0.8$. The stress intensity factors were observed to be constant along most of the crack periphery 
and then increased rapidly as the free surface was approached. The rate at which the stress intensity factor increased as it approached the bore was more pronounced with increasing crack depth.

The circumferential extent of the entire tubing, as denoted by the parameter $\theta$, was determined to have negligible influence on the stress intensity factors, particularly with increasing bend radius, although larger values of $\theta$ resulted in slightly higher $K_{I}$ values. Increasing the radius ratio or the bend radii both resulted in decreasing stress intensity factors.

Polynomial influence coefficients were determined for the same numerical models. These were used to validate the $K_{I}$ values determined for an internally pressurized pipe bend, to where they were shown to agree to within $2.5 \%$ difference. The use of the polynomial influence coefficients has further been demonstrated with the determination of $K_{I}$ values resulting from the residual stresses in a pressurized cracked curved tubing which has been subjected to varying degrees of overstrain. 


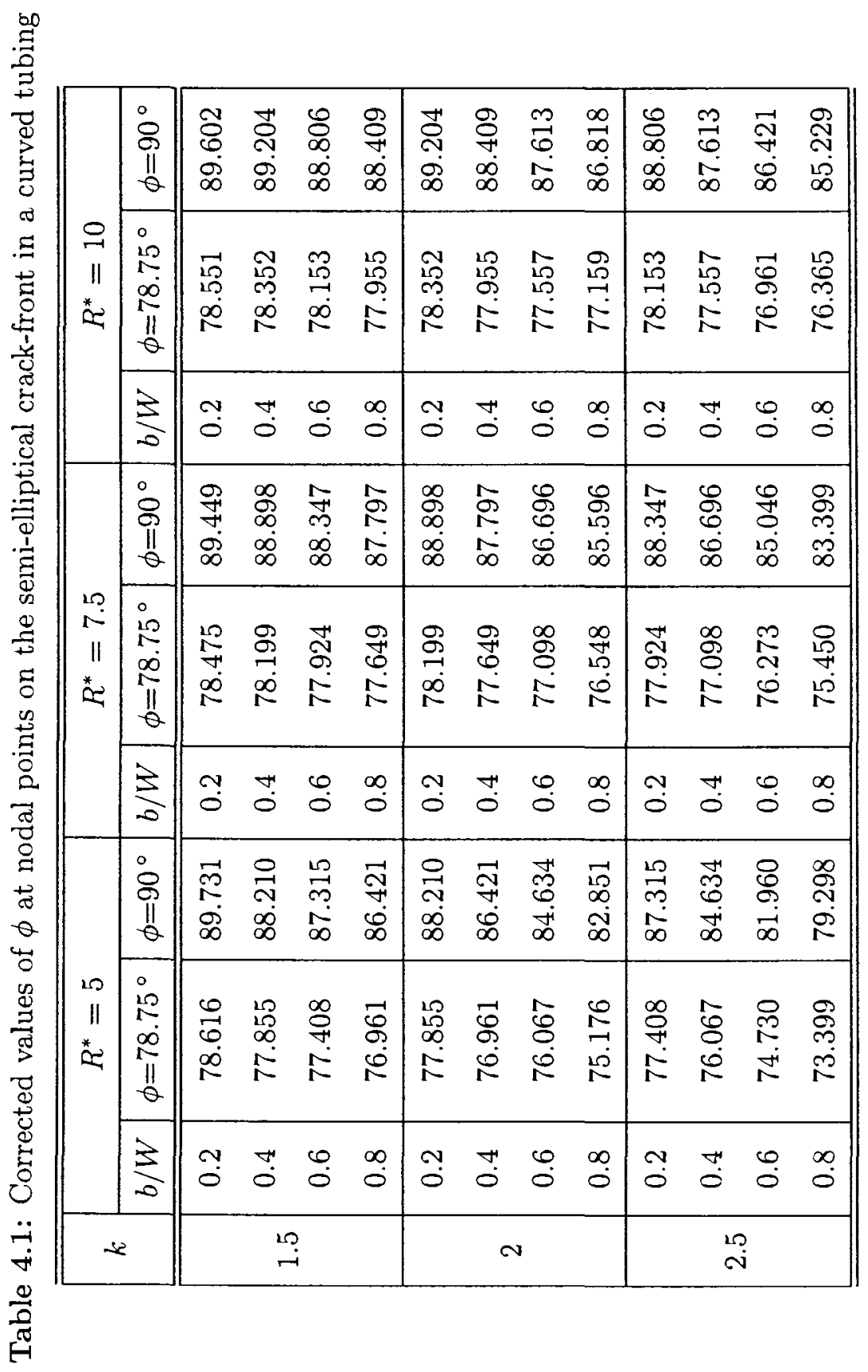




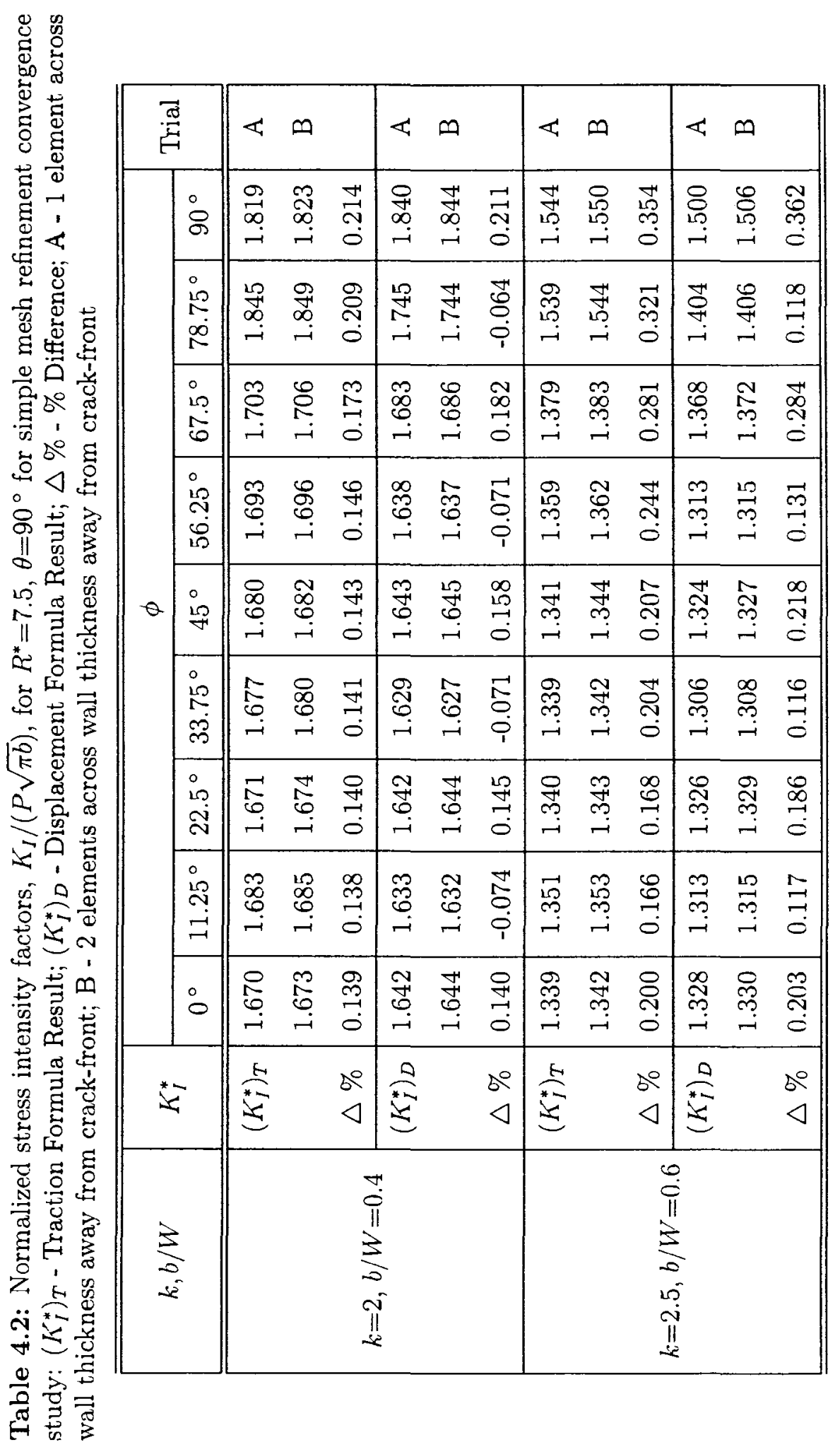




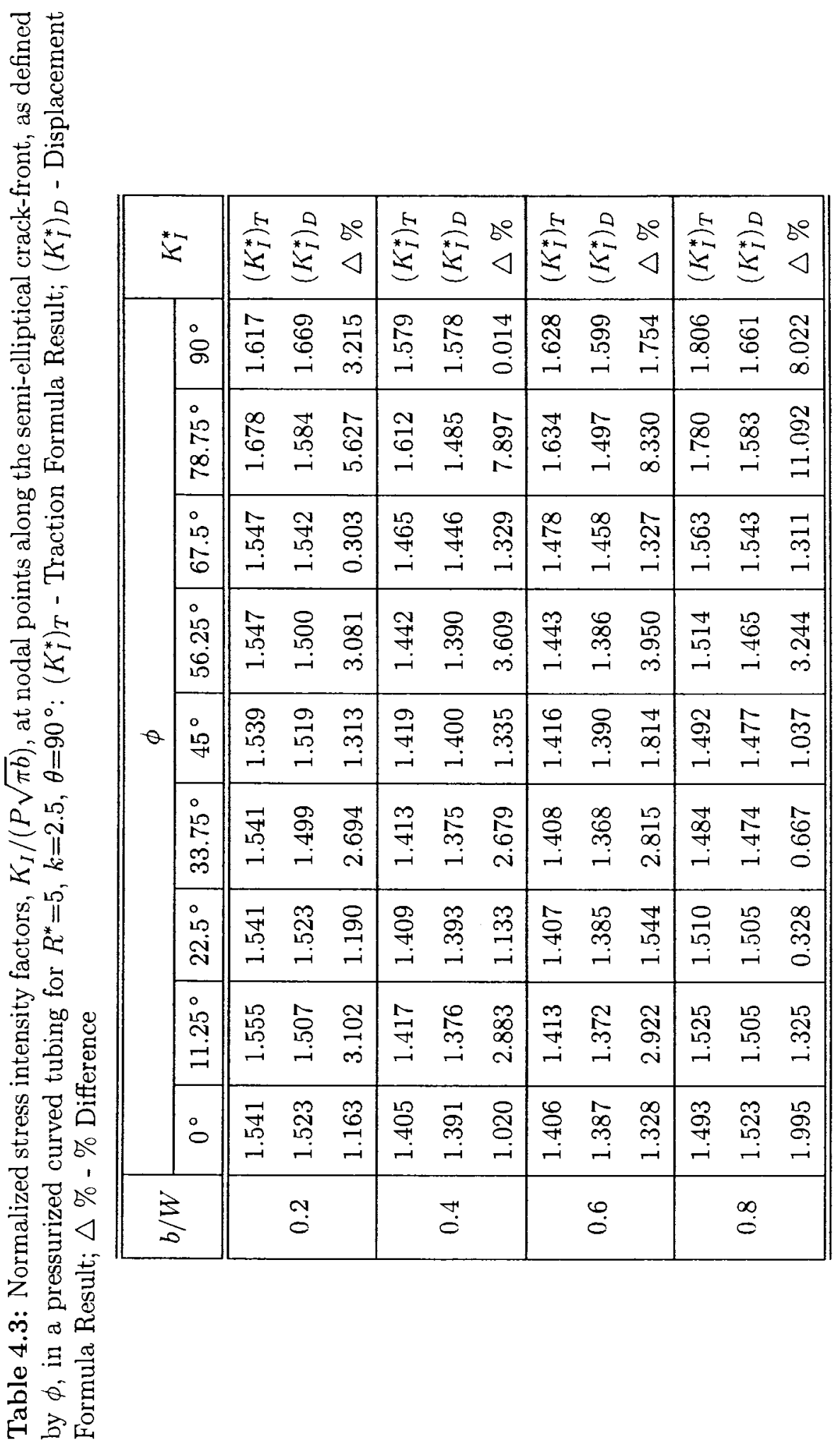




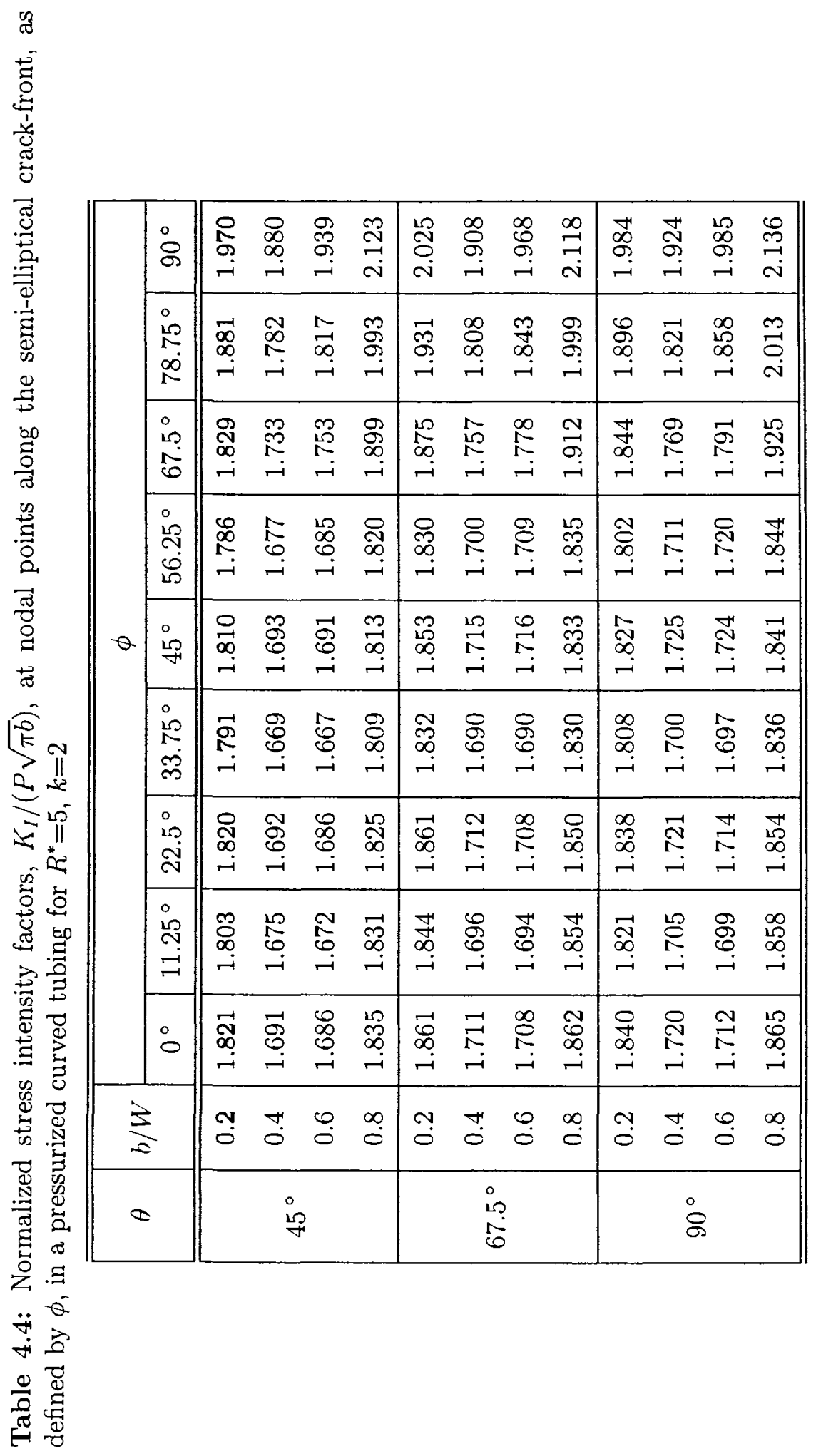


Table 4.5: Polynomial coefficients to define hoop stress distribution in internally pressurized uncracked curved tubing with $R^{*}=5$

\begin{tabular}{|c|c|c|c|c|c|}
\hline \hline$k$ & $\theta$ & $A_{0}$ & $A_{1}$ & $A_{2}$ & $A_{3}$ \\
\hline \hline \multirow{3}{*}{1.5} & $45^{\circ}$ & 3.1149 & -1.7202 & 0.5849 & -0.0465 \\
& $67.5^{\circ}$ & 3.1768 & -1.8703 & 0.6352 & -0.0561 \\
& $90^{\circ}$ & 3.2087 & -1.9469 & 0.6548 & -0.0591 \\
\hline \multirow{3}{*}{2} & $45^{\circ}$ & 1.8888 & -1.9335 & 1.1159 & -0.1931 \\
& $67.5^{\circ}$ & 1.9418 & -2.0262 & 1.1720 & -0.2129 \\
& $90^{\circ}$ & 1.9861 & -2.1006 & 1.1969 & -0.2145 \\
\hline \multirow{3}{*}{2.5} & $45^{\circ}$ & 1.5376 & -2.6605 & 2.6521 & -0.9936 \\
& $67.5^{\circ}$ & 1.5853 & -2.7645 & 2.8027 & -1.0771 \\
& $90^{\circ}$ & 1.6302 & -2.8602 & 2.9079 & -1.1292 \\
\hline \hline
\end{tabular}

Table 4.6: Polynomial coefficients to define hoop stress distribution in internally pressurized uncracked curved tubing with $R^{*}=7.5$

\begin{tabular}{|c|c|c|c|c|c|}
\hline \hline$k$ & $\theta$ & $A_{0}$ & $A_{1}$ & $A_{2}$ & $A_{3}$ \\
\hline \hline \multirow{4}{*}{1.5} & $\mathbf{4 5}$ & 2.9040 & -1.6419 & 0.5987 & -0.0649 \\
& $\mathbf{6 7 . 5 ^ { \circ }}$ & 2.9073 & -1.8602 & 0.9906 & -0.2642 \\
& $\mathbf{9 0}^{\circ}$ & 2.9229 & -1.8832 & 0.9926 & -0.2643 \\
\hline \multirow{3}{*}{2} & $\mathbf{4 5 ^ { \circ }}$ & 1.8498 & -2.2673 & 1.8484 & -0.6209 \\
& $67.5^{\circ}$ & 1.8350 & -2.4042 & 2.1609 & -0.7997 \\
& $90^{\circ}$ & 1.8883 & -2.3433 & 1.8929 & -0.6366 \\
\hline \multirow{3}{*}{2.5} & $\mathbf{4 5}$ & 1.4821 & -2.6276 & 2.6308 & -0.9962 \\
& $\mathbf{6 7 . 5 ^ { \circ }}$ & 1.5133 & -2.6881 & 2.6863 & -1.0211 \\
& $90^{\circ}$ & 1.5198 & -2.7120 & 2.7158 & -1.0359 \\
\hline \hline
\end{tabular}


Table 4.7: Polynomial coefficients to define hoop stress distribution in internally pressurized uncracked curved tubing with $R^{*}=10$

\begin{tabular}{|c|c|c|c|c|c|}
\hline \hline$k$ & $\theta$ & $A_{0}$ & $A_{1}$ & $A_{2}$ & $A_{3}$ \\
\hline \hline \multirow{4}{*}{1.5} & $45^{\circ}$ & 2.7984 & -1.7521 & 0.9365 & -0.2440 \\
& $67.5^{\circ}$ & 2.8111 & -1.7724 & 0.9393 & -0.2445 \\
& $90^{\circ}$ & 2.8128 & -1.7853 & 0.9509 & -0.2491 \\
\hline \multirow{3}{*}{2} & $45^{\circ}$ & 1.8189 & -2.2506 & 1.8034 & -0.5963 \\
& $67.5^{\circ}$ & 1.8066 & -2.2595 & 1.8380 & -0.6168 \\
& $90^{\circ}$ & 1.7875 & -2.2603 & 1.8728 & -0.6375 \\
\hline \multirow{3}{*}{2.5} & $45^{\circ}$ & 1.4488 & -2.6134 & 2.6204 & -0.9943 \\
& $67.5^{\circ}$ & 1.4550 & -2.6281 & 2.6325 & -0.9999 \\
& $90^{\circ}$ & 1.4566 & -2.6373 & 2.6449 & -1.0061 \\
\hline \hline
\end{tabular}




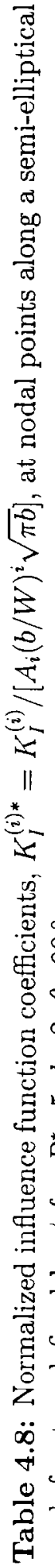

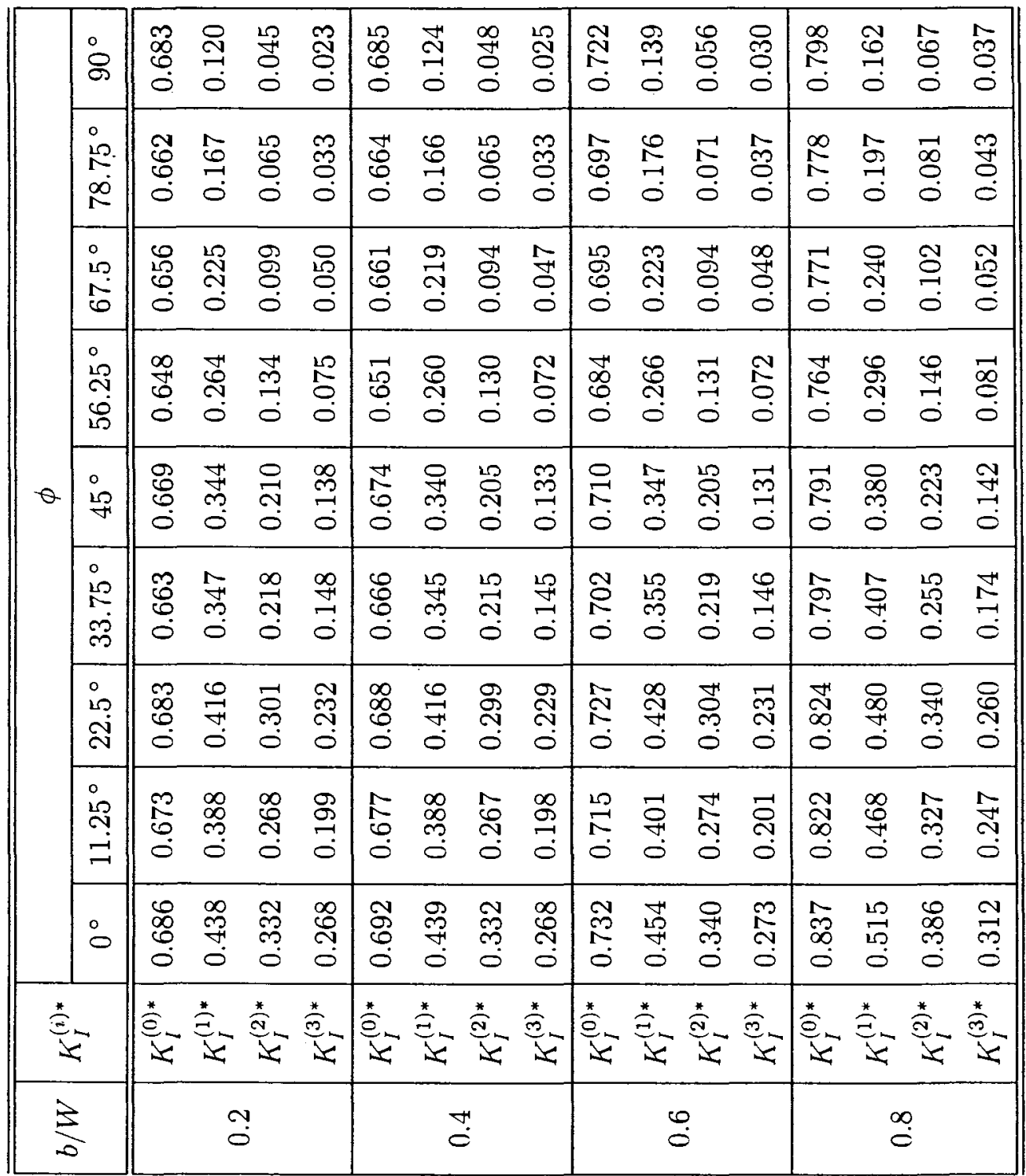

先

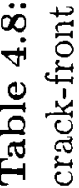




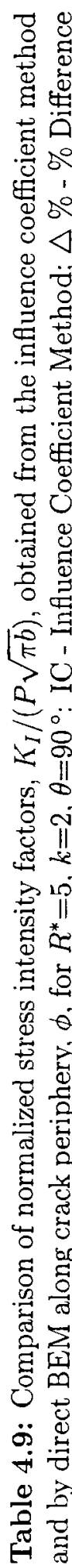

\begin{tabular}{|c|c|c|c|c|c|c|c|c|c|c|c|c|c|}
\hline \multicolumn{2}{|c|}{ 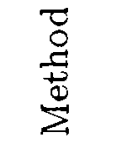 } & 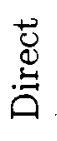 & $\stackrel{\varrho}{\varrho}$ & $\begin{array}{l}\Delta 0^{\circ} \\
\Delta\end{array}$ & 总 & $\varrho$ & $\stackrel{\Delta}{\Delta}$ & 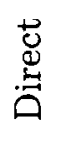 & $\varrho$ & $\begin{array}{l}\Delta 0 \\
4\end{array}$ & 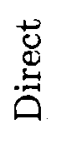 & $\stackrel{\circlearrowright}{\varrho}$ & $\begin{array}{l}\Delta^{\circ} \\
\triangleleft\end{array}$ \\
\hline \multirow{9}{*}{$\theta$} & $\stackrel{8}{8}$ & $\stackrel{\vec{D}}{\stackrel{\infty}{\sigma}}$ & $\underset{\mathscr{\delta}}{\stackrel{-}{-}}$ & \begin{tabular}{l}
$\stackrel{8}{\%}$ \\
\hdashline \\
0
\end{tabular} & 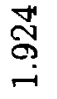 & 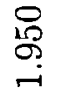 & $\underset{\sim}{\stackrel{N}{N}}$ & $\begin{array}{l}18 \\
0 \\
0 \\
0 \\
-10\end{array}$ & 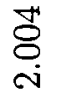 & $\begin{array}{l}\infty \\
\stackrel{1}{0} \\
\stackrel{9}{0} \\
0\end{array}$ & $\begin{array}{l}\stackrel{0}{-} \\
\stackrel{\sim}{N}\end{array}$ & 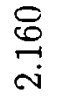 & $\stackrel{\mathscr{P}}{\vec{\rightleftarrows}}$ \\
\hline & 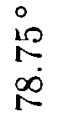 & 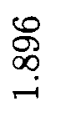 & $\overrightarrow{\vec{\sigma}}$ & $\begin{array}{l}\widetilde{N} \\
\infty \\
0 \\
0\end{array}$ & 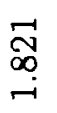 & 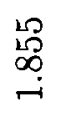 & 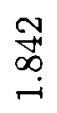 & $\begin{array}{l}\infty \\
1 \\
1 \\
\infty \\
-1 \\
-i\end{array}$ & 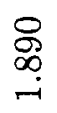 & $\stackrel{\infty}{\Gamma}$ & $\begin{array}{l}\stackrel{M}{O} \\
\stackrel{\sim}{\sim}\end{array}$ & 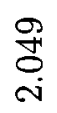 & $\underset{r}{\stackrel{H}{2}}$ \\
\hline & جن & 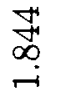 & 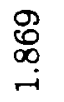 & గ్లి & 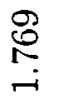 & $\stackrel{\infty}{\infty}$ & $\underset{\sim}{\stackrel{\infty}{\sim}}$ & న & $\begin{array}{l}\mathscr{M} \\
\stackrel{\infty}{\infty} \\
\stackrel{-}{-}\end{array}$ & $\underset{\sim}{\stackrel{\sigma}{\sim}}$ & 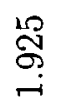 & $\stackrel{?}{?}$ & $\begin{array}{l}\overrightarrow{0} \\
\text { ల్ } \\
\dot{\sim}\end{array}$ \\
\hline & $\begin{array}{l}0 \\
\text { 오 } \\
6 \\
10\end{array}$ & $\begin{array}{l}\vec{\circ} \\
\stackrel{\infty}{-} \\
-i\end{array}$ & $\begin{array}{l}\stackrel{P}{\infty} \\
\infty \\
-i\end{array}$ & 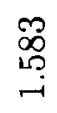 & $\vec{F}$ & $\underset{+}{\stackrel{g}{+}}$ & $\underset{\sim}{\stackrel{m}{\sim}}$ & $\underset{-i}{\stackrel{\text { న }}{-i}}$ & $\stackrel{\overrightarrow{0}}{\stackrel{0}{r}}$ & $\begin{array}{l}\text { ஓ్ల } \\
\text { ণj }\end{array}$ & $\begin{array}{l}\underset{\mathbb{H}}{\mathbb{S}} \\
\stackrel{-}{-}\end{array}$ & $\begin{array}{l}\hat{\infty} \\
\infty \\
\\
\end{array}$ & $\begin{array}{l}\infty \\
\stackrel{\infty}{\sim} \\
\sim\end{array}$ \\
\hline & is & 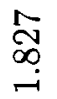 & $\begin{array}{l}\mathscr{P} \\
\mathscr{D} \\
\stackrel{-1}{-1}\end{array}$ & חై & $\stackrel{\mathscr{P}}{\stackrel{2}{N}}$ & $\stackrel{\vec{C}}{\stackrel{C}{-}}$ & 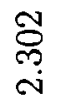 & $\underset{\mathbb{I}}{\stackrel{\Xi}{\sim}}$ & $\stackrel{8}{\mathscr{R}}$ & $\begin{array}{l}\vec{D} \\
\stackrel{\infty}{\infty} \\
\stackrel{N}{N}\end{array}$ & $\underset{\infty}{\vec{\infty}}$ & $\begin{array}{l}\mathscr{0} \\
\infty \\
\infty \\
-1\end{array}$ & 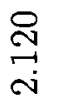 \\
\hline & 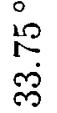 & $\begin{array}{l}\infty \\
\infty \\
\infty \\
-i \\
-i\end{array}$ & 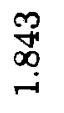 & $\begin{array}{l}\infty \\
\stackrel{8}{\circ} \\
\stackrel{-}{-1}\end{array}$ & 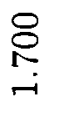 & $\begin{array}{l}\infty \\
\stackrel{\infty}{\sim} \\
\stackrel{-}{-}\end{array}$ & $\underset{\sim}{N}$ & 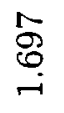 & $\stackrel{n}{n}$ & $\begin{array}{l}\text { O } \\
\text { लొ } \\
\text { oj }\end{array}$ & $\begin{array}{l}\infty \\
\infty \\
\infty \\
-i\end{array}$ & 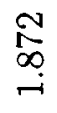 & ל్ֶ \\
\hline & $\begin{array}{l}\text { in } \\
\text { నิ }\end{array}$ & $\begin{array}{l}\infty \\
\underset{\infty}{\infty} \\
\stackrel{-}{\infty}\end{array}$ & $\underset{\substack{\infty \\
\infty}}{\stackrel{\infty}{\infty}}$ & $\frac{R}{i}$ & $\underset{\sim}{\vec{N}}$ & $\stackrel{8}{8}$ & 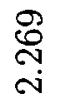 & $\underset{\sim}{\stackrel{\rightleftarrows}{\vec{r}}}$ & $\begin{array}{l}\overrightarrow{10} \\
\stackrel{2}{r}\end{array}$ & ס & 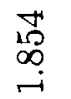 & $\begin{array}{l}\infty \\
\infty \\
\infty \\
-1\end{array}$ & $\underset{\sim}{\stackrel{J}{*}}$ \\
\hline & 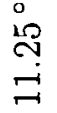 & $\begin{array}{l}\overrightarrow{\widetilde{O}} \\
\stackrel{-}{-1}\end{array}$ & 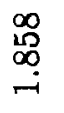 & 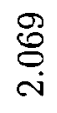 & 崖 & $\underset{r}{\stackrel{\vec{r}}{r}}$ & $\begin{array}{l}\stackrel{N}{0} \\
\text { ণ }\end{array}$ & 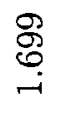 & 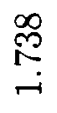 & 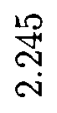 & $\begin{array}{l}\infty \\
1 \\
\infty \\
\infty \\
-i\end{array}$ & 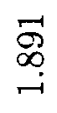 & $\begin{array}{l}\text { S) } \\
\stackrel{10}{1} \\
\stackrel{-1}{-1}\end{array}$ \\
\hline & ○ & 옹 & $\begin{array}{l}8 \\
\infty \\
\infty \\
-i\end{array}$ & 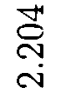 & 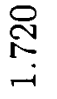 & 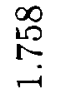 & $\begin{array}{l}\stackrel{\mathscr{N}}{N} \\
\text { }\end{array}$ & $\underset{ت}{\stackrel{\overbrace{}}{\sim}}$ & స્ & $\begin{array}{l}\stackrel{H}{0} \\
\stackrel{N}{S}\end{array}$ & \begin{tabular}{l}
18 \\
$\varnothing$ \\
$\infty$ \\
\hdashline
\end{tabular} & $\begin{array}{l}\mathscr{\$} \\
\stackrel{\infty}{\infty} \\
-1\end{array}$ & تُ \\
\hline \multicolumn{2}{|c|}{$\stackrel{3}{3}$} & & $\stackrel{\sim}{\circ}$ & & & $\stackrel{\ddot{0}}{\circ}$ & & & $\stackrel{0}{0}$ & & & $\begin{array}{l}\infty \\
0 \\
0\end{array}$ & \\
\hline
\end{tabular}




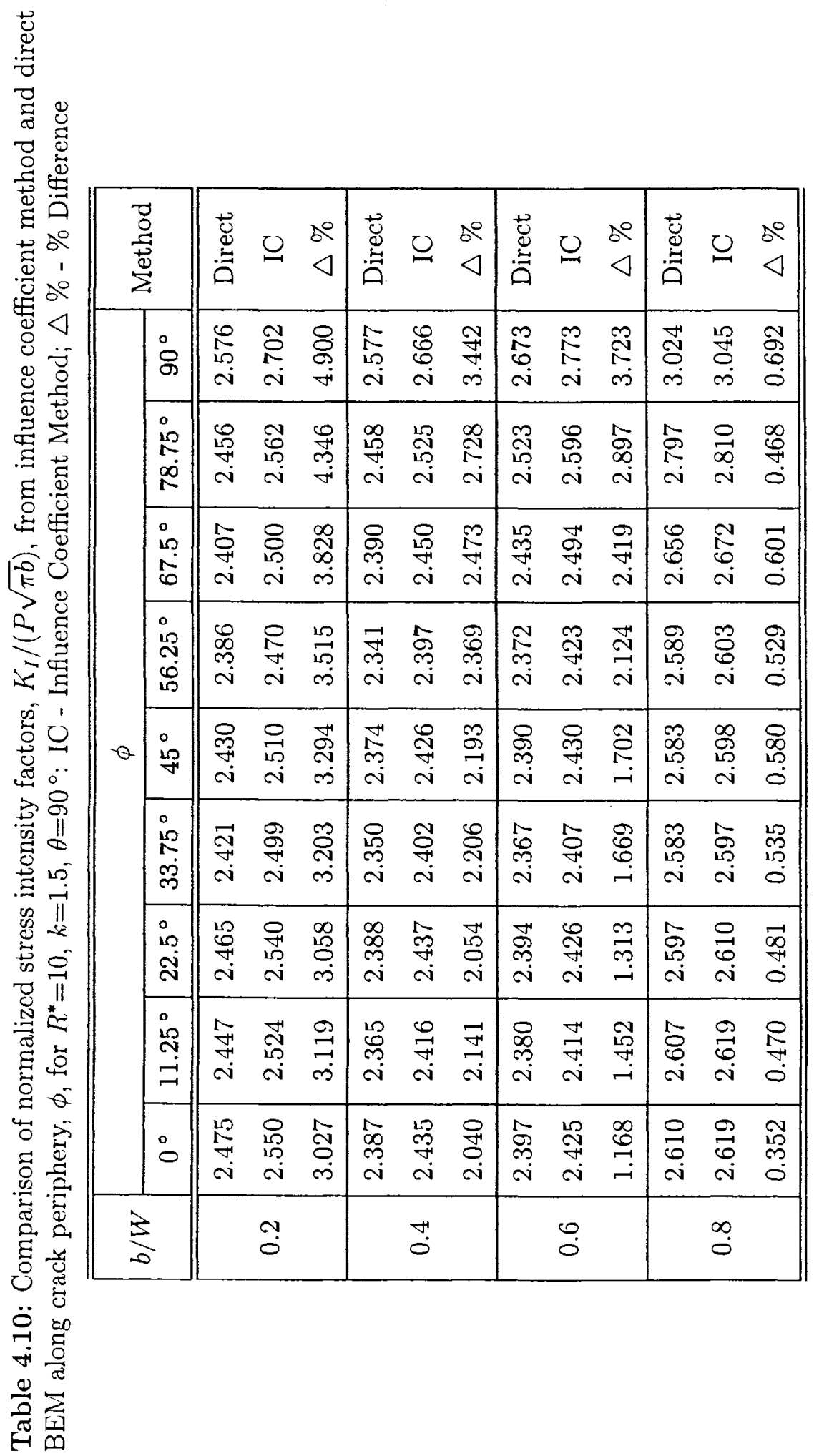




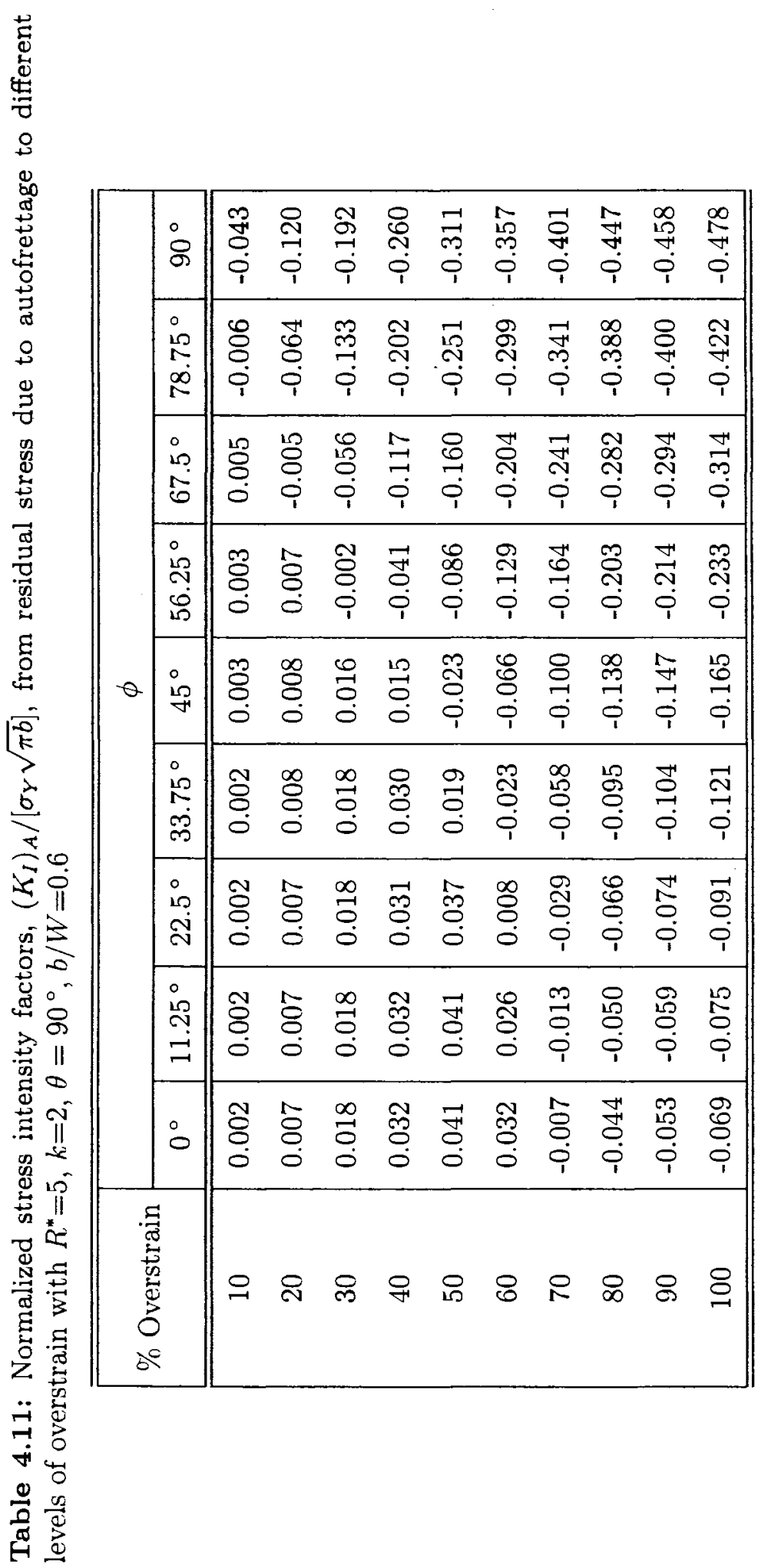




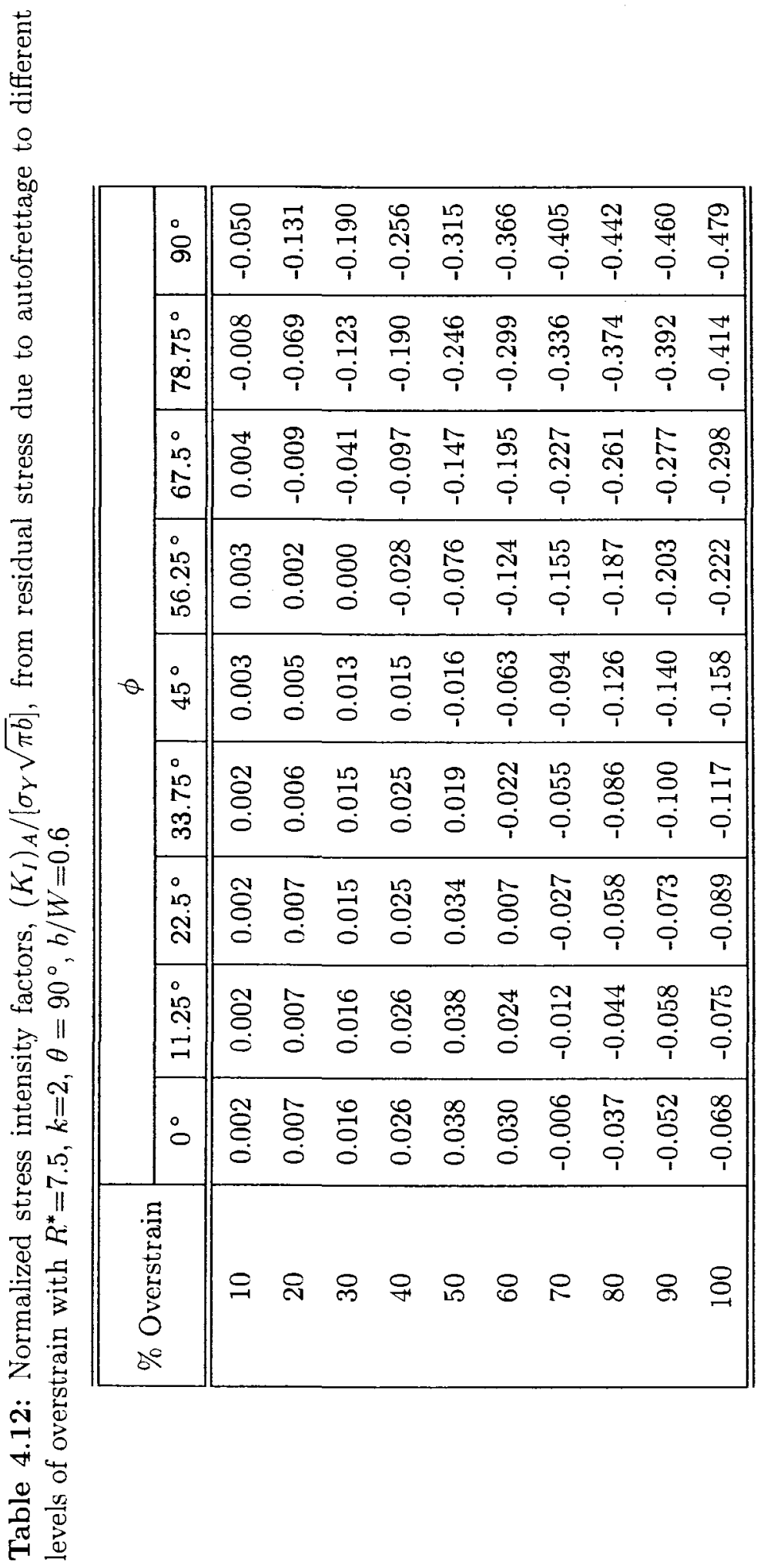




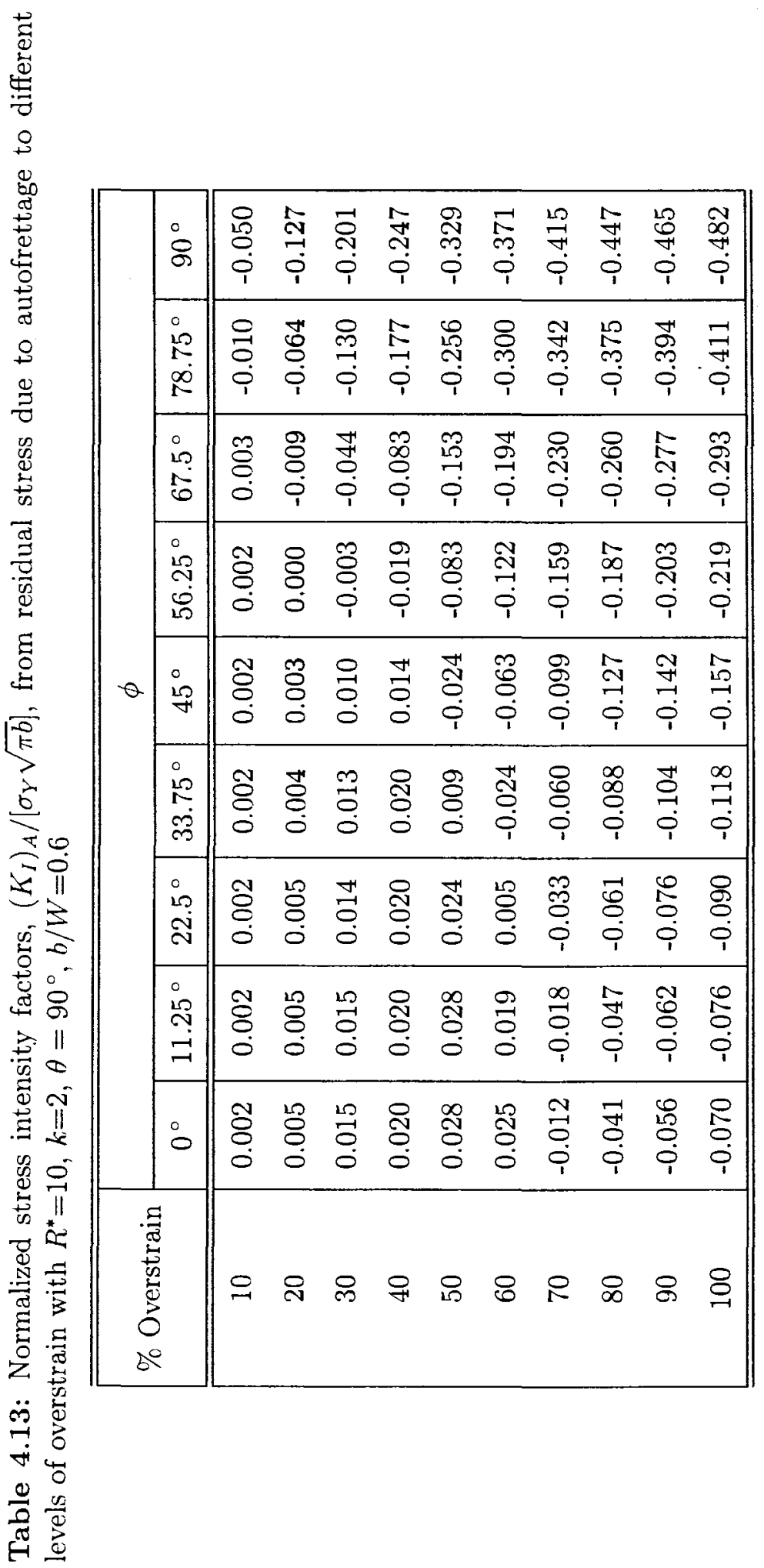




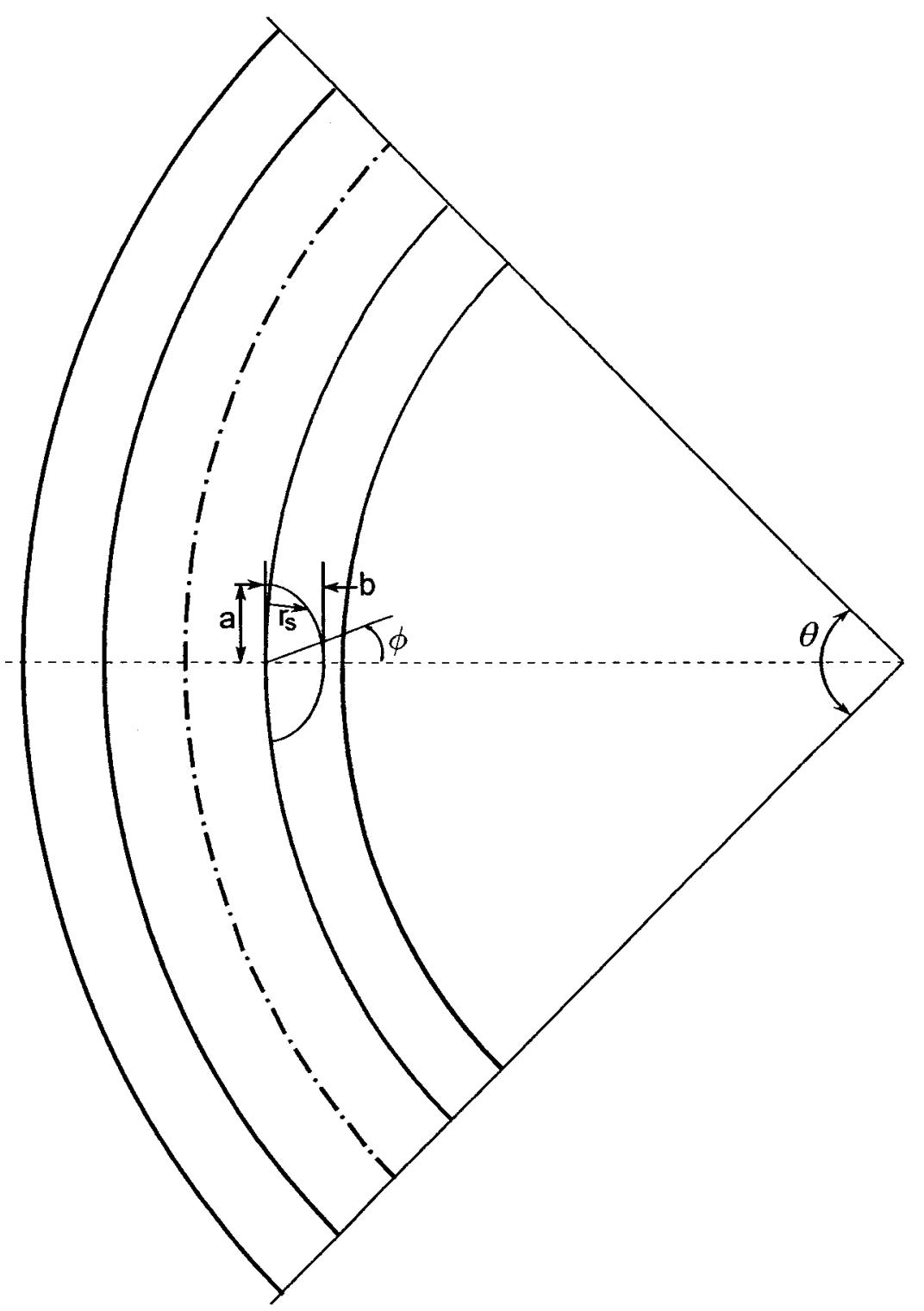

Figure 4.1: Semi-elliptical crack in curved tubing 


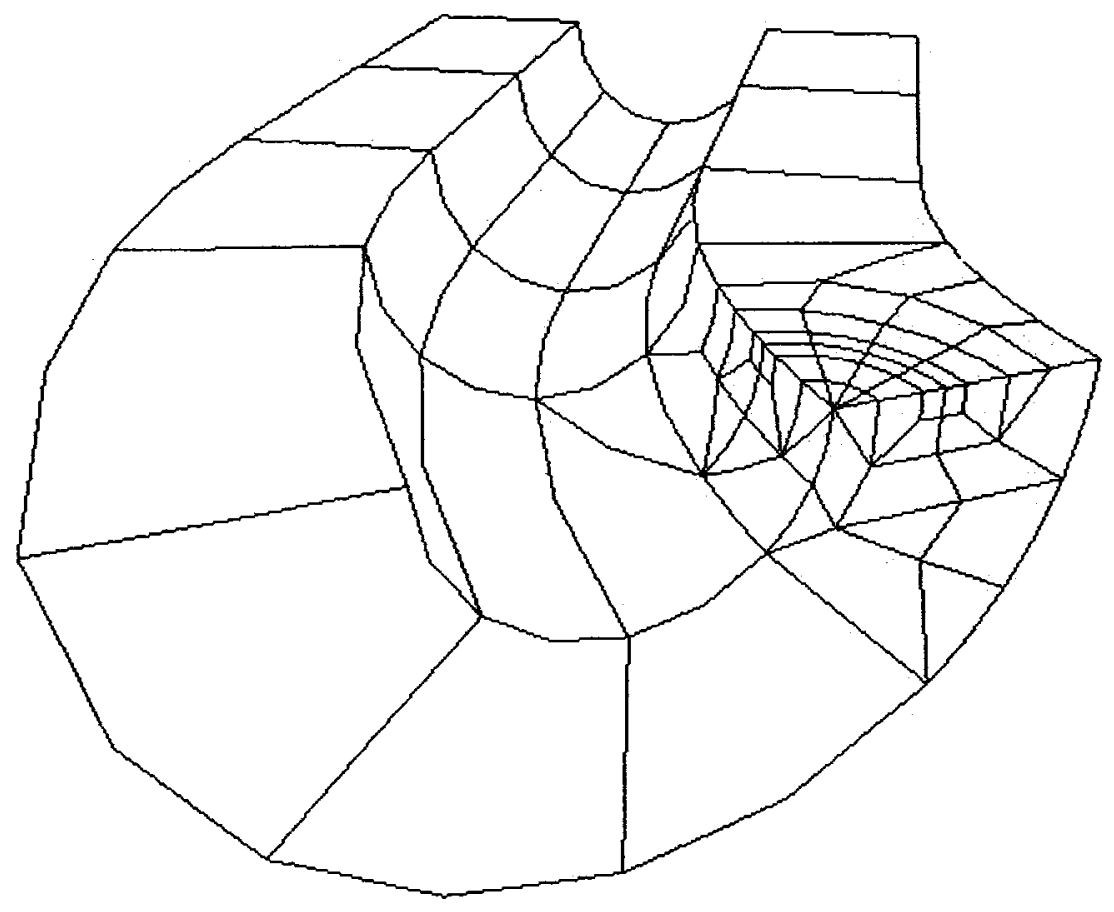

Figure 4.2: Sample BEM mesh for $R^{*}=7.5, k=2.5, \theta=67.5^{\circ}, b / W=0.4$ with 112 elements and 332 nodes 


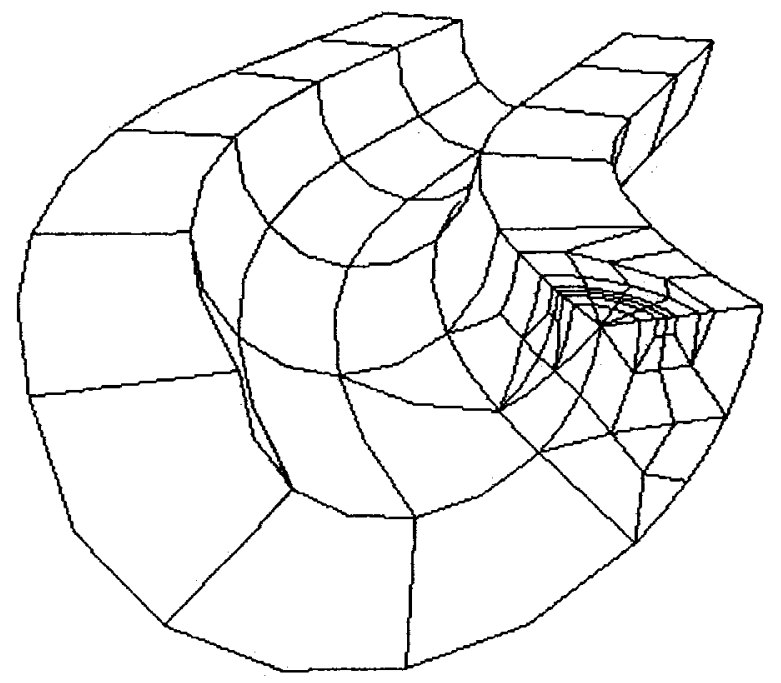

(a) Trial A

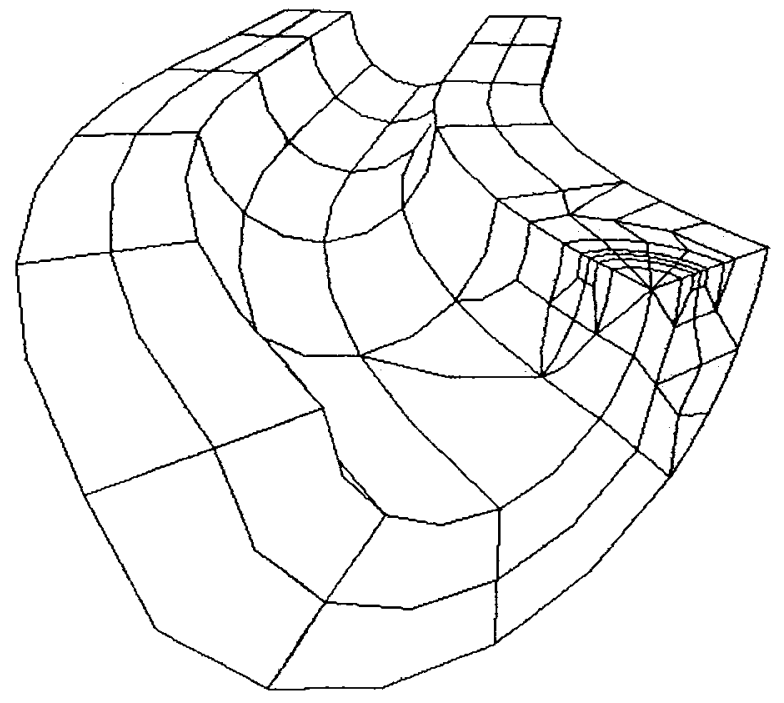

(b) Trial $\mathrm{B}$

Figure 4.3: BEM discretization for mesh refinement study 


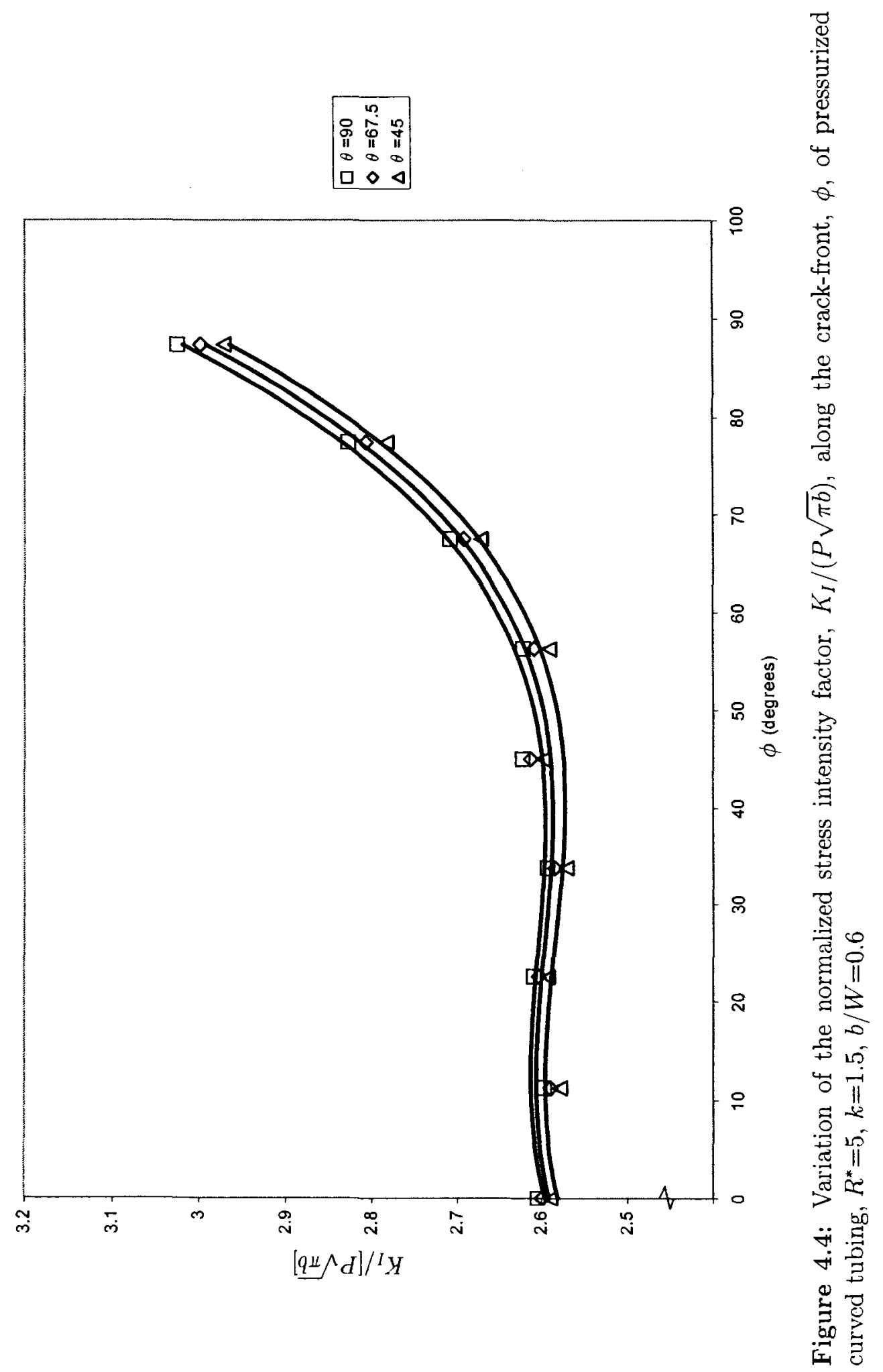




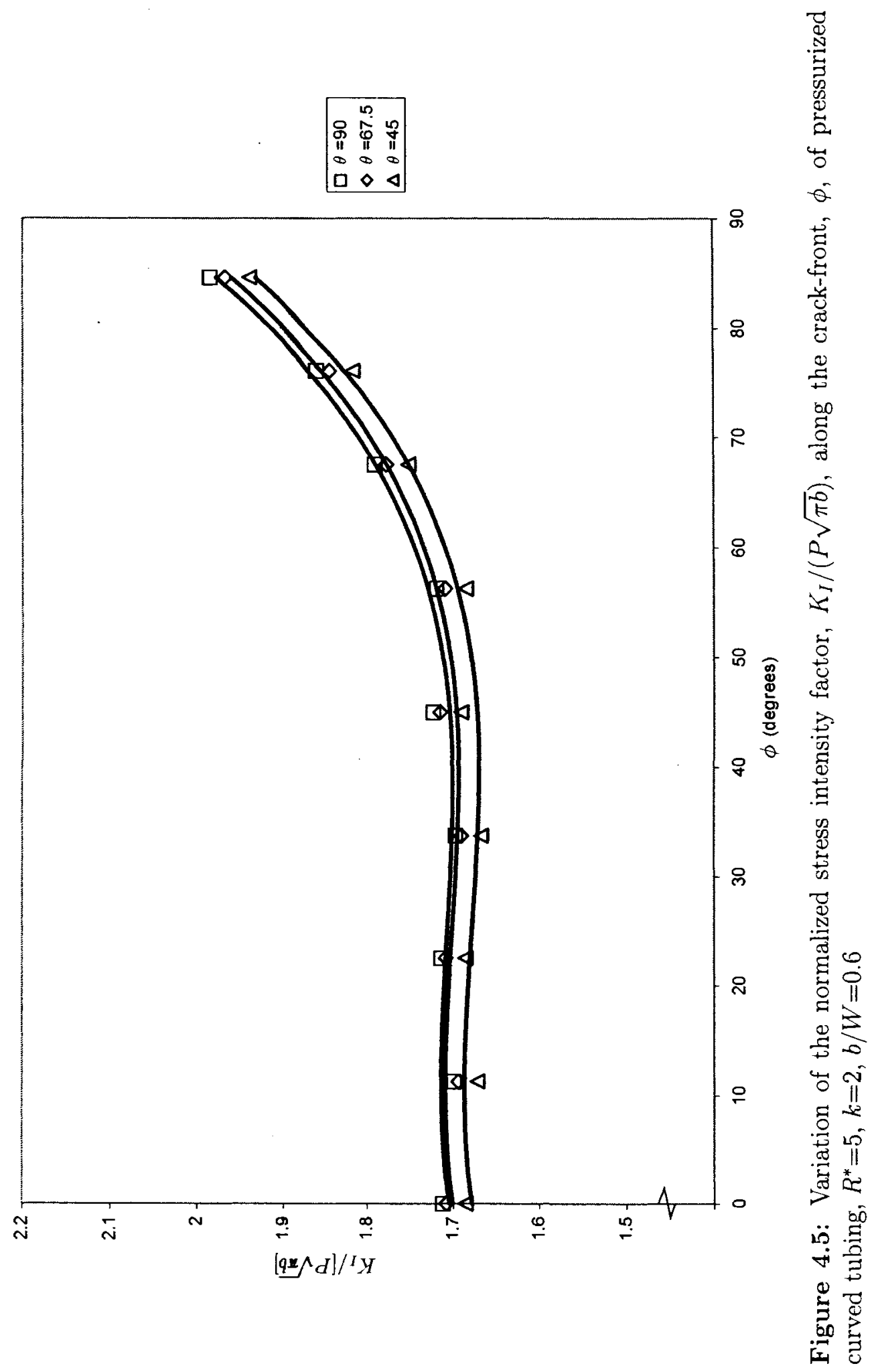




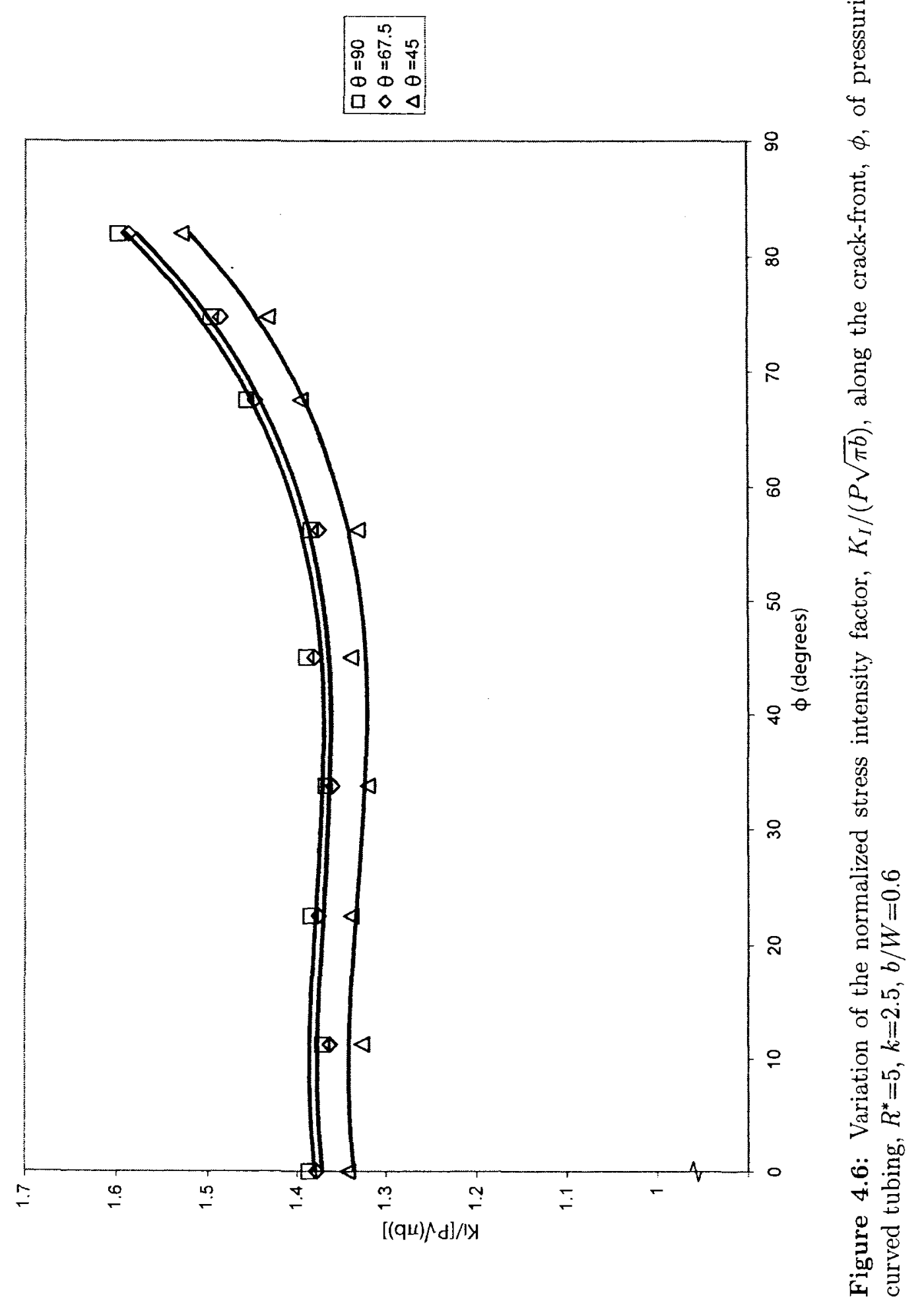




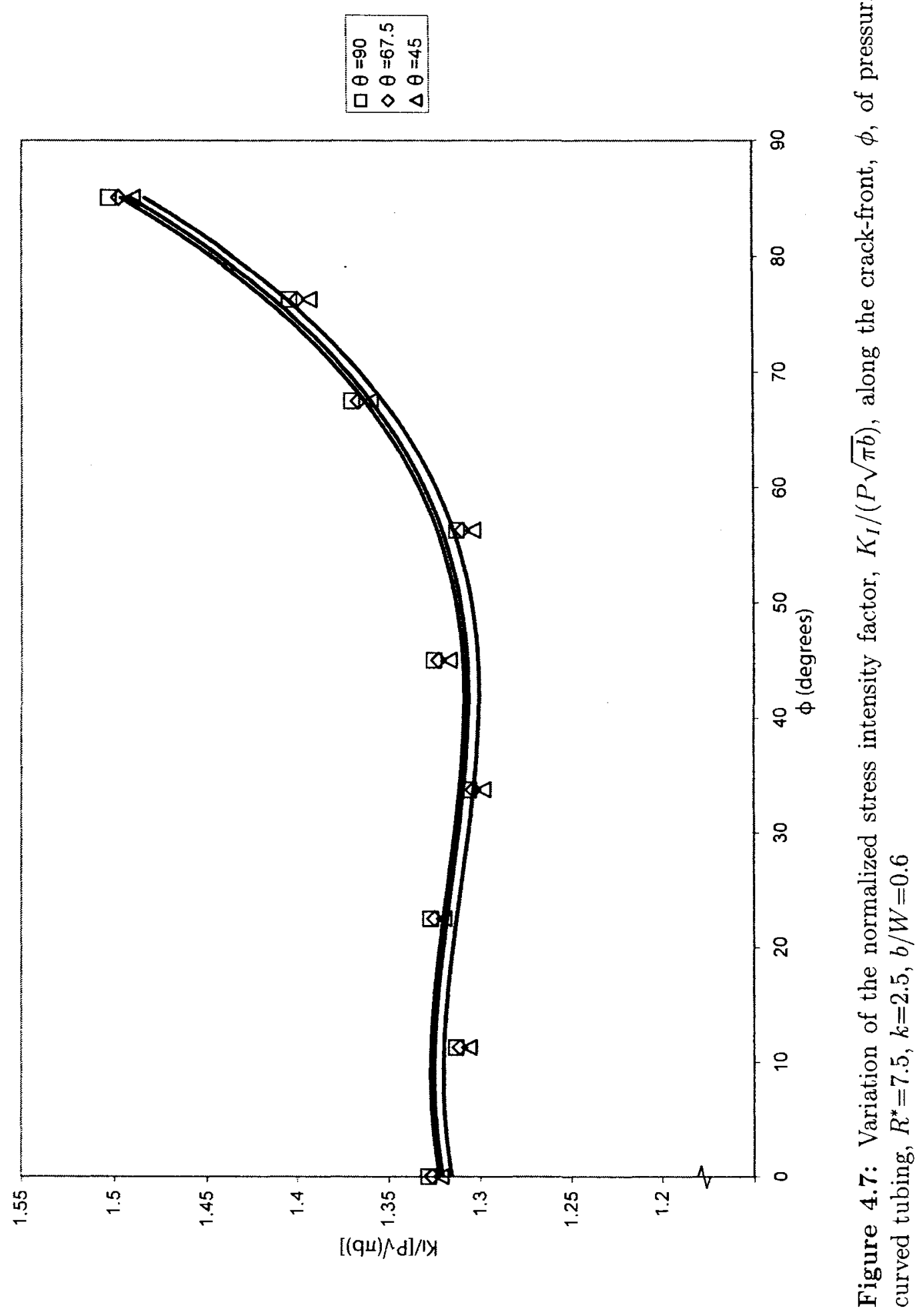




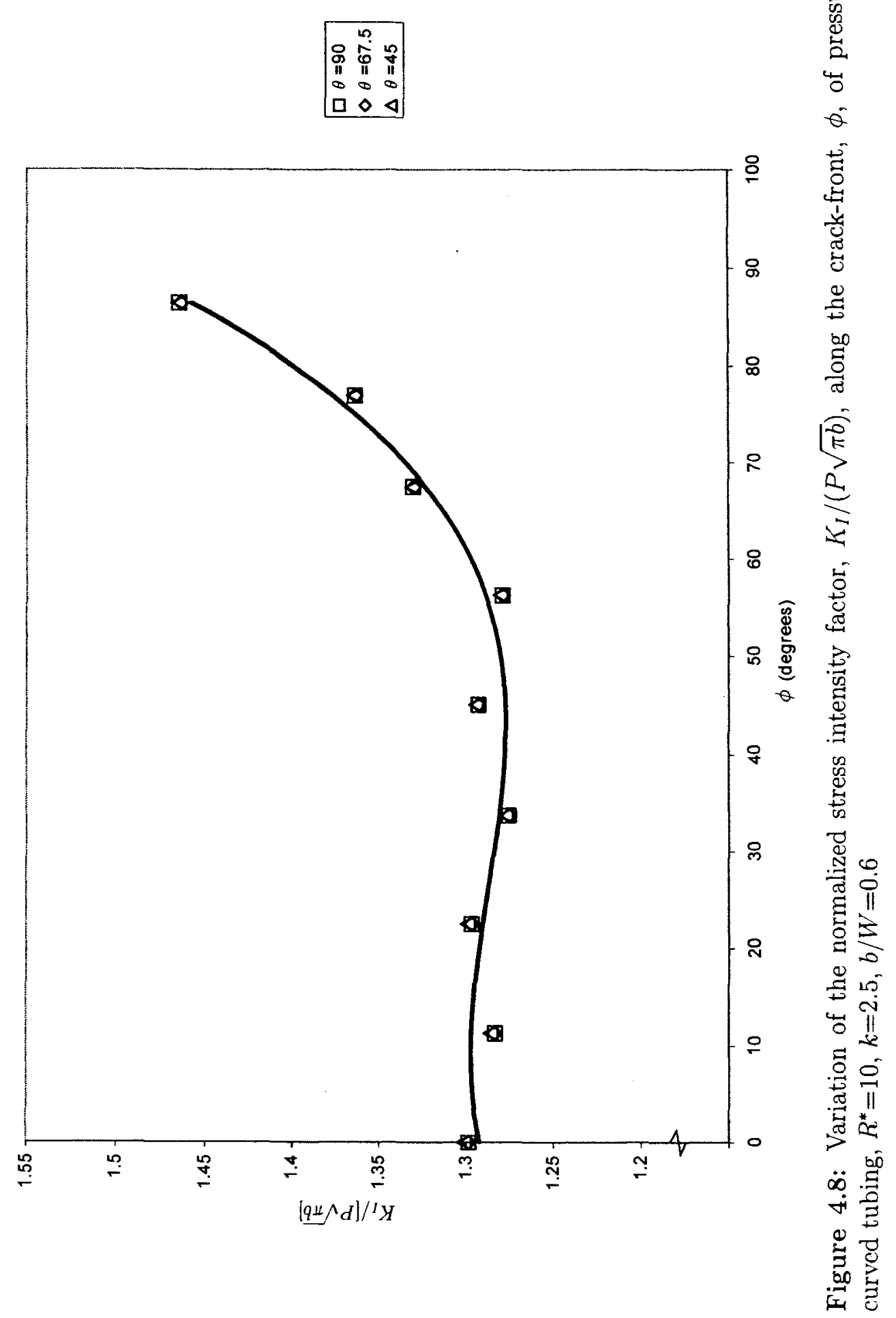




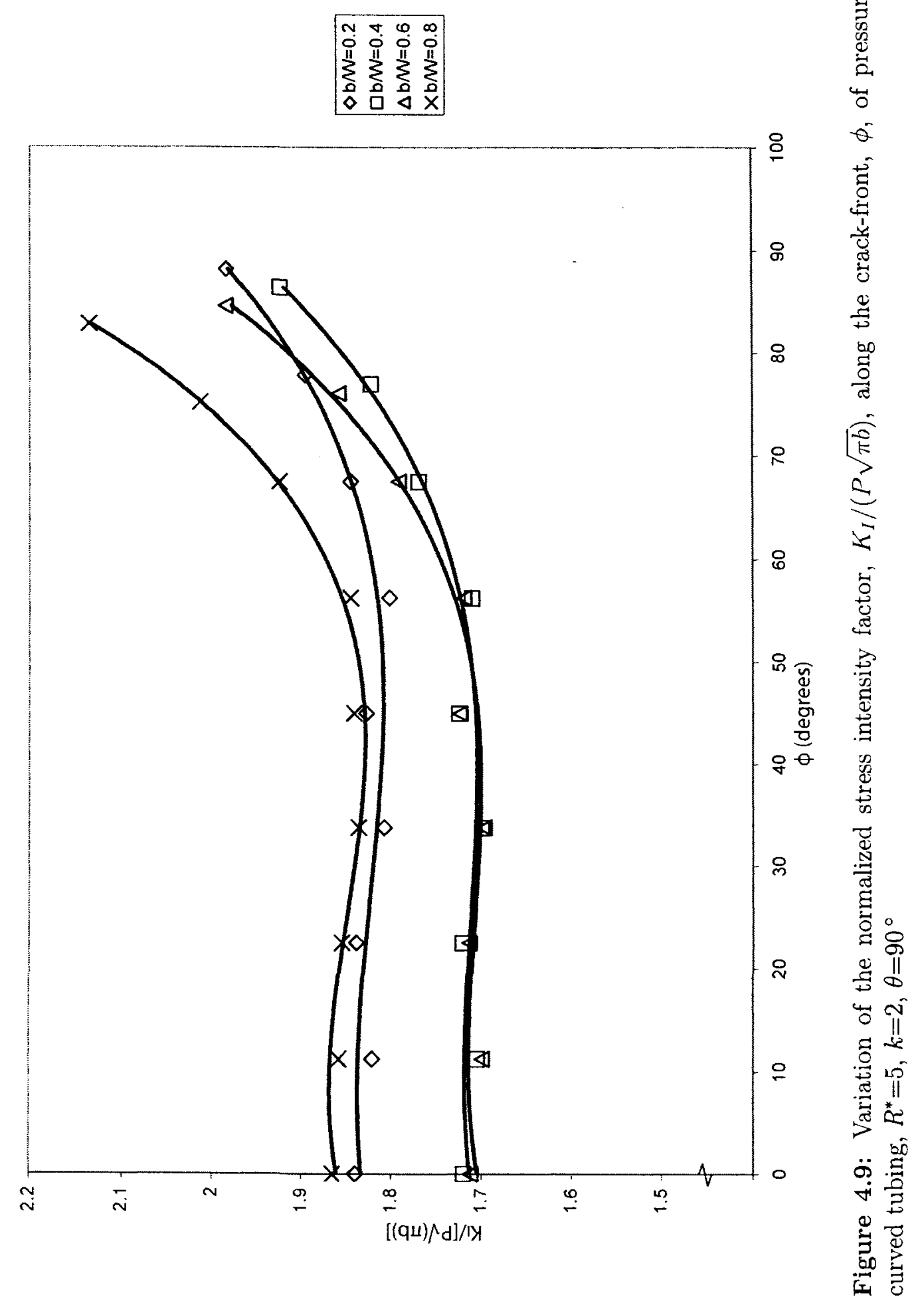




\section{Chapter 5}

\section{Conclusions}

Throughout this thesis, internally pressurized thick-walled curved tubings were investigated using the boundary element method (BEM). Before analyzing these components, the three-dimensional BEM was reviewed and its efficiency in solving stress concentration and linear elastic fracture mechanics problems was demonstrated with some examples.

In this study, a thick-walled curved tubing subjected to internal pressure has been investigated over the parameters of bend radius ratio $R^{*}=R / R_{1}$, radius ratio of the cross-section $k=R_{2} / R_{1}$, and angle of curvature $\theta$. They were: $R^{*}=5,7.5,10 ; k=1.5$, $2.0,2.5 ; \theta=45^{\circ}, 67.5^{\circ}, 90^{\circ}$. It was determined that the normalized hoop stress factor, $\sigma_{\psi} / \sigma_{o}$, was greatest at the intrados of the pipe bend, which is consistent with previously reported toroidal analyses in the literature. It was also observed that the normalized stress factor was greater for lower $R_{2} / R_{1}$ radius ratios, smaller bend radius ratio $R / R_{1}$, and larger angles of curvature. Additionally, the effect of decreasing the angle of curvature, $\theta$, was found to be minimal, particularly for larger bend radii.

A semi-elliptical crack of $b / a=0.8$ has been modelled at the intrados and investigated over four relative crack depths, namely, $b / W=0.2,0.4,0.6$, and 0.8 . Normalized stress intensity factors have been obtained along the crack periphery, $\phi$. It was observed that the normalized stress intensity factor remained fairly constant up to about 
$\phi=60^{\circ}$, at which point it increased rapidly towards the free surface. The normalized stress intensity factors obtained were also observed to be greater for relative crack depths 0.2 and 0.8 than for 0.4 and 0.6 ; they were observed to increase more rapidly with proximity to the free surface for increasing crack depth.

To ascertain the veracity of the stress intensity factor results obtained from the direct BEM, polynomial influence coefficients have been determined for each combination of geometric parameters and applied to the internally pressurized load case. These influence coefficients may be applied to solve for the stress intensity factor of any load case with the same cracked geometry. To demonstrate the versatility of the influence coefficient results, stress intensity factors due to the residual stresses arising from autofrettage of the curved tube have also been obtained. In this analysis, different levels of overstain, from 10 to $100 \%$ at $10 \%$ intervals, were investigated for a representative set of physical models.

The effects of non-uniformity of the cross-section, such as local thinning of the wall, ovalization, and local defects which may occur during manufacture have not been considered in this study. Clearly, they will have implications on the site of crack initiation and propagation. These can be a focus of further study. 


\section{References}

Aliabadi, M. H. \& Rooke, D. P. (1991), Numerical Fracture Mechanics, Kluwer Academic Publishers, Dordrecht.

Atluri, S. N. \& Kathiresan, K. (1979), '3d analyses of surface flaws in thick-walled reactor pressure-vessels using displacement-hybrid finite element method', Nuclear Engineering and Design 51, 163-176.

Barthélemy, J. (1947), 'Mémoire no. 867', Bull. Ass. Tech. Marit. 46, 411.

Becker, A. A. (1992), The Boundary Element Method in Engineering, McGraw-Hill.

Blackburn, W. S. \& Hellen, T. K. (1977), 'Calculation of stress intensity factors in three dimensions by finite element method', International Journal for Numerical Methods in Engineering 11, 211-229.

Colle, A., Redekop, D. \& Tan, C. L. (1987), 'Pressure loading and bending of hollow tori', Int. J. Pres. Ves. Piping 27, 137-154.

Cruse, T. A. (1969), 'Numerical solutions in three dimensional elastostatics', Int. J. Solids Structures 5, 1259-1274.

Hill, R. (1950), The Mathematical Theory of Plasticity, Oxford University Press.

Lang, H. A. (1984), 'Toroidal elastic stress fields for pressurized elbows and pipe bends', Int. J. Pres. Ves. Piping 15, 291-305.

Luchi, M. L. \& Poggialini, A. (1983), Computation of 3-dimensional stress intensity factors using special boundary elements, in C. Brebbia, ed., 'Proc. Fifth Int. Conf. on BEM', Spring-Verlag, Berlin, pp. 461-470.

Nadiri, F., Tan, C. L. \& Fenner, R. T. (1982), 'Three-dimensional analyses of surface cracks in pressurised thick-walled cylinders', Int. J. Pres. Ves. Piping 10, 159 167. 
Pu, S. L. \& Hussain, M. A. (1981), 'Residual stress redistribution caused by notches and cracks in a partially autofrettaged tube', Journal of Pressure Vessel Technology ASME 103, 302-306.

Reddy, J. N. (2006), An Introduction to the Finite Element Method, 3rd edn, McGrawHill, N.Y.

Rees, D. W. A. (2004), 'Autofrettage of thick-walled pipe bends', Int. J. of Mech. Sciences 46, 1675-1696.

Shim, M. L. (1986), Boundary integral equation stress analysis of cracked thick-walled cylinders, Master's thesis, Carleton University.

Sternberg, E. \& Sadowsky, M. A. (1949), 'Three-dimensional solution for the stress concentration around a circular hole in a plate of arbitrary thickness', Journal of Applied Mechanics 16, 27-38.

Swanson, S. A. V. \& Ford, H. (1959), 'Stresses in thick-walled plane pipe bends', $J$. Mech. Eng. Sci. 1(2), 103-112.

Tan, C. L. \& Fenner, R. T. (1978), 'Three-dimensional stress analysis by the boundary integral equation method', Journal of Strain Analysis 13(4), 213-219.

Tan, C. L. \& Fenner, R. T. (1979), Elastic fracture mechanics analysis by the boundary integral equation method, in 'Proc. R. Soc. Lond. A369', pp. 243-260.

Tan, C. L. \& Fenner, R. T. (1980), 'Stress intensity factors for semi-elliptical surface cracks in pressurized cylinders using the boundary integral equation method', International Journal of Fracture 16(3), 233-245.

Tan, C. L. \& Shim, M. L. (1986), 'Stress intensity factor influence coefficients for internal surface cracks in thick-walled cylinders', Int. J. Pres. Ves. and Piping 24, 49-72.

Thuloup, A. (1937), 'Mémoire no. 725', Bull. Ass. Tech. Marit. 41, 317.

Turner, C. E. \& Ford, H. (1957), Examination of the theories for calculating the stresses in pipe bends subjected to in-plane bending, in '1957 Proc. Instn Mech. Engrs, Lond.', p. 513.

Unger, D. J. (1995), Analytical Fracture Mechanics, Academic Press.

von Kármán, T. (1911), Z. Ver. dtsch. Ing. 55, 1889.

Zienkiewicz, O. C. (1977), The Finite Element Method, 3rd edn, McGraw-Hill, N.Y. 


\section{Appendix A}

\section{Shape Functions}

\section{A.1 Shape Functions for Triangular Elements}

Shape functions for a quadratic triangular element as shown in Figure 2.2 are given below.

$$
\begin{aligned}
& N^{1}\left(\xi_{1}, \xi_{2}\right)=\left(1-\xi_{1}-\xi_{2}\right)\left(1-2 \xi_{1}-2 \xi_{2}\right) \\
& N^{2}\left(\xi_{1}, \xi_{2}\right)=4 \xi_{1}\left(1-\xi_{1}-\xi_{2}\right) \\
& N^{3}\left(\xi_{1}, \xi_{2}\right)=\xi_{1}\left(2 \xi_{1}-1\right) \\
& N^{4}\left(\xi_{1}, \xi_{2}\right)=4 \xi_{1} \xi_{2} \\
& N^{5}\left(\xi_{1}, \xi_{2}\right)=\xi_{2}\left(2 \xi_{2}-1\right) \\
& N^{6}\left(\xi_{1}, \xi_{2}\right)=4 \xi_{2}\left(1-\xi_{1}-\xi_{2}\right)
\end{aligned}
$$




\section{A.2 Shape Functions for Quadrilateral Elements}

Shape functions for a quadratic element as shown in Figure 2.3 are given below.

$$
\begin{aligned}
& N^{1}\left(\xi_{1}, \xi_{2}\right)=-\frac{1}{4}\left(1-\xi_{1}\right)\left(1-\xi_{2}\right)\left(1+\xi_{1}+\xi_{2}\right) \\
& N^{2}\left(\xi_{1}, \xi_{2}\right)=\frac{1}{2}\left(1-\xi_{1}^{2}\right)\left(1-\xi_{2}\right) \\
& N^{3}\left(\xi_{1}, \xi_{2}\right)=-\frac{1}{4}\left(1+\xi_{1}\right)\left(1-\xi_{2}\right)\left(1-\xi_{1}+\xi_{2}\right) \\
& N^{4}\left(\xi_{1}, \xi_{2}\right)=\frac{1}{2}\left(1-\xi_{1}\right)\left(1-\xi_{2}^{2}\right) \\
& N^{5}\left(\xi_{1}, \xi_{2}\right)=\frac{1}{2}\left(1+\xi_{1}\right)\left(1-\xi_{2}^{2}\right) \\
& N^{6}\left(\xi_{1}, \xi_{2}\right)=-\frac{1}{4}\left(1-\xi_{1}\right)\left(1+\xi_{2}\right)\left(1+\xi_{1}-\xi_{2}\right) \\
& N^{7}\left(\xi_{1}, \xi_{2}\right)=\frac{1}{2}\left(1-\xi_{1}^{2}\right)\left(1+\xi_{2}\right) \\
& N^{8}\left(\xi_{1}, \xi_{2}\right)=-\frac{1}{4}\left(1+\xi_{1}\right)\left(1+\xi_{2}\right)\left(1-\xi_{1}-\xi_{2}\right)
\end{aligned}
$$




\section{A.3 Shape Functions for Crack-Front Elements}

The displacement and traction shape functions for the quadrilateral element shown in Figure 2.3 are as follows for the nodes 1-2-3 along the crack-front

$$
\begin{aligned}
& N_{d}^{1}\left(\xi_{1}, \xi_{2}\right)=\frac{1}{2}\left(1-\xi_{1}\right)\left[-\xi_{1}-\left(1-\frac{\sqrt{2}}{2} \xi_{1}\right) \sqrt{1+\xi 2}+\frac{\sqrt{2}}{2}\left(1+\xi_{2}\right)\right] \\
& N_{d}^{2}\left(\xi_{1}, \xi_{2}\right)=\left(1-\xi_{1}^{2}\right)\left[1-\frac{\sqrt{2}}{2} \sqrt{1+\xi_{2}}\right] \\
& N_{d}^{3}\left(\xi_{1}, \xi_{2}\right)=\frac{1}{2}\left(1+\xi_{1}\right)\left[\xi_{1}-\left(1+\frac{\sqrt{2}}{2} \xi_{1}\right) \sqrt{1+\xi 2}+\frac{\sqrt{2}}{2}\left(1+\xi_{2}\right)\right] \\
& N_{d}^{4}\left(\xi_{1}, \xi_{2}\right)=\frac{1}{2}\left(1+\xi_{1}\right)\left[(2+\sqrt{2}) \sqrt{1+\xi_{2}}-(1+\sqrt{2})\left(1+\xi_{2}\right)\right] \\
& N_{d}^{5}\left(\xi_{1}, \xi_{2}\right)=\frac{1}{4}\left(1+\xi_{1}\right)\left[\sqrt{2}\left(\xi_{1}-2-\sqrt{2}\right) \sqrt{1+\xi_{2}}+(2+\sqrt{2})\left(1+\xi_{2}\right)\right] \\
& N_{d}^{6}\left(\xi_{1}, \xi_{2}\right)=\frac{\sqrt{2}}{2}\left(1-\xi_{1}^{2}\right) \sqrt{1+\xi_{2}} \\
& N_{d}^{7}\left(\xi_{1}, \xi_{2}\right)=\frac{1}{4}\left(1-\xi_{1}\right)\left[-\sqrt{2}\left(\xi_{1}+2+\sqrt{2}\right) \sqrt{1+\xi_{2}}+(2+\sqrt{2})\left(1+\xi_{2}\right)\right] \\
& N_{d}^{8}\left(\xi_{1}, \xi_{2}\right)=\frac{1}{2}\left(1-\xi_{1}\right)\left[(2+\sqrt{2}) \sqrt{1+\xi_{2}}-(1+\sqrt{2})\left(1+\xi_{2}\right)\right]
\end{aligned}
$$

$$
\begin{aligned}
& N_{t}^{1}\left(\xi_{1}, \xi_{2}\right)=N_{d}^{1}\left(\xi_{1}, \xi_{2}\right) / \sqrt{1+\xi_{2}} \\
& N_{t}^{2}\left(\xi_{1}, \xi_{2}\right)=N_{d}^{2}\left(\xi_{1}, \xi_{2}\right) / \sqrt{1+\xi_{2}} \\
& N_{t}^{3}\left(\xi_{1}, \xi_{2}\right)=N_{d}^{3}\left(\xi_{1}, \xi_{2}\right) / \sqrt{1+\xi_{2}} \\
& N_{t}^{4}\left(\xi_{1}, \xi_{2}\right)=N_{d}^{4}\left(\xi_{1}, \xi_{2}\right) / \sqrt{1+\xi_{2}} \\
& N_{t}^{5}\left(\xi_{1}, \xi_{2}\right)=\sqrt{2} N_{d}^{5}\left(\xi_{1}, \xi_{2}\right) / \sqrt{1+\xi_{2}} \\
& N_{t}^{6}\left(\xi_{1}, \xi_{2}\right)=\sqrt{2} N_{d}^{6}\left(\xi_{1}, \xi_{2}\right) / \sqrt{1+\xi_{2}} \\
& N_{t}^{7}\left(\xi_{1}, \xi_{2}\right)=\sqrt{2} N_{d}^{7}\left(\xi_{1}, \xi_{2}\right) / \sqrt{1+\xi_{2}} \\
& N_{t}^{8}\left(\xi_{1}, \xi_{2}\right)=N_{d}^{8}\left(\xi_{1}, \xi_{2}\right) / \sqrt{1+\xi_{2}}
\end{aligned}
$$


If the nodes 3-4-5 are on the crack-front, the shape functions are as follows

$$
\begin{aligned}
& N_{d}^{1}\left(\xi_{1}, \xi_{2}\right)=\frac{1}{4}\left(1-\xi_{2}\right)\left[-\sqrt{2}\left(\xi_{2}+2+\sqrt{2}\right) \sqrt{1-\xi_{1}}+(2+\sqrt{2})\left(1-\xi_{1}\right)\right] \\
& N_{d}^{2}\left(\xi_{1}, \xi_{2}\right)=\frac{1}{2}\left(1-\xi_{2}\right)\left[(2+\sqrt{2}) \sqrt{1-\xi_{1}}-(1+\sqrt{2})\left(1-\xi_{1}\right)\right] \\
& N_{d}^{3}\left(\xi_{1}, \xi_{2}\right)=\frac{1}{2}\left(1-\xi_{2}\right)\left[-\xi_{2}-\left(1-\frac{\sqrt{2}}{2} \xi_{2}\right) \sqrt{1-\xi_{1}}+\frac{\sqrt{2}}{2}\left(1-\xi_{1}\right)\right] \\
& N_{d}^{4}\left(\xi_{1}, \xi_{2}\right)=\left(1-\xi_{2}^{2}\right)\left[1-\frac{\sqrt{2}}{2} \sqrt{1-\xi_{1}}\right] \\
& N_{d}^{5}\left(\xi_{1}, \xi_{2}\right)=\frac{1}{2}\left(1+\xi_{2}\right)\left[\xi_{2}-\left(1+\frac{\sqrt{2}}{2} \xi_{2}\right) \sqrt{1-\xi_{1}}+\frac{\sqrt{2}}{2}\left(1-\xi_{1}\right)\right] \\
& N_{d}^{6}\left(\xi_{1}, \xi_{2}\right)=\frac{1}{2}\left(1+\xi_{2}\right)\left[(2+\sqrt{2}) \sqrt{1-\xi_{1}}-(1+\sqrt{2})\left(1-\xi_{1}\right)\right] \\
& N_{d}^{7}\left(\xi_{1}, \xi_{2}\right)=\frac{1}{4}\left(1+\xi_{2}\right)\left[\sqrt{2}\left(\xi_{2}-2-\sqrt{2}\right) \sqrt{1-\xi_{1}}+(2+\sqrt{2})\left(1-\xi_{1}\right)\right] \\
& N_{d}^{8}\left(\xi_{1}, \xi_{2}\right)=\frac{\sqrt{2}}{2}\left(1-\xi_{2}^{2}\right) \sqrt{1-\xi_{1}}
\end{aligned}
$$

$$
\begin{aligned}
& N_{t}^{1}\left(\xi_{1}, \xi_{2}\right)=\sqrt{2} N_{d}^{1}\left(\xi_{1}, \xi_{2}\right) / \sqrt{1-\xi_{1}} \\
& N_{t}^{2}\left(\xi_{1}, \xi_{2}\right)=N_{d}^{2}\left(\xi_{1}, \xi_{2}\right) / \sqrt{1-\xi_{1}} \\
& N_{t}^{3}\left(\xi_{1}, \xi_{2}\right)=N_{d}^{3}\left(\xi_{1}, \xi_{2}\right) / \sqrt{1-\xi_{1}} \\
& N_{t}^{4}\left(\xi_{1}, \xi_{2}\right)=N_{d}^{4}\left(\xi_{1}, \xi_{2}\right) / \sqrt{1-\xi_{1}} \\
& N_{t}^{5}\left(\xi_{1}, \xi_{2}\right)=N_{d}^{5}\left(\xi_{1}, \xi_{2}\right) / \sqrt{1-\xi_{1}} \\
& N_{t}^{6}\left(\xi_{1}, \xi_{2}\right)=N_{d}^{6}\left(\xi_{1}, \xi_{2}\right) / \sqrt{1-\xi_{1}} \\
& N_{t}^{7}\left(\xi_{1}, \xi_{2}\right)=\sqrt{2} N_{d}^{7}\left(\xi_{1}, \xi_{2}\right) / \sqrt{1-\xi_{1}} \\
& N_{t}^{8}\left(\xi_{1}, \xi_{2}\right)=\sqrt{2} N_{d}^{8}\left(\xi_{1}, \xi_{2}\right) / \sqrt{1-\xi_{1}}
\end{aligned}
$$


If the nodes 5-6-7 are on the crack-front, the shape functions are

$$
\begin{aligned}
& N_{d}^{1}\left(\xi_{1}, \xi_{2}\right)=\frac{1}{4}\left(1-\xi_{1}\right)\left[-\sqrt{2}\left(\xi_{1}+2+\sqrt{2}\right) \sqrt{1-\xi_{1}}+(2+\sqrt{2})\left(1-\xi_{2}\right)\right] \\
& N_{d}^{2}\left(\xi_{1}, \xi_{2}\right)=\frac{\sqrt{2}}{2}\left(1-\xi_{1}^{2}\right) \sqrt{1-\xi_{2}} \\
& N_{d}^{3}\left(\xi_{1}, \xi_{2}\right)=\frac{1}{4}\left(1+\xi_{1}\right)\left[\sqrt{2}\left(\xi_{1}-2-\sqrt{2}\right) \sqrt{1-\xi_{2}}+(2+\sqrt{2})\left(1-\xi_{2}\right)\right] \\
& N_{d}^{4}\left(\xi_{1}, \xi_{2}\right)=\frac{1}{2}\left(1+\xi_{1}\right)\left[(2+\sqrt{2}) \sqrt{1-\xi_{2}}-(1+\sqrt{2})\left(1-\xi_{2}\right)\right] \\
& N_{d}^{5}\left(\xi_{1}, \xi_{2}\right)=\frac{1}{2}\left(1+\xi_{1}\right)\left[\xi_{1}-\left(1+\frac{\sqrt{2}}{2} \xi_{1}\right) \sqrt{1-\xi_{2}}+\frac{\sqrt{2}}{2}\left(1-\xi_{2}\right)\right] \\
& N_{d}^{6}\left(\xi_{1}, \xi_{2}\right)=\left(1-\xi_{1}^{2}\right)\left[1-\frac{\sqrt{2}}{2} \sqrt{1-\xi_{2}}\right] \\
& N_{d}^{7}\left(\xi_{1}, \xi_{2}\right)=\frac{1}{2}\left(1-\xi_{1}\right)\left[-\xi_{1}-\left(1-\frac{\sqrt{2}}{2} \xi_{1}\right) \sqrt{1-\xi_{2}}+\frac{\sqrt{2}}{2}\left(1-\xi_{2}\right)\right] \\
& N_{d}^{8}\left(\xi_{1}, \xi_{2}\right)=\frac{1}{2}\left(1-\xi_{1}\right)\left[(2+\sqrt{2}) \sqrt{1-\xi_{2}}-(1+\sqrt{2})\left(1-\xi_{2}\right)\right]
\end{aligned}
$$

$$
\begin{aligned}
& N_{t}^{1}\left(\xi_{1}, \xi_{2}\right)=\sqrt{2} N_{d}^{1}\left(\xi_{1}, \xi_{2}\right) / \sqrt{1-\xi_{2}} \\
& N_{t}^{2}\left(\xi_{1}, \xi_{2}\right)=\sqrt{2} N_{d}^{2}\left(\xi_{1}, \xi_{2}\right) / \sqrt{1-\xi_{2}} \\
& N_{t}^{3}\left(\xi_{1}, \xi_{2}\right)=\sqrt{2} N_{d}^{3}\left(\xi_{1}, \xi_{2}\right) / \sqrt{1-\xi_{2}} \\
& N_{t}^{4}\left(\xi_{1}, \xi_{2}\right)=N_{d}^{4}\left(\xi_{1}, \xi_{2}\right) / \sqrt{1-\xi_{2}} \\
& N_{t}^{5}\left(\xi_{1}, \xi_{2}\right)=N_{d}^{5}\left(\xi_{1}, \xi_{2}\right) / \sqrt{1-\xi_{2}} \\
& N_{t}^{6}\left(\xi_{1}, \xi_{2}\right)=N_{d}^{6}\left(\xi_{1}, \xi_{2}\right) / \sqrt{1-\xi_{2}} \\
& N_{t}^{7}\left(\xi_{1}, \xi_{2}\right)=N_{d}^{7}\left(\xi_{1}, \xi_{2}\right) / \sqrt{1-\xi_{2}} \\
& N_{t}^{8}\left(\xi_{1}, \xi_{2}\right)=N_{d}^{8}\left(\xi_{1}, \xi_{2}\right) / \sqrt{1-\xi_{2}}
\end{aligned}
$$


If the nodes 7-8-1 are on the crack-front, the shape functions are

$$
\begin{aligned}
& N_{d}^{1}\left(\xi_{1}, \xi_{2}\right)=\frac{1}{2}\left(1-\xi_{2}\right)\left[-\xi_{2}-\left(1-\frac{\sqrt{2}}{2} \xi_{2}\right) \sqrt{1+\xi_{1}}+\frac{\sqrt{2}}{2}\left(1+\xi_{1}\right)\right] \\
& N_{d}^{2}\left(\xi_{1}, \xi_{2}\right)=\frac{1}{2}\left(1-\xi_{2}\right)\left[(2+\sqrt{2}) \sqrt{1+\xi_{1}}-(1+\sqrt{2})\left(1+\xi_{1}\right)\right] \\
& N_{d}^{3}\left(\xi_{1}, \xi_{2}\right)=\frac{1}{4}\left(1-\xi_{2}\right)\left[-\sqrt{2}\left(\xi_{2}+2+\sqrt{2}\right) \sqrt{1+\xi_{1}}+(2+\sqrt{2})\left(1+\xi_{1}\right)\right] \\
& N_{d}^{4}\left(\xi_{1}, \xi_{2}\right)=\frac{\sqrt{2}}{2}\left(1-\xi_{2}^{2}\right) \sqrt{1+\xi_{1}} \\
& N_{d}^{5}\left(\xi_{1}, \xi_{2}\right)=\frac{1}{4}\left(1+\xi_{2}\right)\left[\sqrt{2}\left(\xi_{2}-2-\sqrt{2}\right) \sqrt{1+\xi_{1}}+(2+\sqrt{2})\left(1+\xi_{1}\right)\right] \\
& N_{d}^{6}\left(\xi_{1}, \xi_{2}\right)=\frac{1}{2}\left(1+\xi_{2}\right)\left[(2+\sqrt{2}) \sqrt{1+\xi_{1}}-(1+\sqrt{2})\left(1+\xi_{1}\right)\right] \\
& N_{d}^{7}\left(\xi_{1}, \xi_{2}\right)=\frac{1}{2}\left(1+\xi_{2}\right)\left[\xi_{2}-\left(1+\frac{\sqrt{2}}{2} \xi_{2}\right) \sqrt{1+\xi_{1}}+\frac{\sqrt{2}}{2}\left(1+\xi_{1}\right)\right] \\
& N_{d}^{8}\left(\xi_{1}, \xi_{2}\right)=\left(1-\xi_{2}^{2}\right)\left[1-\frac{\sqrt{2}}{2} \sqrt{1+\xi_{1}}\right]
\end{aligned}
$$

$$
\begin{aligned}
& N_{t}^{1}\left(\xi_{1}, \xi_{2}\right)=N_{d}^{1}\left(\xi_{1}, \xi_{2}\right) / \sqrt{1+\xi_{1}} \\
& N_{t}^{2}\left(\xi_{1}, \xi_{2}\right)=N_{d}^{2}\left(\xi_{1}, \xi_{2}\right) / \sqrt{1+\xi_{1}} \\
& N_{t}^{3}\left(\xi_{1}, \xi_{2}\right)=\sqrt{2} N_{d}^{3}\left(\xi_{1}, \xi_{2}\right) / \sqrt{1+\xi_{1}} \\
& N_{t}^{4}\left(\xi_{1}, \xi_{2}\right)=\sqrt{2} N_{d}^{4}\left(\xi_{1}, \xi_{2}\right) / \sqrt{1+\xi_{1}} \\
& N_{t}^{5}\left(\xi_{1}, \xi_{2}\right)=\sqrt{2} N_{d}^{5}\left(\xi_{1}, \xi_{2}\right) / \sqrt{1+\xi_{1}} \\
& N_{t}^{6}\left(\xi_{1}, \xi_{2}\right)=N_{d}^{6}\left(\xi_{1}, \xi_{2}\right) / \sqrt{1+\xi_{1}} \\
& N_{t}^{7}\left(\xi_{1}, \xi_{2}\right)=N_{d}^{7}\left(\xi_{1}, \xi_{2}\right) / \sqrt{1+\xi_{1}} \\
& N_{t}^{8}\left(\xi_{1}, \xi_{2}\right)=N_{d}^{8}\left(\xi_{1}, \xi_{2}\right) / \sqrt{1+\xi_{1}}
\end{aligned}
$$


Appendix B

Stress Intensity Factors for a Crack in a Pipe Bend 


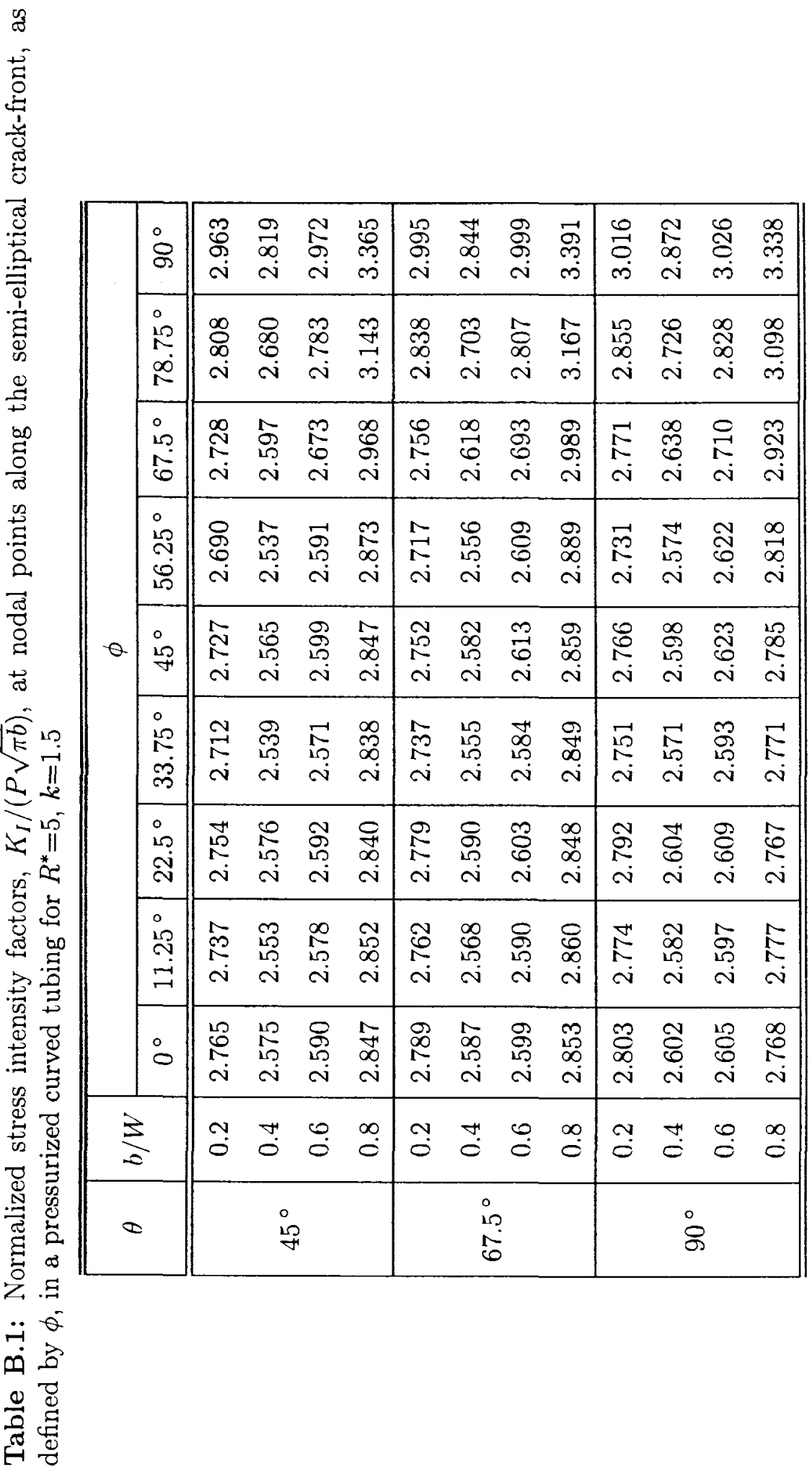




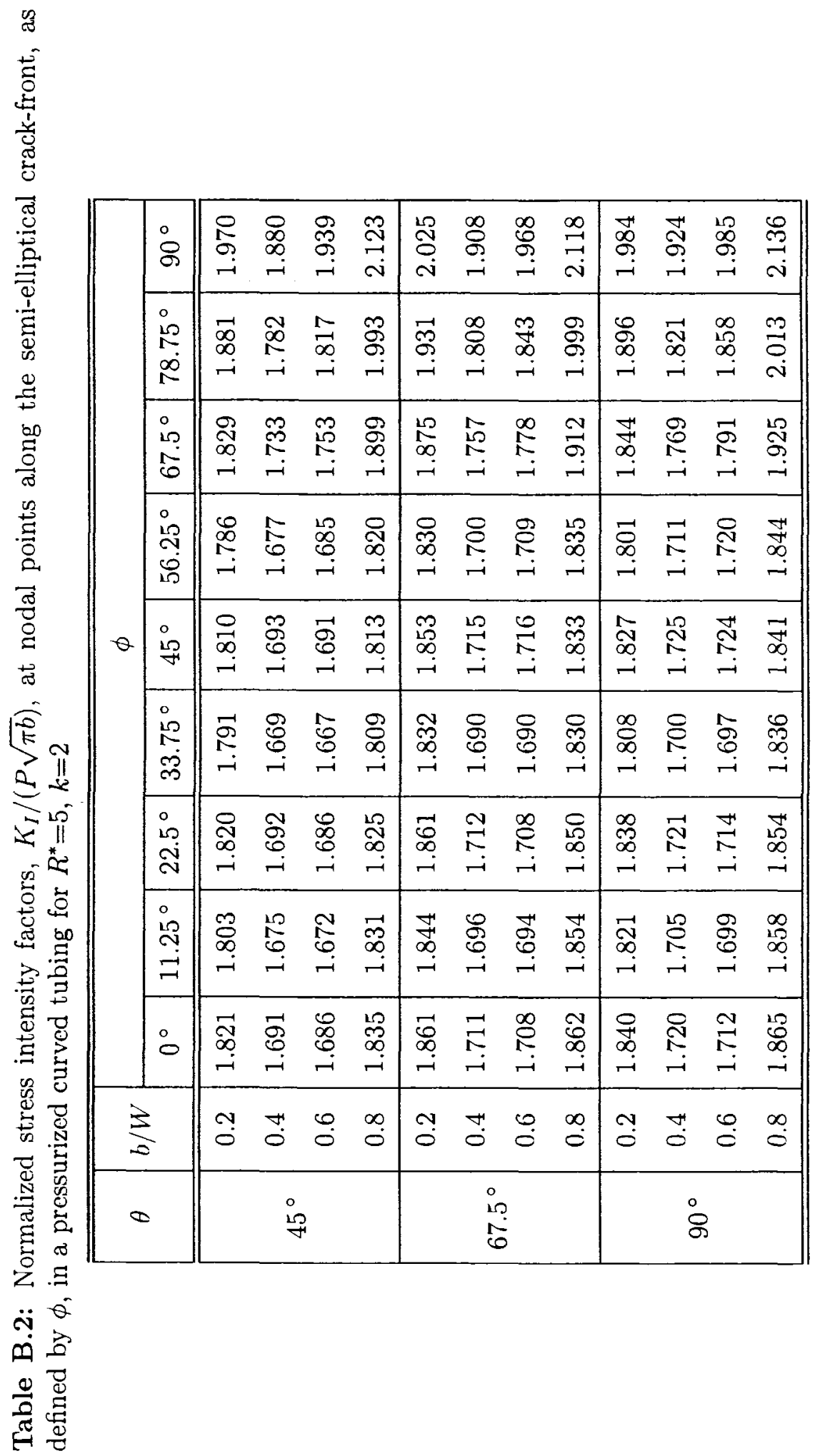




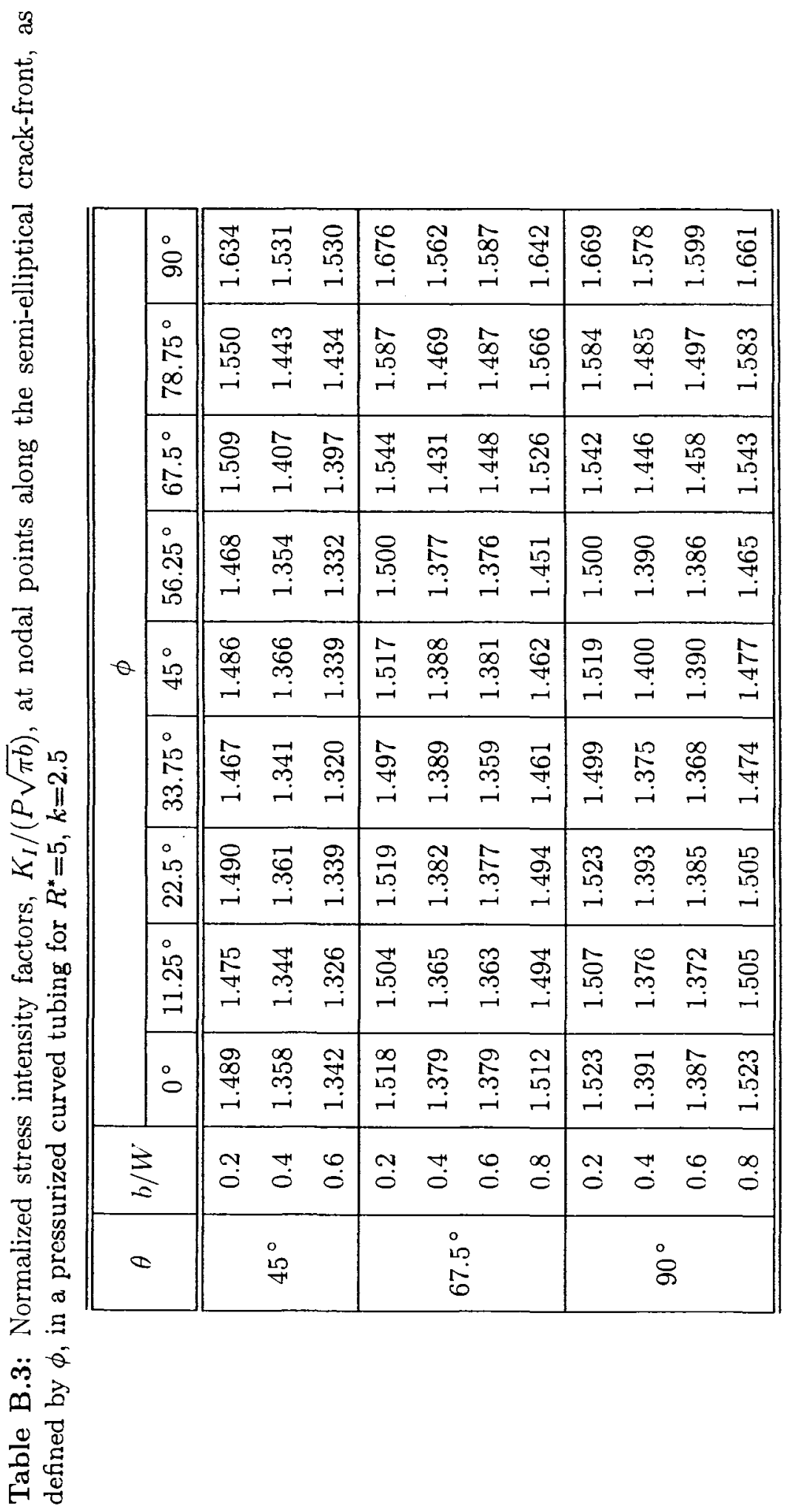




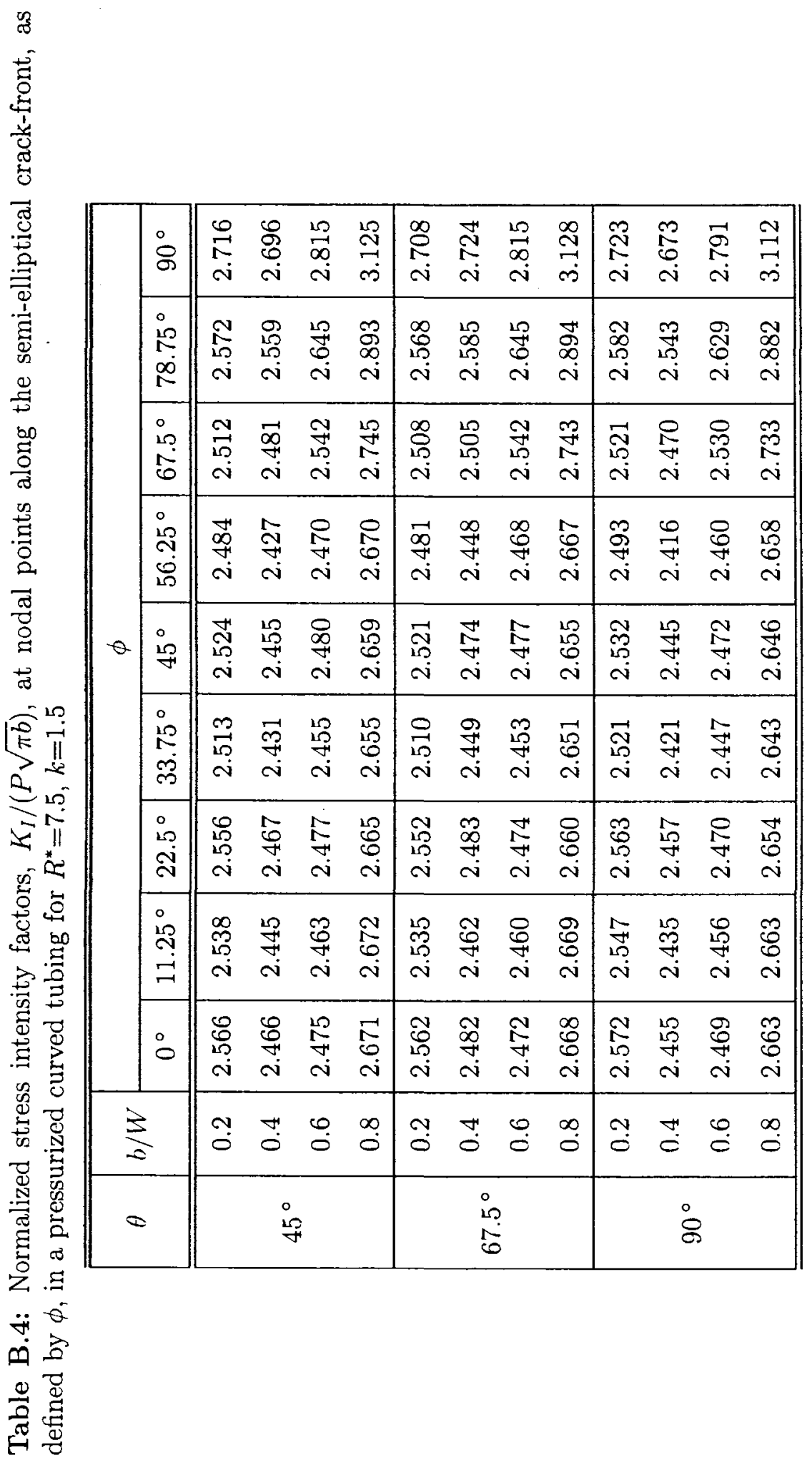




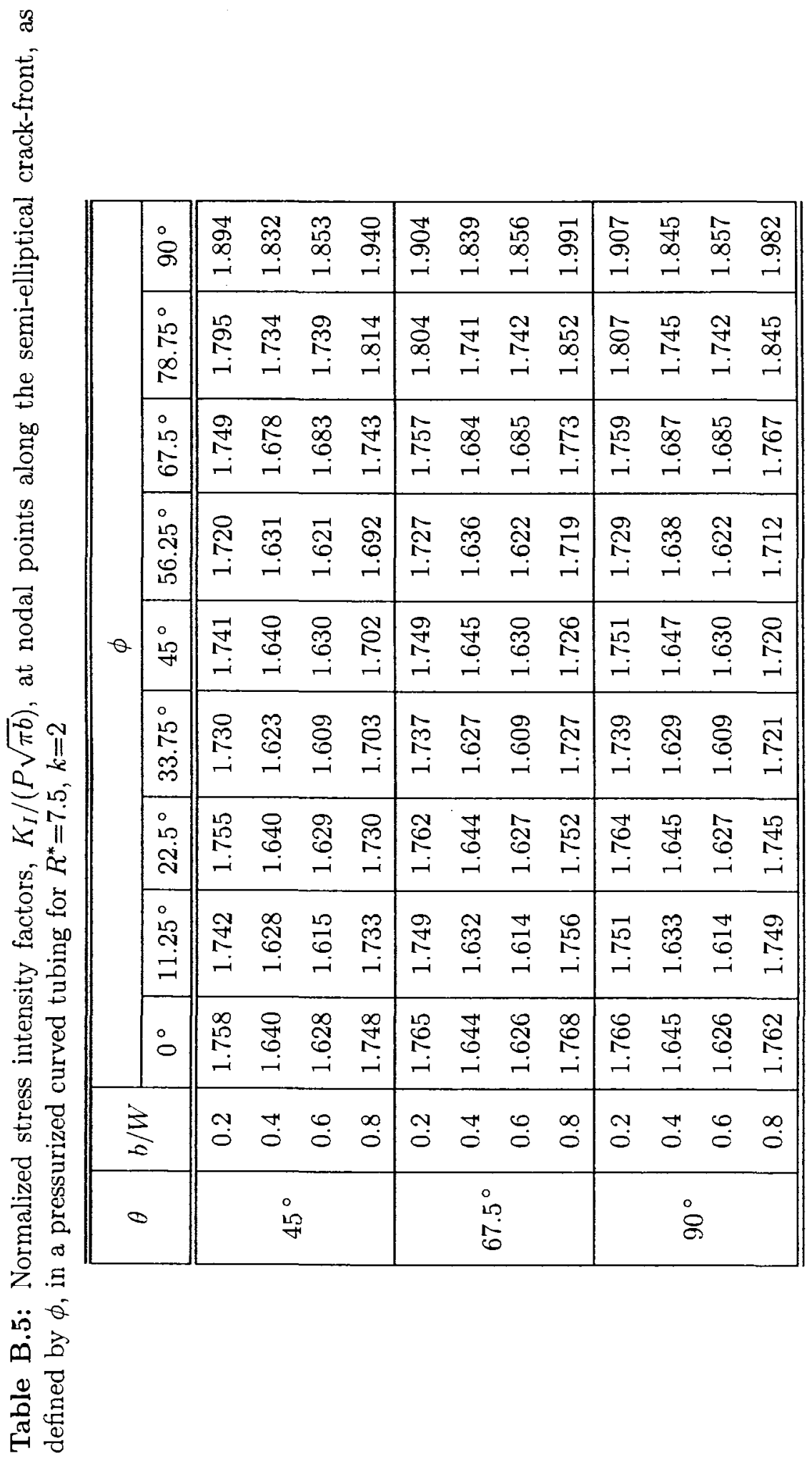




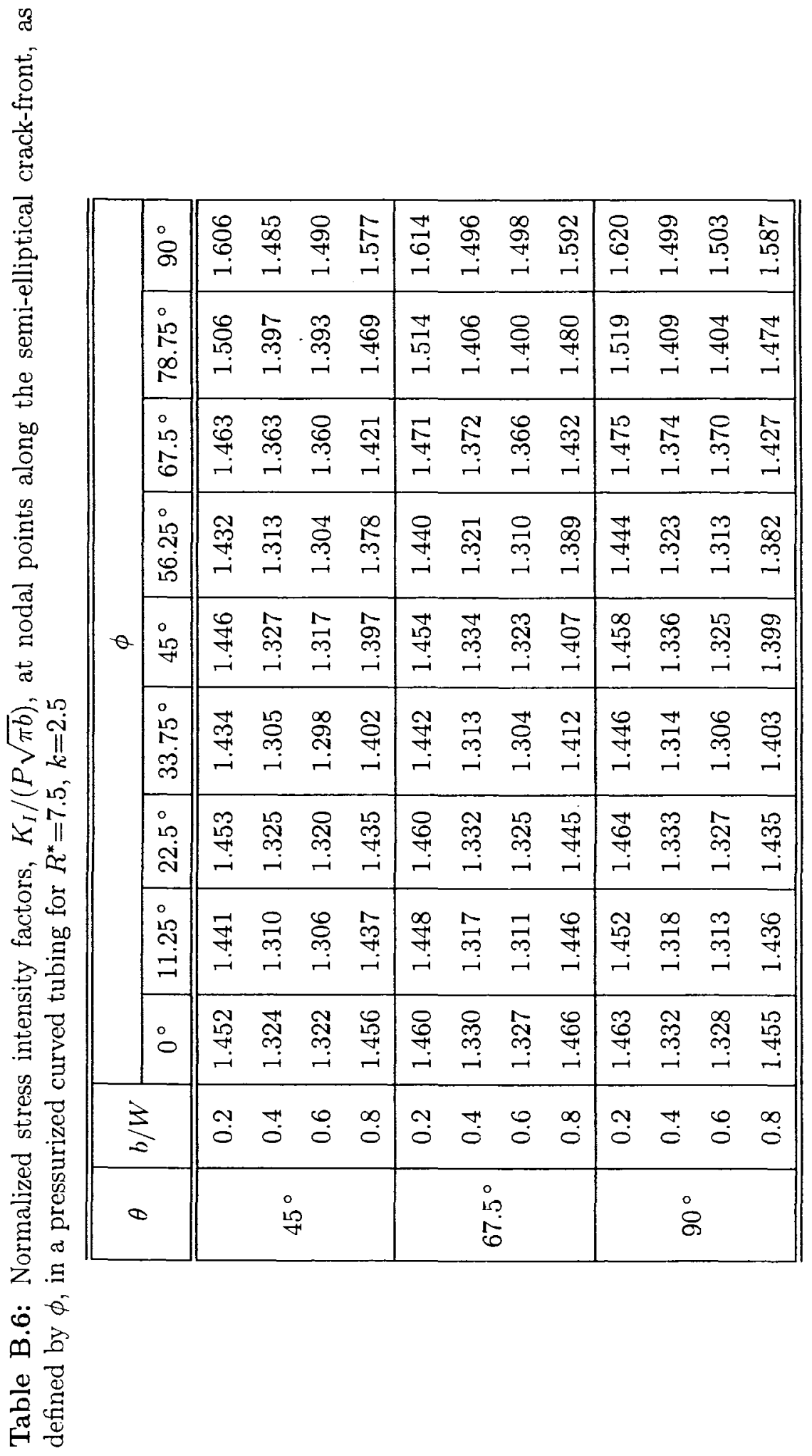




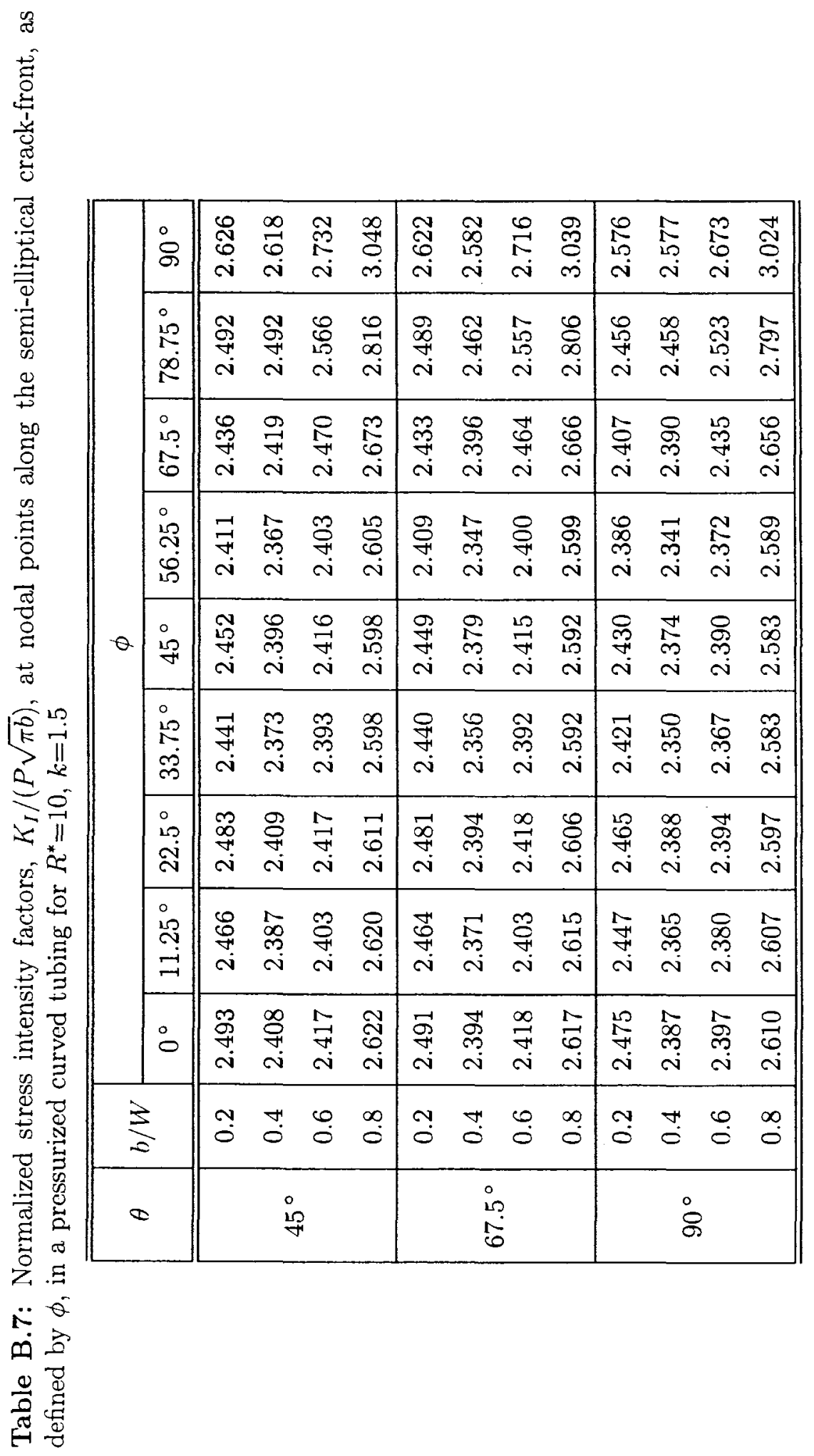




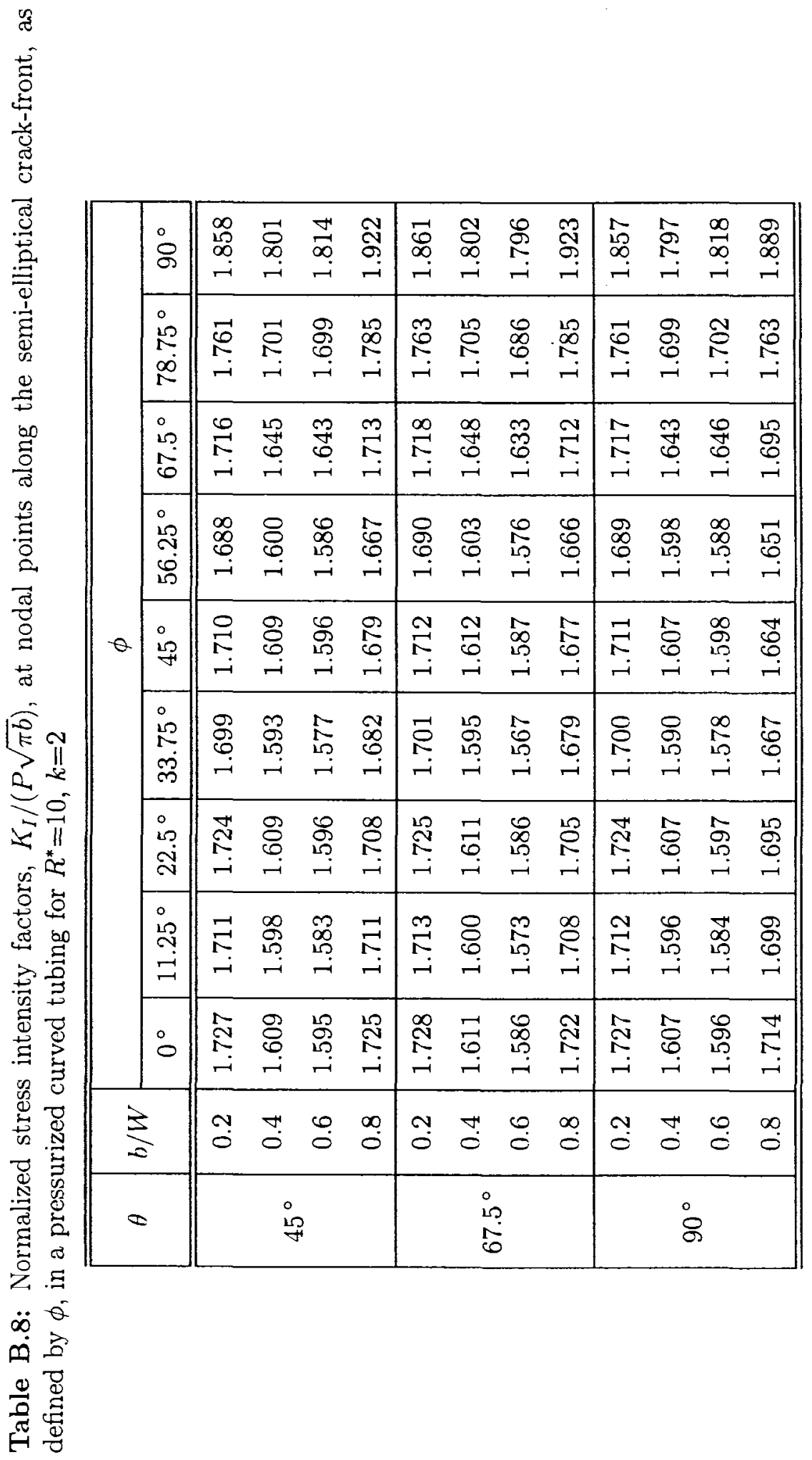




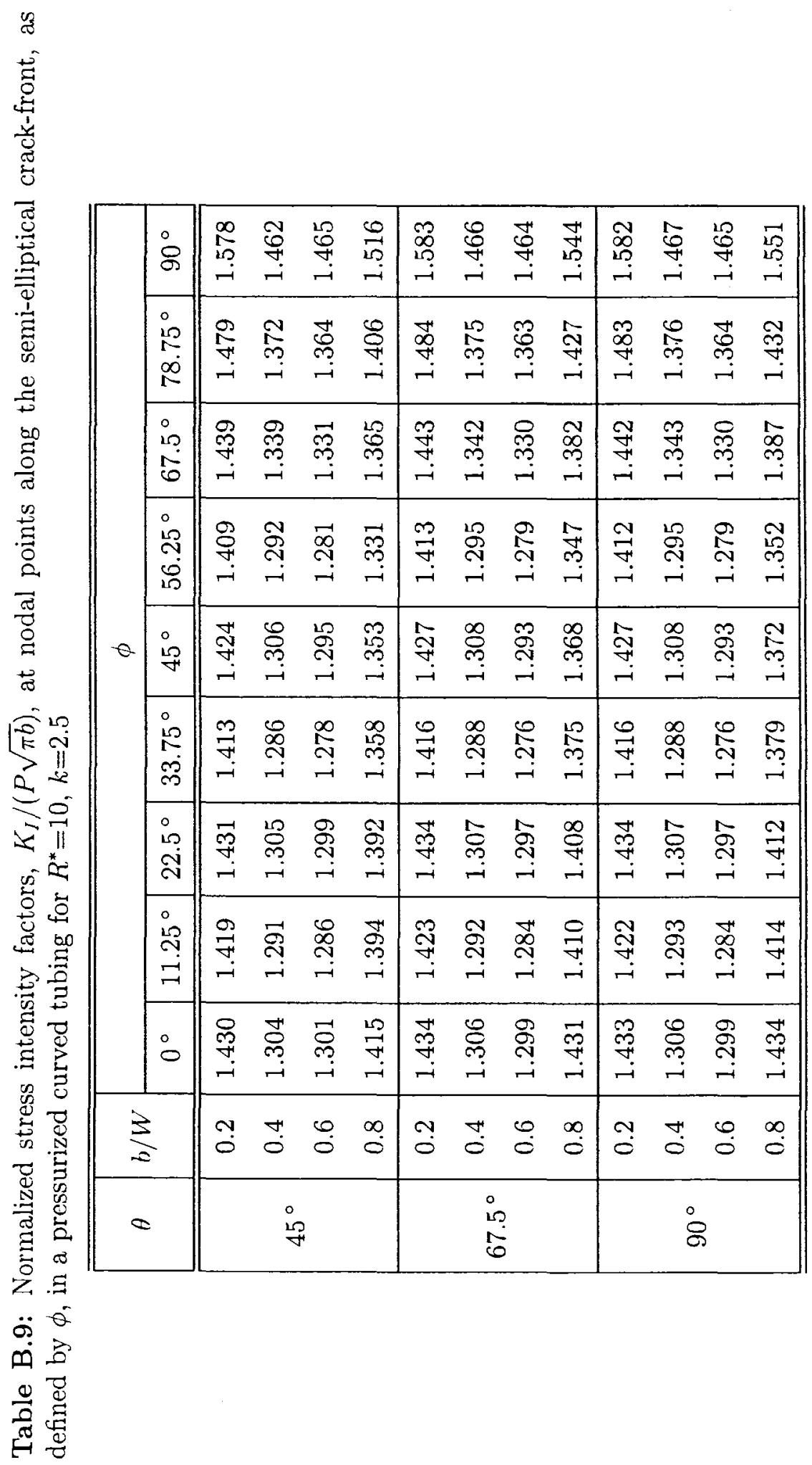


Appendix C

Influence Coefficients and Stress Intensity

Factors for a Crack in a Pipe Bend

C.1 Influence Coefficients 


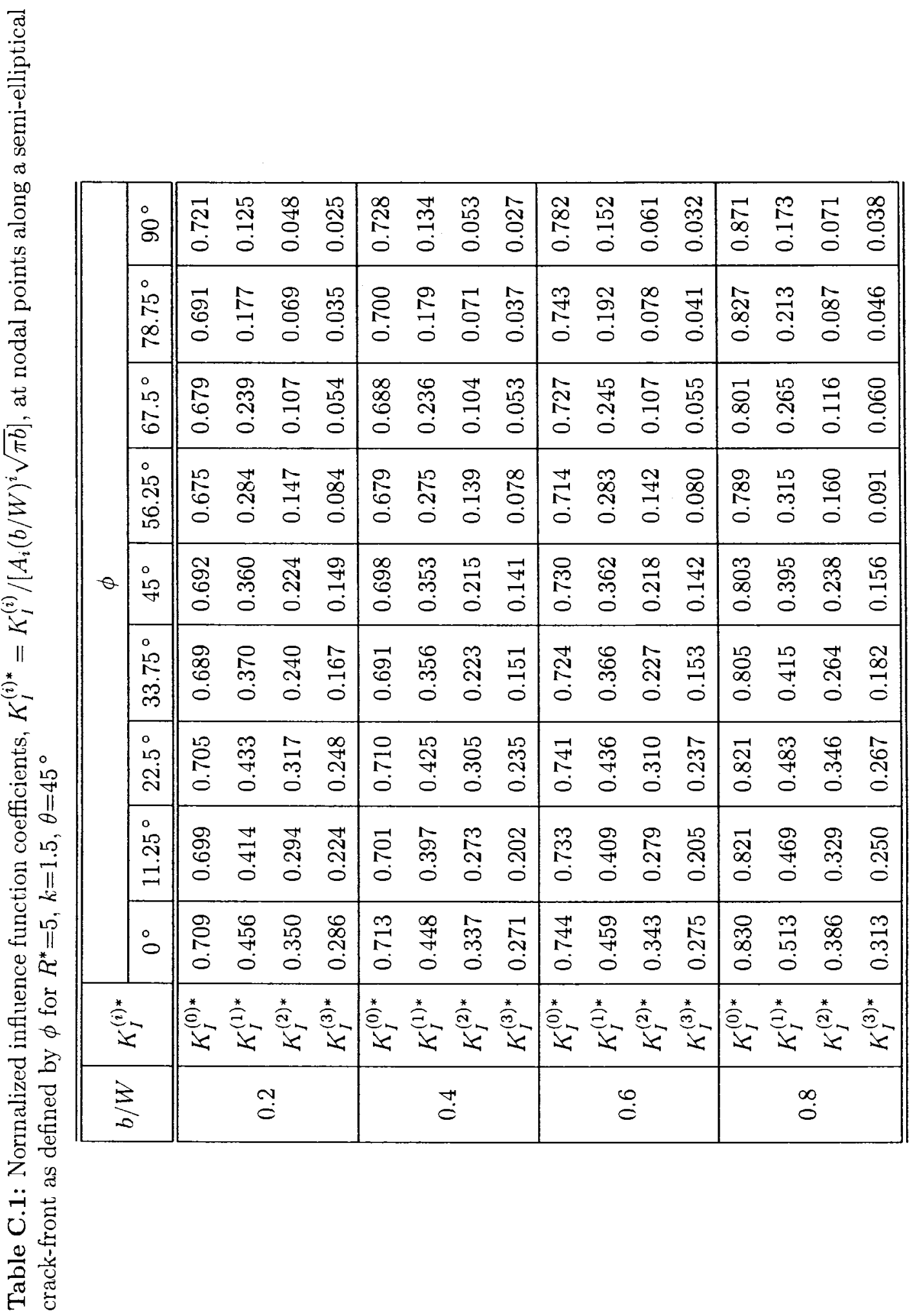




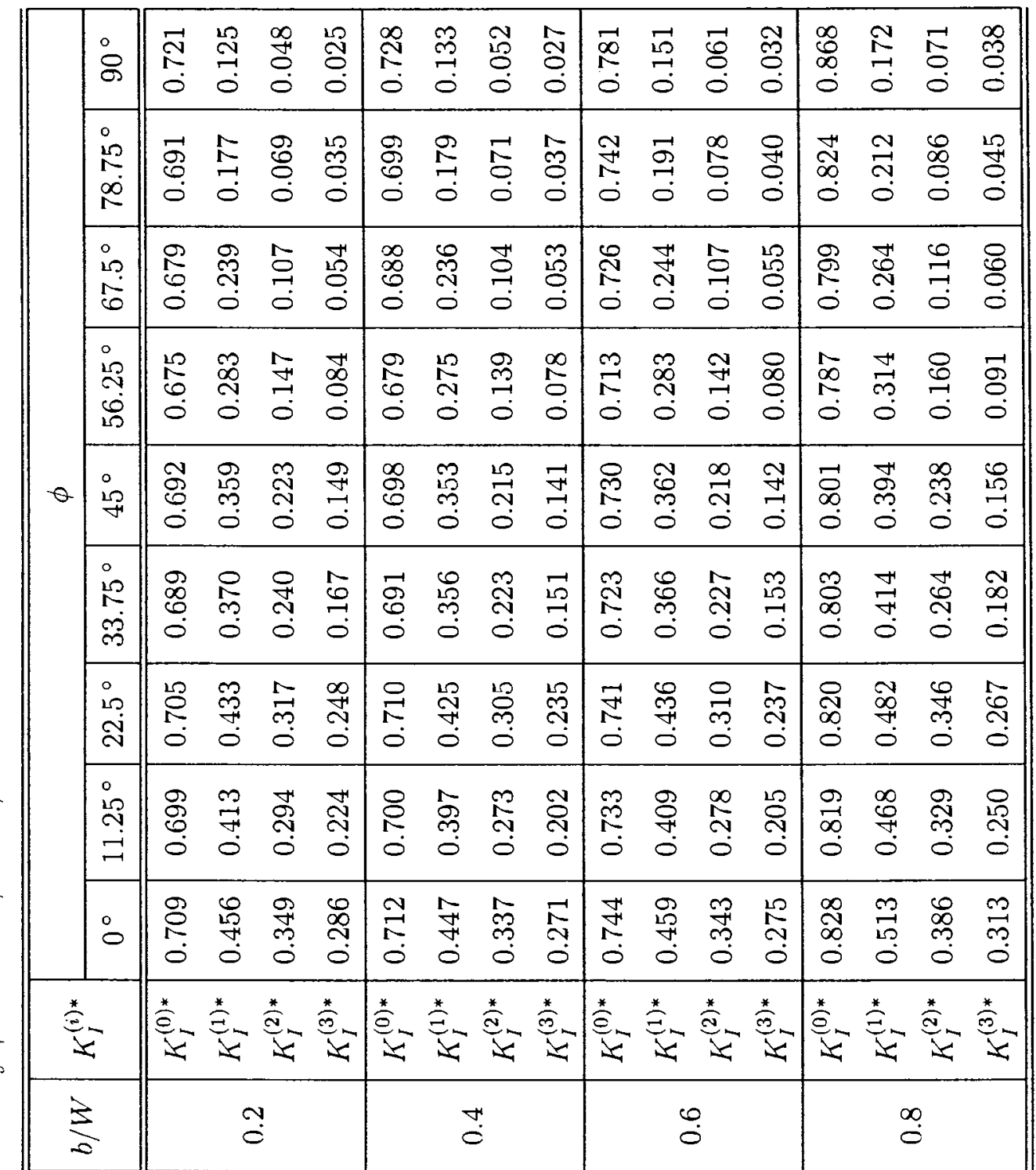

乙

نे

离 


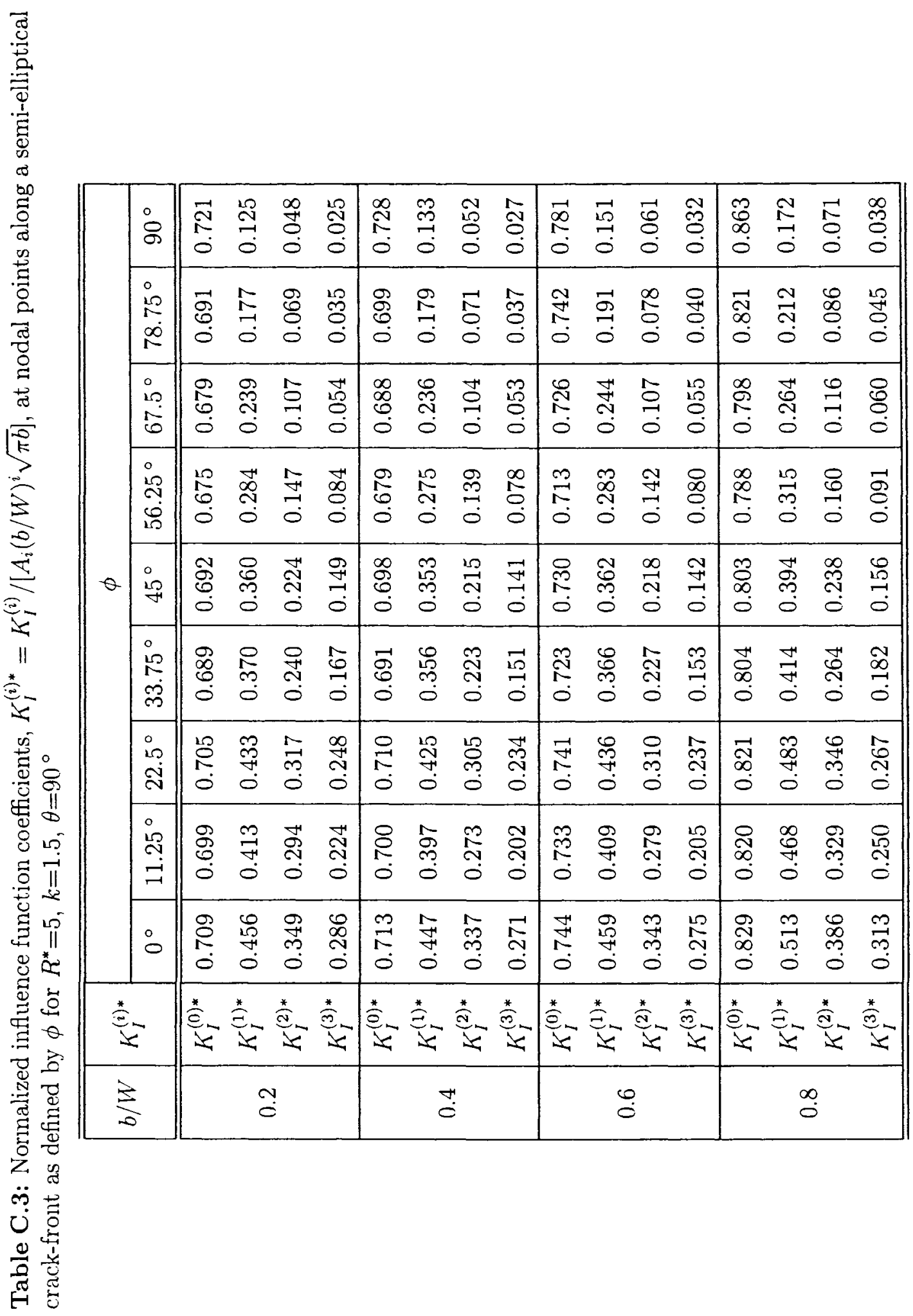




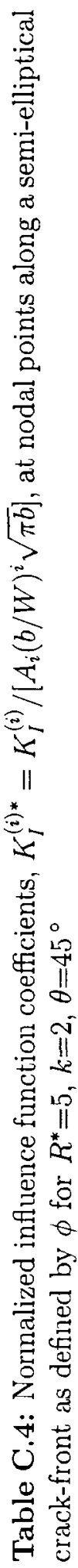

\begin{tabular}{|c|c|c|c|c|c|c|c|c|c|c|c|c|c|c|c|c|c|}
\hline & $\stackrel{\circ}{8}$ & $\begin{array}{l}\mathscr{M} \\
0 \\
0 \\
0\end{array}$ & $\stackrel{g}{\vec{Z}}$ & $\begin{array}{l}\mathscr{H} \\
\stackrel{્}{0}\end{array}$ & 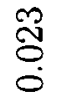 & $\begin{array}{l}\mathscr{0} \\
0 \\
0 \\
0\end{array}$ & $\underset{\stackrel{\sim}{\sim}}{\stackrel{\sim}{0}}$ & 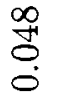 & 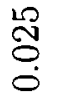 & 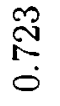 & $\stackrel{\text { mे }}{\overrightarrow{0}}$ & $\begin{array}{l}0 \\
\stackrel{1}{0} \\
0 \\
0\end{array}$ & 尽 & $\begin{array}{l}5 \\
\infty \\
\infty \\
0\end{array}$ & $\begin{array}{l}\stackrel{\leftrightarrow}{0} \\
\stackrel{0}{0}\end{array}$ & $\begin{array}{l}\infty \\
\stackrel{8}{0}\end{array}$ & 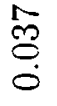 \\
\hline & $\begin{array}{l}\frac{1}{2} \\
\stackrel{\infty}{1} \\
1\end{array}$ & $\begin{array}{l}\mathscr{8} \\
\stackrel{0}{0} \\
0\end{array}$ & $\begin{array}{l}\text { Do } \\
\stackrel{-}{0}\end{array}$ & $\begin{array}{l}2 \\
8 \\
0 \\
0\end{array}$ & $\begin{array}{l}\text { m } \\
\stackrel{0}{0}\end{array}$ & $\begin{array}{l}\stackrel{\leftrightarrow}{ } \\
\stackrel{\leftrightarrow}{0}\end{array}$ & $\begin{array}{l}\stackrel{0}{0} \\
\stackrel{1}{0}\end{array}$ & $\stackrel{\mathscr{8}}{\mathscr{8}}$ & 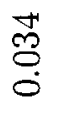 & $\begin{array}{l}\infty \\
\stackrel{8}{0} \\
\stackrel{0}{0}\end{array}$ & $\begin{array}{l}0 \\
\stackrel{1}{0} \\
0\end{array}$ & $\stackrel{\vec{O}}{\circ}$ & 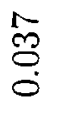 & $\begin{array}{l}\frac{2}{\infty} \\
\stackrel{0}{0} \\
0\end{array}$ & $\begin{array}{l}\frac{9}{7} \\
\stackrel{0}{0}\end{array}$ & $\begin{array}{l}\stackrel{\infty}{0} \\
\stackrel{0}{0}\end{array}$ & $\stackrel{?}{\stackrel{\Xi}{巳 巳 ~}}$ \\
\hline & is & $\begin{array}{l}0 \\
0 \\
0 \\
0\end{array}$ & 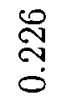 & $\begin{array}{l}\stackrel{8}{8} \\
\stackrel{8}{0} \\
0\end{array}$ & $\begin{array}{l}\stackrel{8}{\circ} \\
\stackrel{0}{0}\end{array}$ & $\begin{array}{l}\mathscr{O} \\
\stackrel{0}{0} \\
0\end{array}$ & $\begin{array}{l}\text { ని } \\
0\end{array}$ & $\begin{array}{l}\vec{S} \\
\stackrel{8}{0}\end{array}$ & 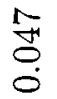 & $\begin{array}{l}10 \\
\mathbb{8} \\
0 \\
0\end{array}$ & 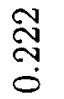 & $\begin{array}{l}\vec{S} \\
\stackrel{8}{0}\end{array}$ & 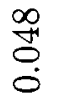 & $\underset{⿱ N}{N}$ & 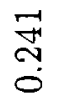 & $\begin{array}{l}\stackrel{S}{O} \\
\stackrel{0}{0}\end{array}$ & $\begin{array}{l}\text { Iึ } \\
\stackrel{8}{0} \\
0\end{array}$ \\
\hline & $\begin{array}{l}10 \\
\stackrel{10}{0} \\
0 \\
10\end{array}$ & 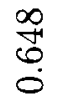 & 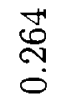 & $\begin{array}{l}\vec{m} \\
\stackrel{m}{0} \\
0\end{array}$ & $\begin{array}{l}10 \\
0 \\
0 \\
0\end{array}$ & $\begin{array}{l}\overrightarrow{5} \\
\stackrel{0}{0} \\
0\end{array}$ & 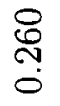 & $\stackrel{\text { Фి }}{\stackrel{\oplus}{\circ}}$ & $\begin{array}{l}\mathbb{N} \\
0 \\
0\end{array}$ & \begin{tabular}{l}
\multirow{\infty}{\infty}{} \\
0 \\
$\stackrel{0}{0}$
\end{tabular} & 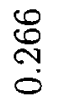 & $\begin{array}{l}\vec{m} \\
\stackrel{0}{0}\end{array}$ & 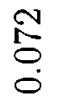 & $\begin{array}{l}\infty \\
\stackrel{0}{0} \\
0\end{array}$ & 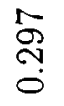 & $\begin{array}{l}\stackrel{0}{+} \\
\stackrel{4}{0}\end{array}$ & $\begin{array}{l}\vec{\infty} \\
\stackrel{0}{0} \\
0\end{array}$ \\
\hline$\theta$ & $\begin{array}{l}19 \\
19\end{array}$ & $\begin{array}{l}\mathscr{8} \\
\stackrel{8}{0} \\
0\end{array}$ & 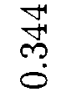 & $\begin{array}{l}0 \\
\stackrel{0}{0} \\
0\end{array}$ & 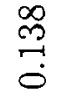 & $\begin{array}{l}\stackrel{N}{0} \\
0 \\
0\end{array}$ & 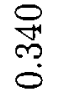 & \begin{tabular}{l}
$\stackrel{2}{8}$ \\
\hdashline \\
0
\end{tabular} & $\stackrel{\mathscr{m}}{\dddot{m}}$ & $\begin{array}{l}\stackrel{0}{\Gamma} \\
\stackrel{0}{0}\end{array}$ & 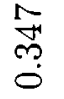 & $\begin{array}{l}20 \\
\text { กุ } \\
0\end{array}$ & $\stackrel{\vec{m}}{\overrightarrow{0}}$ & $\begin{array}{l}\frac{10}{R} \\
0 \\
0\end{array}$ & 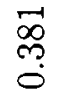 & 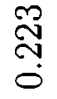 & $\underset{\stackrel{\overbrace{}}{\rightleftarrows}}{\stackrel{\rightleftarrows}{\rightleftarrows}}$ \\
\hline & $\begin{array}{l}\text { in } \\
\text { nj } \\
\text { ming }\end{array}$ & $\begin{array}{l}3 \\
\stackrel{8}{8} \\
0\end{array}$ & 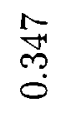 & $\begin{array}{l}\infty \\
\stackrel{\infty}{N} \\
0\end{array}$ & $\begin{array}{l}\stackrel{\infty}{+} \\
\stackrel{1}{0}\end{array}$ & $\begin{array}{l}\mathscr{\ell} \\
\mathscr{\ell} \\
0\end{array}$ & 点 & 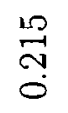 & 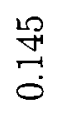 & $\begin{array}{l}\stackrel{?}{?} \\
\stackrel{0}{0}\end{array}$ & $\begin{array}{l}10 \\
10 \\
0 \\
0\end{array}$ & $\begin{array}{l}\stackrel{0}{\overrightarrow{2}} \\
\stackrel{-}{0}\end{array}$ & $\begin{array}{l}\stackrel{9}{H} \\
\stackrel{0}{0}\end{array}$ & $\begin{array}{l}\overrightarrow{0} \\
\infty \\
0 \\
0\end{array}$ & $\begin{array}{l}\infty \\
\stackrel{+}{0} \\
0\end{array}$ & 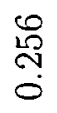 & $\stackrel{\vec{I}}{\stackrel{\Xi}{0}}$ \\
\hline & $\begin{array}{l}\text { in } \\
\text { in }\end{array}$ & $\begin{array}{l}\mathscr{M} \\
\infty \\
0 \\
0\end{array}$ & 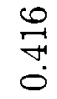 & 占 & $\begin{array}{l}\text { ్ֶ } \\
\stackrel{0}{0}\end{array}$ & $\begin{array}{l}\mathscr{0} \\
0 \\
0 \\
0\end{array}$ & $\begin{array}{l}\stackrel{0}{\nexists} \\
\stackrel{\leftrightarrow}{0}\end{array}$ & 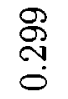 & 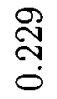 & 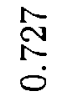 & \begin{tabular}{l}
$\stackrel{\infty}{\sharp}$ \\
\multirow{3}{*}{}
\end{tabular} & 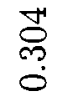 & $\begin{array}{l}\stackrel{\mathscr{乃}}{0} \\
\text { }\end{array}$ & $\begin{array}{l}\stackrel{\mathscr{Q}}{0} \\
\infty \\
0\end{array}$ & $\begin{array}{l}\text { ô } \\
\stackrel{\infty}{0} \\
0\end{array}$ & $\begin{array}{l}\underset{\text { F }}{0} \\
0\end{array}$ & $\begin{array}{l}\stackrel{\mathfrak{f}}{0} \\
0\end{array}$ \\
\hline & 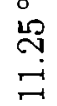 & 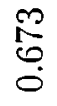 & $\begin{array}{l}\infty \\
\infty \\
\stackrel{\infty}{0} \\
0\end{array}$ & $\begin{array}{l}\infty \\
\stackrel{0}{0} \\
0\end{array}$ & $\begin{array}{l}\stackrel{\mathscr{S}}{\overrightarrow{0}} \\
\stackrel{0}{0}\end{array}$ & $\begin{array}{l}\stackrel{0}{0} \\
\stackrel{0}{0}\end{array}$ & \begin{tabular}{l}
$\infty$ \\
$\infty$ \\
$\dddot{0}$ \\
\hdashline
\end{tabular} & $\begin{array}{l}\tilde{0} \\
\dddot{0} \\
0\end{array}$ & $\begin{array}{l}\infty \\
\stackrel{\infty}{-1} \\
\sim \\
0\end{array}$ & $\stackrel{10}{\overrightarrow{0}}$ & $\underset{⿱}{\stackrel{5}{0}}$ & 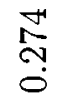 & 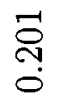 & 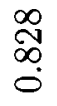 & $\stackrel{?}{\stackrel{R}{0}}$ & $\begin{array}{l}\stackrel{\infty}{N} \\
\text { } \\
ٍ\end{array}$ & 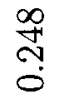 \\
\hline & $\circ$ & $\begin{array}{l}0 \\
0 \\
0 \\
0\end{array}$ & 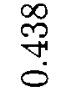 & 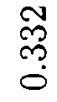 & $\begin{array}{l}\infty \\
\stackrel{\infty}{0} \\
0\end{array}$ & $\begin{array}{l}\mathscr{n} \\
\stackrel{0}{0} \\
0\end{array}$ & 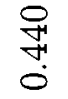 & 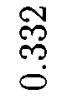 & $\begin{array}{l}\infty \\
\stackrel{\infty}{0} \\
0\end{array}$ & $\begin{array}{l}\stackrel{N}{ก} \\
0\end{array}$ & $\begin{array}{l}\stackrel{H}{5} \\
\stackrel{5}{0}\end{array}$ & 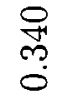 & 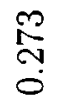 & $\begin{array}{l}\stackrel{M}{\not} \\
\infty \\
0\end{array}$ & $\begin{array}{l}10 \\
00 \\
0\end{array}$ & $\begin{array}{l}n \\
\infty \\
m \\
0\end{array}$ & $\stackrel{m}{\tilde{m}}$ \\
\hline & & $\stackrel{\text { E }}{\stackrel{*}{E}}$ & 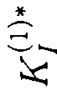 & $\bar{d}$ & $\underline{x}$ & $\stackrel{*}{\stackrel{*}{e}}$ & $\stackrel{*}{L}_{1}^{*}$ & $\frac{1}{d}$ & $\stackrel{\frac{\pi}{3}}{*}$ & 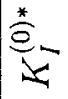 & $\stackrel{*}{\sum_{0}^{*}}$ & $\underset{\underline{S}}{\stackrel{*}{S}}$ & 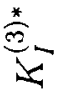 & 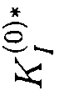 & 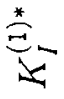 & 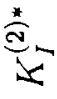 & $\stackrel{*}{\stackrel{*}{e}}$ \\
\hline & & & & & & & & & & & & & & & & & \\
\hline
\end{tabular}

4 on

8

告 


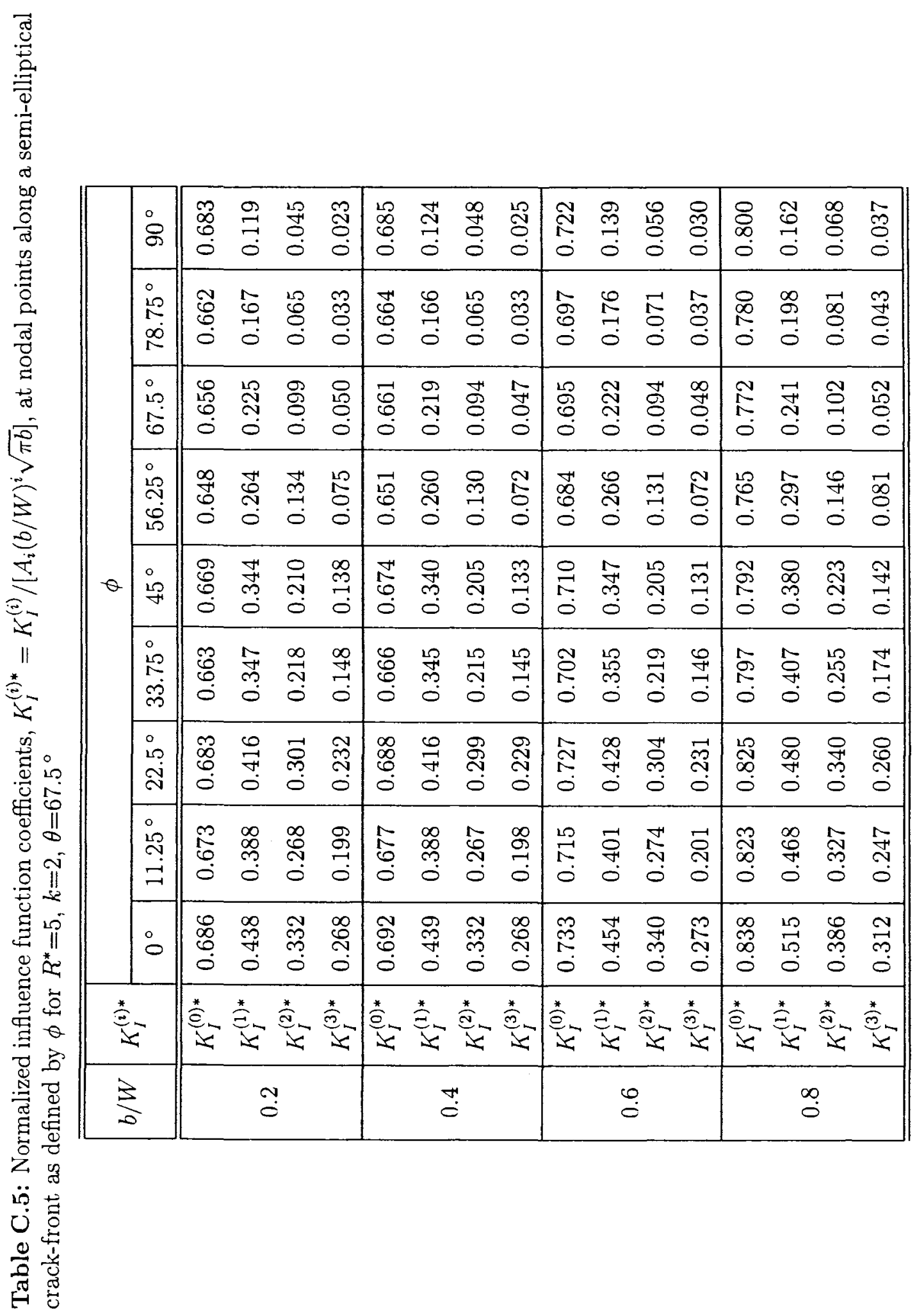




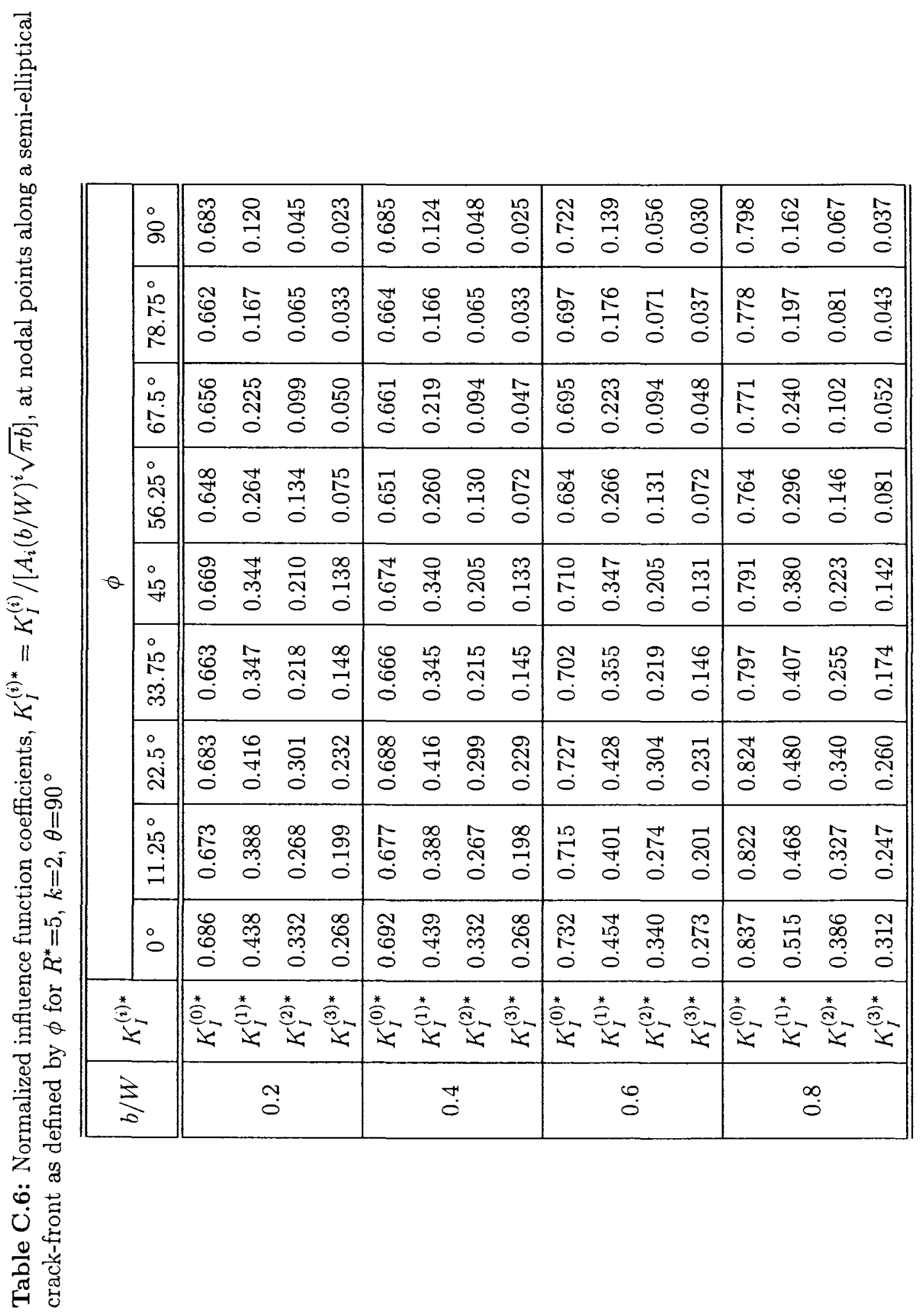




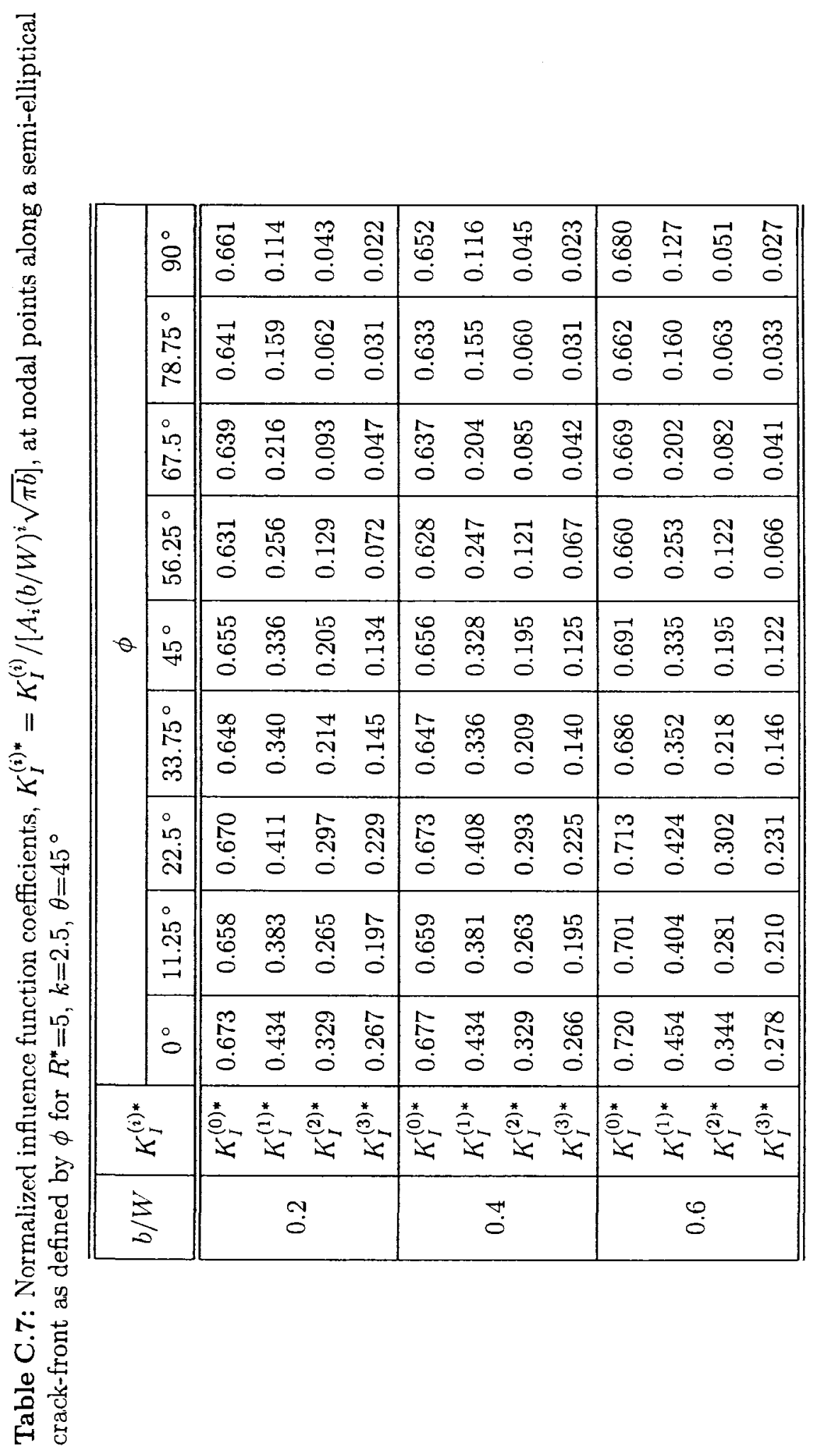




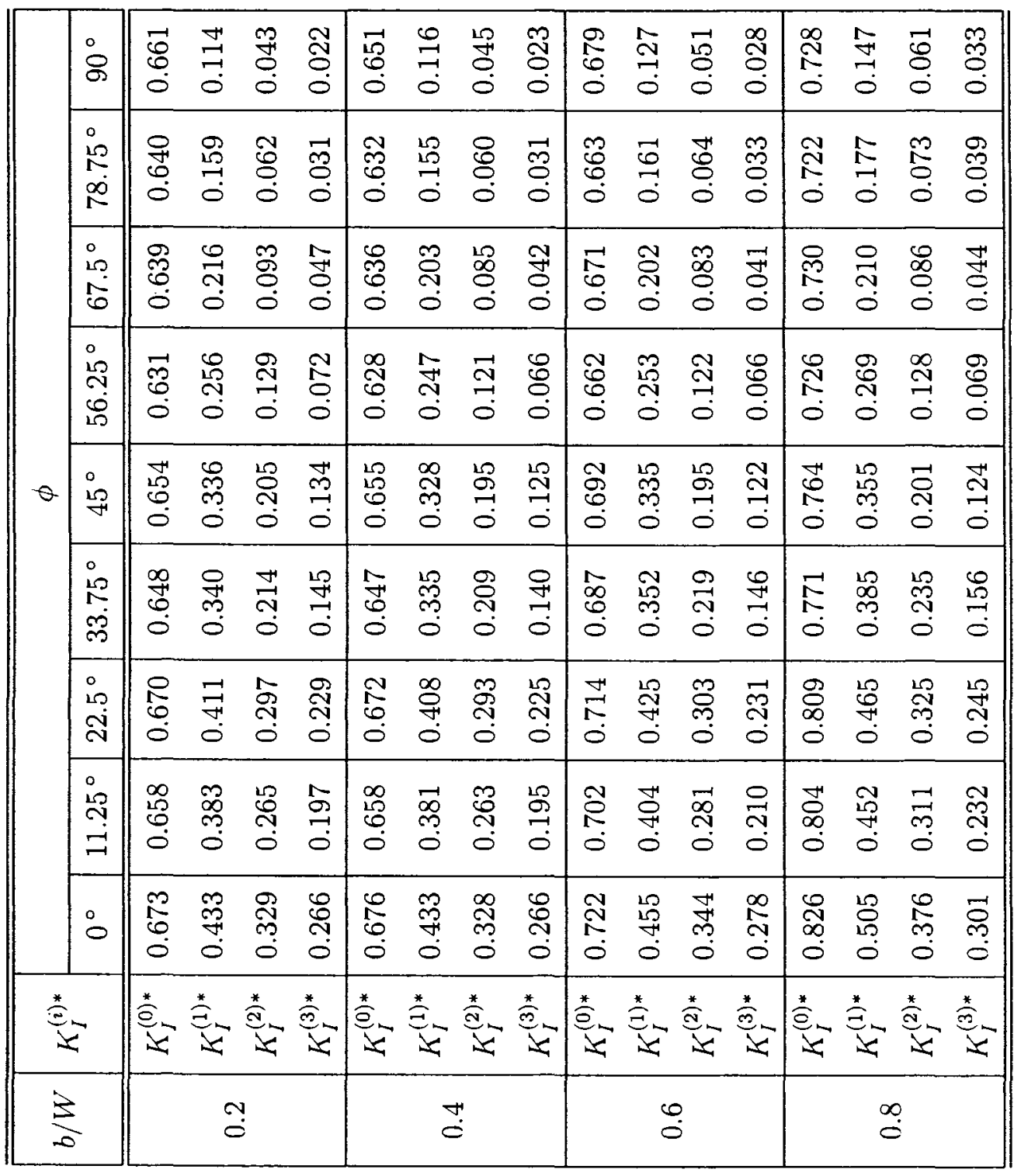

ฐิ

$\dot{0}$ 


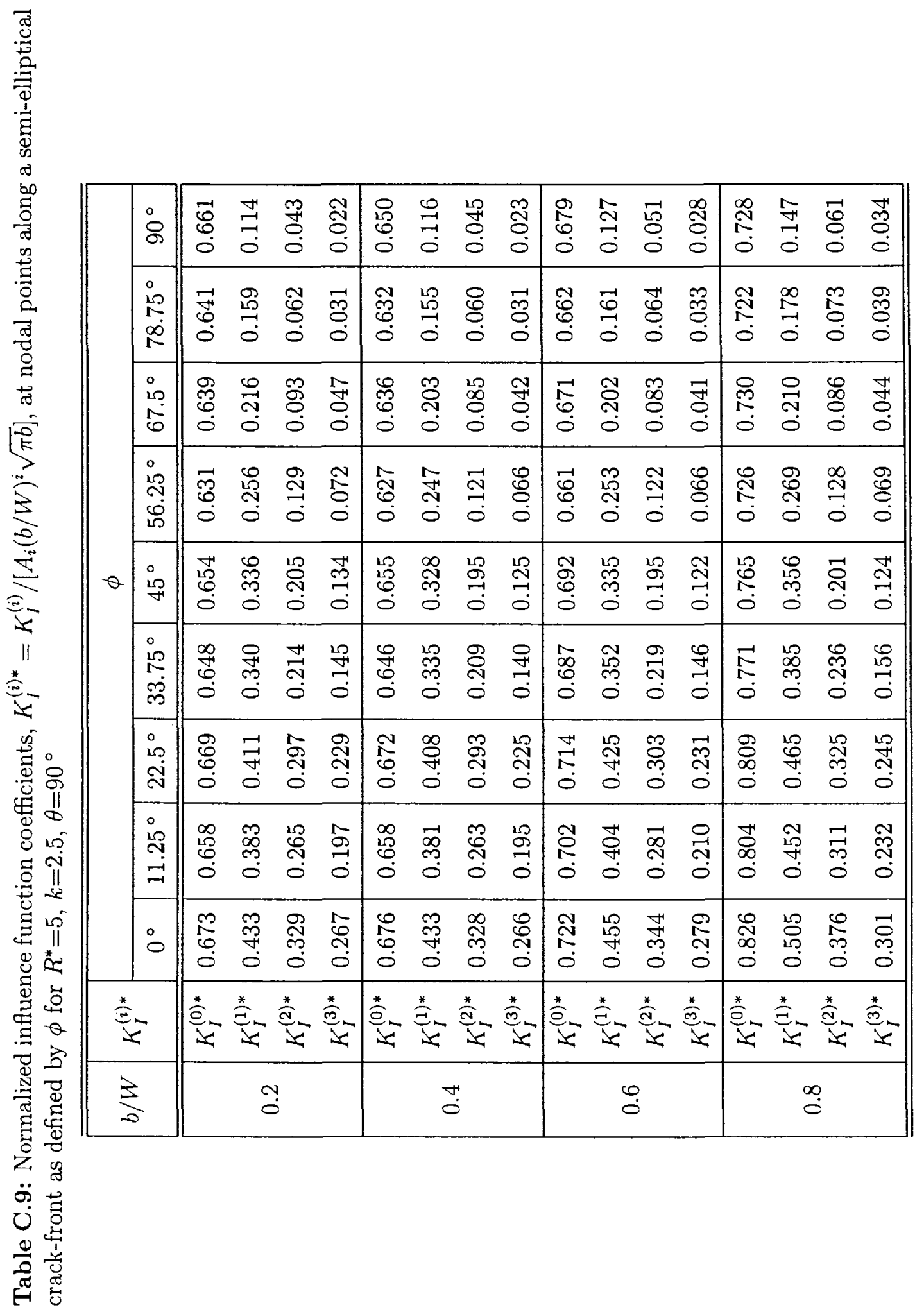




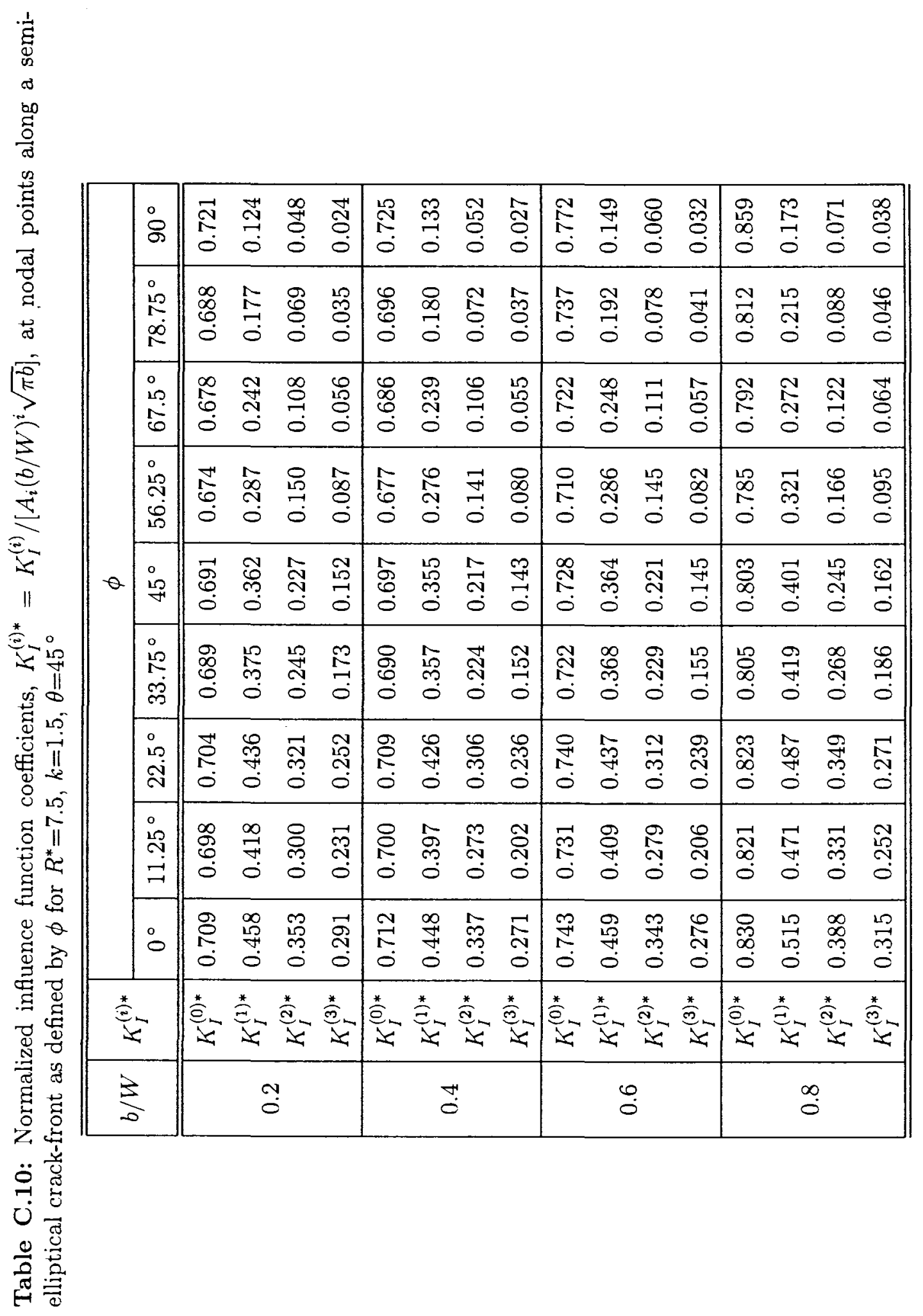




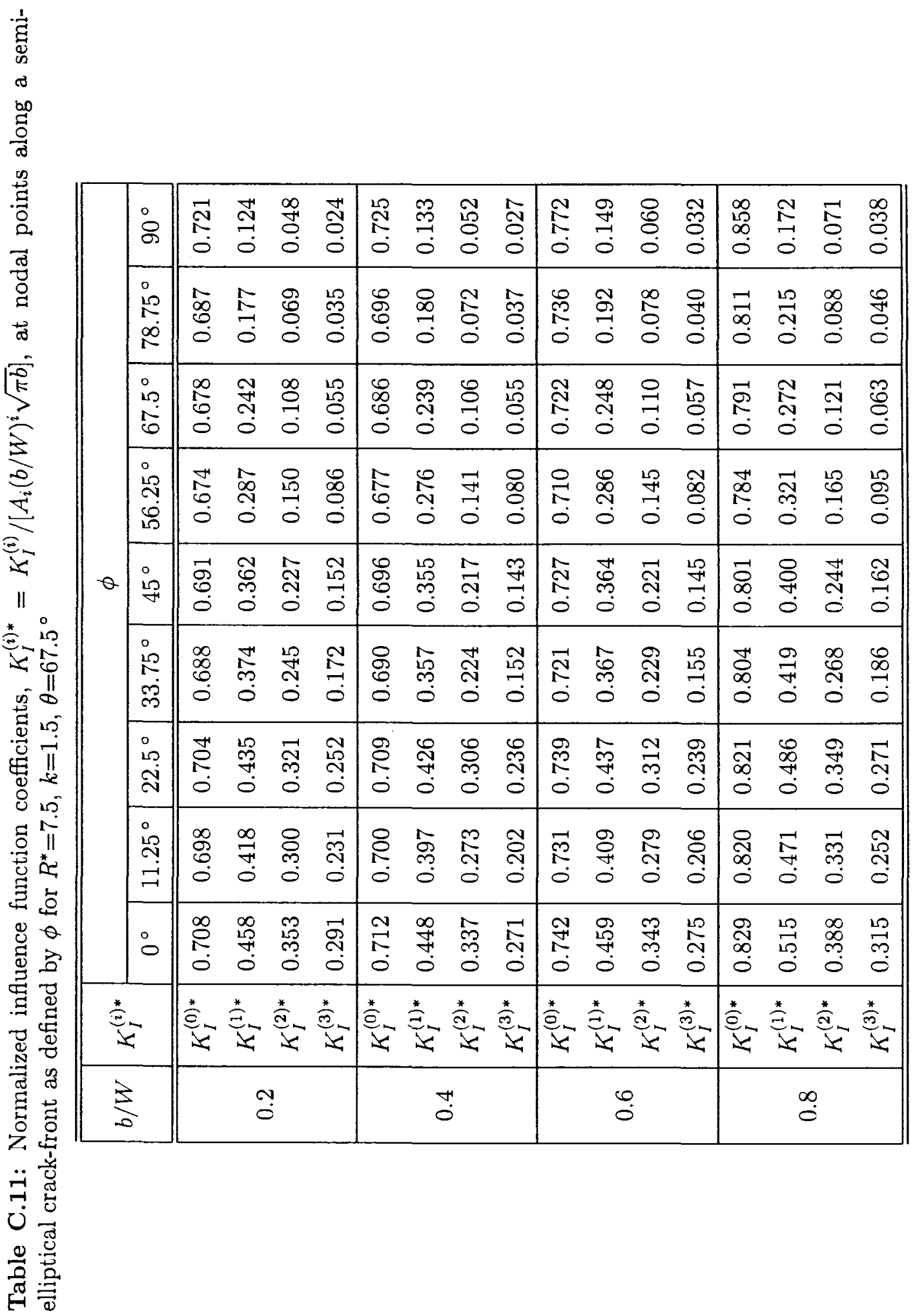




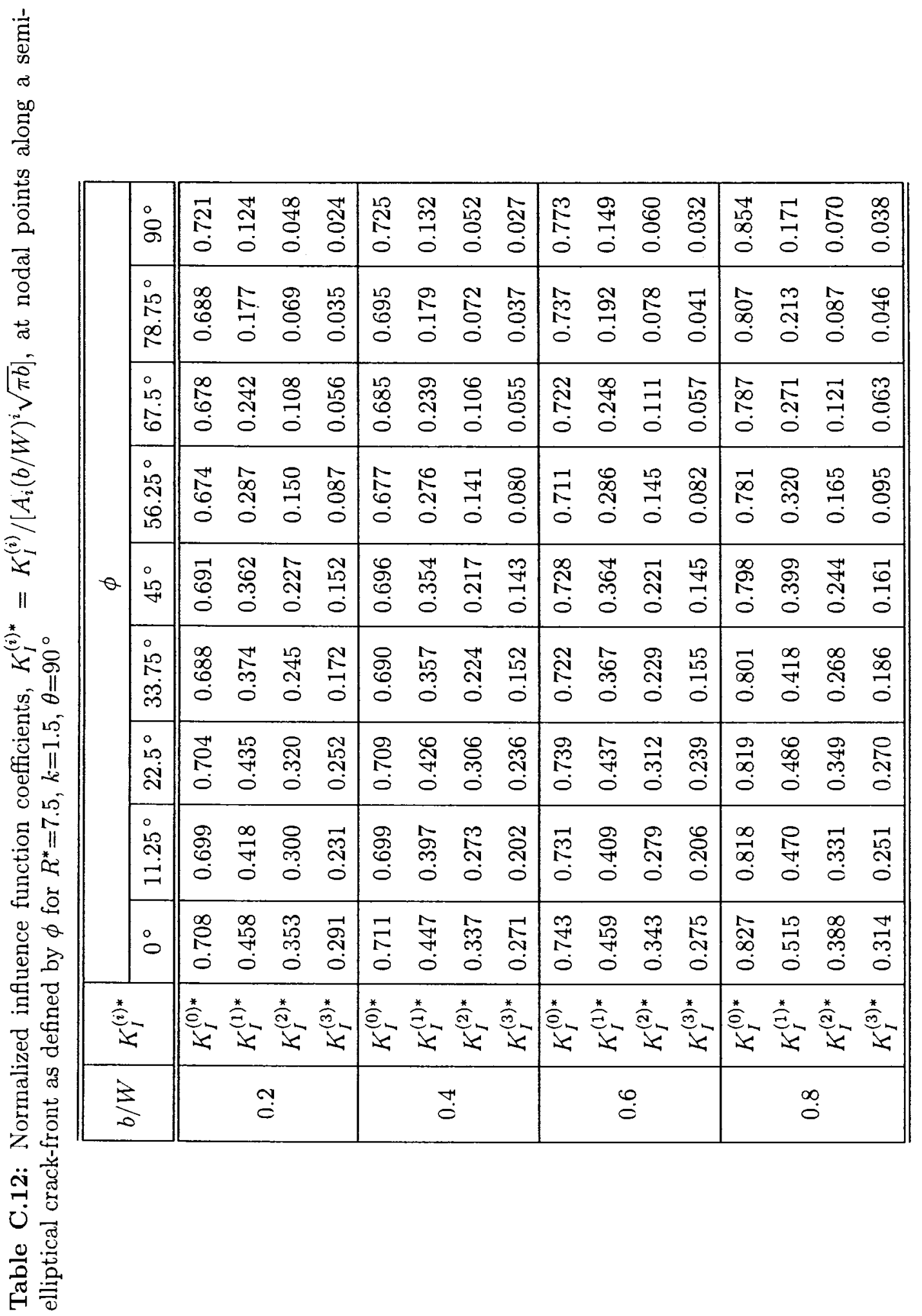


$\stackrel{*}{5}$

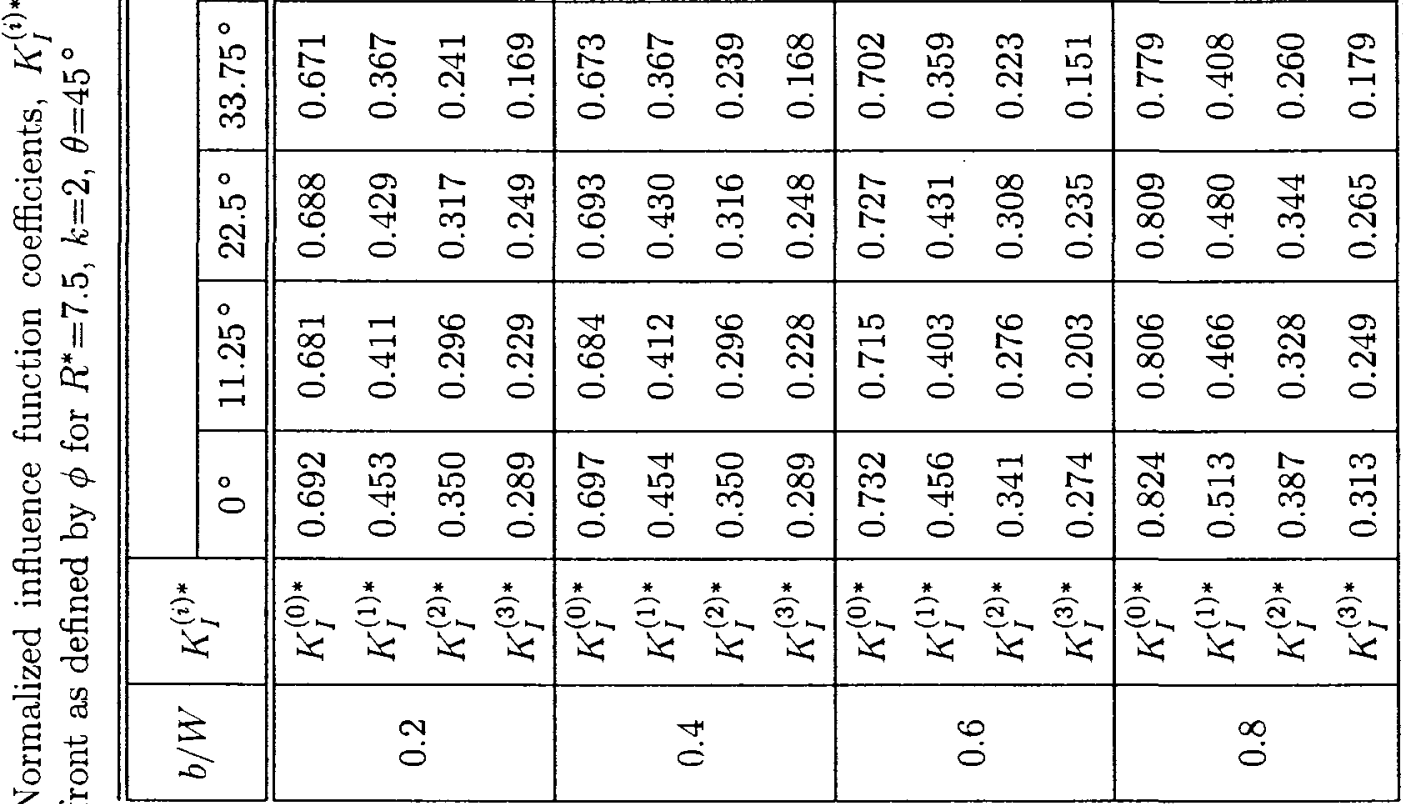

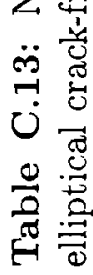




\begin{tabular}{|c|c|c|c|c|c|}
\hline \multirow{9}{*}{ 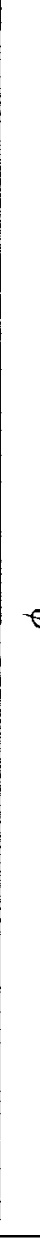 } & \& & 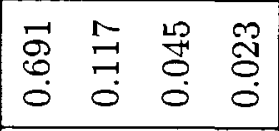 & 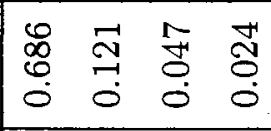 & 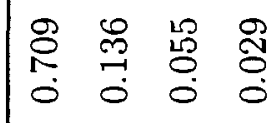 & 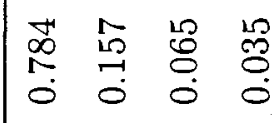 \\
\hline & 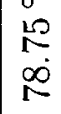 & $\begin{array}{llll}\mathbb{0} & 0 & 1 & 0 \\
0 & 0 & 0 \\
0 & 0 & 0 & 0 \\
0 & 0 & 0 & 0 \\
0\end{array}$ & $\mid \begin{array}{llll}\mathbb{Z} & \infty & 1 & 0 \\
0 & 0 & 0 & 0 \\
0 & 0 & 0 & 0 \\
0 & 0 & 0 & 0\end{array}$ & 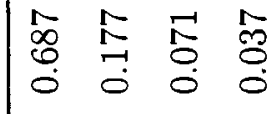 & 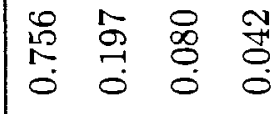 \\
\hline & 疍 & 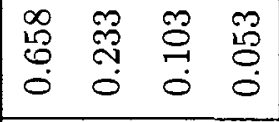 & 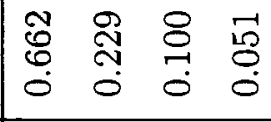 & 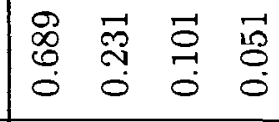 & 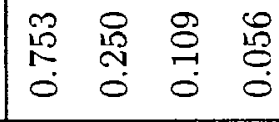 \\
\hline & 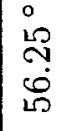 & 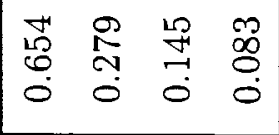 & 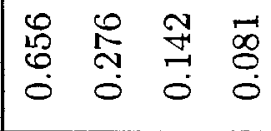 & 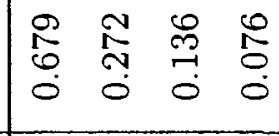 & 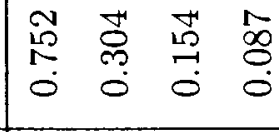 \\
\hline & 总 & 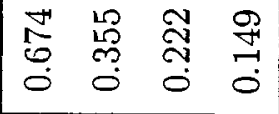 & 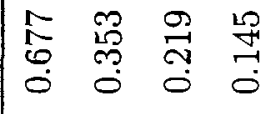 & 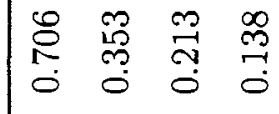 & 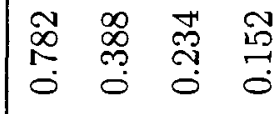 \\
\hline & 点 & 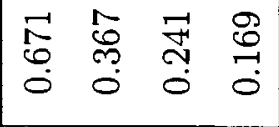 & 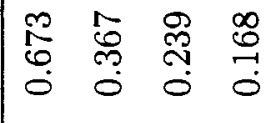 & 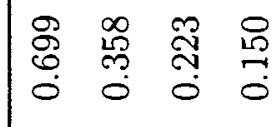 & $\mid \begin{array}{cccc}\infty & -1 & 0 & 0 \\
0 & F & 0 \\
0 & 0 & 0 & 0 \\
0 & 0 & 0\end{array}$ \\
\hline & $\begin{array}{l}\text { is } \\
\text { ĩ }\end{array}$ & 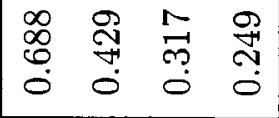 & 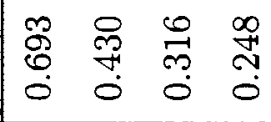 & 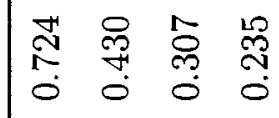 & 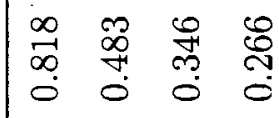 \\
\hline & 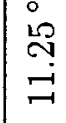 & 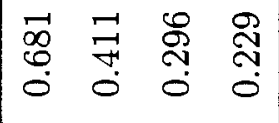 & 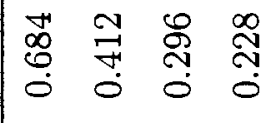 & 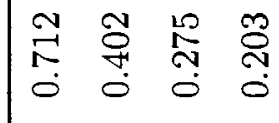 & 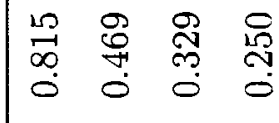 \\
\hline & $\stackrel{\circ}{\circ}$ & 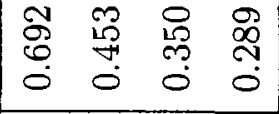 & 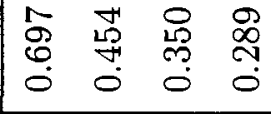 & 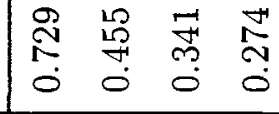 & 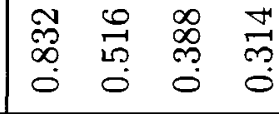 \\
\hline & & 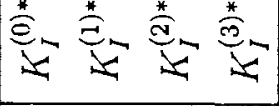 & 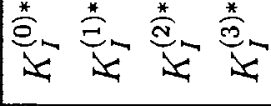 & 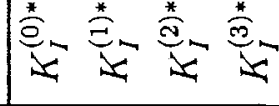 & 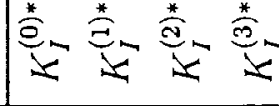 \\
\hline & $\frac{5}{5}$ & $\stackrel{\Upsilon}{\varrho}$ & $\stackrel{\leftrightarrow}{C}$ & $\ddot{0}$ & $\stackrel{\infty}{0}$ \\
\hline
\end{tabular}




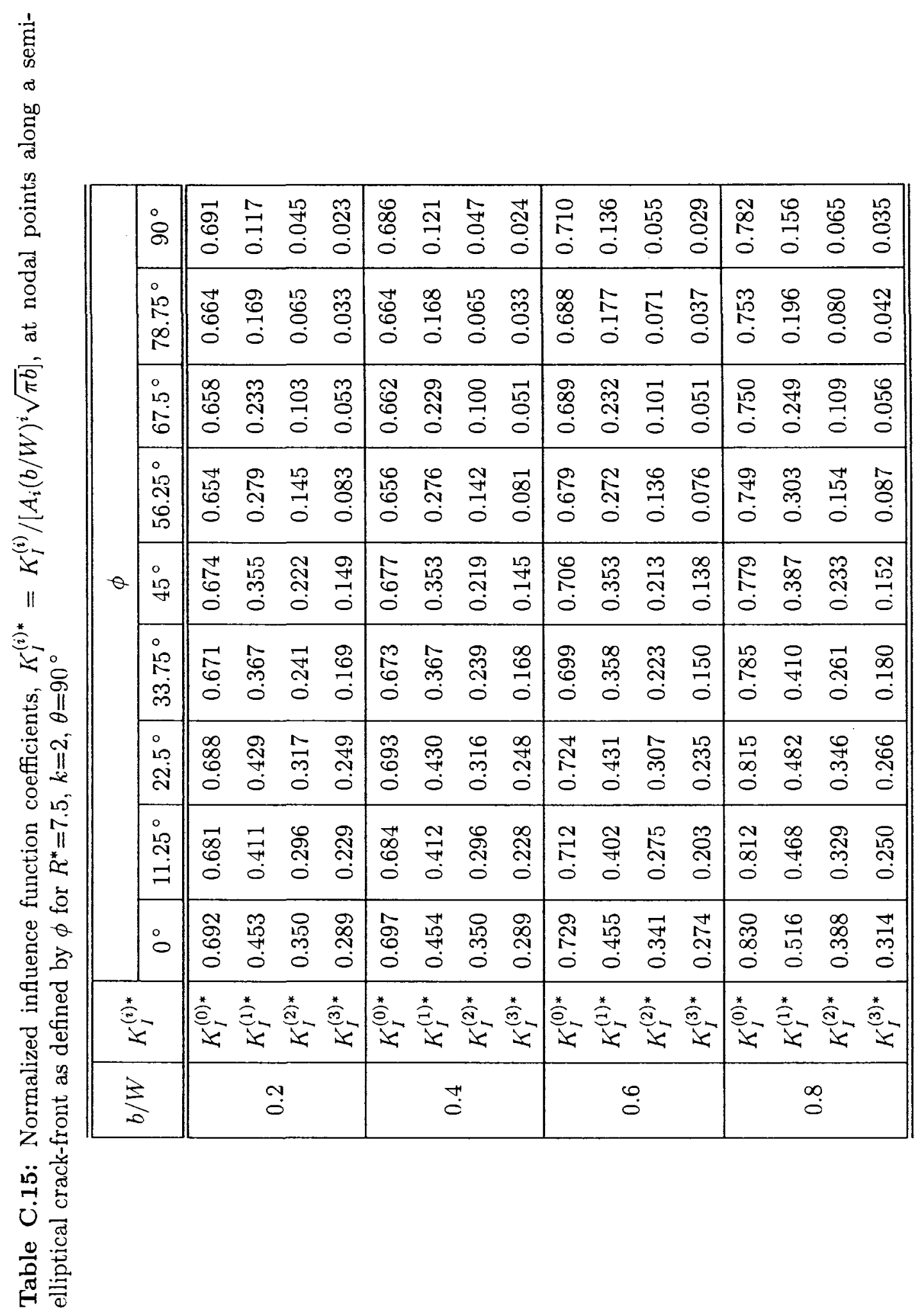




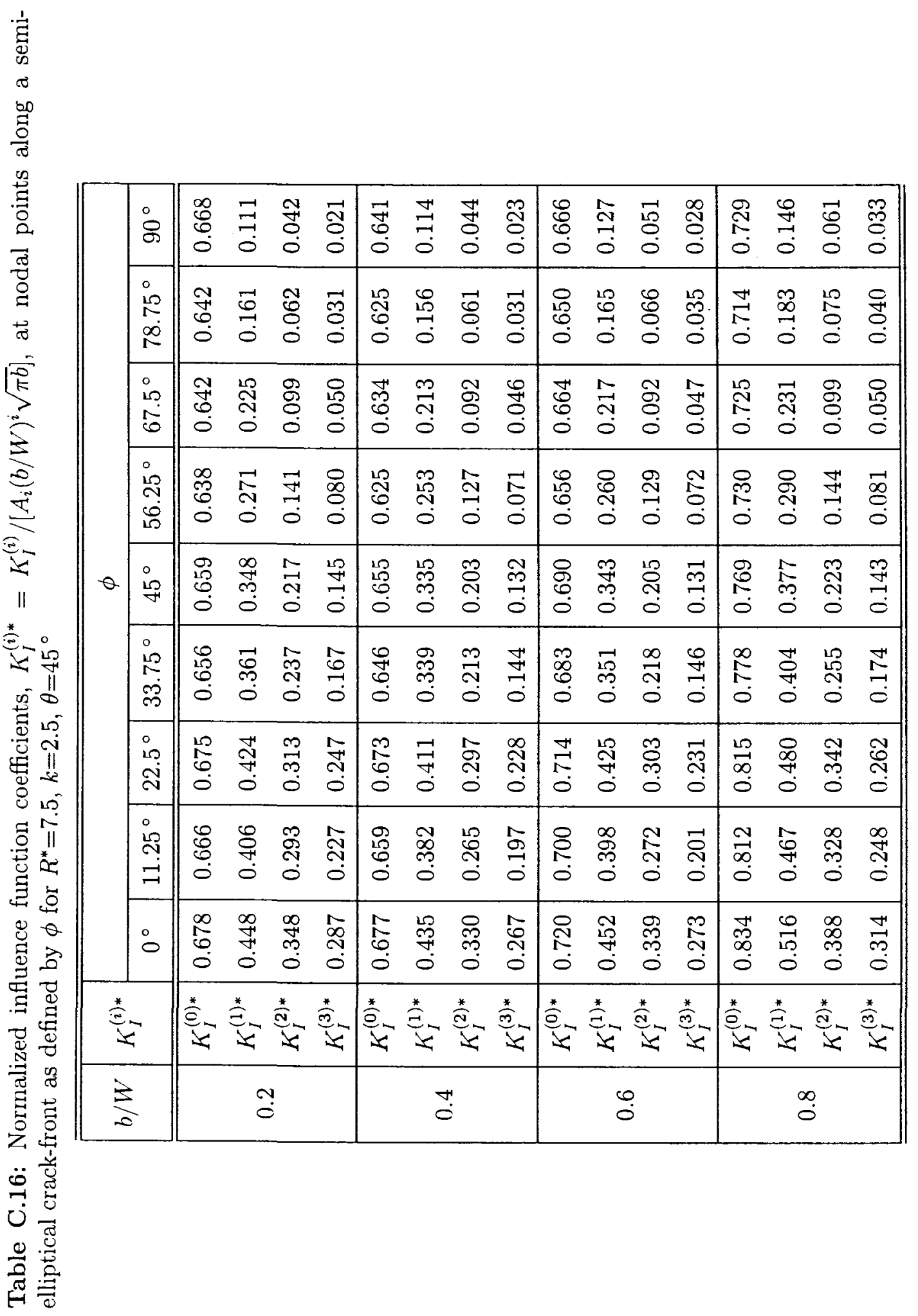




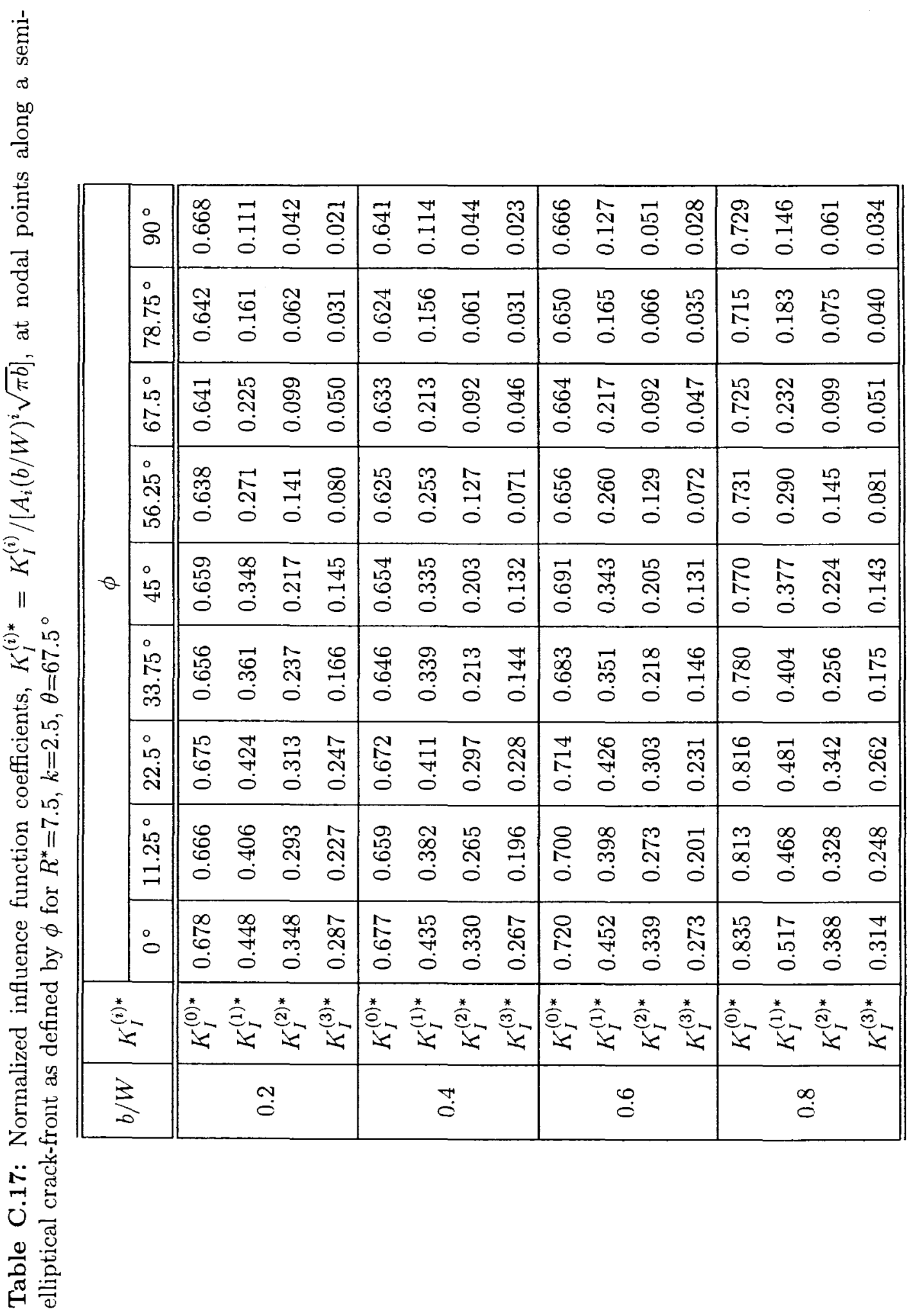




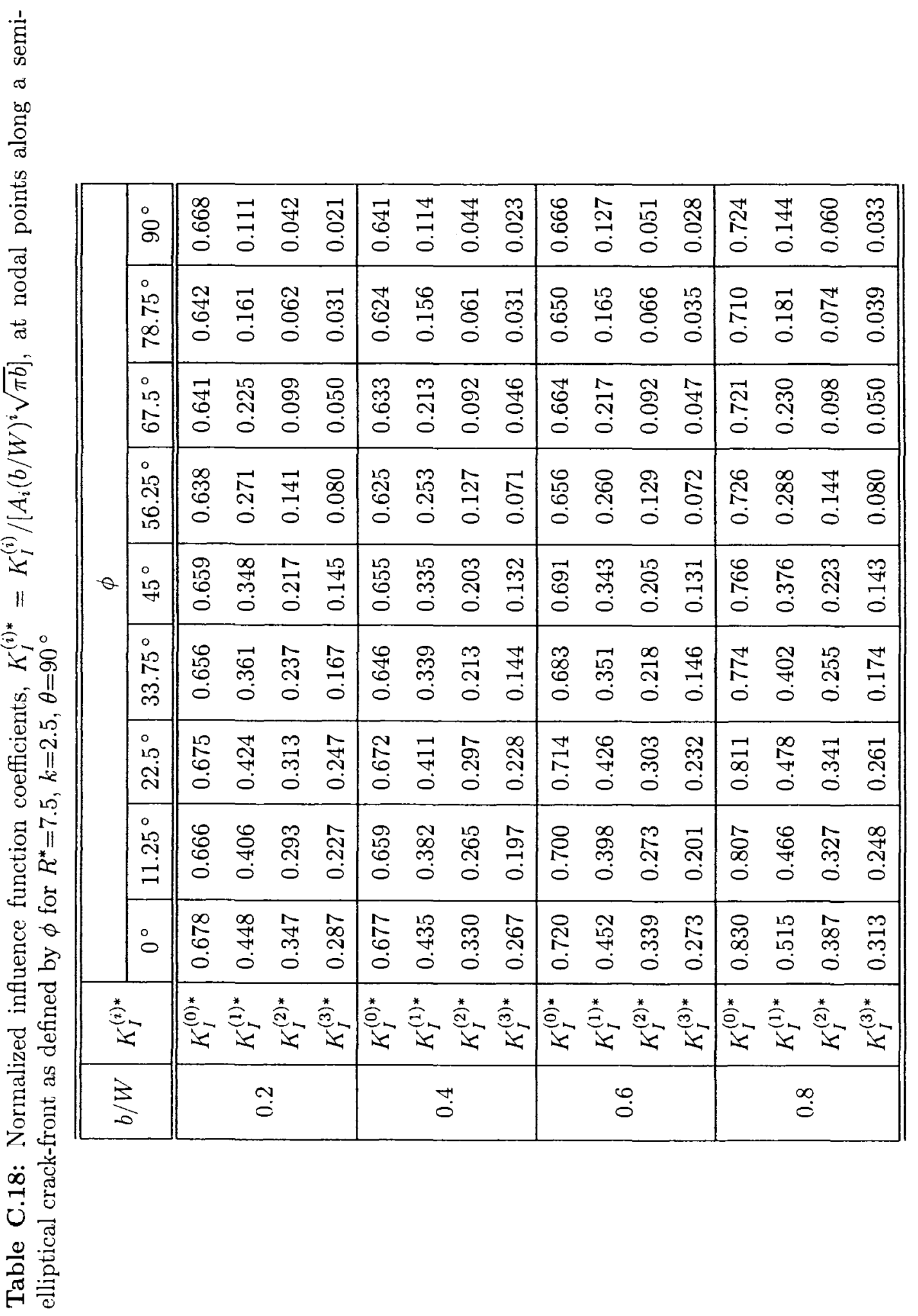




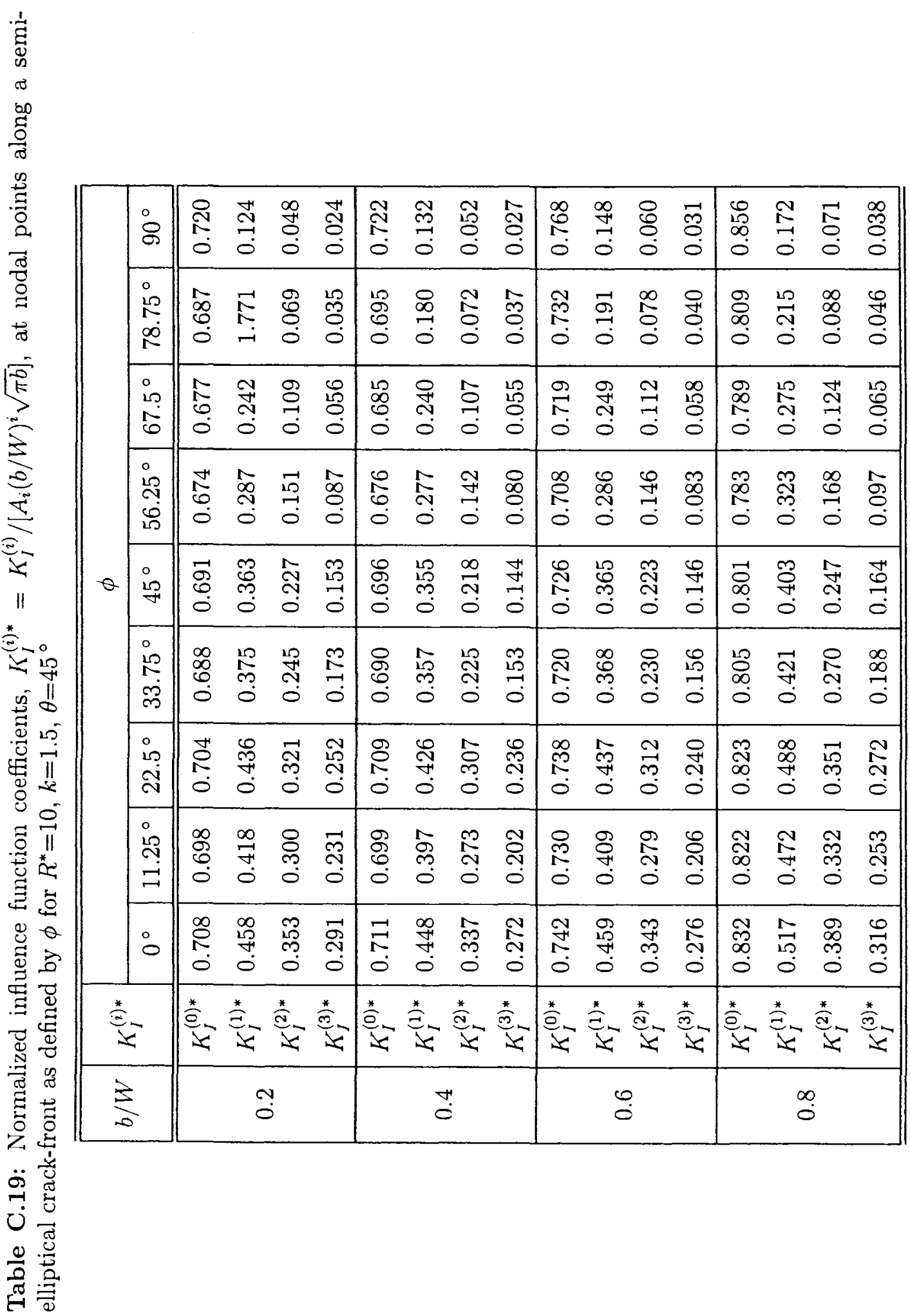




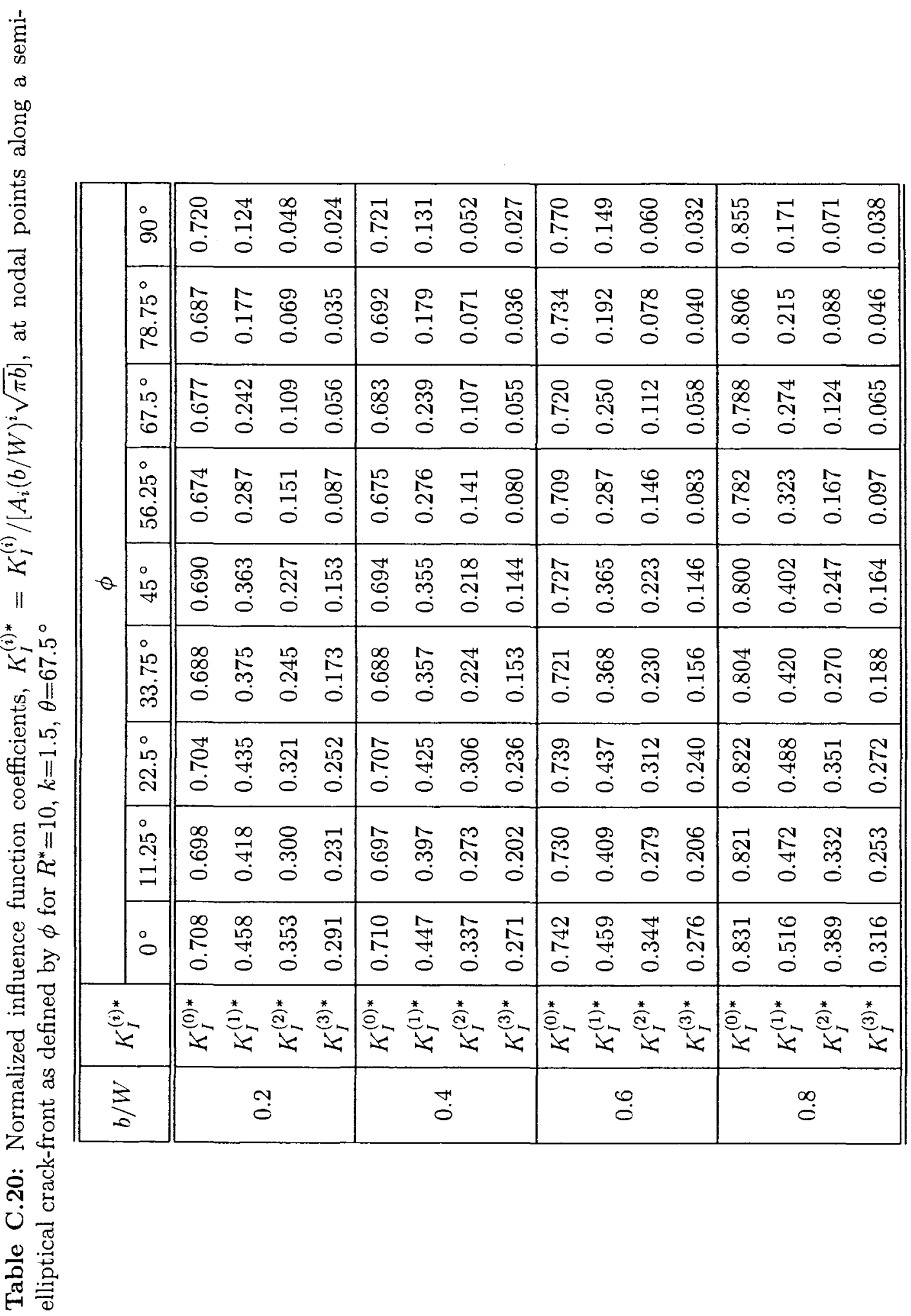




\begin{tabular}{|c|c|c|c|c|c|}
\hline \multirow{9}{*}{$\theta$} & 8 & 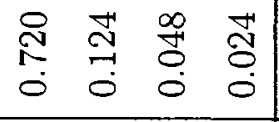 & 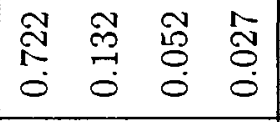 & 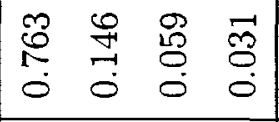 & $\mid \begin{array}{cccc}N & 0 & 0 & 0 \\
0 & 1 & 0 & 0 \\
0 & 0 & 0 \\
0 & 0 & 0 & 0 \\
0\end{array}$ \\
\hline & 帒 & 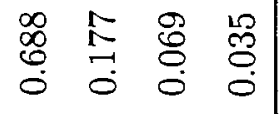 & 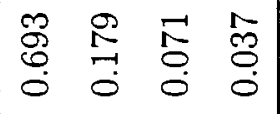 & 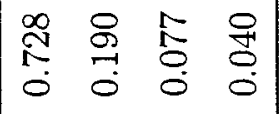 & 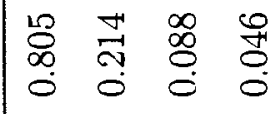 \\
\hline & 范 & 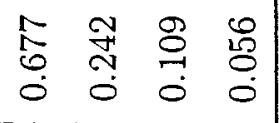 & $\mid \begin{array}{llll}0 & 0 & 1 & 1 \\
0 & \stackrel{f}{0} & 0 & 0 \\
0 & 0 & 0 & 0 \\
0 & 0 & 0 & 0 \\
0\end{array}$ & 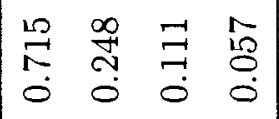 & 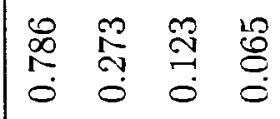 \\
\hline & $\begin{array}{l}\text { i⿱ } \\
\text { ஸे } \\
\text { in }\end{array}$ & 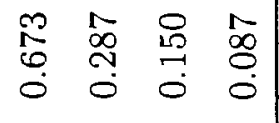 & 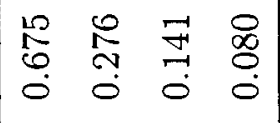 & 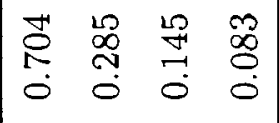 & 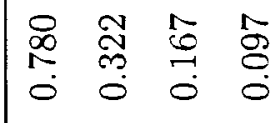 \\
\hline & i⿱ 口i & 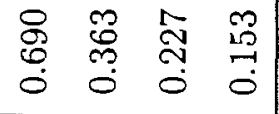 & 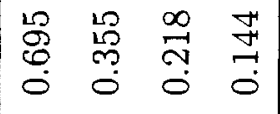 & 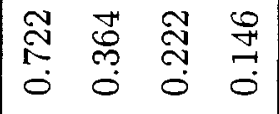 & 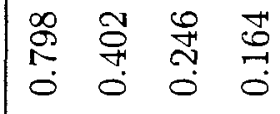 \\
\hline & 点 & 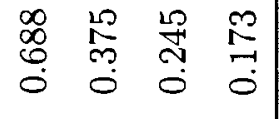 & 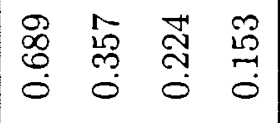 & 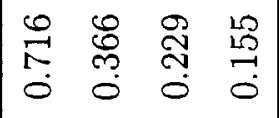 & $\mid \begin{array}{cccc}\overrightarrow{1} & 0 & 0 & \infty \\
\infty & F & 0 & \infty \\
0 & 0 & 0 & 0 \\
0 & 0\end{array}$ \\
\hline & 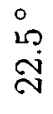 & 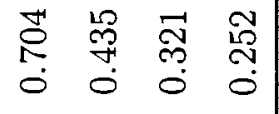 & 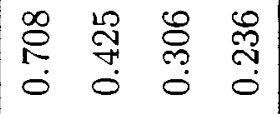 & 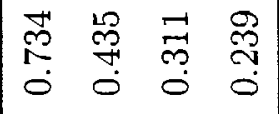 & 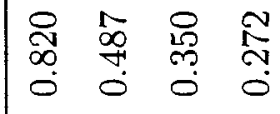 \\
\hline & 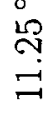 & 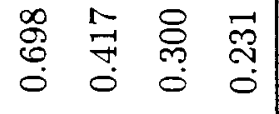 & 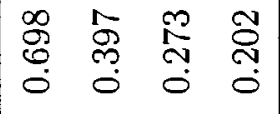 & 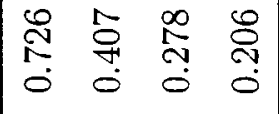 & 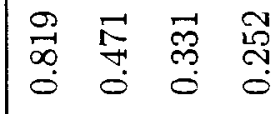 \\
\hline & $\stackrel{0}{\circ}$ & 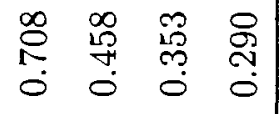 & 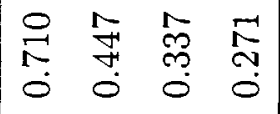 & 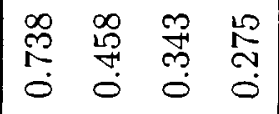 & $\mid \begin{array}{cccc}9 & 0 & 0 & 20 \\
0 & 0 & 0 & 0 \\
0 & 10 & 0 \\
0 & 0 & 0 & 0\end{array}$ \\
\hline & & 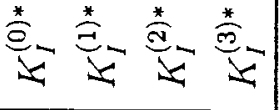 & 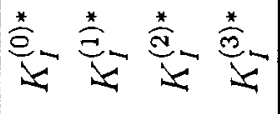 & 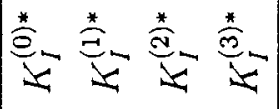 & 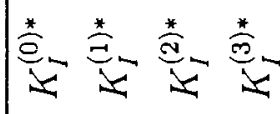 \\
\hline & & ๙ֶ. & $\stackrel{\vec{c}}{c}$ & $\stackrel{0}{0}$ & $\stackrel{\infty}{0}$ \\
\hline
\end{tabular}




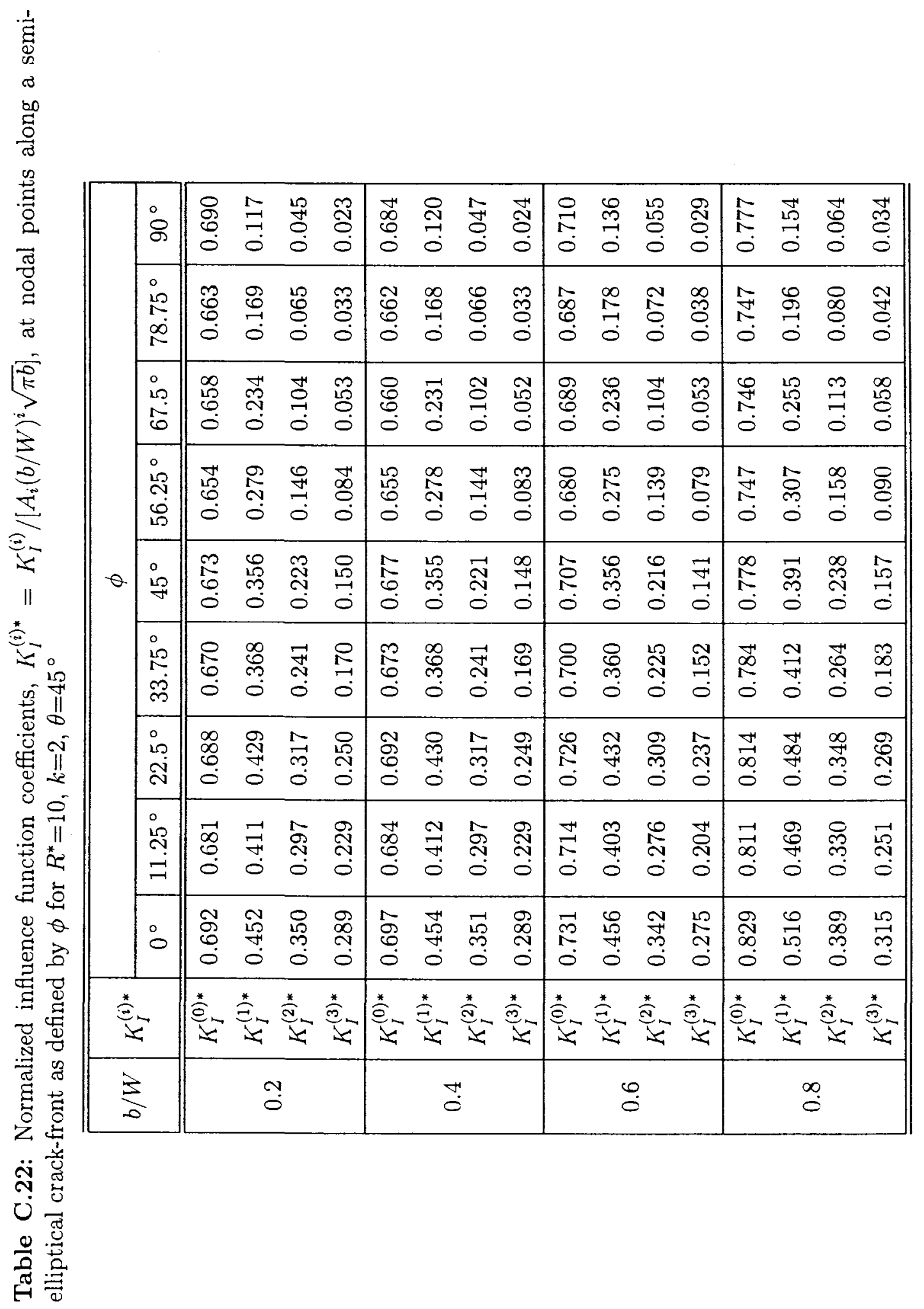




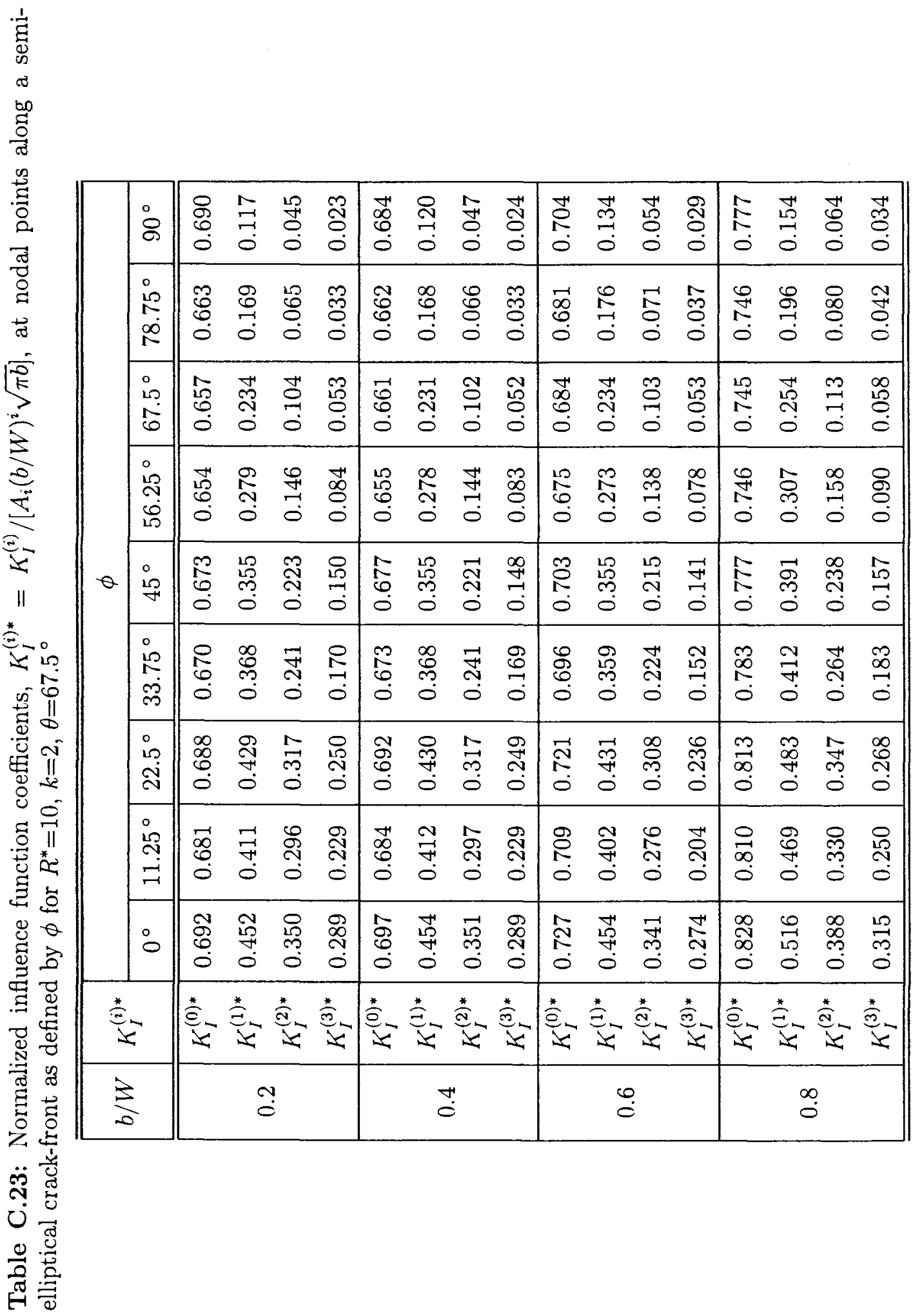


$\stackrel{*}{\stackrel{*}{\ominus}}$

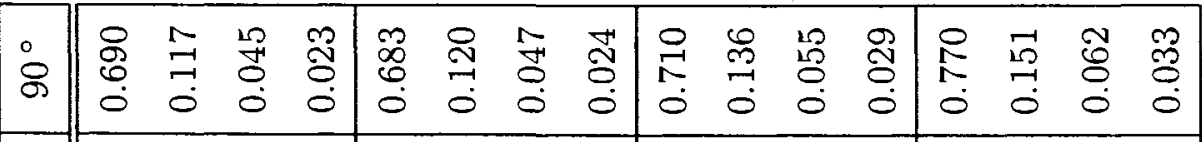

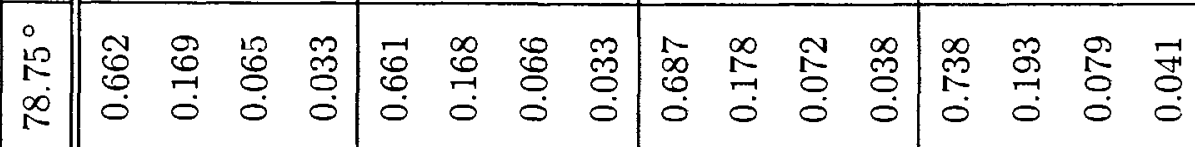

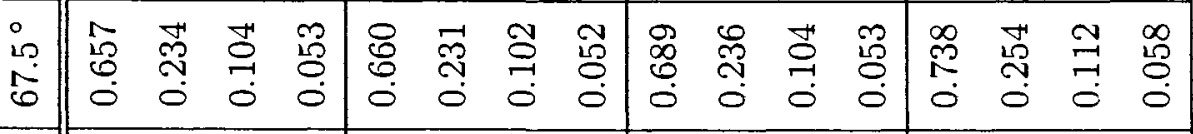

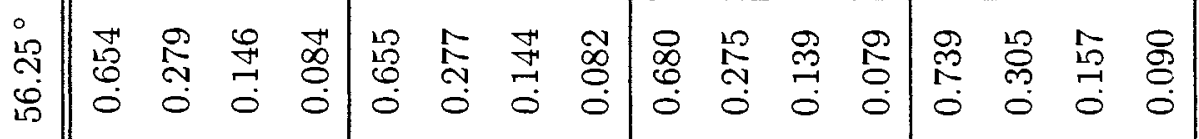

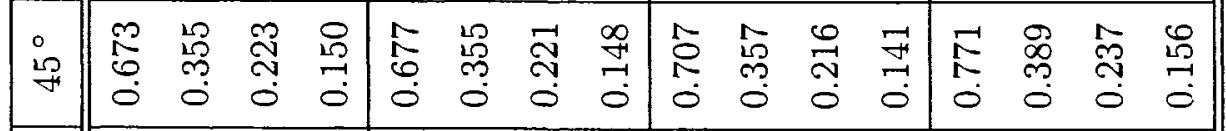
안 is 8 䒼 芦 离 ت잉

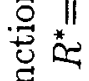
壳它 $\ddot{\theta}$

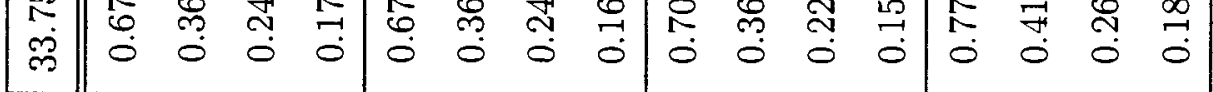

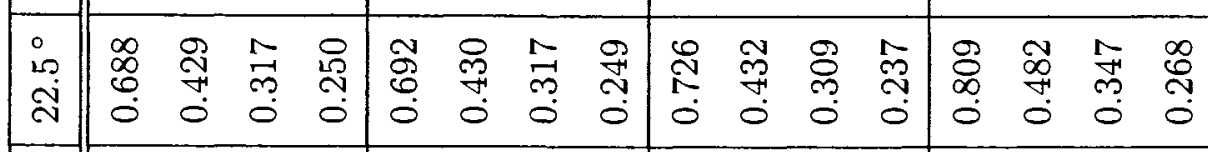

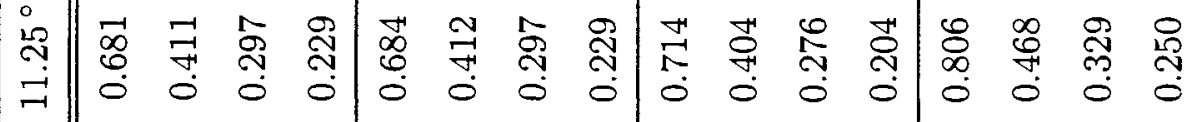

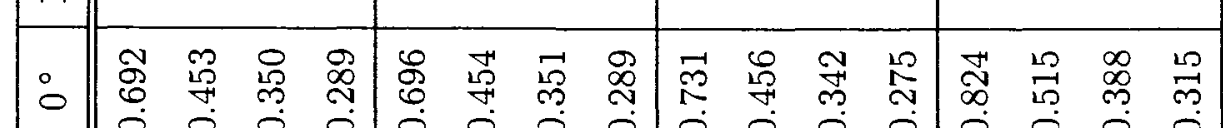
疍 .

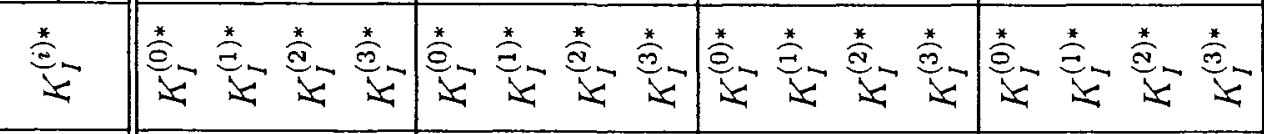
\begin{tabular}{|l|l|l|l}
\hline \multirow{2}{*}{} & $\stackrel{\leftrightarrow}{0}$ & 0 & 0 \\
\hline
\end{tabular} 


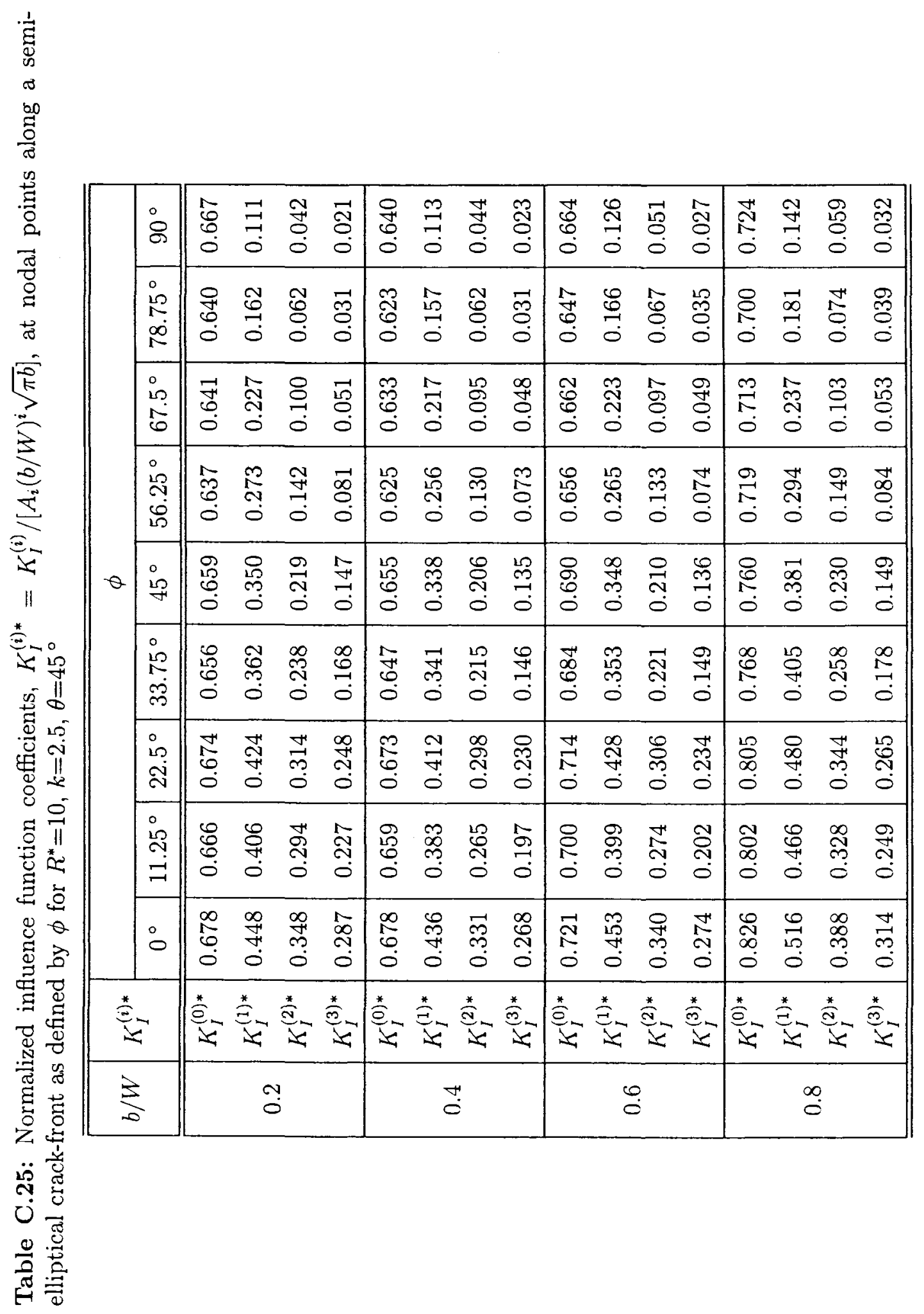




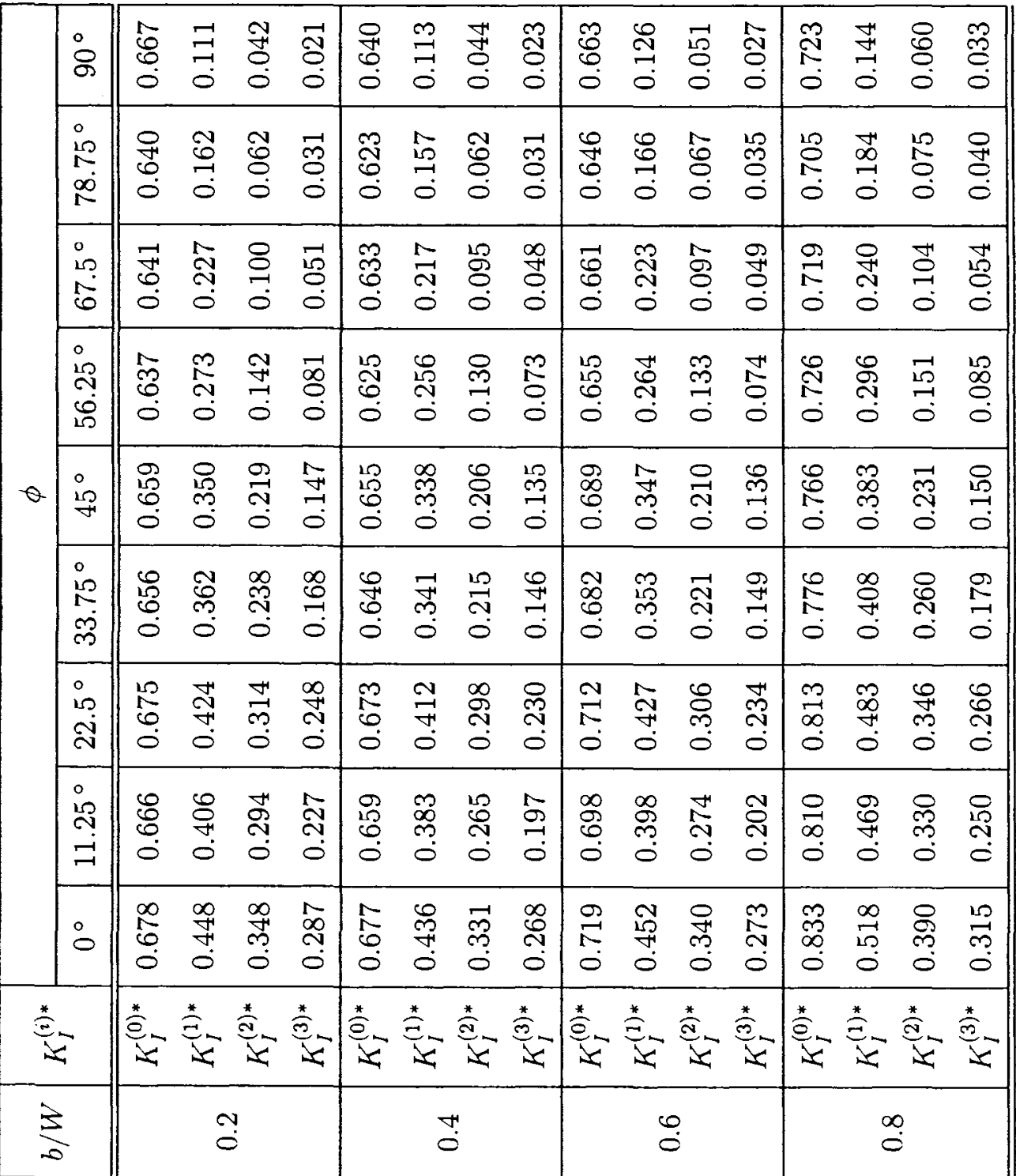

乙

$\ddot{0}$ 


\begin{tabular}{|c|c|c|c|c|c|c|c|c|c|c|c|c|c|c|c|c|c|}
\hline \multirow{9}{*}{$a$} & 8 & $\begin{array}{l}1 \\
\stackrel{8}{0} \\
0\end{array}$ & $\underset{\vec{\sigma}}{\vec{\Xi}}$ & $\begin{array}{l}\stackrel{\sim}{J} \\
\stackrel{0}{0}\end{array}$ & $\begin{array}{l}\overrightarrow{\widehat{O}} \\
\stackrel{\circ}{0}\end{array}$ & $\begin{array}{l}\text { 유 } \\
\text { O } \\
\stackrel{0}{0}\end{array}$ & $\stackrel{m}{\vec{\partial}}$ & $\begin{array}{l}\stackrel{H}{H} \\
\stackrel{0}{0}\end{array}$ & 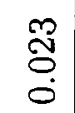 & $\begin{array}{l}\mathscr{3} \\
\stackrel{0}{0} \\
0\end{array}$ & $\underset{\stackrel{0}{\overparen{I}}}{\stackrel{0}{0}}$ & 봉 & $\stackrel{ }{O}$ & $\vec{尺}$ & $\stackrel{\mathscr{O}}{\stackrel{\leftrightarrow}{:}}$ & $\stackrel{\overrightarrow{8}}{\stackrel{8}{0}}$ & $\stackrel{\substack{0 \\
0}}{0}$ \\
\hline & $\begin{array}{l}10 \\
10 \\
0 \\
1\end{array}$ & 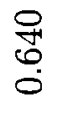 & $\begin{array}{l}\underset{\mathscr{O}}{0} \\
\stackrel{-}{0}\end{array}$ & 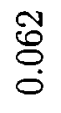 & $\ddot{\ddot{\theta}}$ & 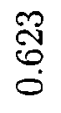 & $\begin{array}{l}\stackrel{10}{10} \\
\stackrel{0}{0}\end{array}$ & $\begin{array}{l}\widetilde{O} \\
\stackrel{8}{0}\end{array}$ & $\stackrel{\vec{~}}{0}$ & $\begin{array}{l}\stackrel{0}{\sharp} \\
\stackrel{\leftrightarrow}{0} \\
0\end{array}$ & $\begin{array}{l}\mathscr{0} \\
\stackrel{0}{0}\end{array}$ & 占 & 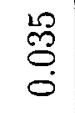 & $\stackrel{g}{P}$ & $\begin{array}{l}\stackrel{\infty}{\infty} \\
\stackrel{0}{0}\end{array}$ & $\begin{array}{l}0 \\
\stackrel{0}{0} \\
0\end{array}$ & 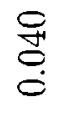 \\
\hline & مَ & ت্ّ & 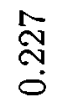 & $\begin{array}{l}8 \\
\stackrel{8}{0}\end{array}$ & $\begin{array}{l}\overrightarrow{10} \\
\stackrel{0}{0}\end{array}$ & $\begin{array}{l}\mathscr{\mathscr { T }} \\
\stackrel{0}{0} \\
\stackrel{0}{0}\end{array}$ & $\underset{\sim}{\sim}$ & $\begin{array}{l}10 \\
\stackrel{8}{\circ} \\
\circ\end{array}$ & $\begin{array}{l}\stackrel{\infty}{\ddagger} \\
\stackrel{Ð}{0}\end{array}$ & $\begin{array}{l}\overrightarrow{0} \\
\ddot{0}\end{array}$ & ָָ & $\stackrel{ }{\circ}$ & $\stackrel{g}{\stackrel{g}{0}}$ & $\stackrel{\vec{N}}{0}$ & 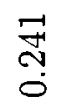 & $\begin{array}{l}\stackrel{2}{9} \\
\stackrel{1}{0} \\
0\end{array}$ & $\stackrel{\stackrel{H}{0}}{\stackrel{H}{0}}$ \\
\hline & 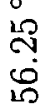 & $\begin{array}{l}\hat{M} \\
\stackrel{0}{0} \\
\dot{0}\end{array}$ & 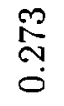 & $\underset{\stackrel{F}{\rightleftarrows}}{\stackrel{\sim}{\leftrightarrows}}$ & $\begin{array}{l}\vec{\infty} \\
\stackrel{0}{0} \\
0\end{array}$ & 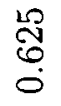 & 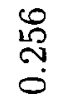 & $\stackrel{\stackrel{9}{9}}{\stackrel{0}{0}}$ & 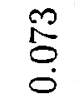 & $\begin{array}{l}\stackrel{4}{0} \\
\stackrel{8}{0} \\
\stackrel{0}{0}\end{array}$ & 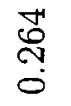 & 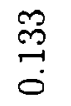 & $\begin{array}{l}\text { N } \\
\text { Oे } \\
0\end{array}$ & 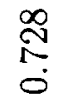 & 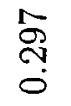 & $\begin{array}{l}\overrightarrow{50} \\
\stackrel{2}{0}\end{array}$ & $\stackrel{\mathscr{\infty}}{\circ}$ \\
\hline & is & $\begin{array}{l}\text { in } \\
0 \\
0 \\
0\end{array}$ & $\begin{array}{l}\text { 只 } \\
\text { m } \\
0\end{array}$ & $\begin{array}{l}\stackrel{\sigma}{\sim} \\
\stackrel{\sim}{0}\end{array}$ & 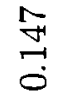 & $\begin{array}{l}10 \\
18 \\
0 \\
0 \\
0\end{array}$ & 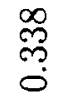 & 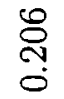 & $\stackrel{\stackrel{L 2}{\dddot{m}}}{\stackrel{0}{0}}$ & $\begin{array}{l}0 \\
\infty \\
0 \\
0\end{array}$ & 今ે & 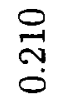 & $\begin{array}{l}0 \\
\stackrel{2}{!} \\
0\end{array}$ & $\begin{array}{l}\stackrel{8}{0} \\
0 \\
0\end{array}$ & $\begin{array}{l}\stackrel{\infty}{\infty} \\
\text { r } \\
0\end{array}$ & ָิ & $\stackrel{50}{0}$ \\
\hline & $\begin{array}{l}\stackrel{0}{N} \\
\stackrel{n}{n} \\
\text { m }\end{array}$ & $\begin{array}{l}0 \\
18 \\
\stackrel{0}{0} \\
0\end{array}$ & $\begin{array}{l}\text { @્ } \\
\text { లె } \\
0\end{array}$ & \begin{tabular}{l}
$\infty$ \\
$\stackrel{2}{\sim}$ \\
\hdashline
\end{tabular} & $\begin{array}{l}\infty \\
\stackrel{0}{0} \\
0\end{array}$ & 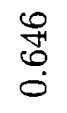 & 芦 & 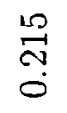 & 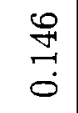 & $\begin{array}{l}\text { Oै } \\
0 \\
0 \\
0\end{array}$ & 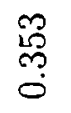 & 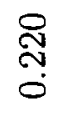 & 㞼 & $\begin{array}{l}\infty \\
\stackrel{1}{1} \\
0\end{array}$ & 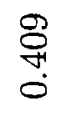 & $\begin{array}{l}\overrightarrow{0} \\
\text { ஸे }\end{array}$ & $\stackrel{\mathscr{\infty}}{\stackrel{0}{0}}$ \\
\hline & $\begin{array}{l}\text { in } \\
\text { ๙ิ }\end{array}$ & $\begin{array}{l}\stackrel{N}{0} \\
0 \\
0\end{array}$ & $\underset{\stackrel{\leftrightarrow}{*}}{\stackrel{H}{0}}$ & $\underset{ٌ}{\stackrel{\sharp}{0}}$ & $\underset{\stackrel{\infty}{N}}{\stackrel{\infty}{\sim}}$ & $\begin{array}{l}\stackrel{?}{0} \\
0 \\
0\end{array}$ & 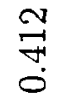 & $\begin{array}{l}\infty \\
\stackrel{\sim}{్} \\
0\end{array}$ & 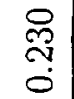 & $\stackrel{\sim}{\stackrel{F}{\leftrightarrows}}$ & 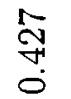 & $\begin{array}{l}0 \\
\text { ల్ } \\
0\end{array}$ & 心ָ & $\begin{array}{l}10 \\
\infty \\
0 \\
0\end{array}$ & $\begin{array}{l}\stackrel{+}{\infty} \\
\stackrel{0}{0}\end{array}$ & 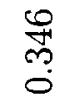 & 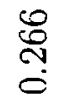 \\
\hline & $\stackrel{\stackrel{\sim}{\sim}}{\stackrel{\sim}{\rightleftarrows}}$ & $\begin{array}{l}\mathscr{0} \\
\stackrel{8}{0} \\
0\end{array}$ & $\begin{array}{l}\stackrel{8}{8} \\
\stackrel{1}{0}\end{array}$ & ণ্ঠু & 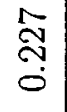 & $\begin{array}{l}\stackrel{9}{8} \\
\stackrel{8}{0} \\
0 \\
0\end{array}$ & $\begin{array}{l}\mathscr{\infty} \\
\stackrel{\infty}{0} \\
0\end{array}$ & $\begin{array}{l}\stackrel{2}{0} \\
\stackrel{0}{0} \\
0\end{array}$ & $\stackrel{\stackrel{ }{g}}{\stackrel{0}{0}}$ & $\begin{array}{l}\infty \\
\stackrel{\infty}{0} \\
0\end{array}$ & 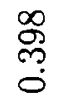 & 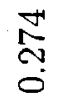 & $\begin{array}{l}\text { ్ֶ } \\
\text { O̦ }\end{array}$ & 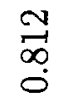 & $\stackrel{R}{\stackrel{R}{+}}$ & 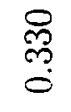 & 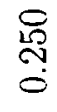 \\
\hline & : & & 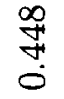 & 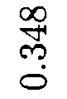 & \begin{tabular}{l}
$\stackrel{N}{\infty}$ \\
\multirow{0}{0}{} \\
0
\end{tabular} & $\begin{array}{l}\hat{N} \\
\hat{0} \\
0\end{array}$ & $\begin{array}{l}\stackrel{\mathscr{7}}{\Im} \\
0\end{array}$ & $\stackrel{\vec{m}}{\stackrel{0}{0}}$ & $\begin{array}{l}\infty \\
\stackrel{\infty}{0} \\
0\end{array}$ & & 疍 & 융 & 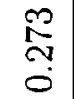 & $\begin{array}{l}\stackrel{L}{\varpi} \\
\stackrel{\infty}{0} \\
0\end{array}$ & $\underset{0}{\stackrel{0}{b 3}}$ & ஓ్ల & $\stackrel{\oplus}{\stackrel{m}{\varrho}}$ \\
\hline & & 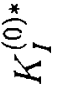 & \pm & 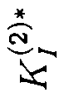 & $\stackrel{*}{\stackrel{*}{\rho}}$ & $\stackrel{*}{\stackrel{*}{e}}$ & & 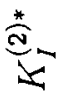 & 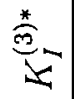 & & & 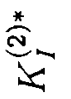 & $\underbrace{*}$ & & & & $\stackrel{*}{\stackrel{*}{0}}$ \\
\hline & & & & & & & 0 & & & & & & & & & & \\
\hline
\end{tabular}

芥芯 
C.2 Stress Intensity Factors from Influence Coefficients 


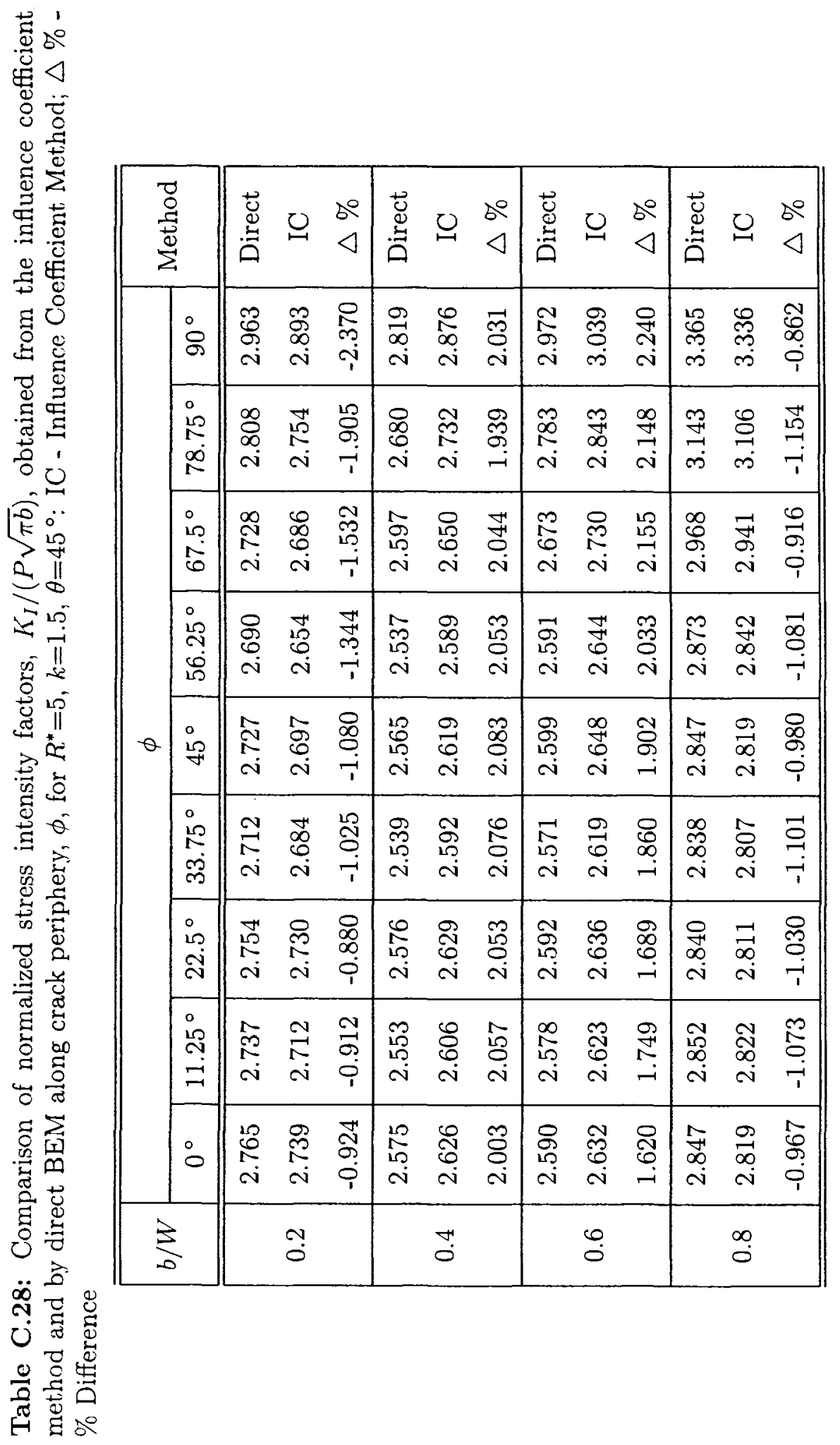




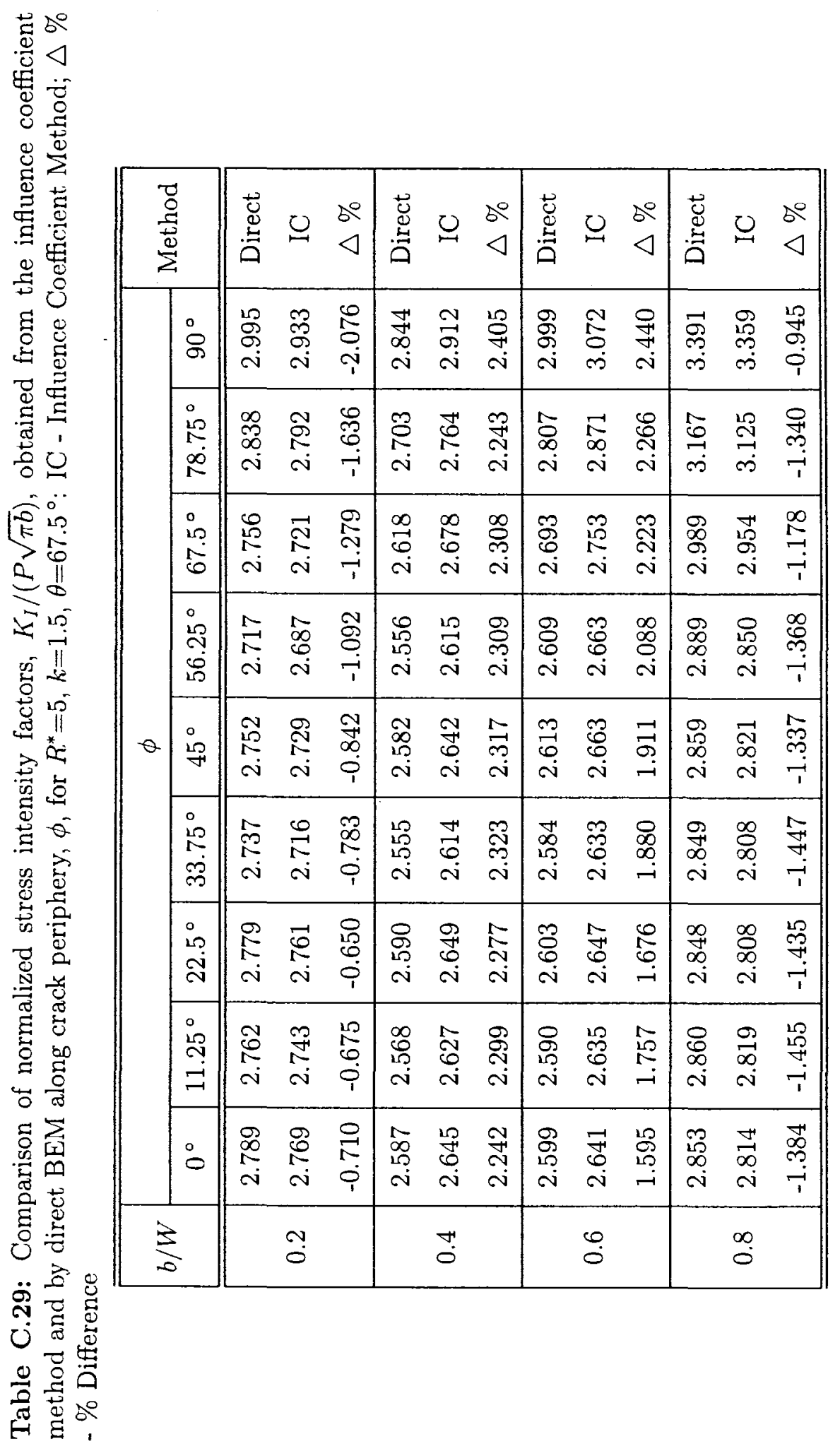




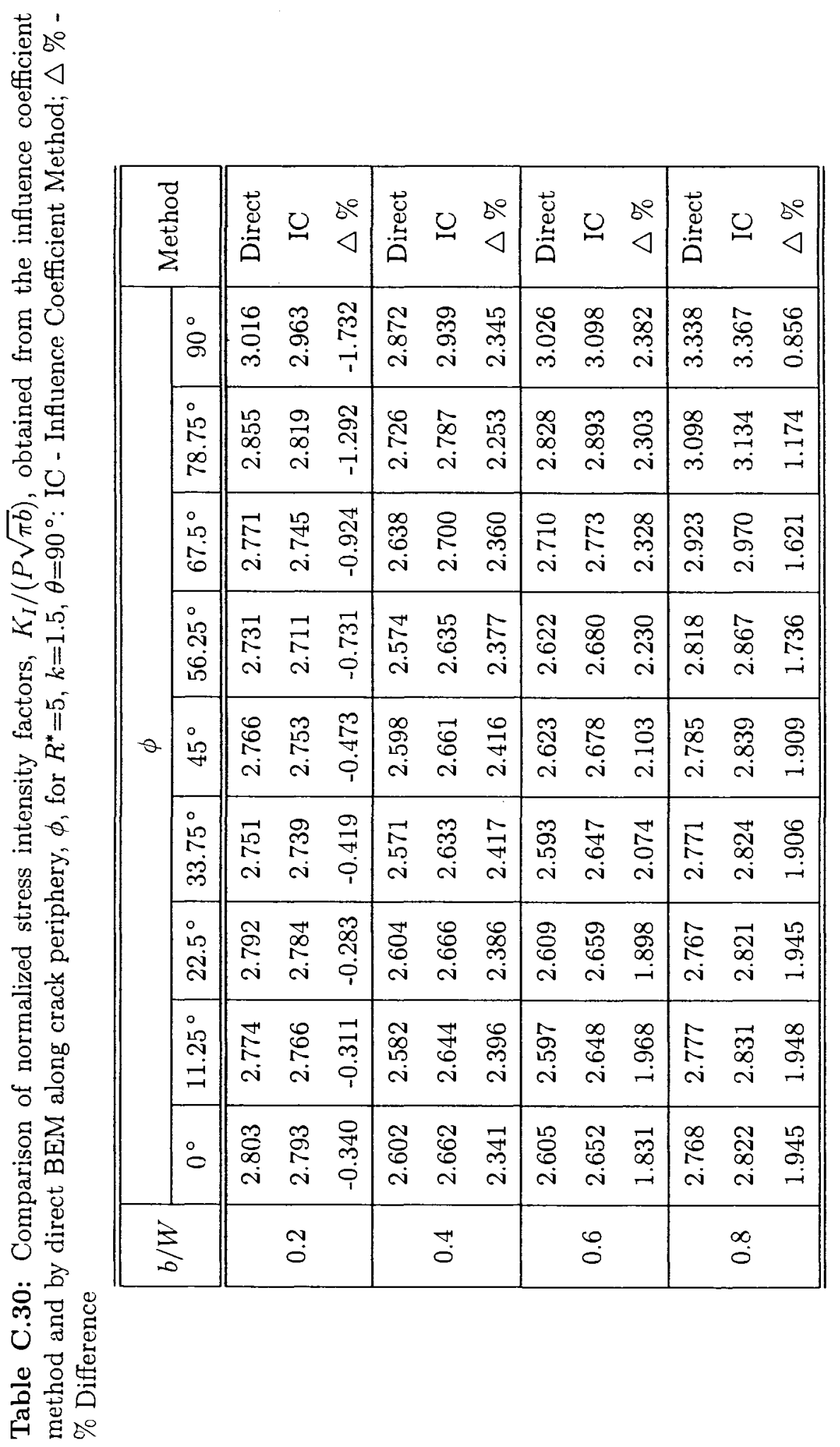




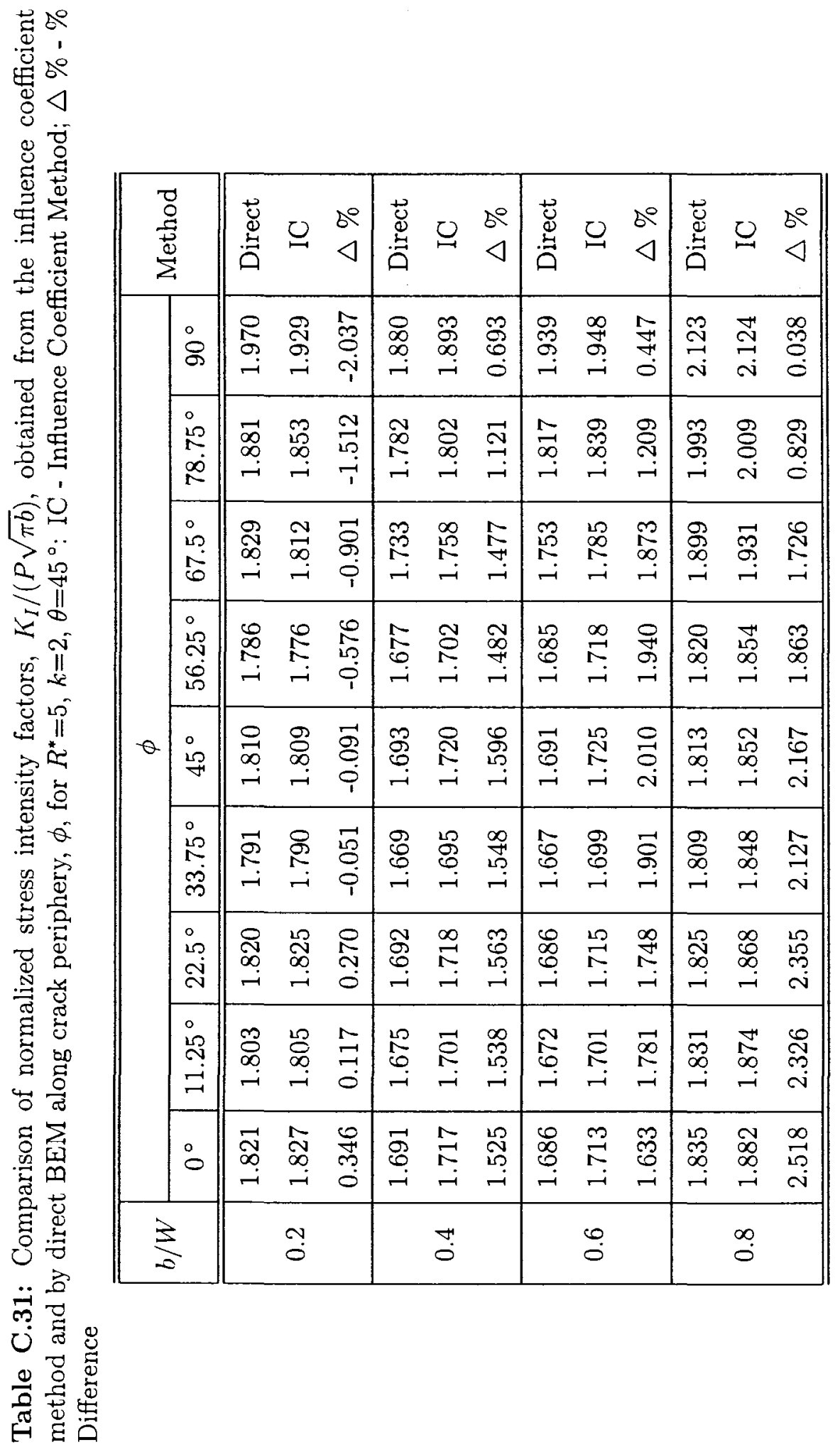




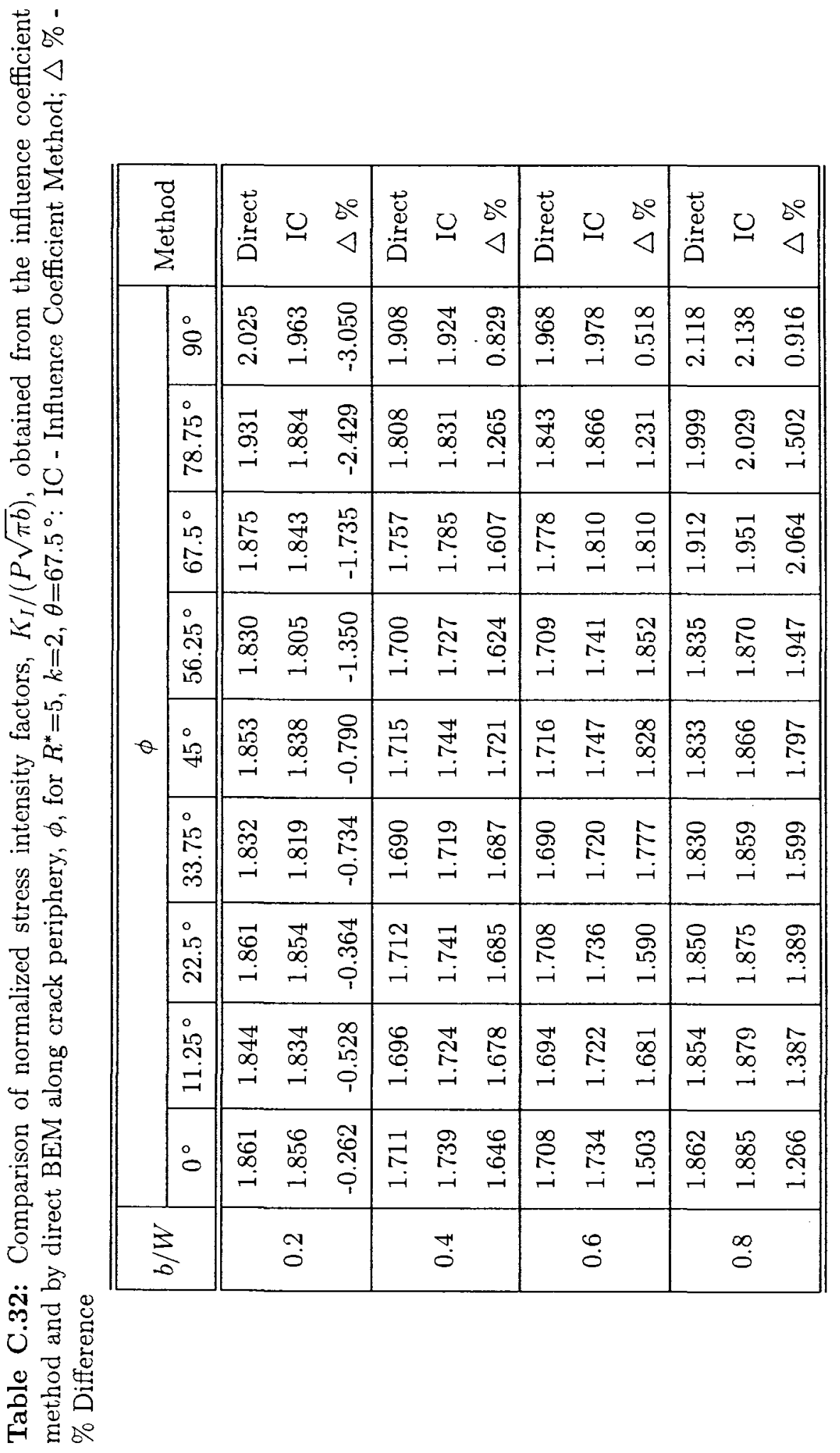




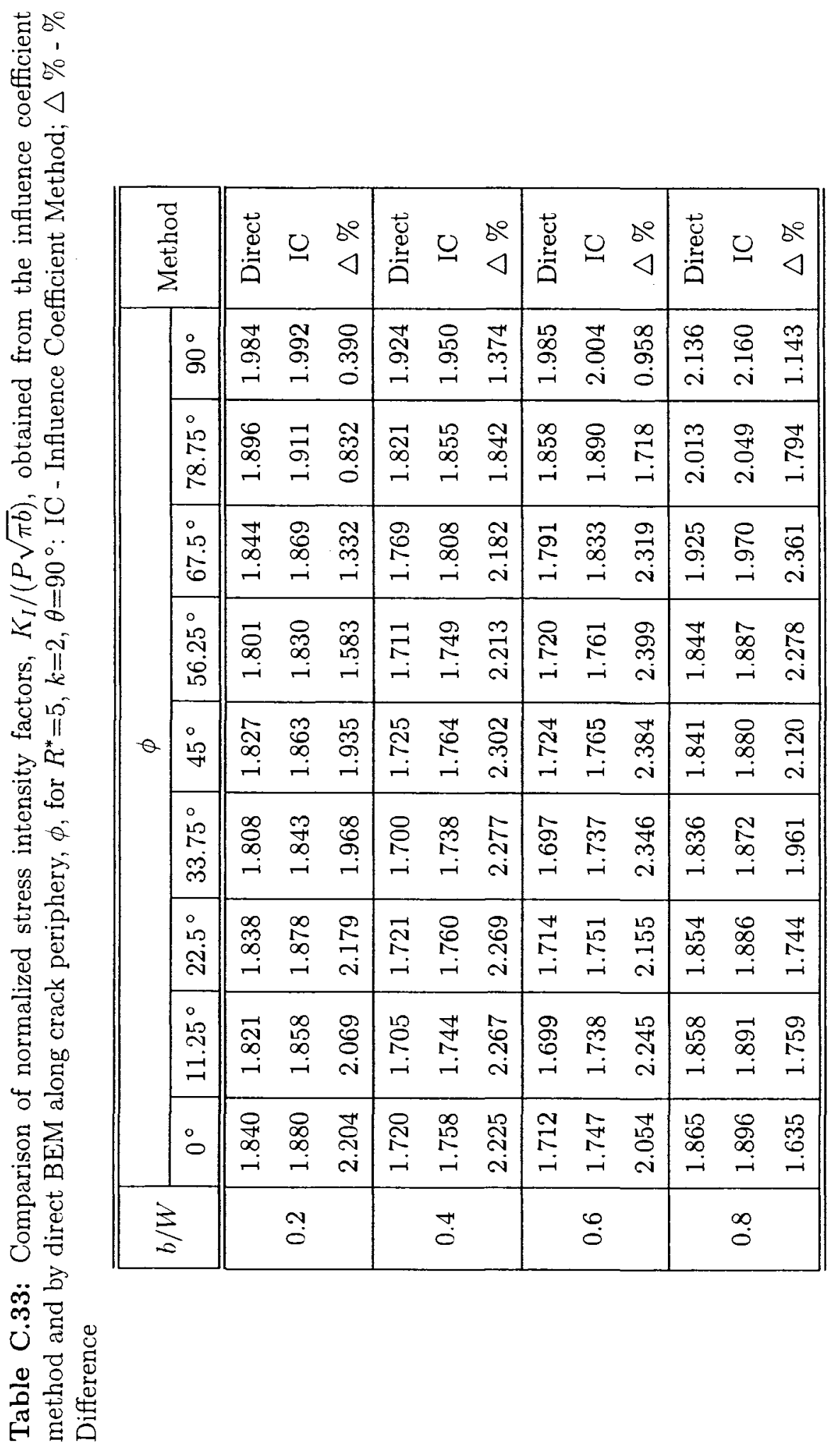




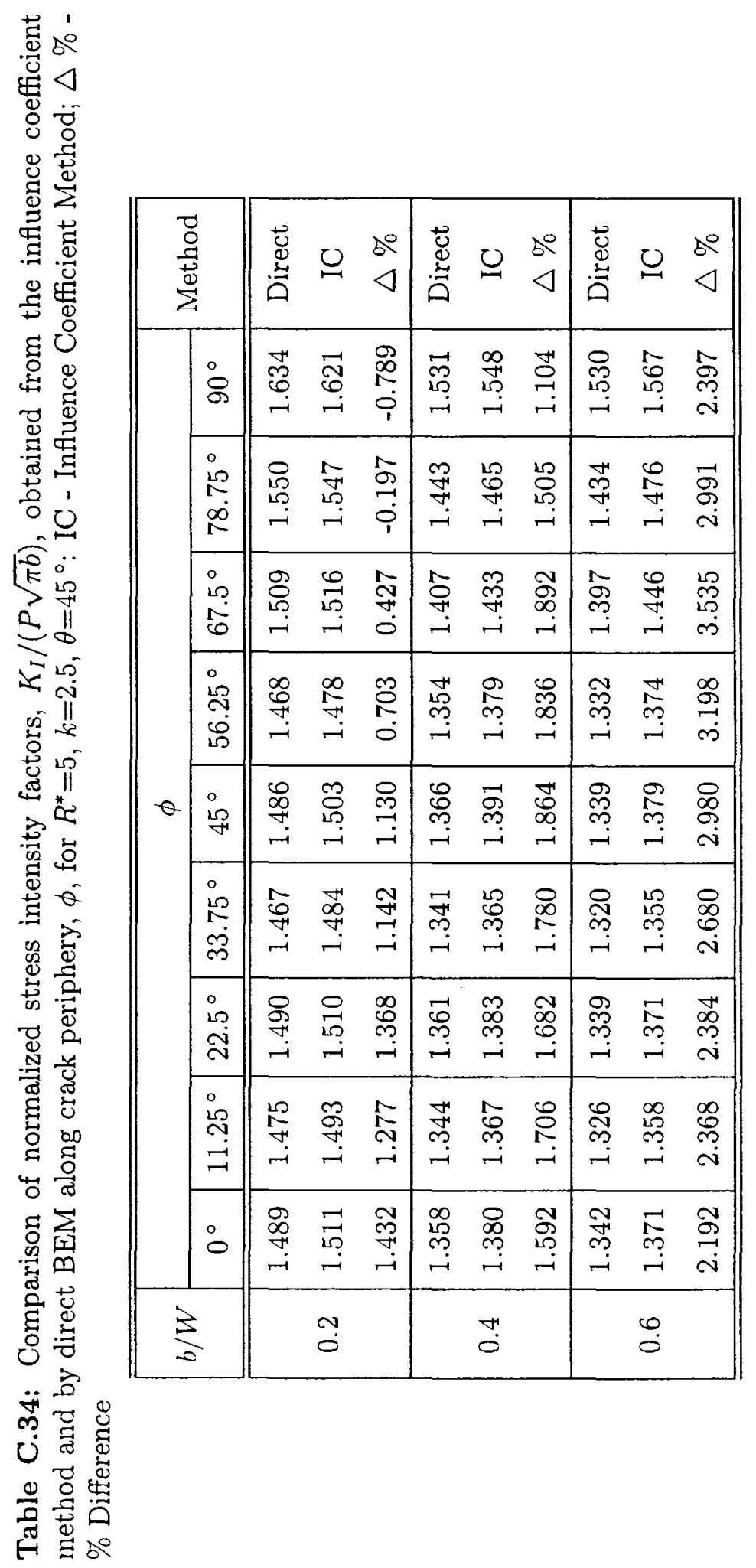




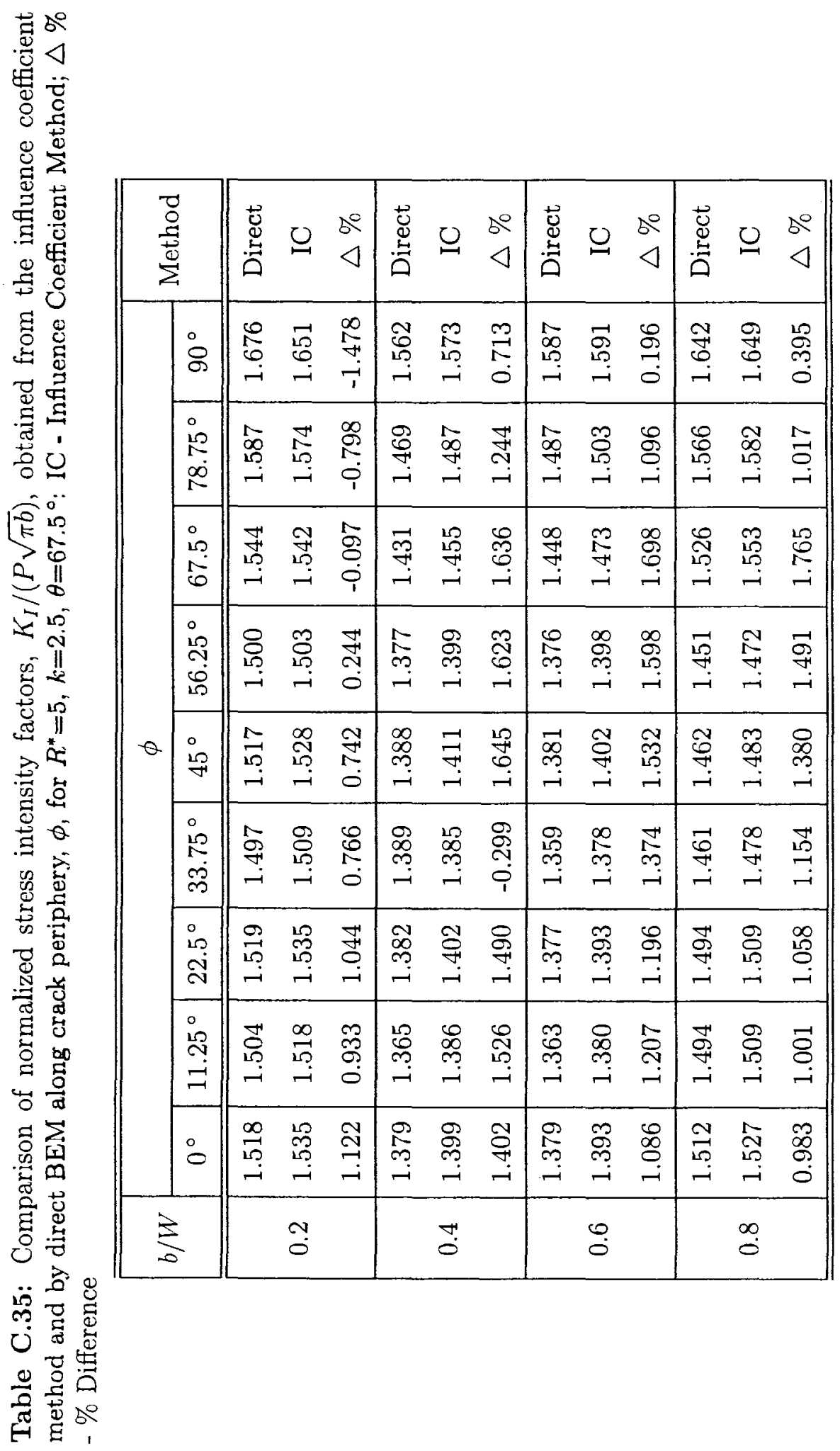




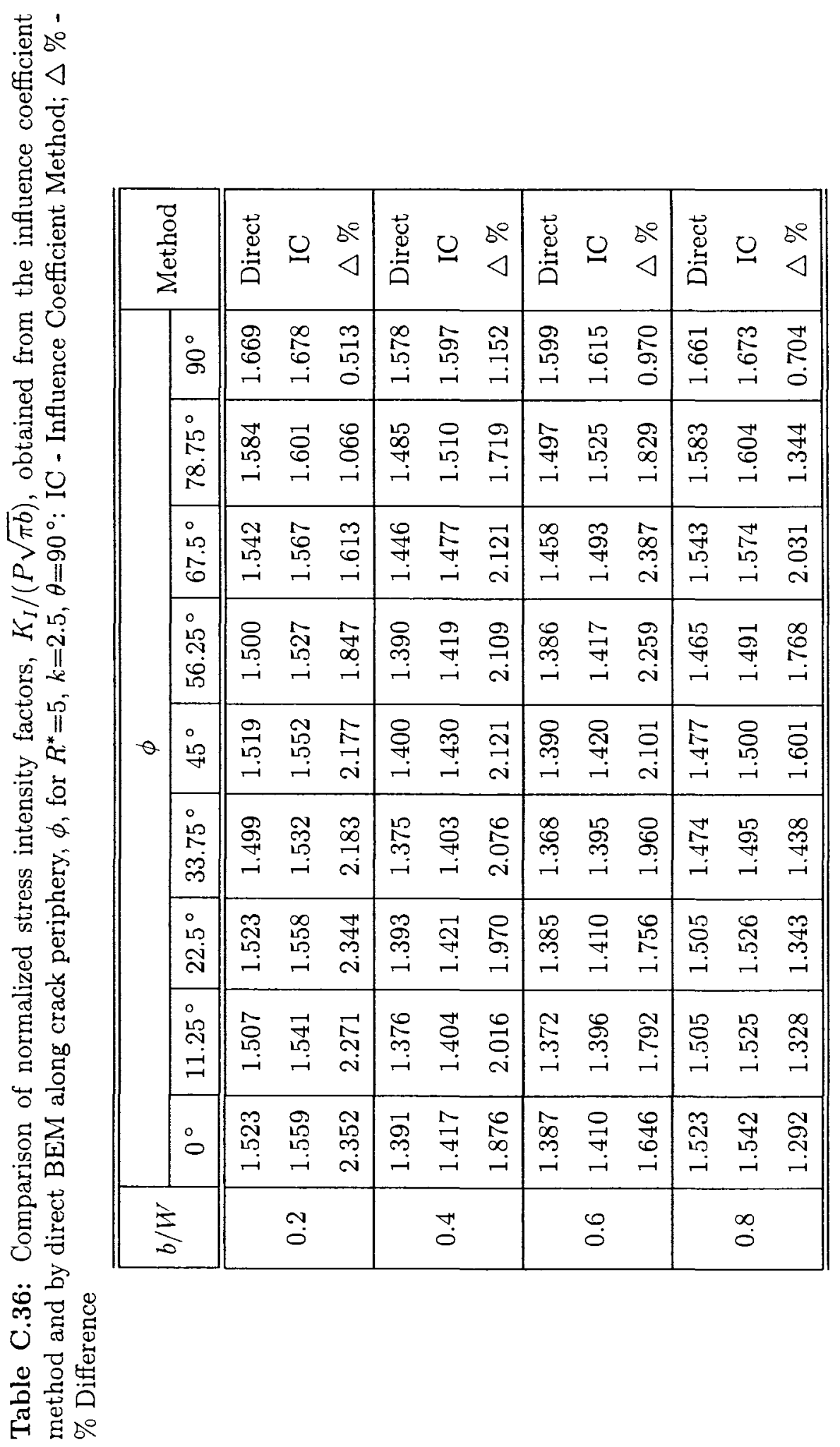




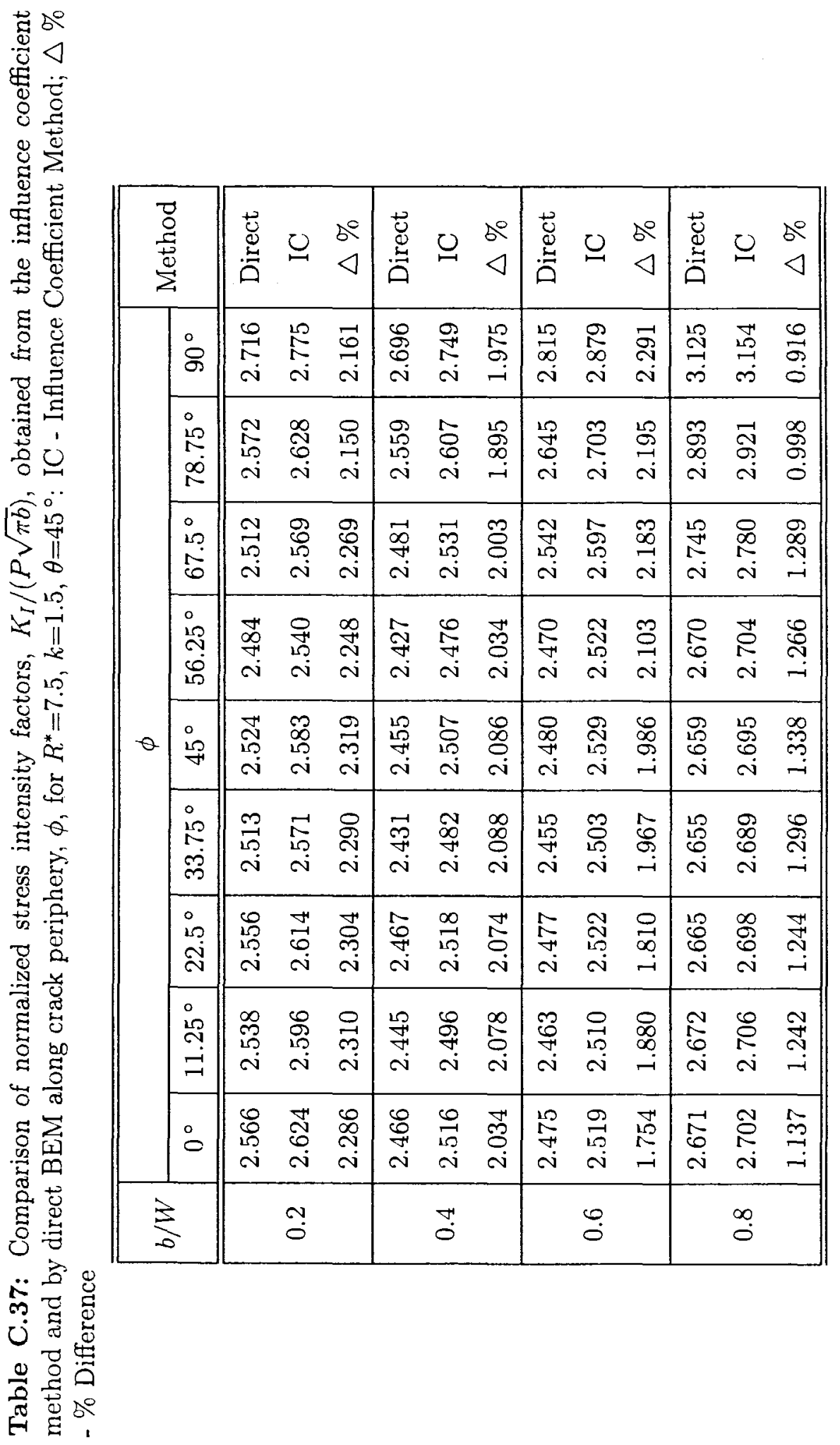




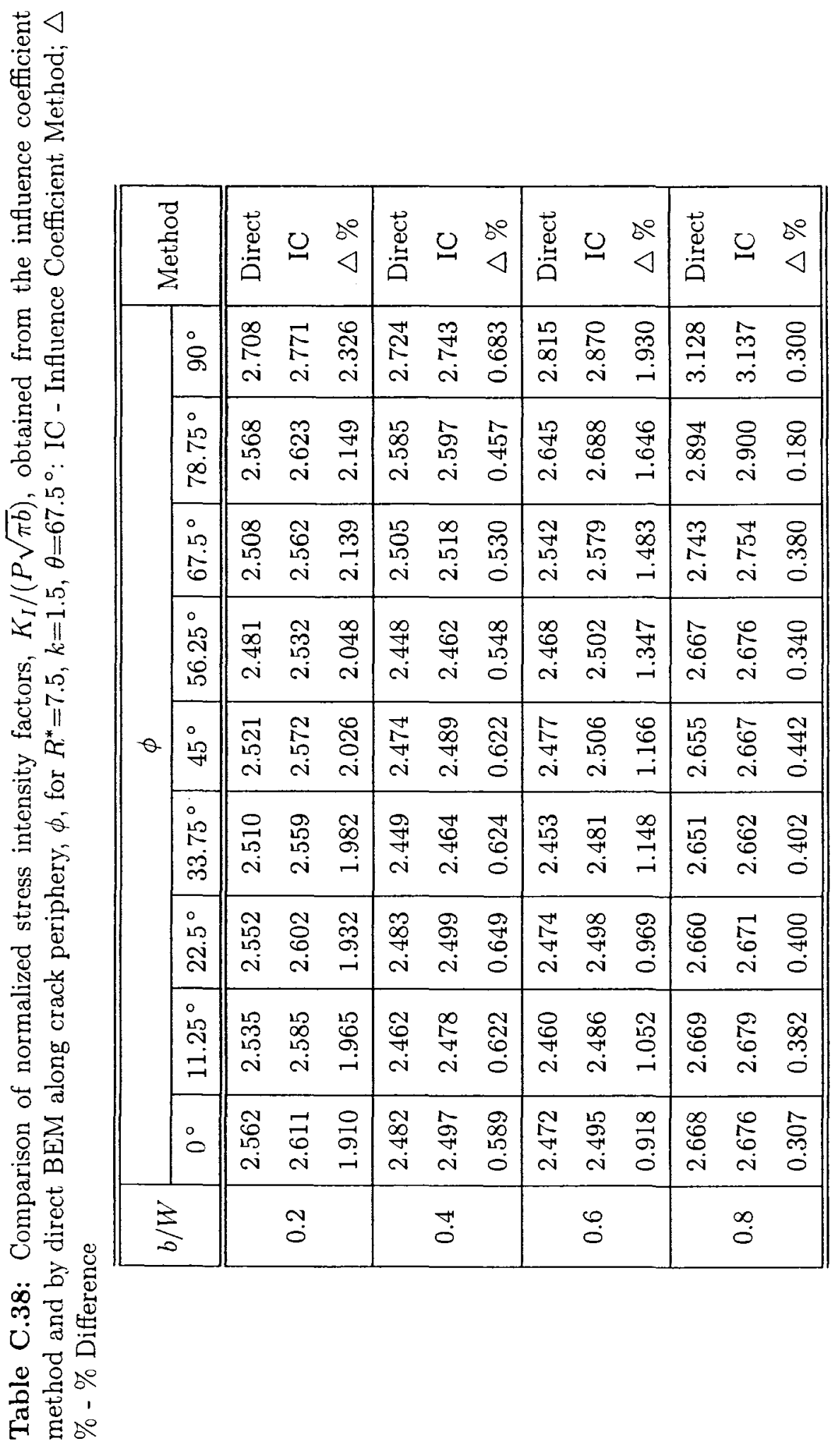




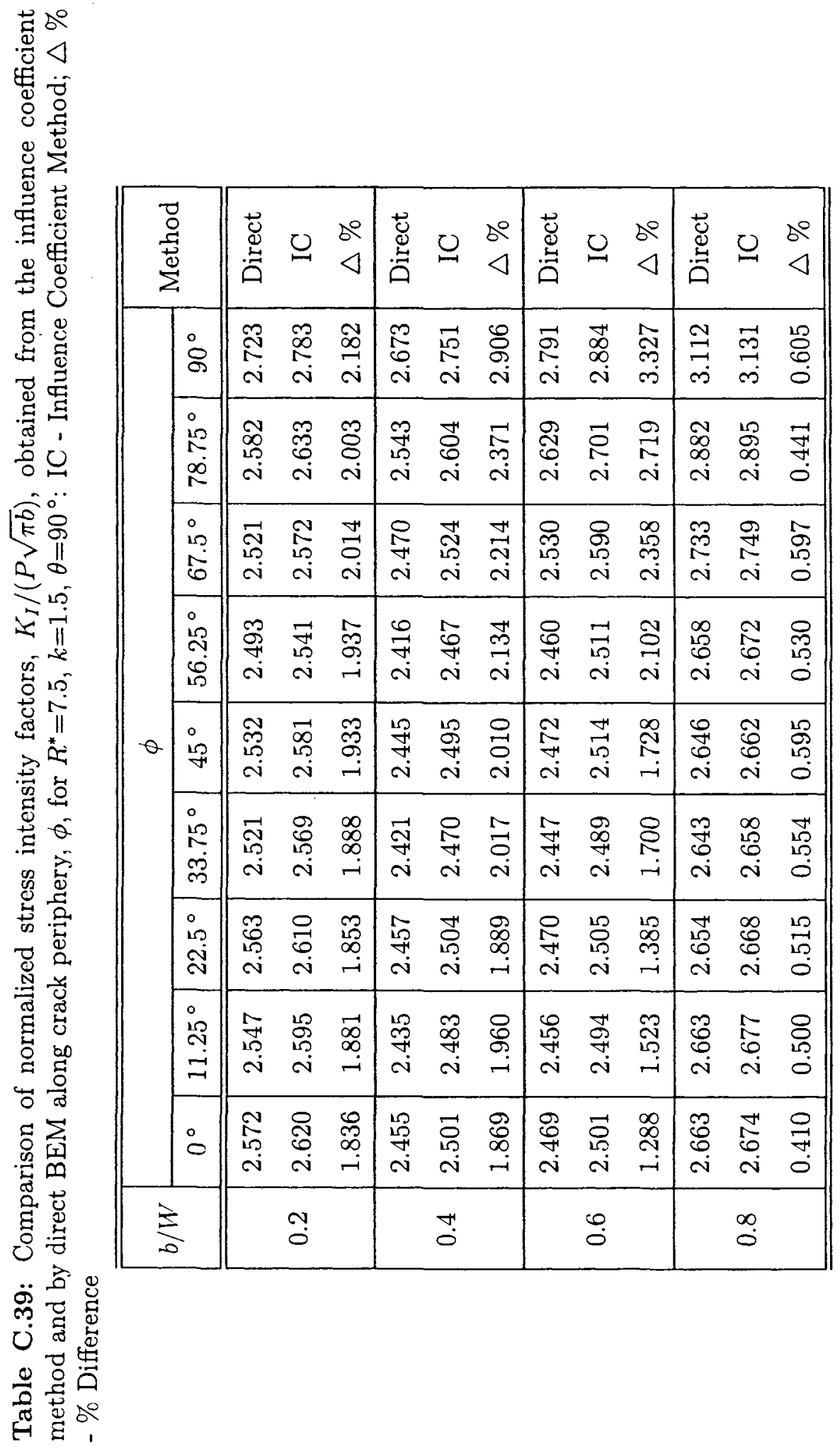




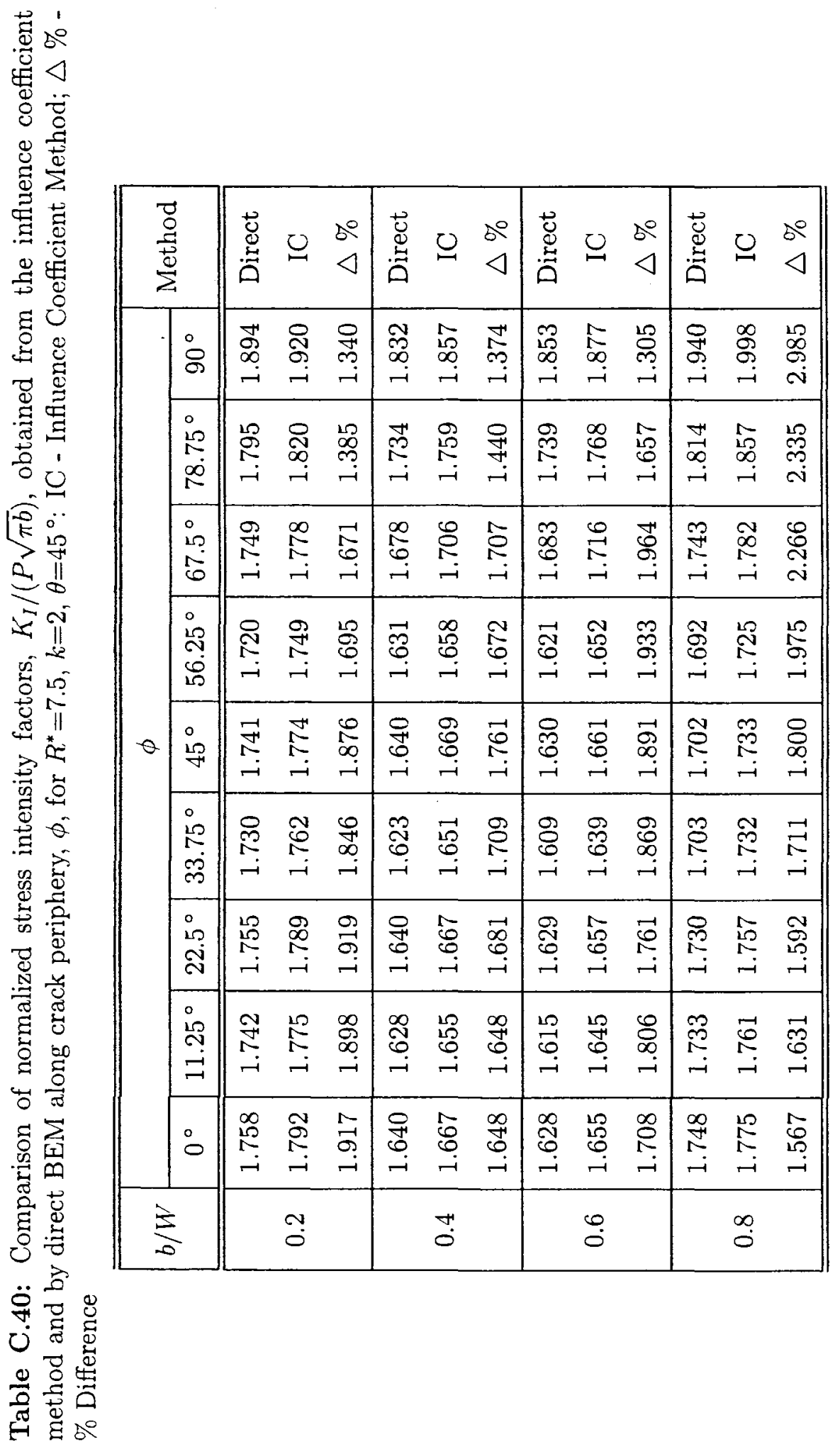




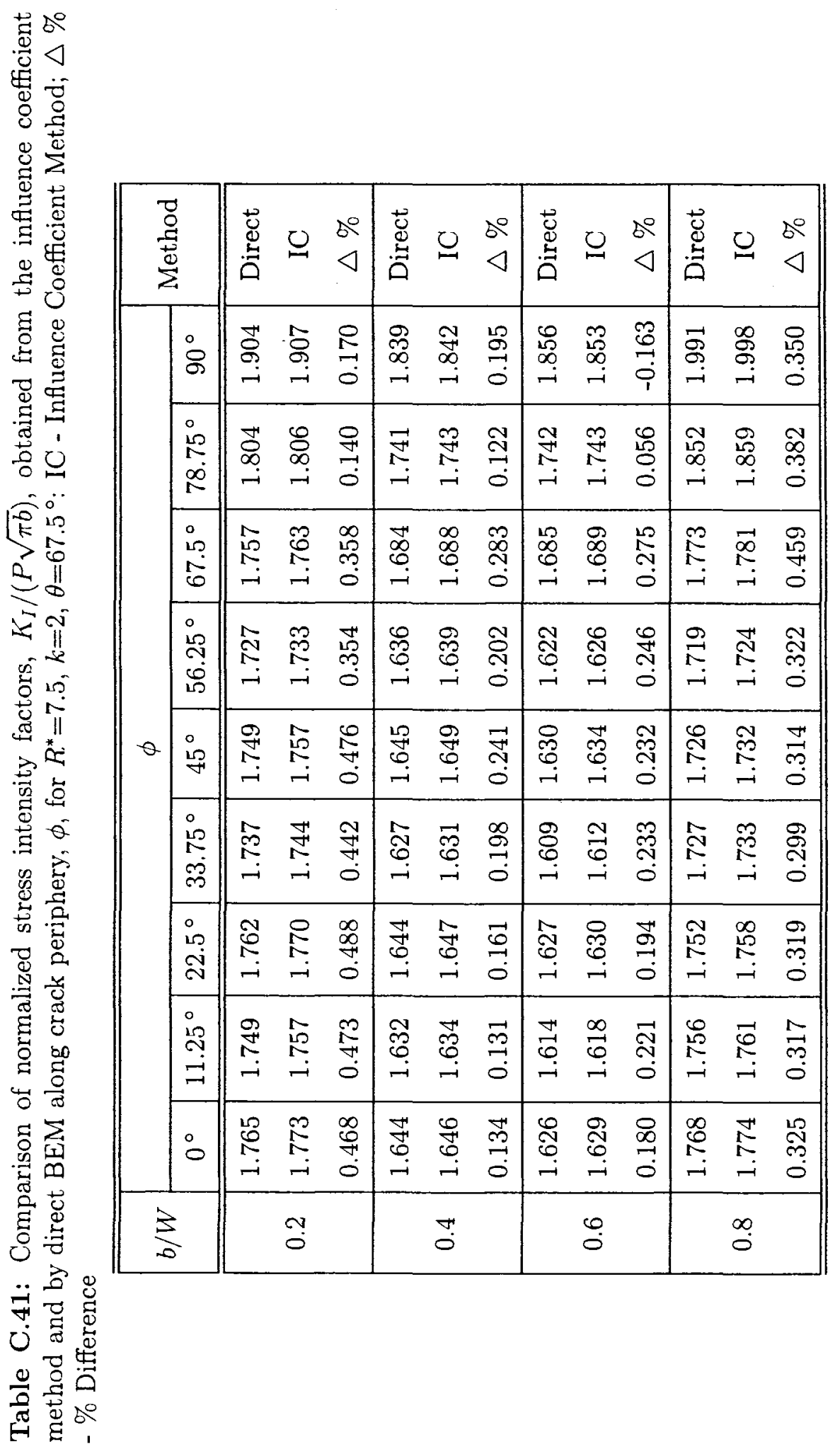




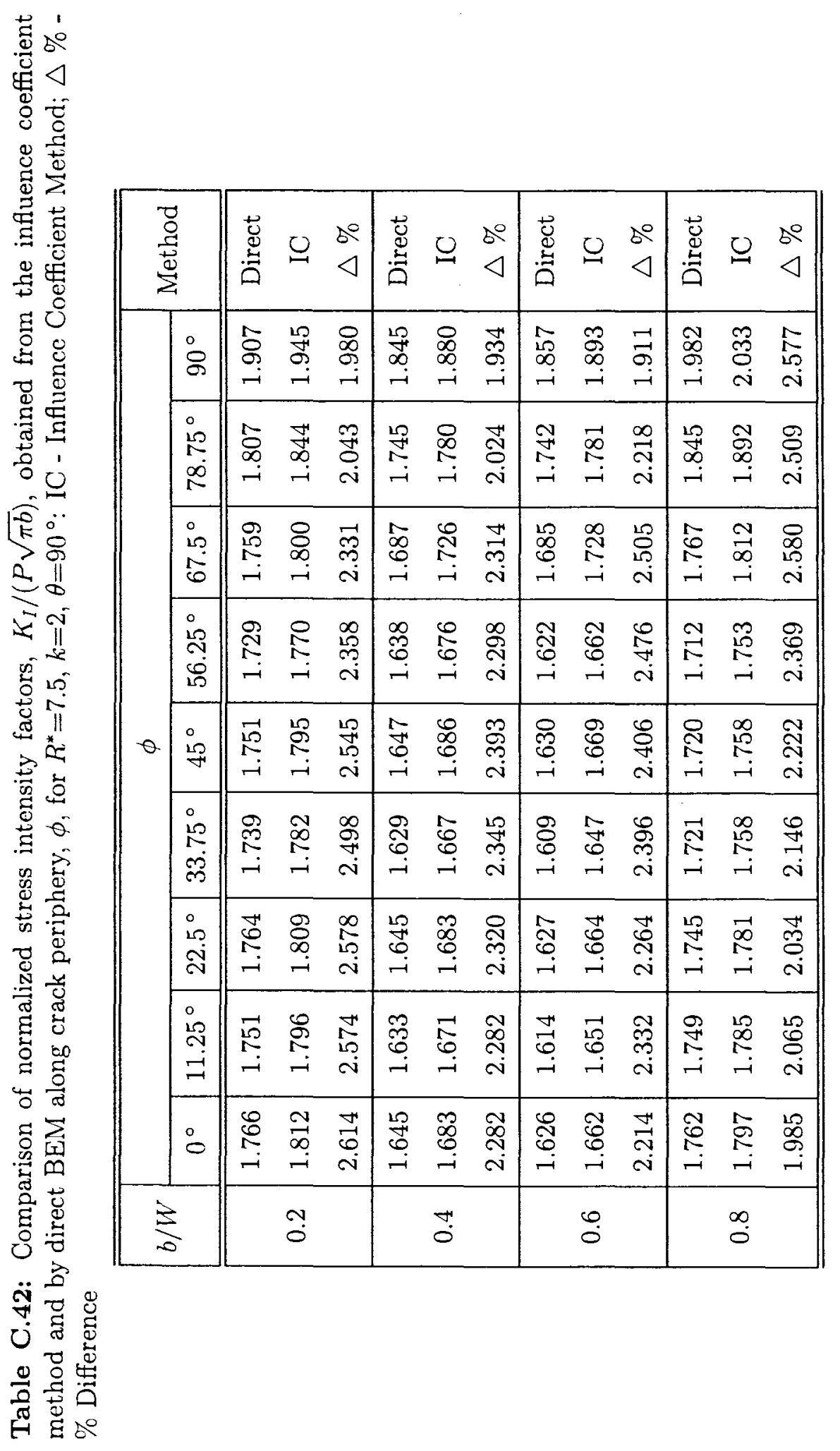




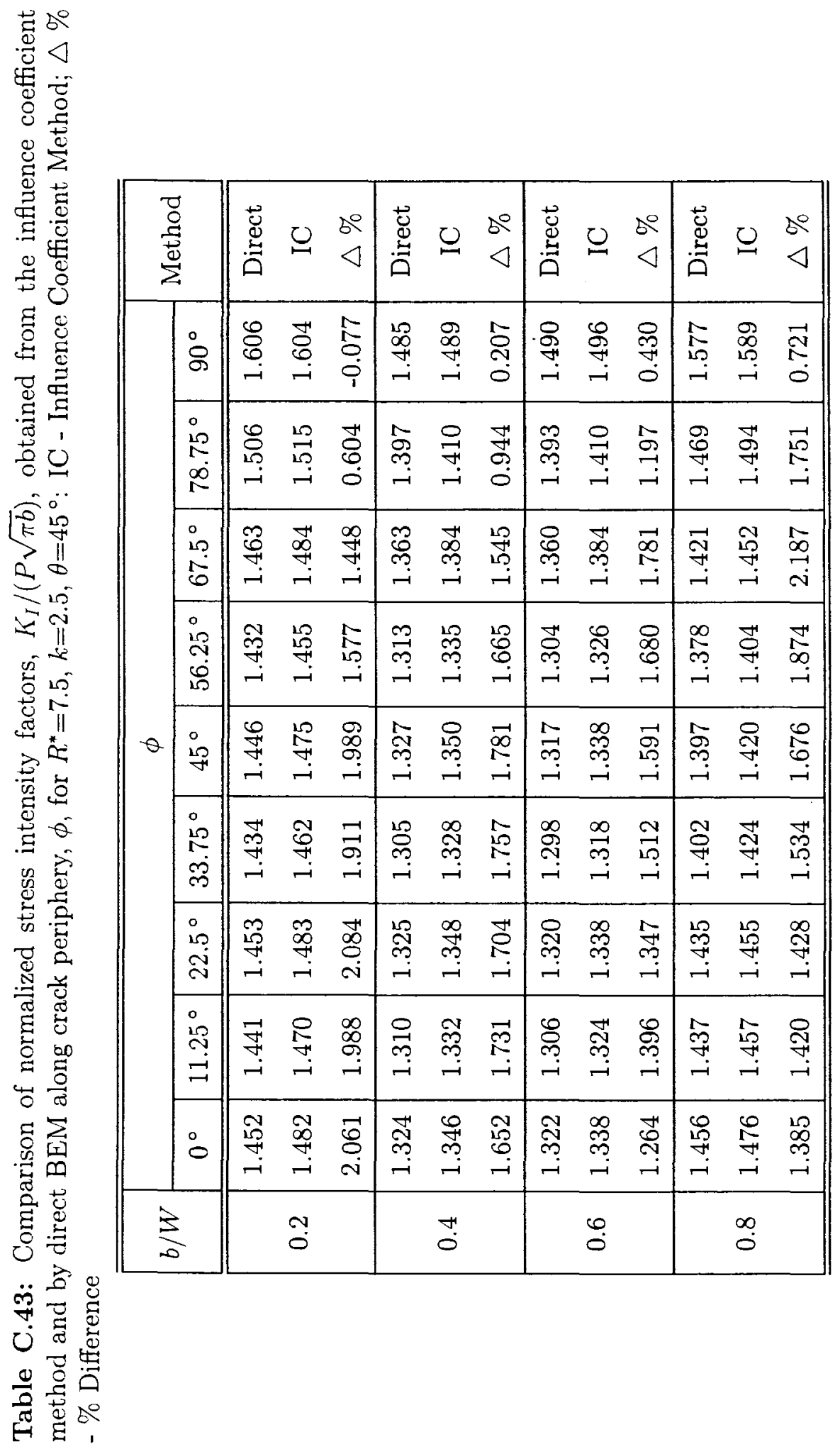




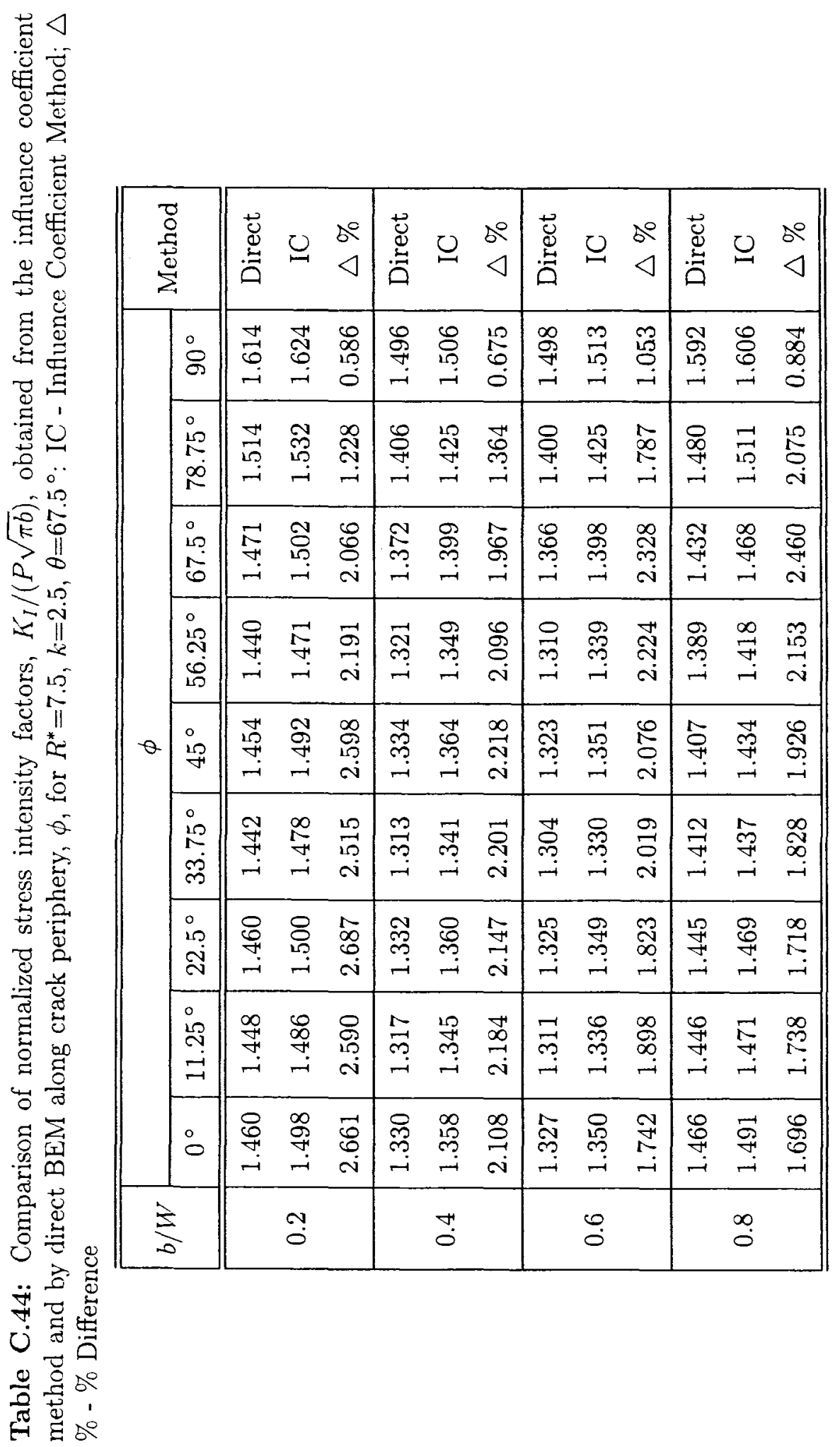




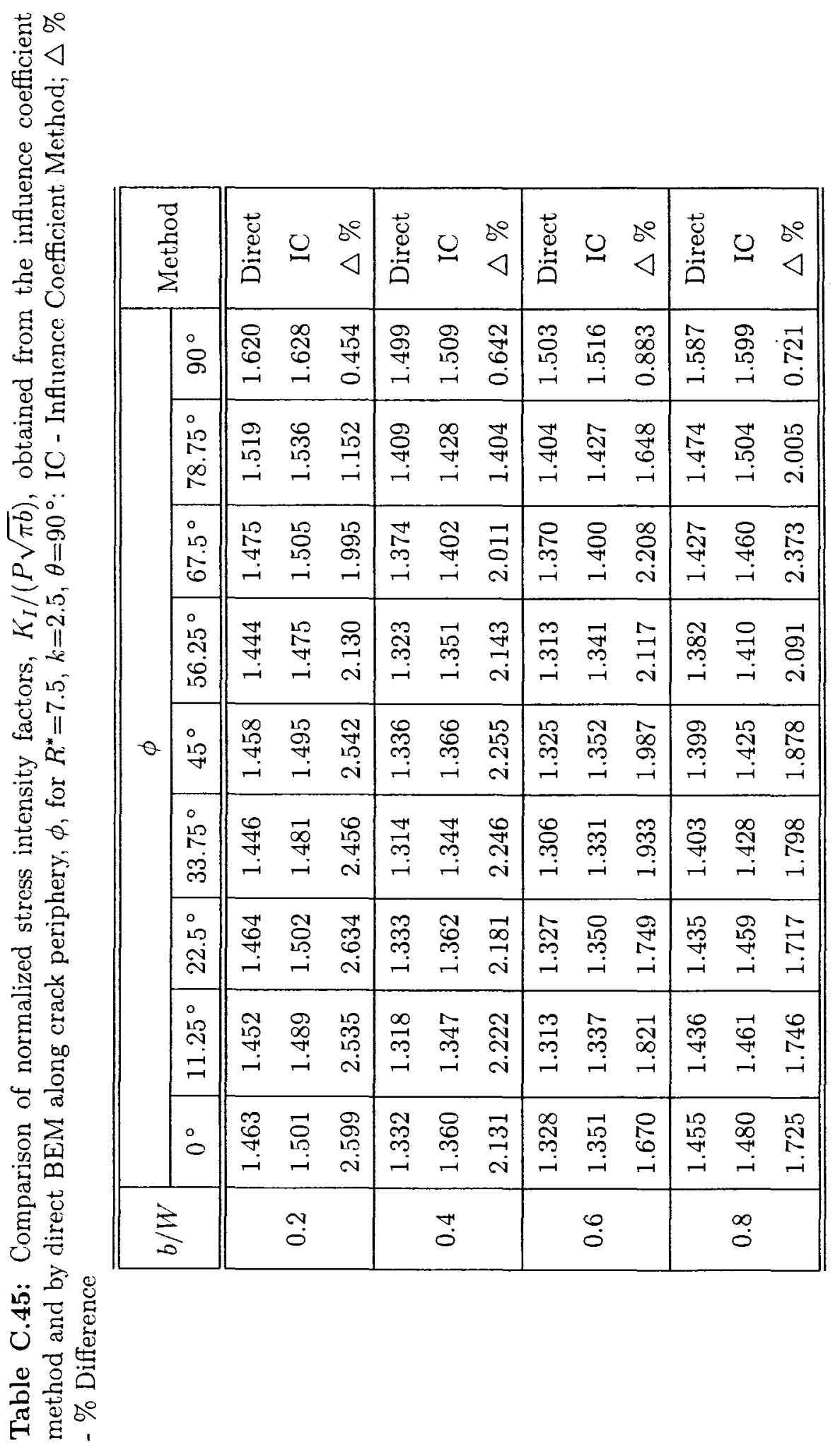




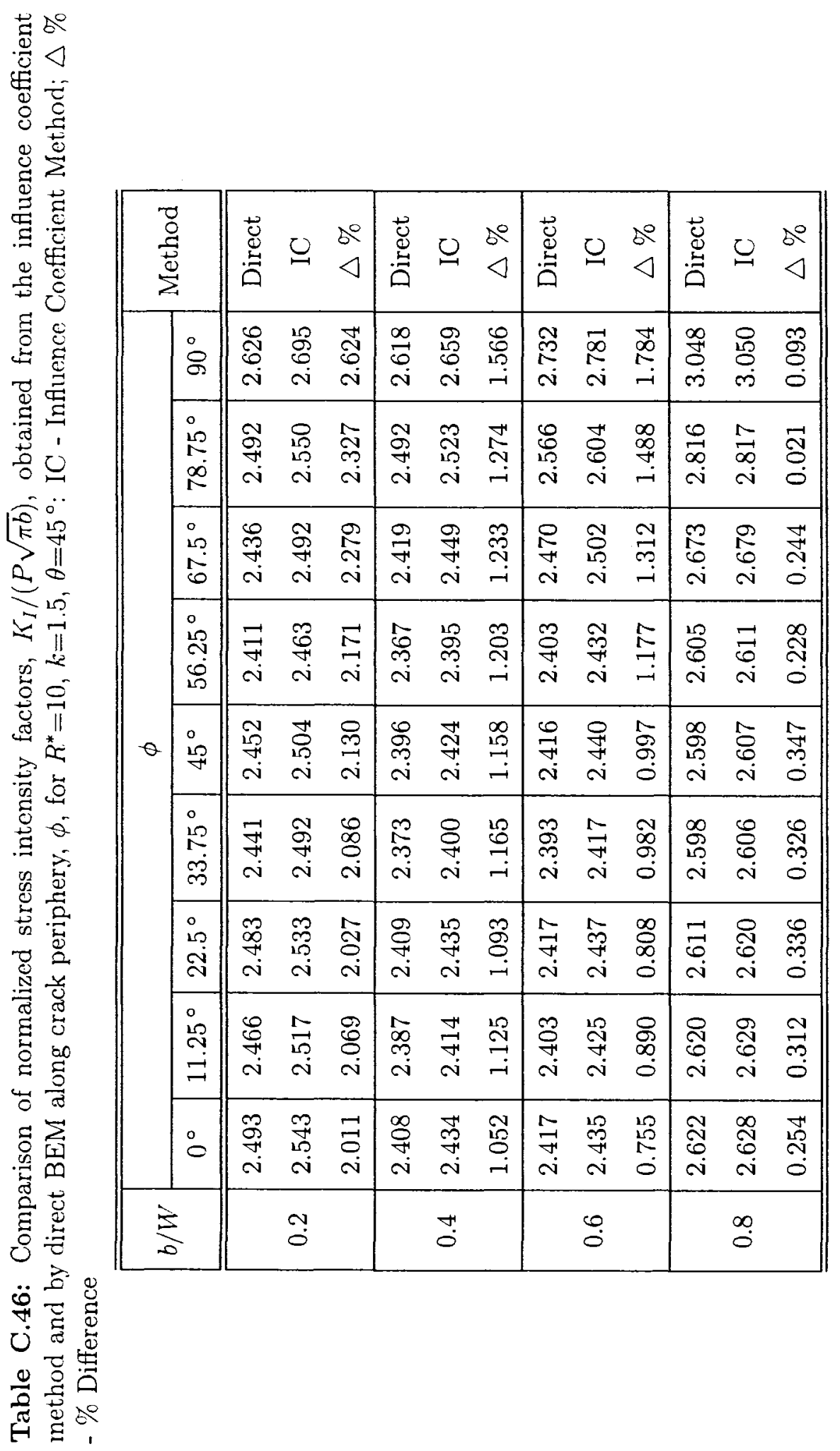




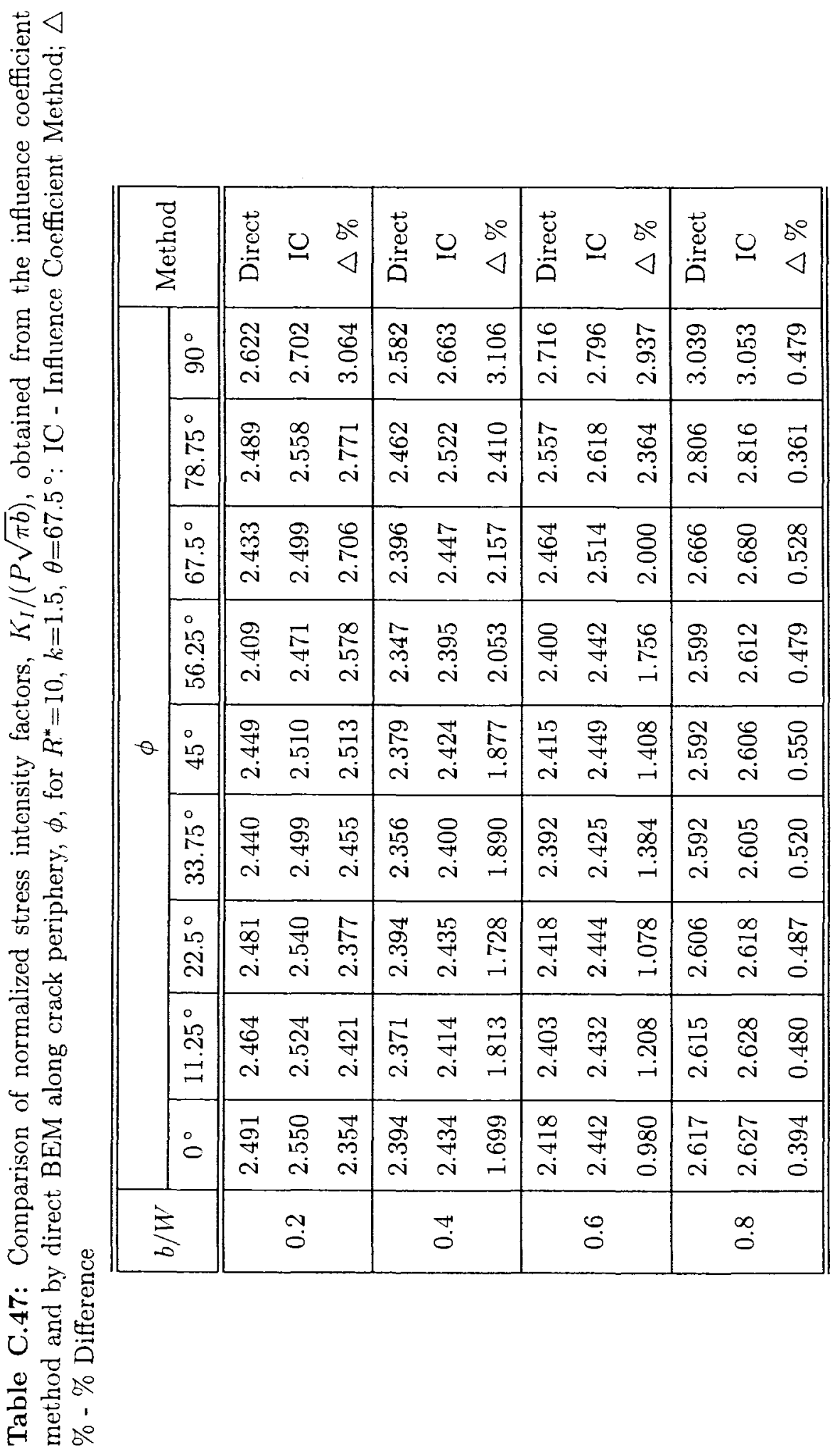




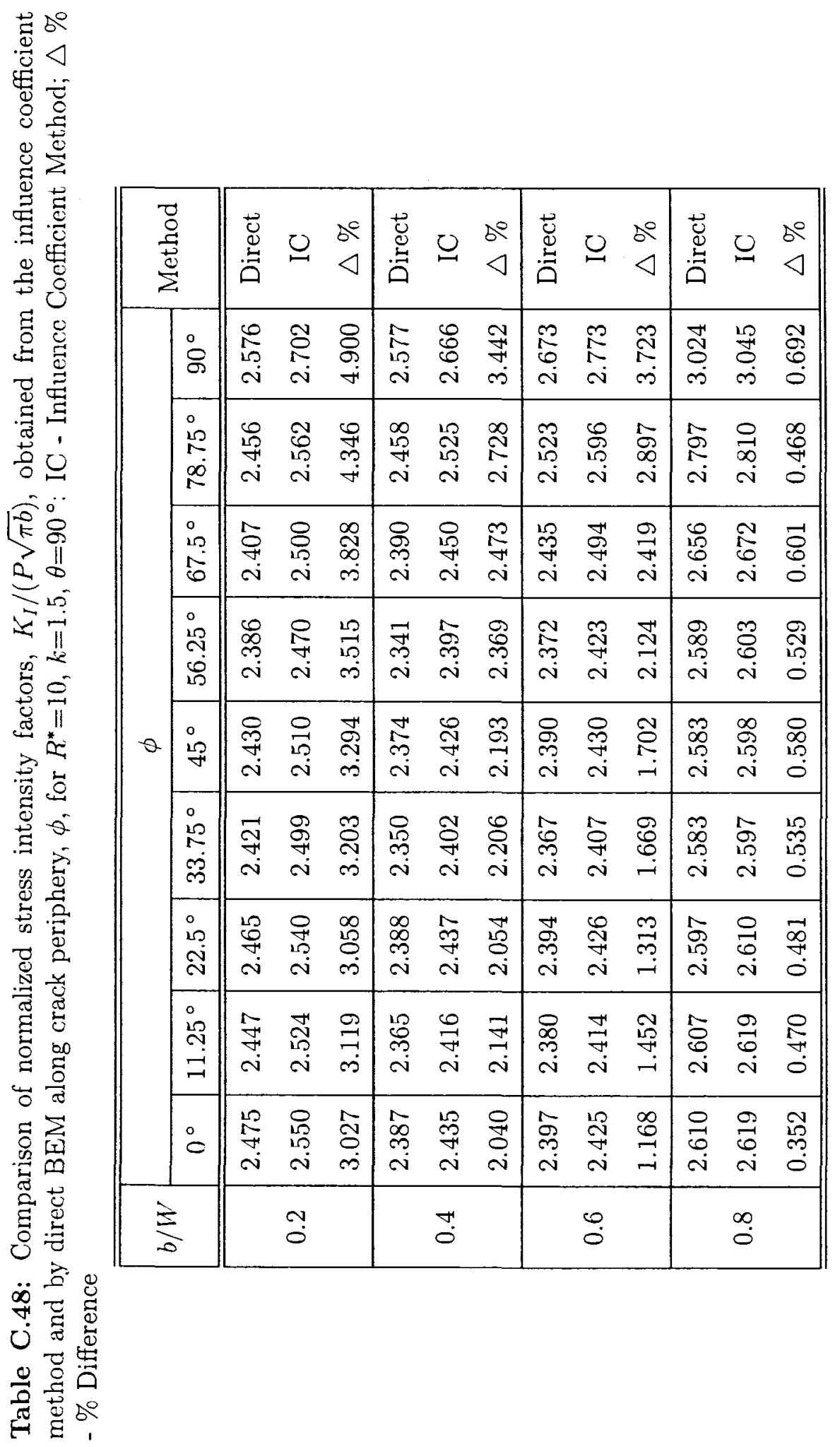




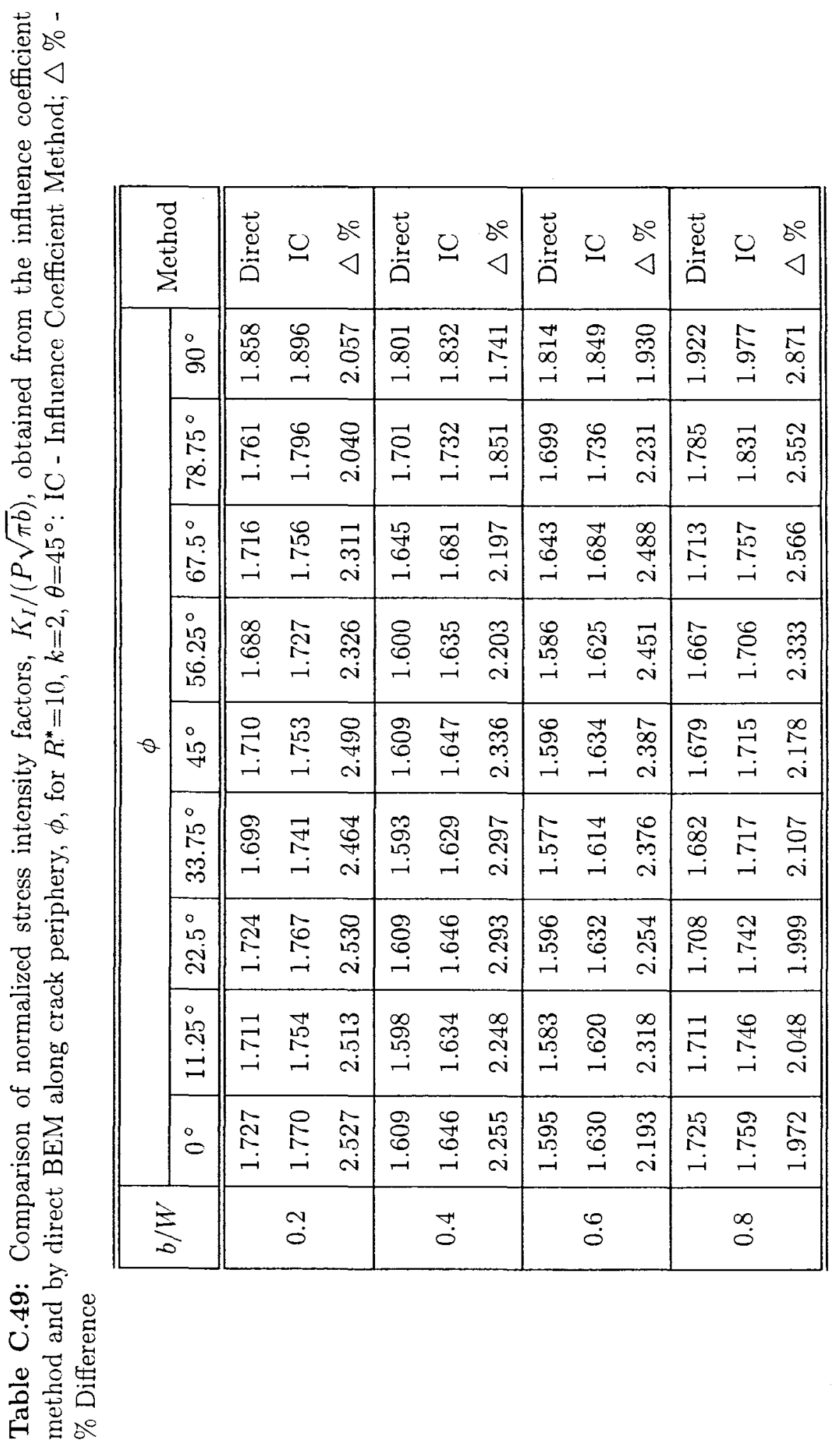




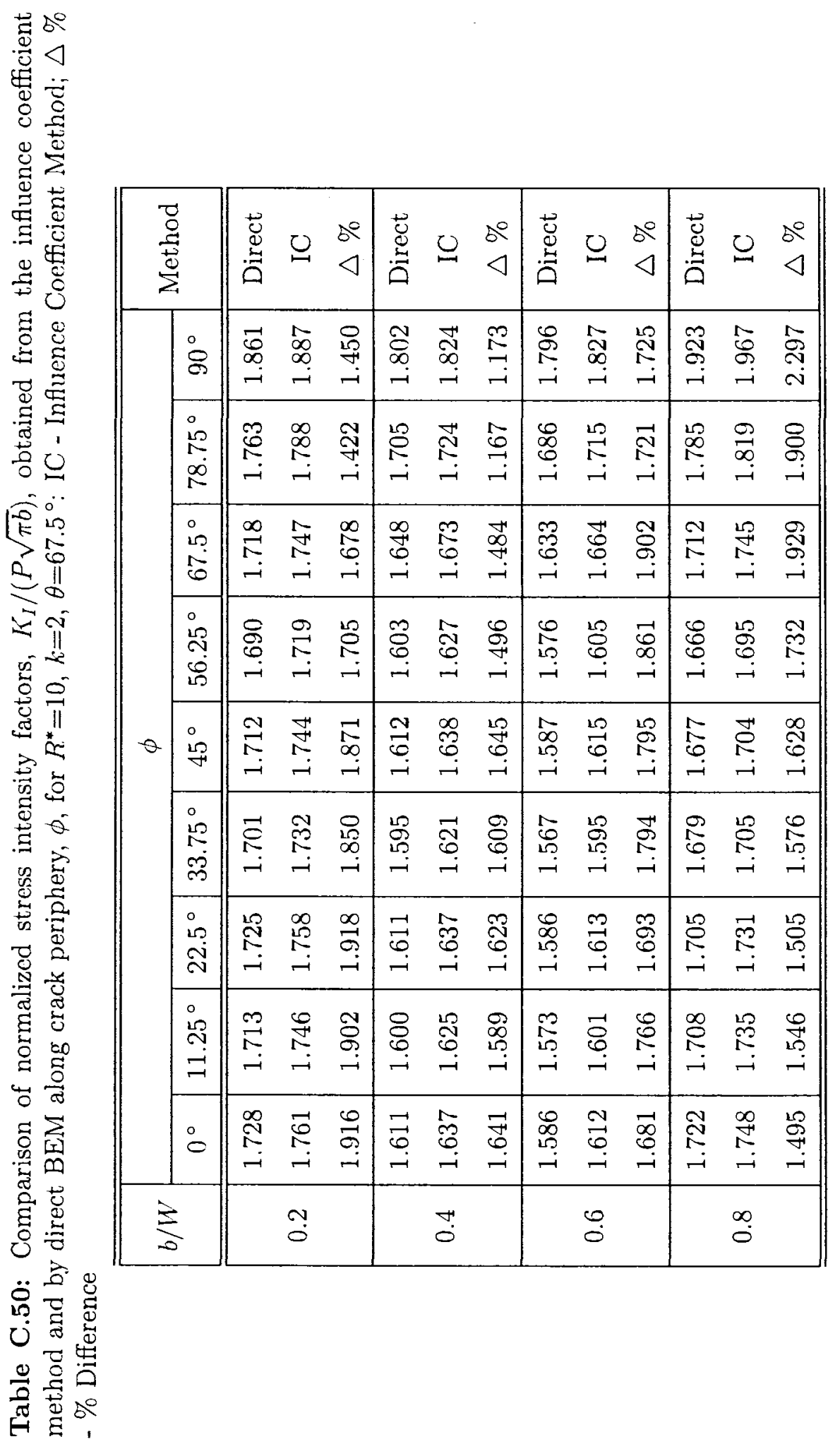




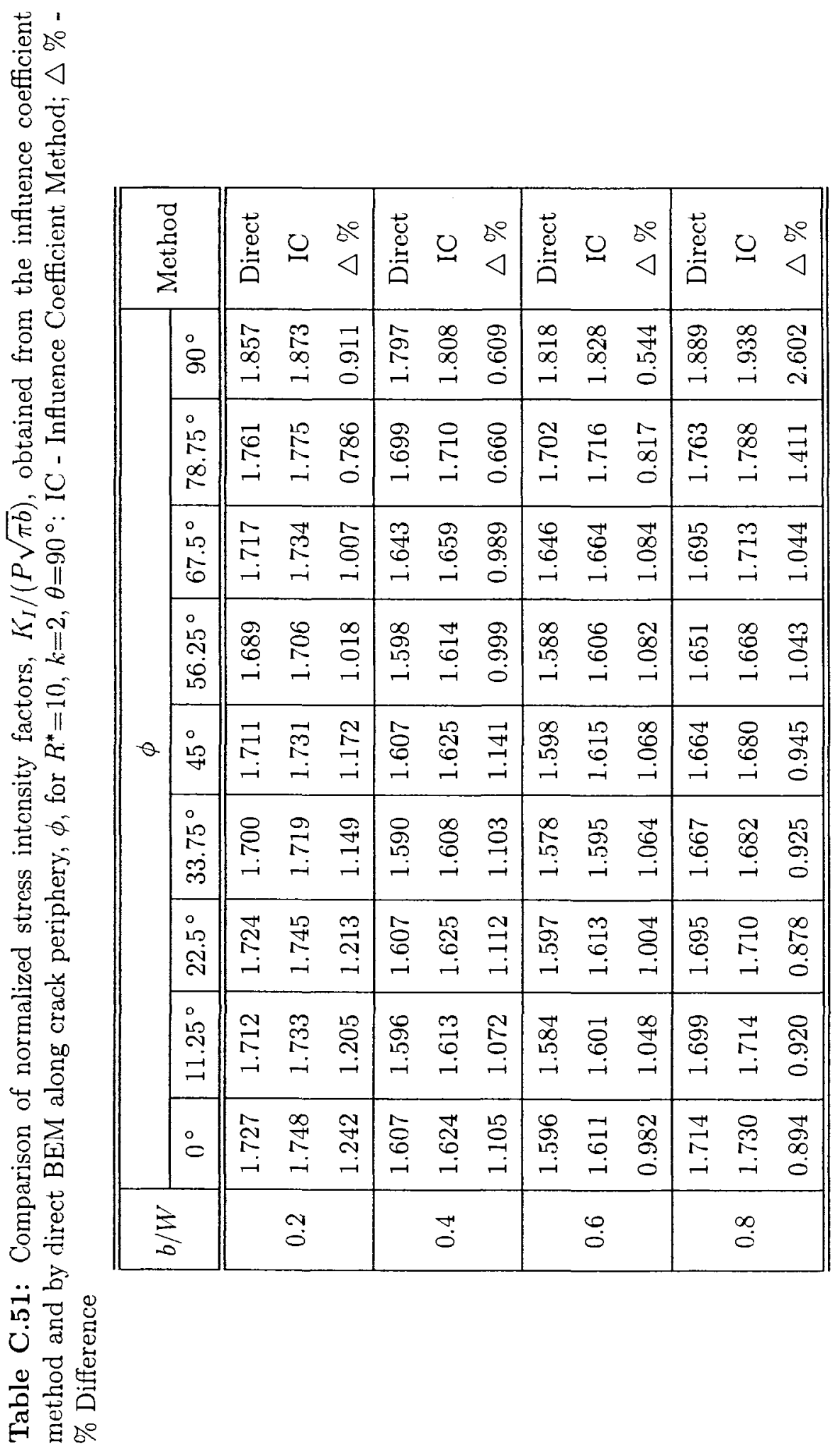




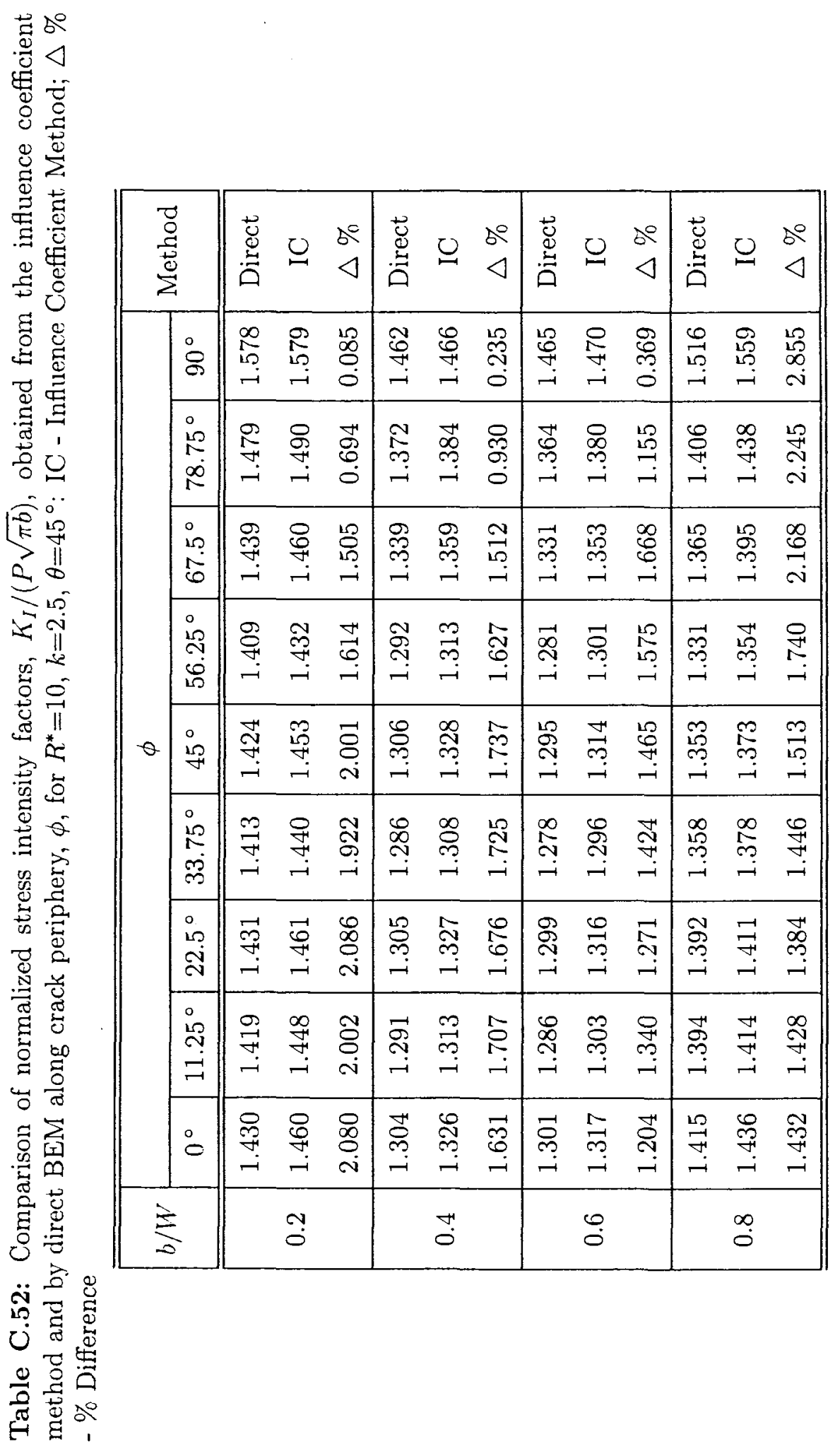




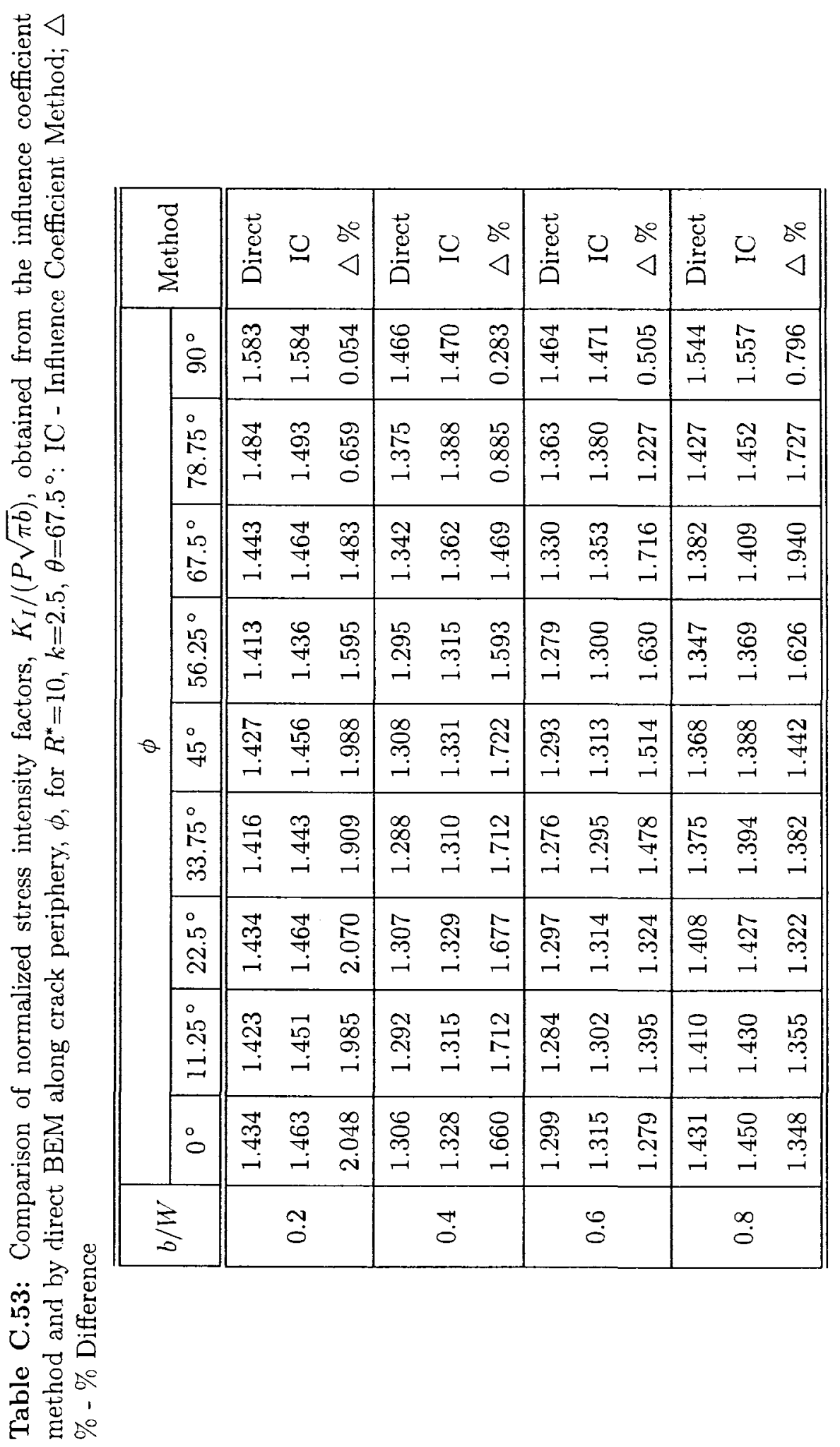




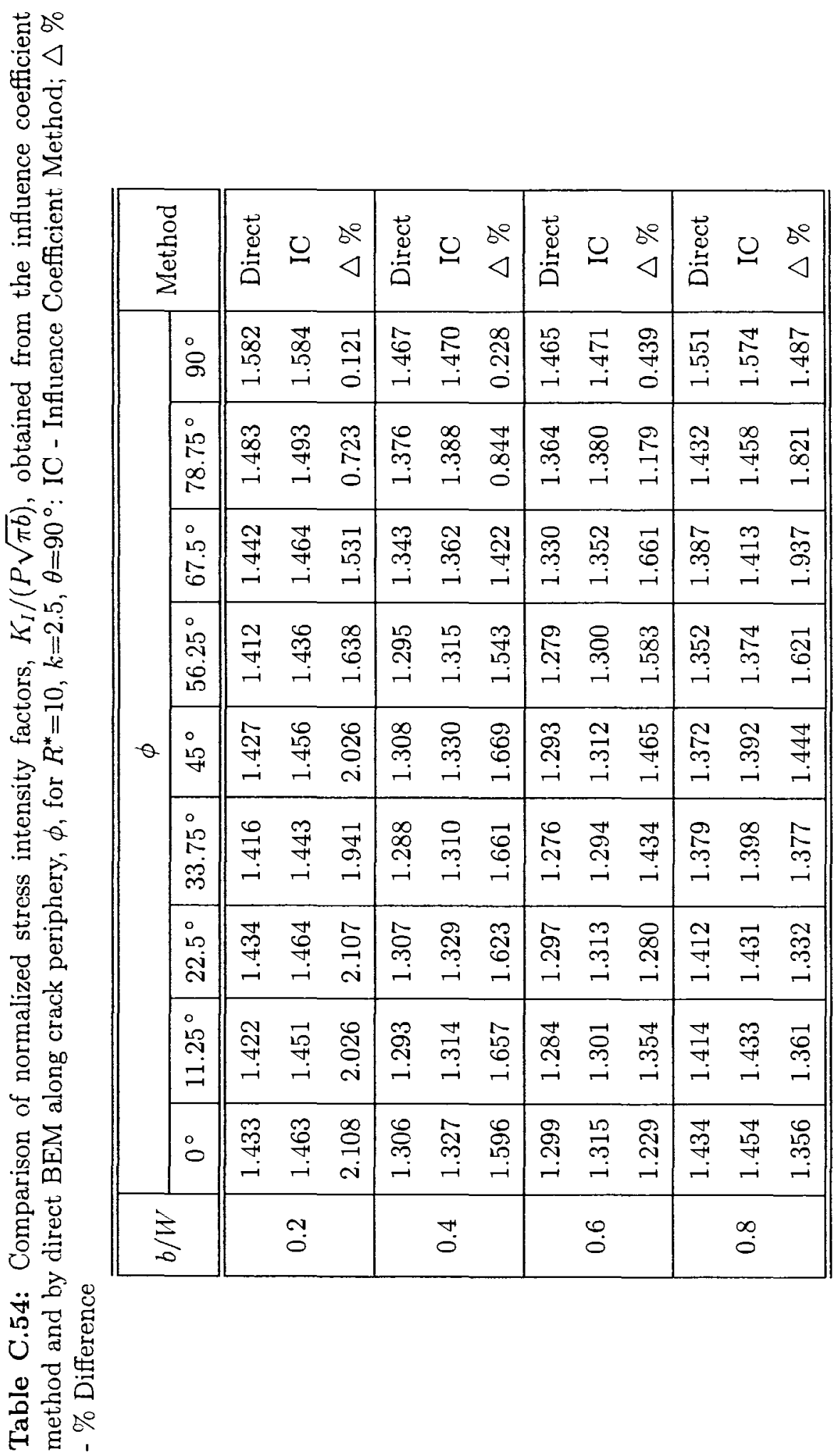


Appendix D

Stress Intensity Factors due to Residual

Stresses from Autofrettage 


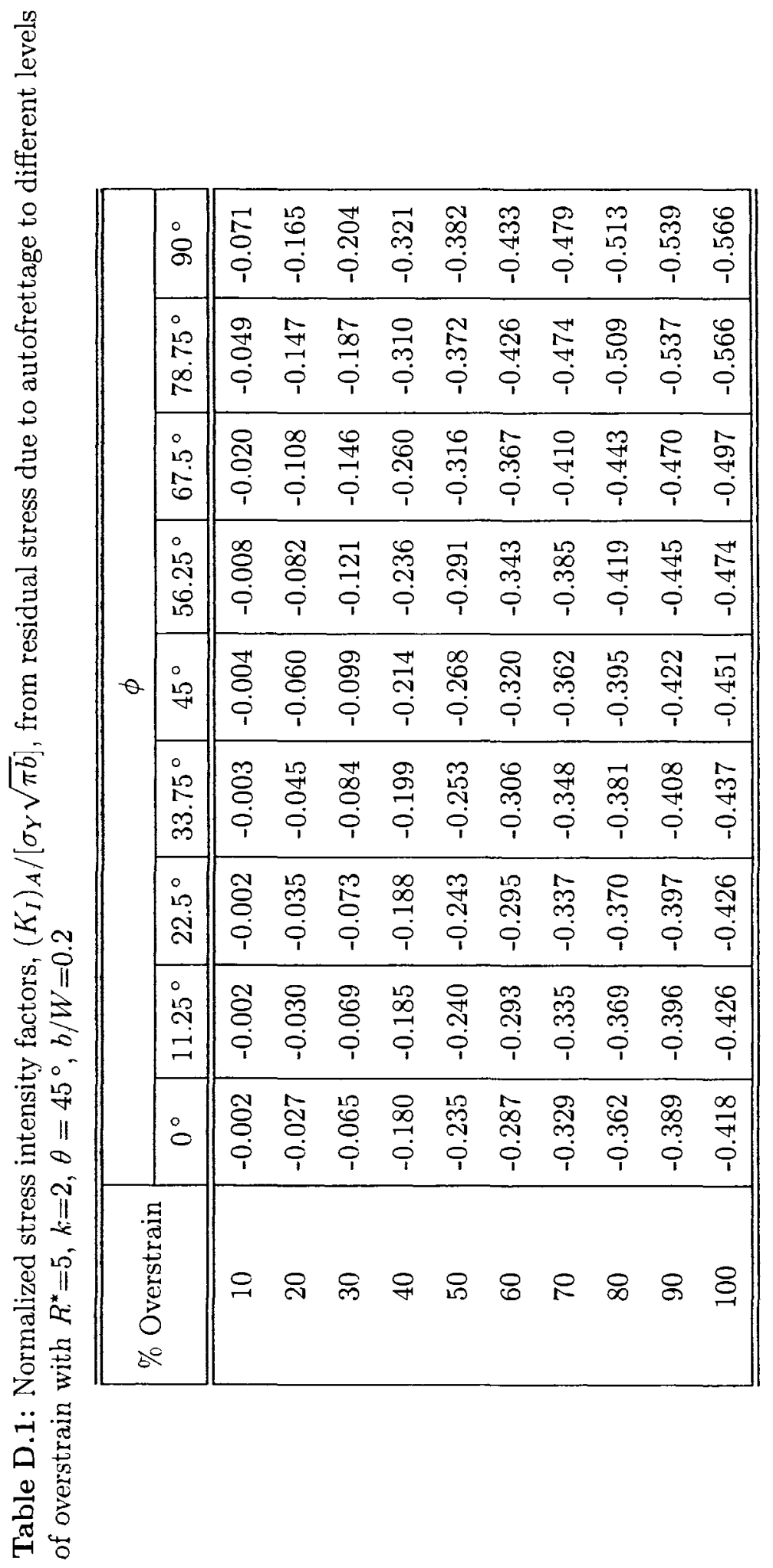




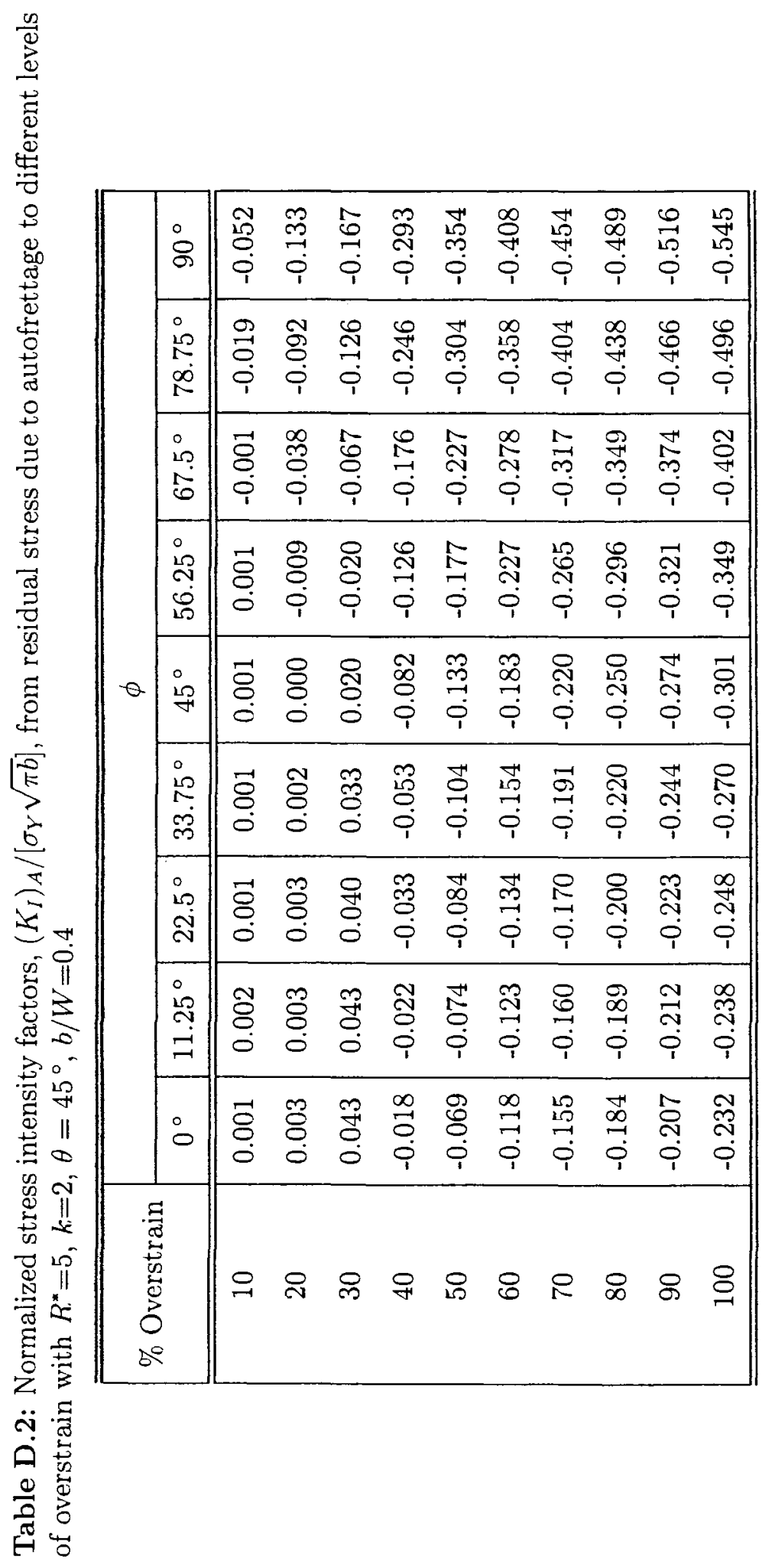




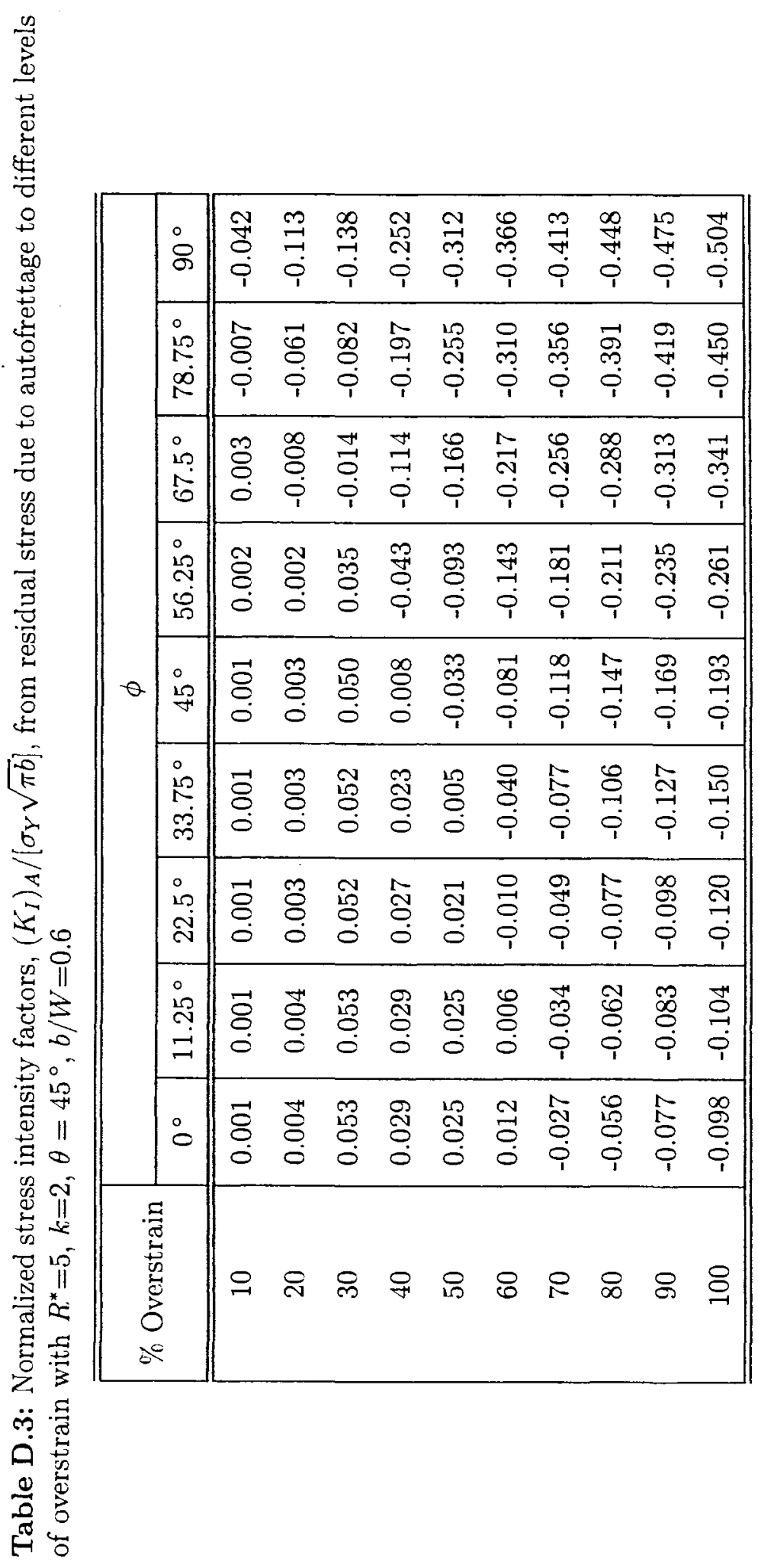




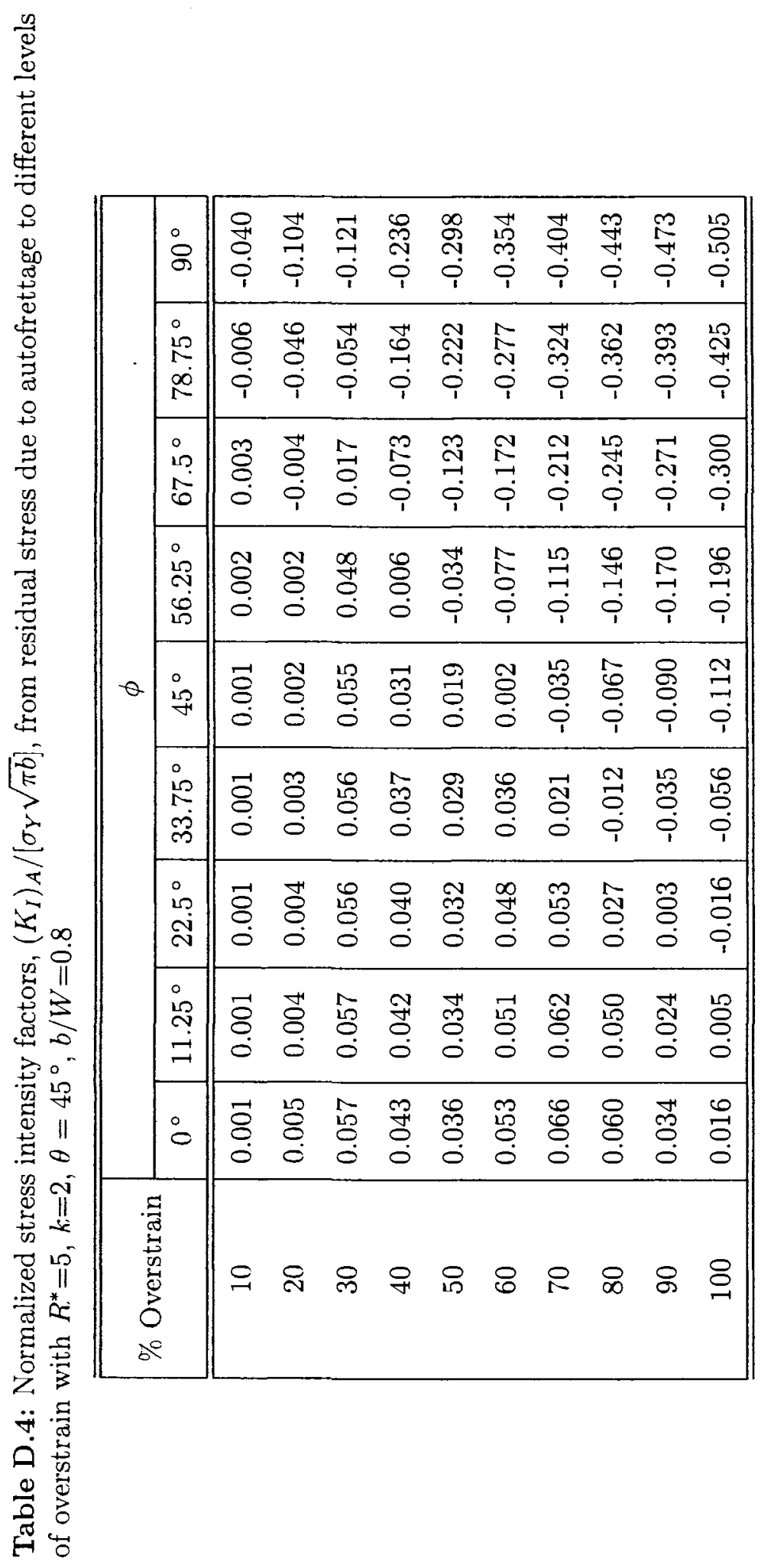




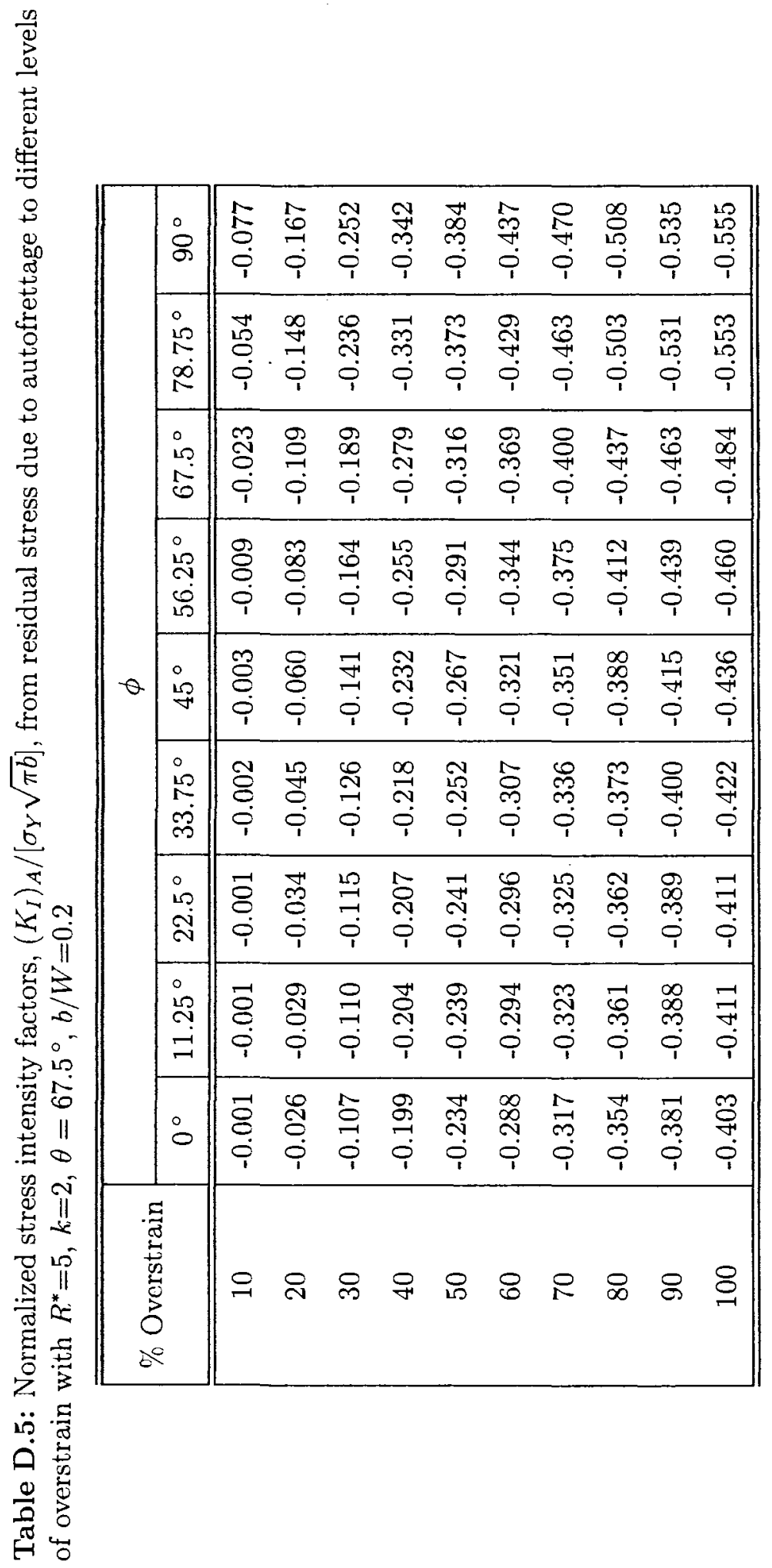




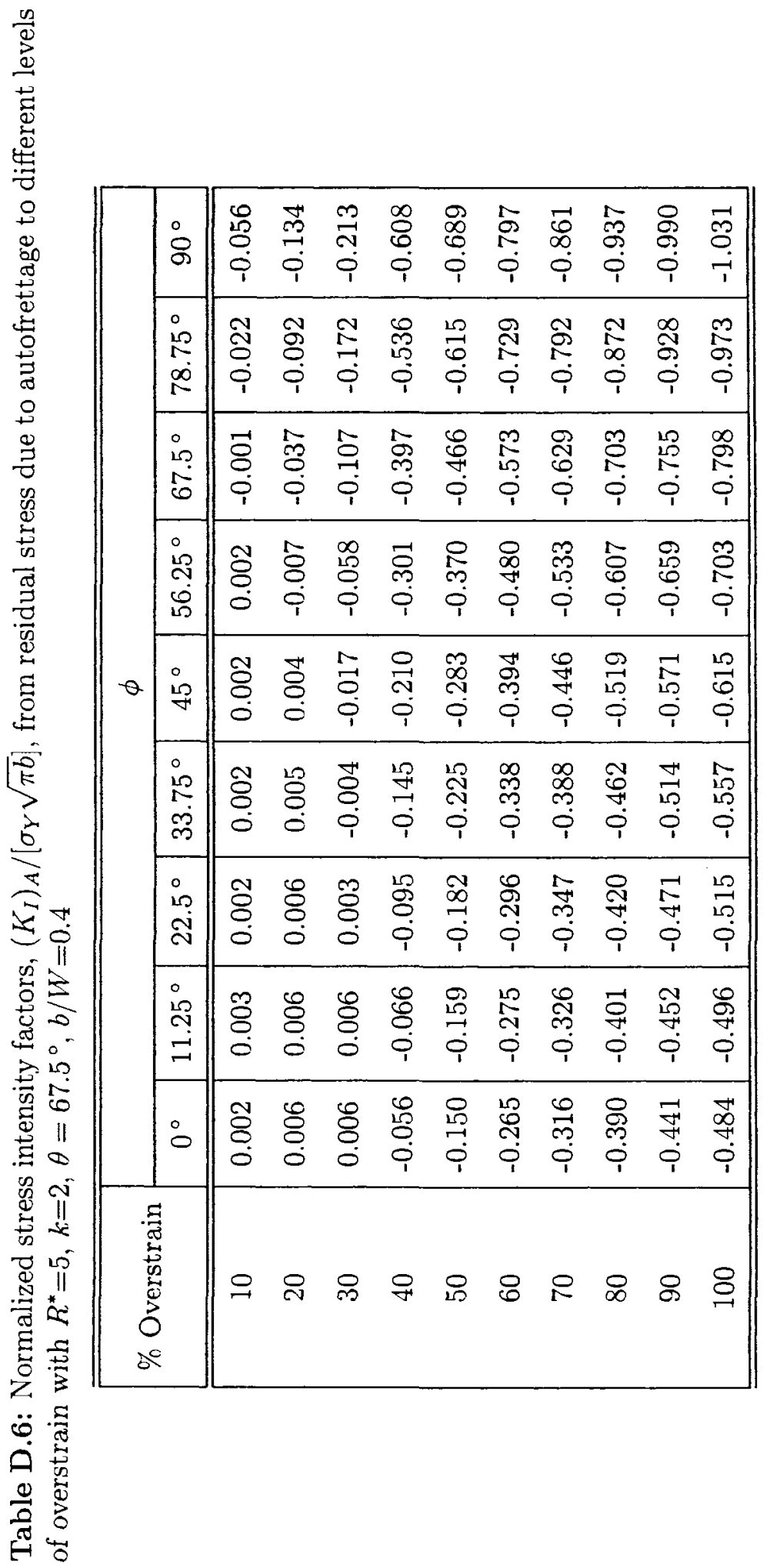




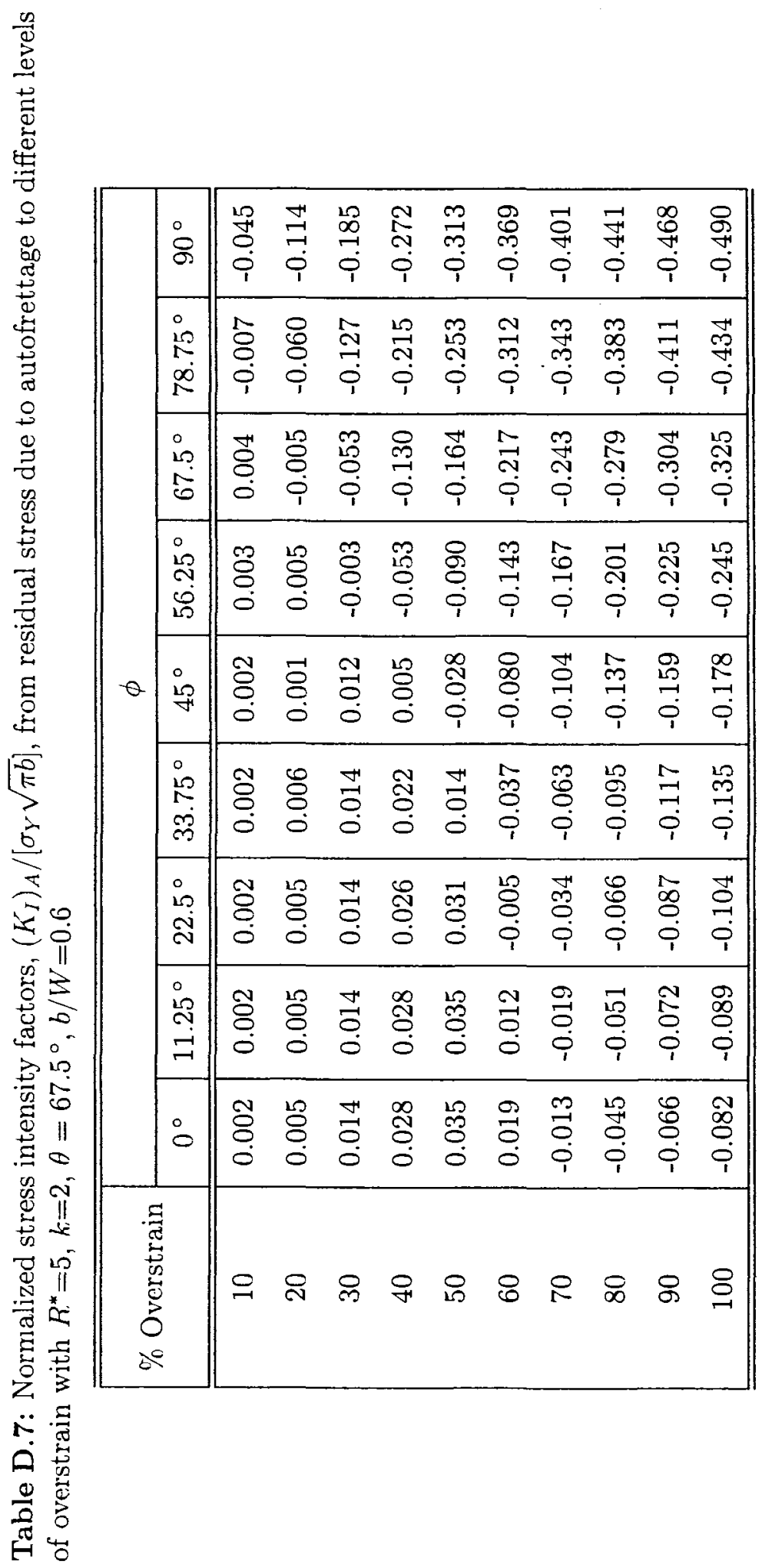




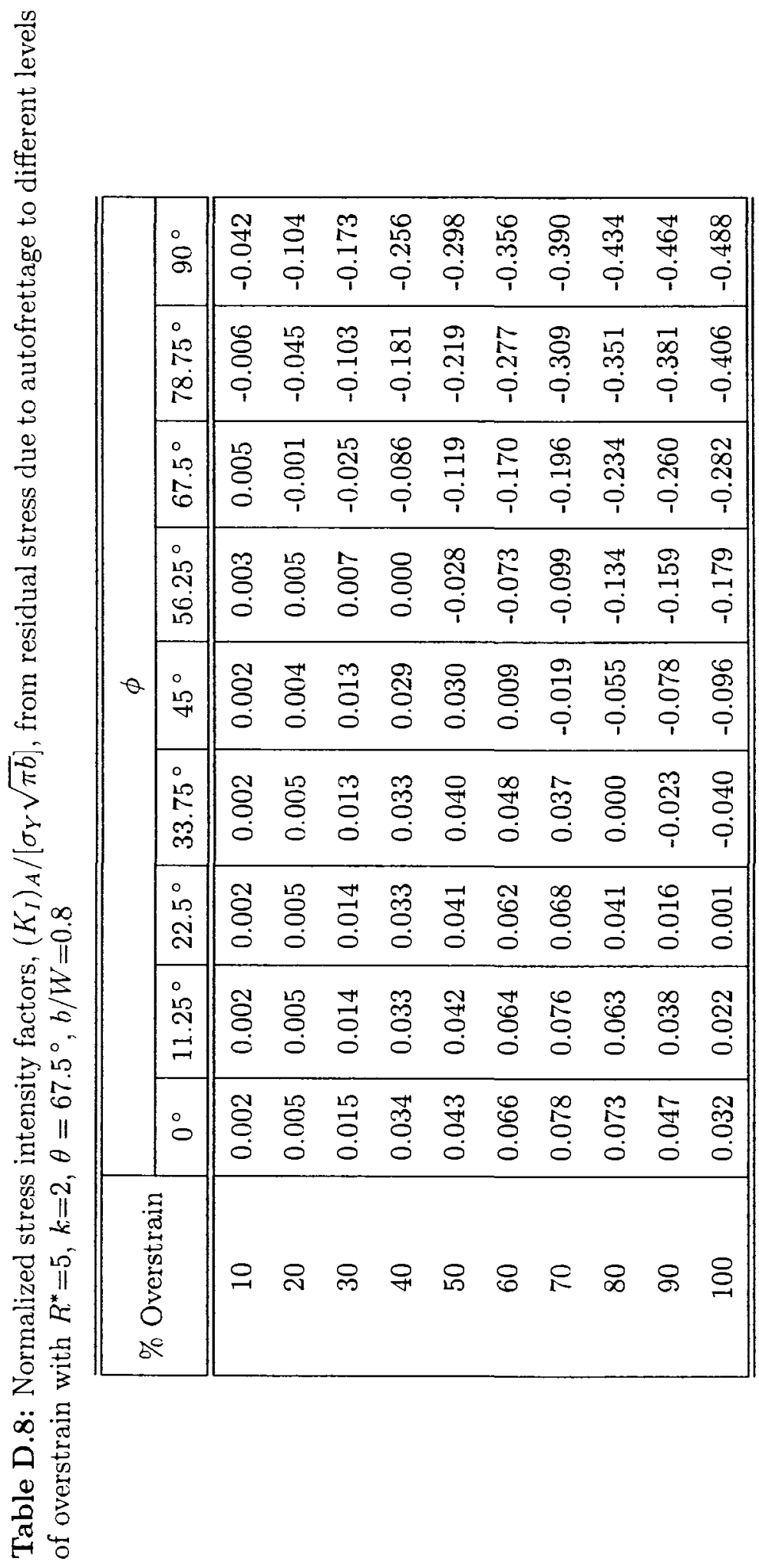




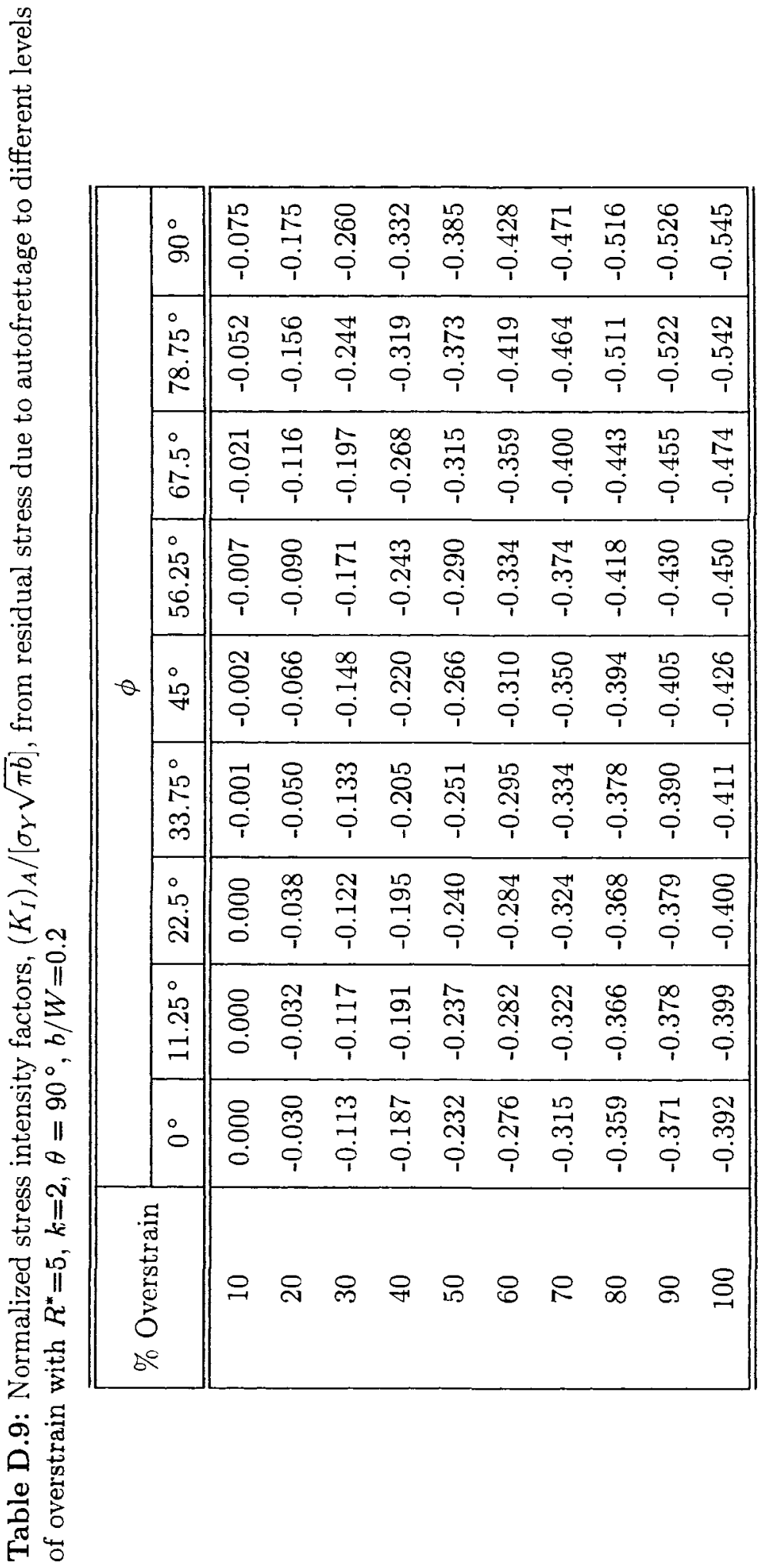




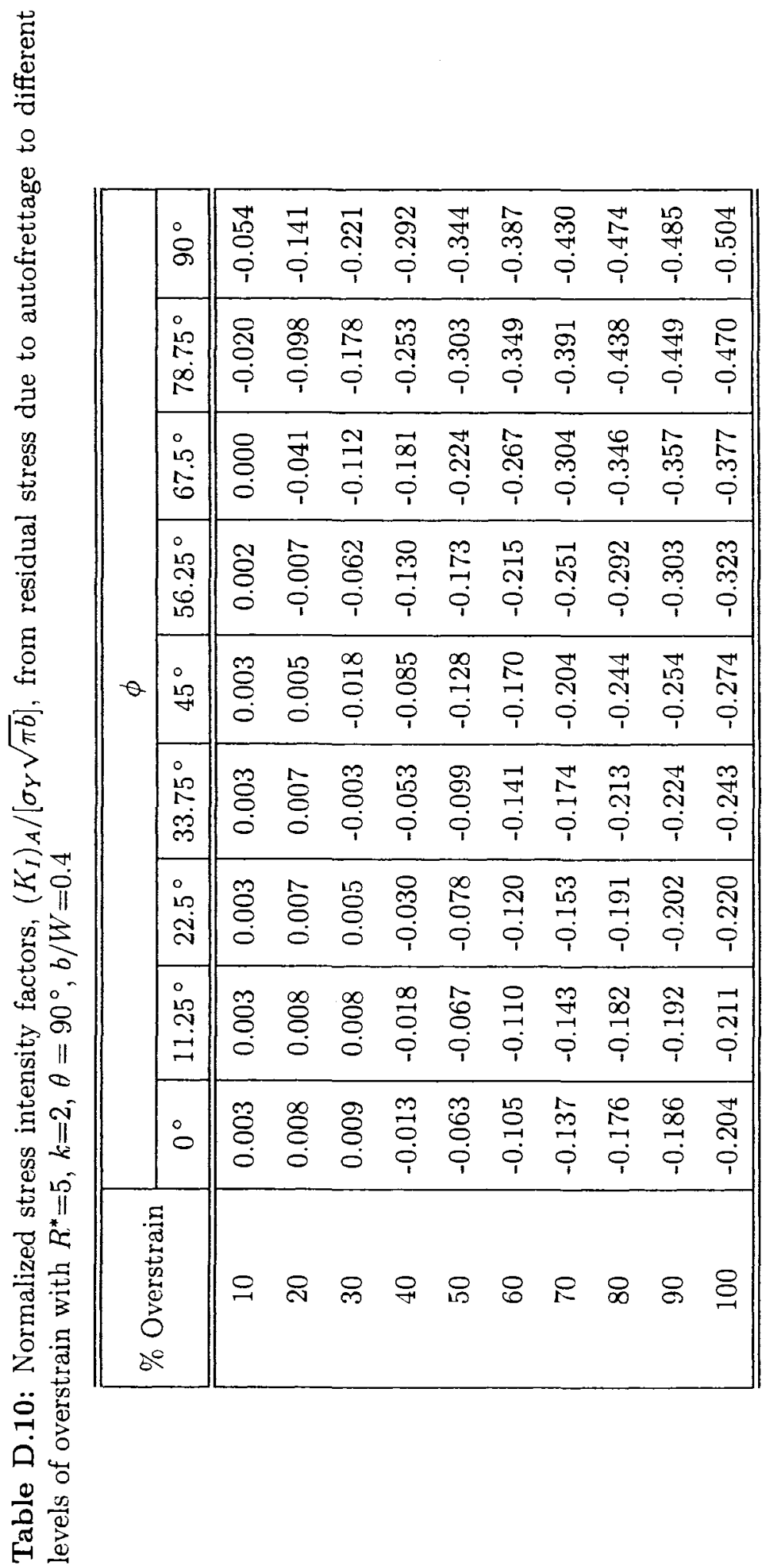




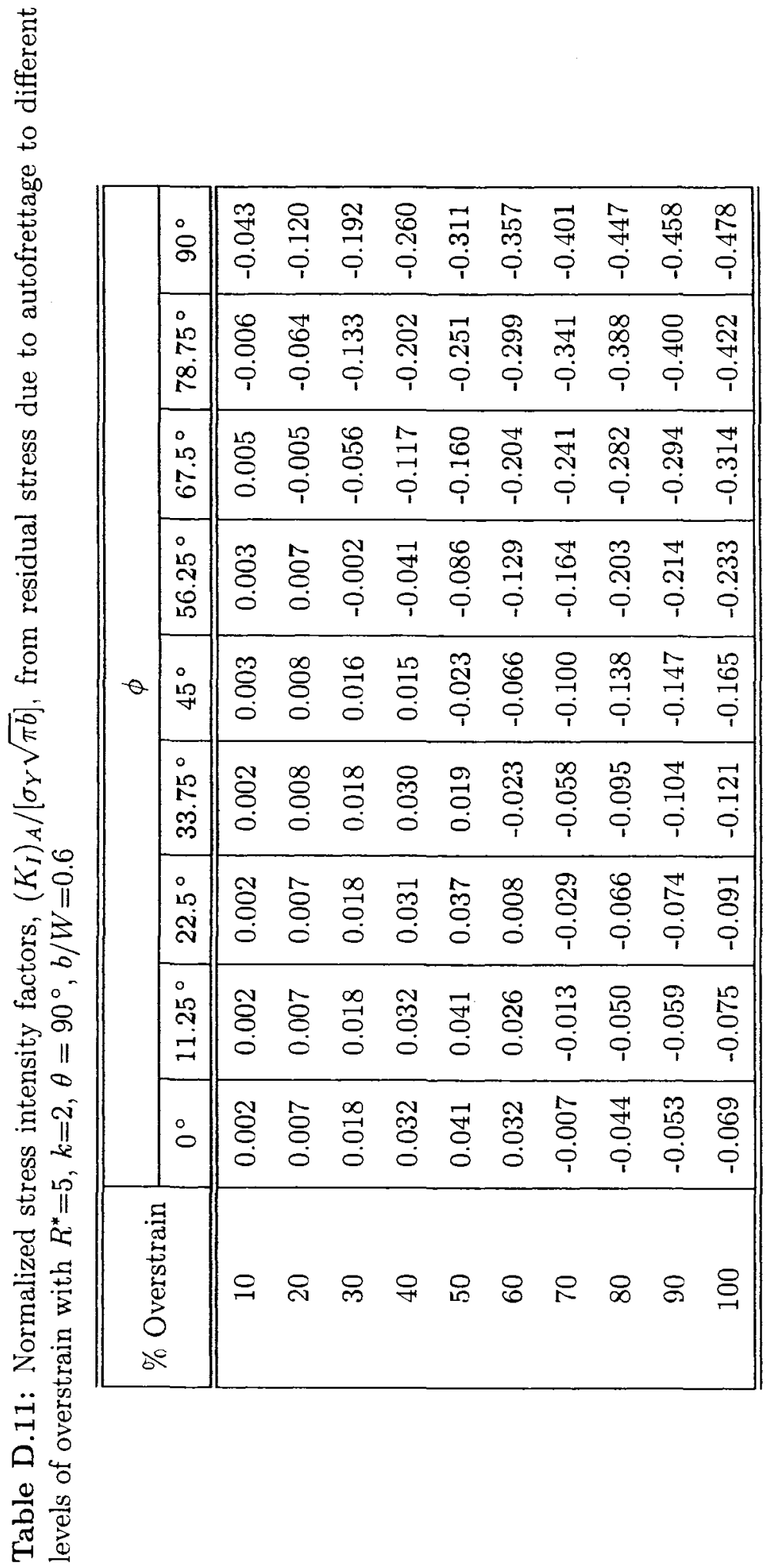




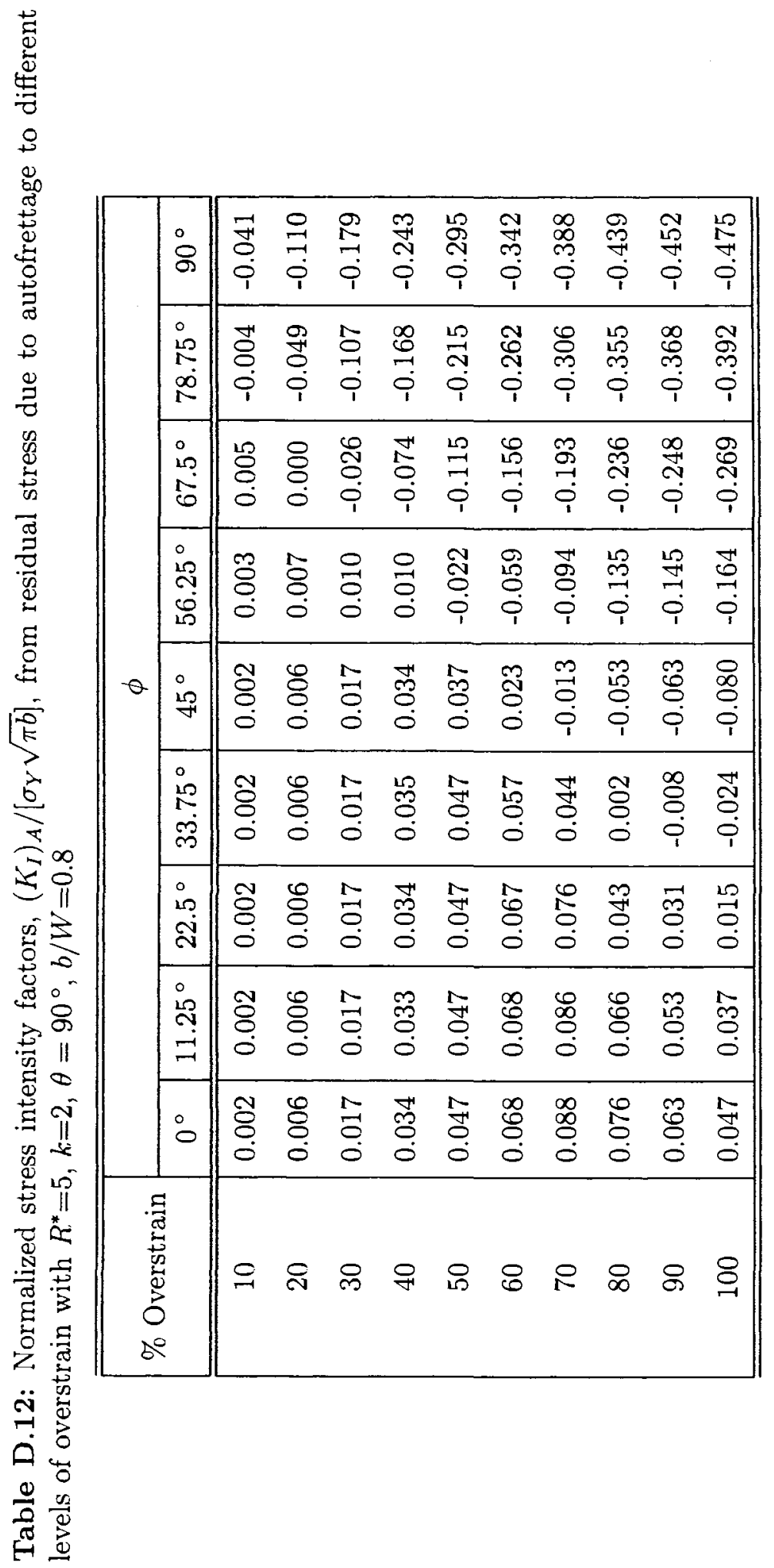




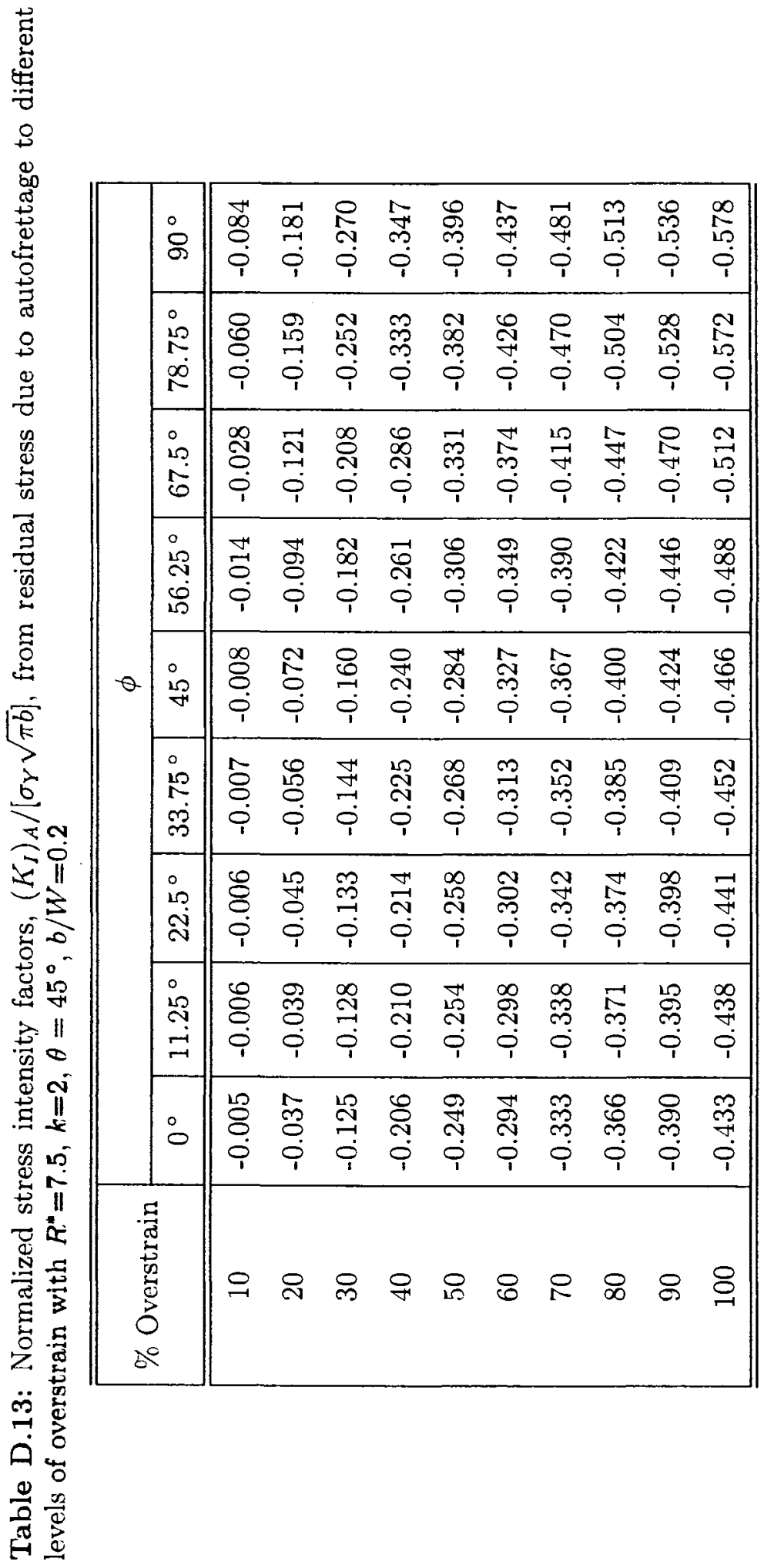




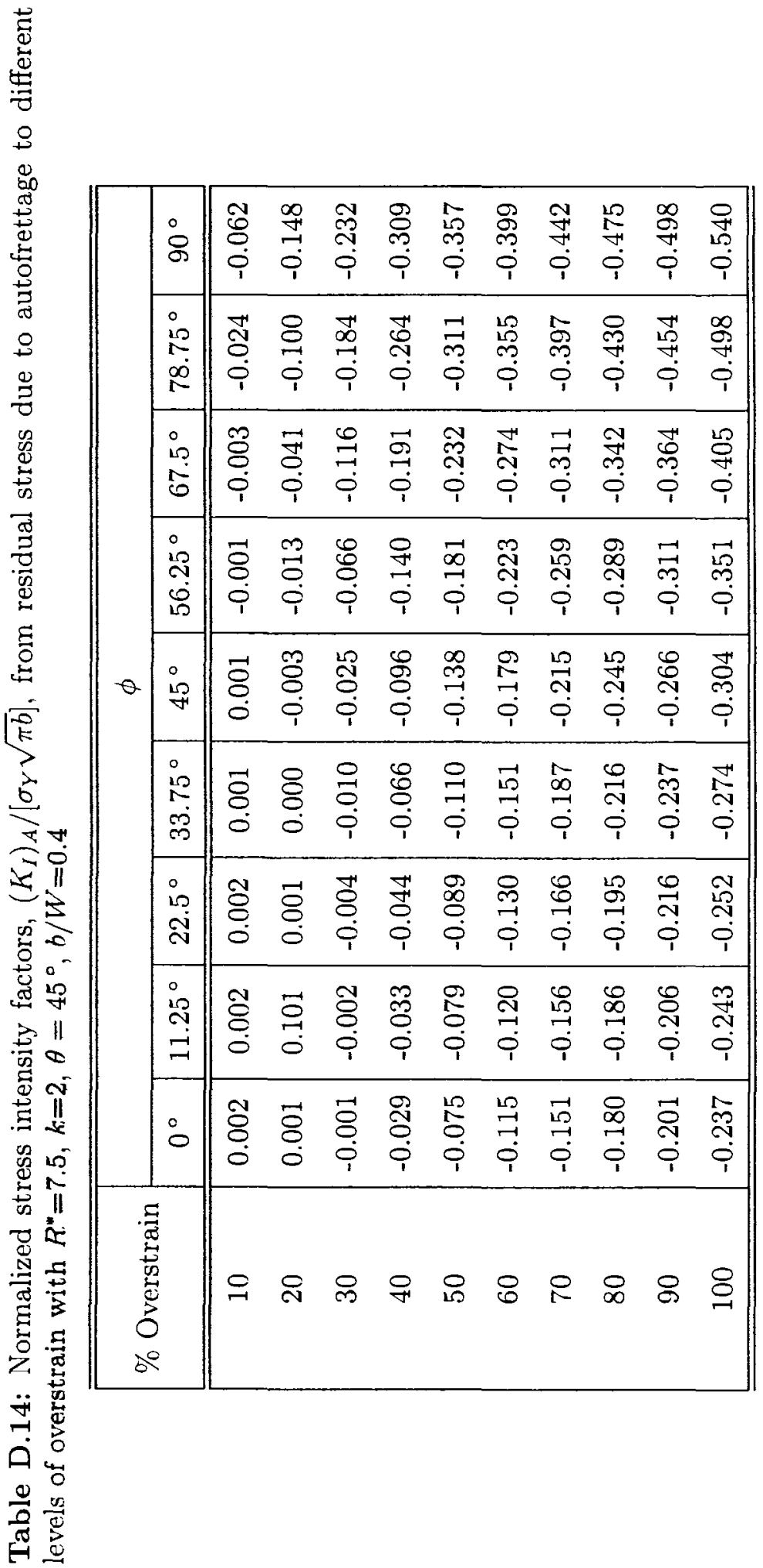




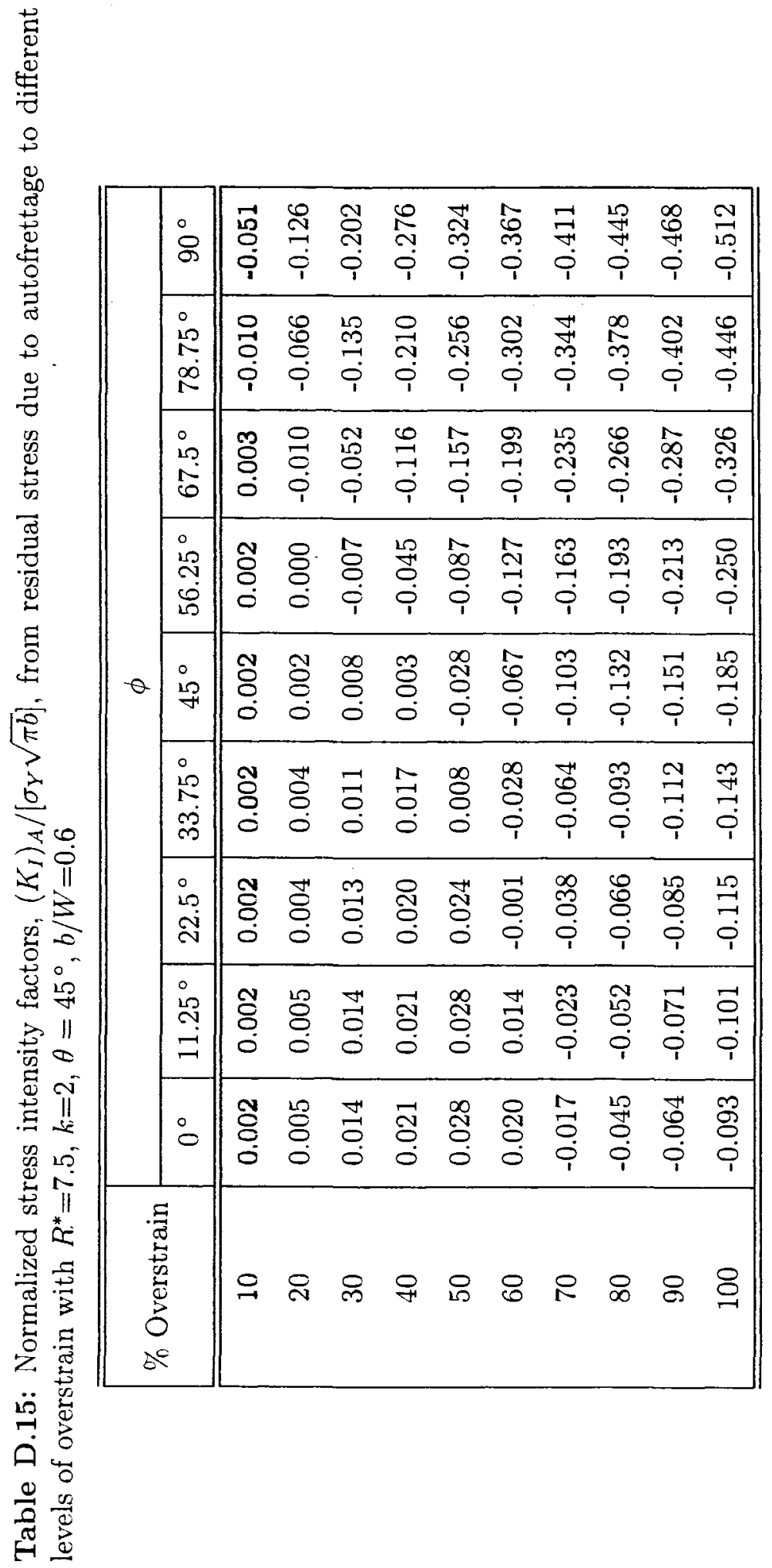




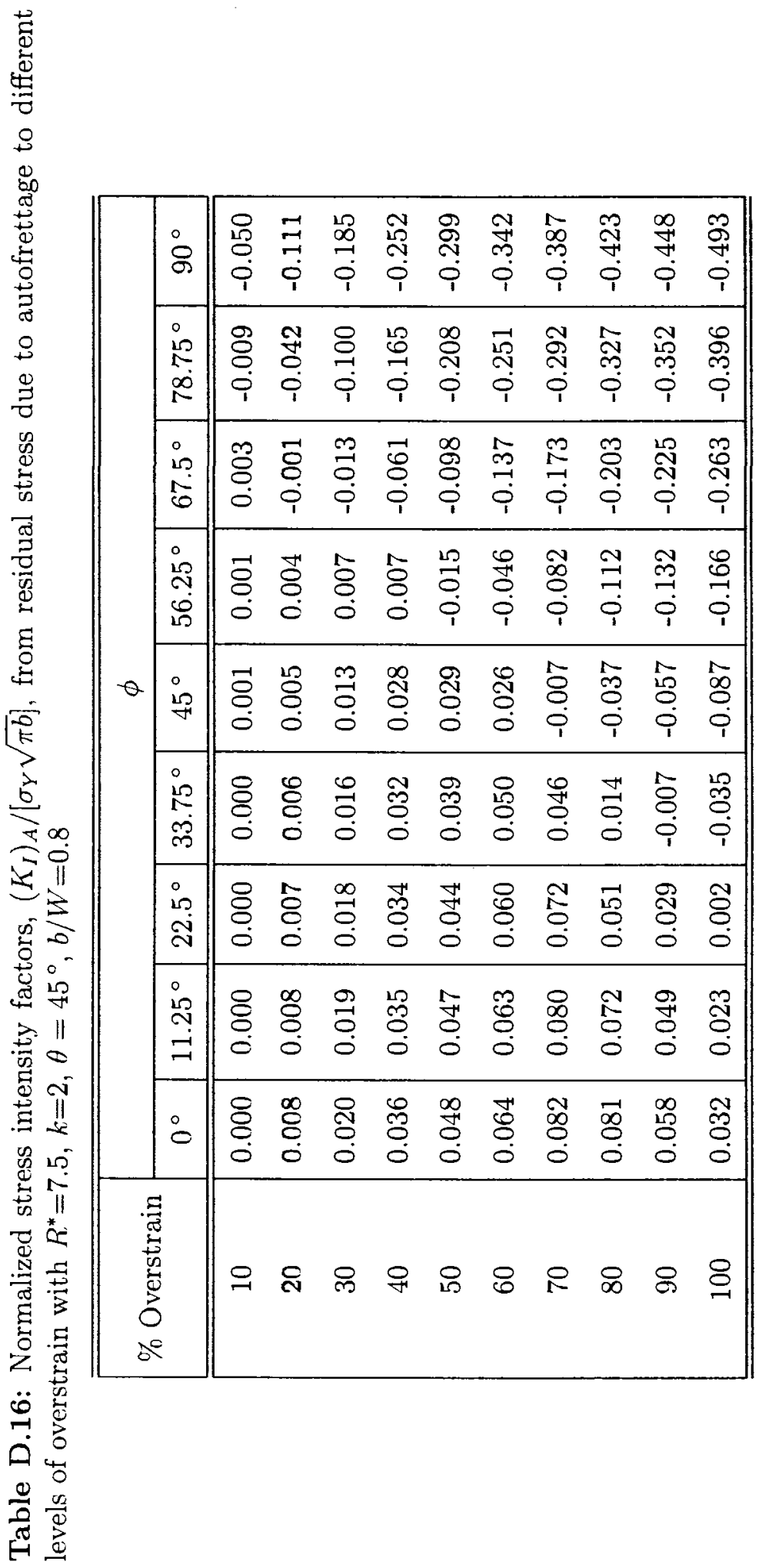




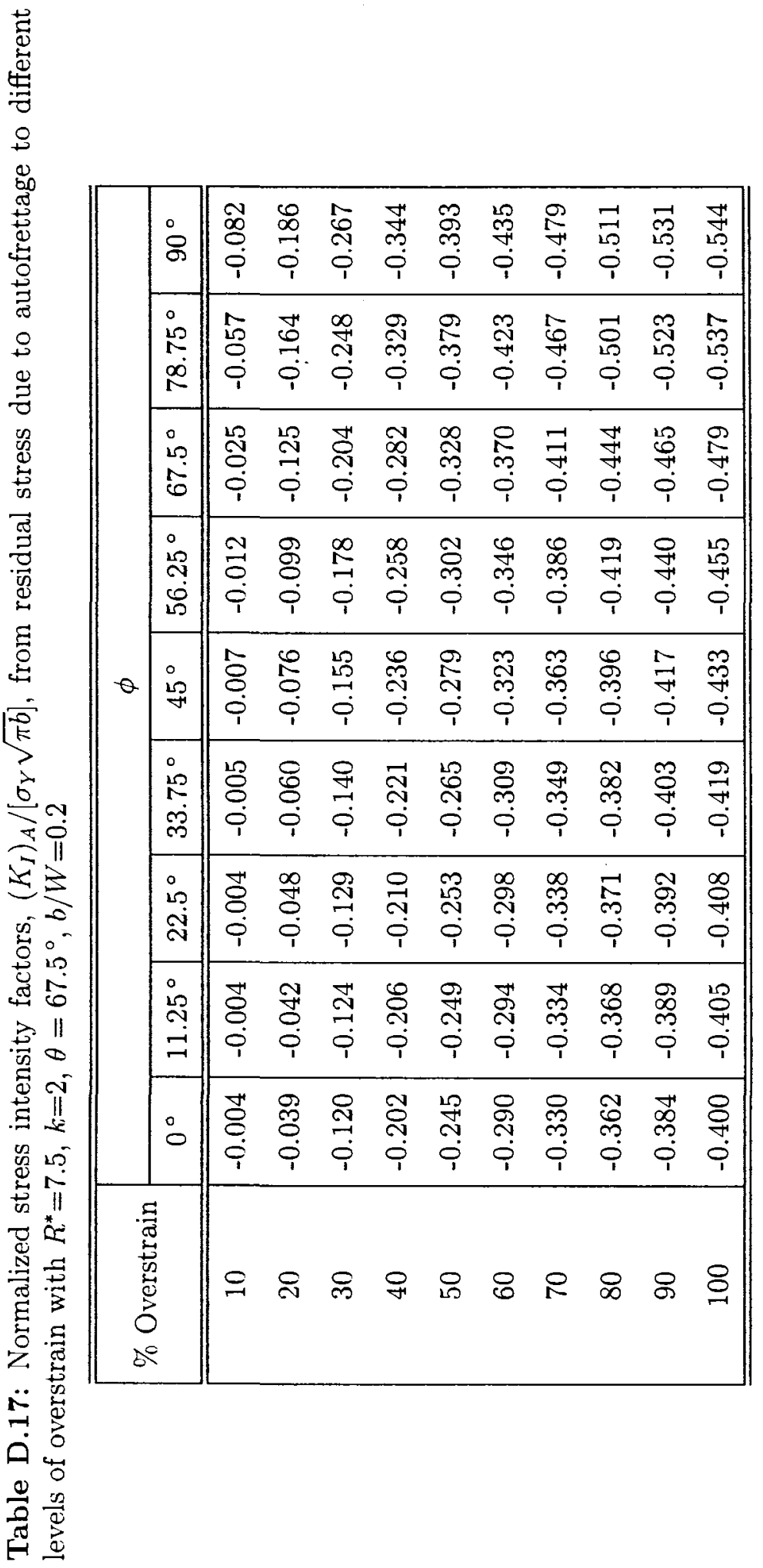




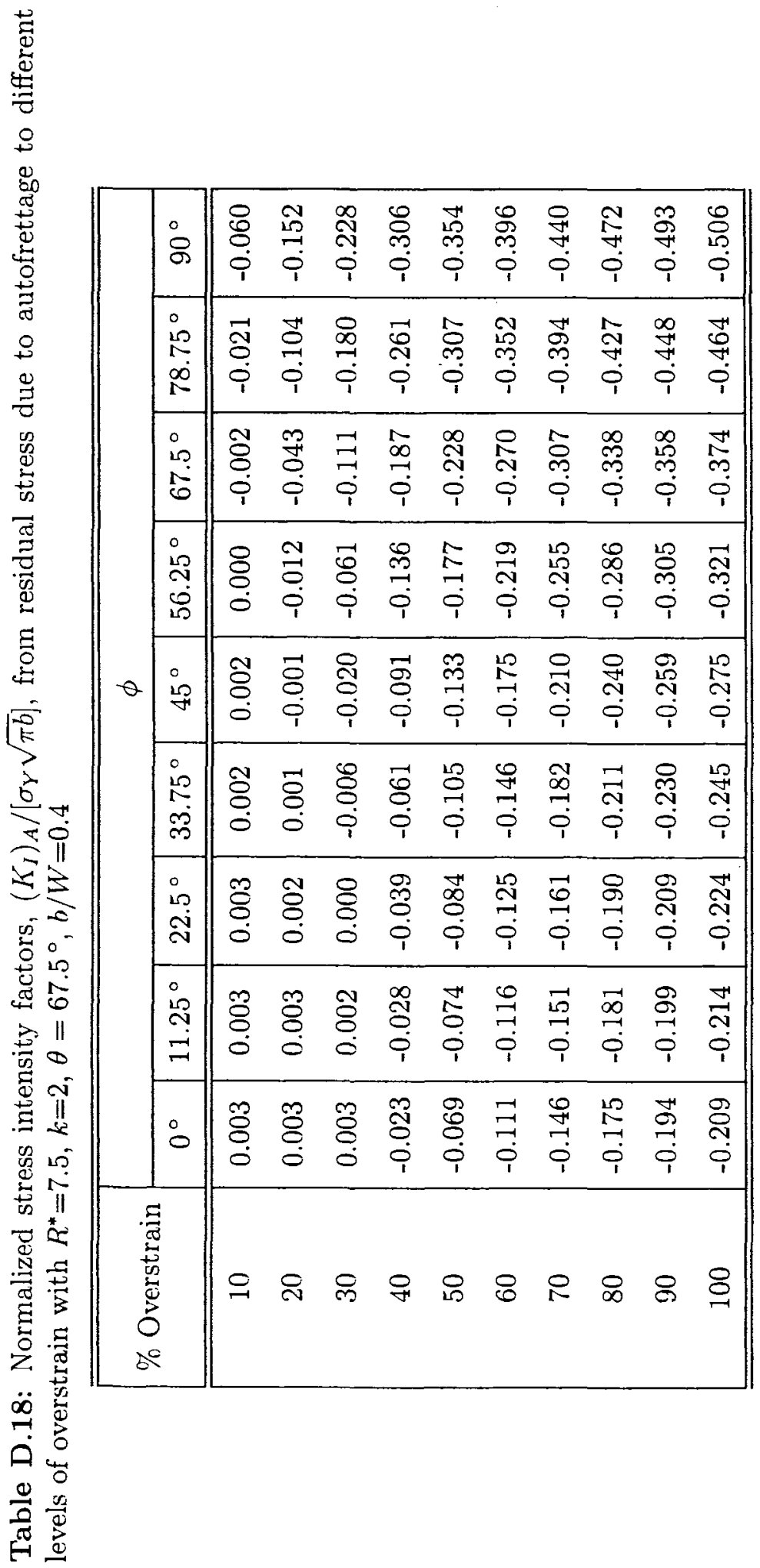




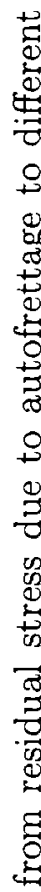

定

它

둥

$\leq$

की

응

疍

늘

品

壳 II

की 15

志

苋

콜

E्:

$\because$ 荡

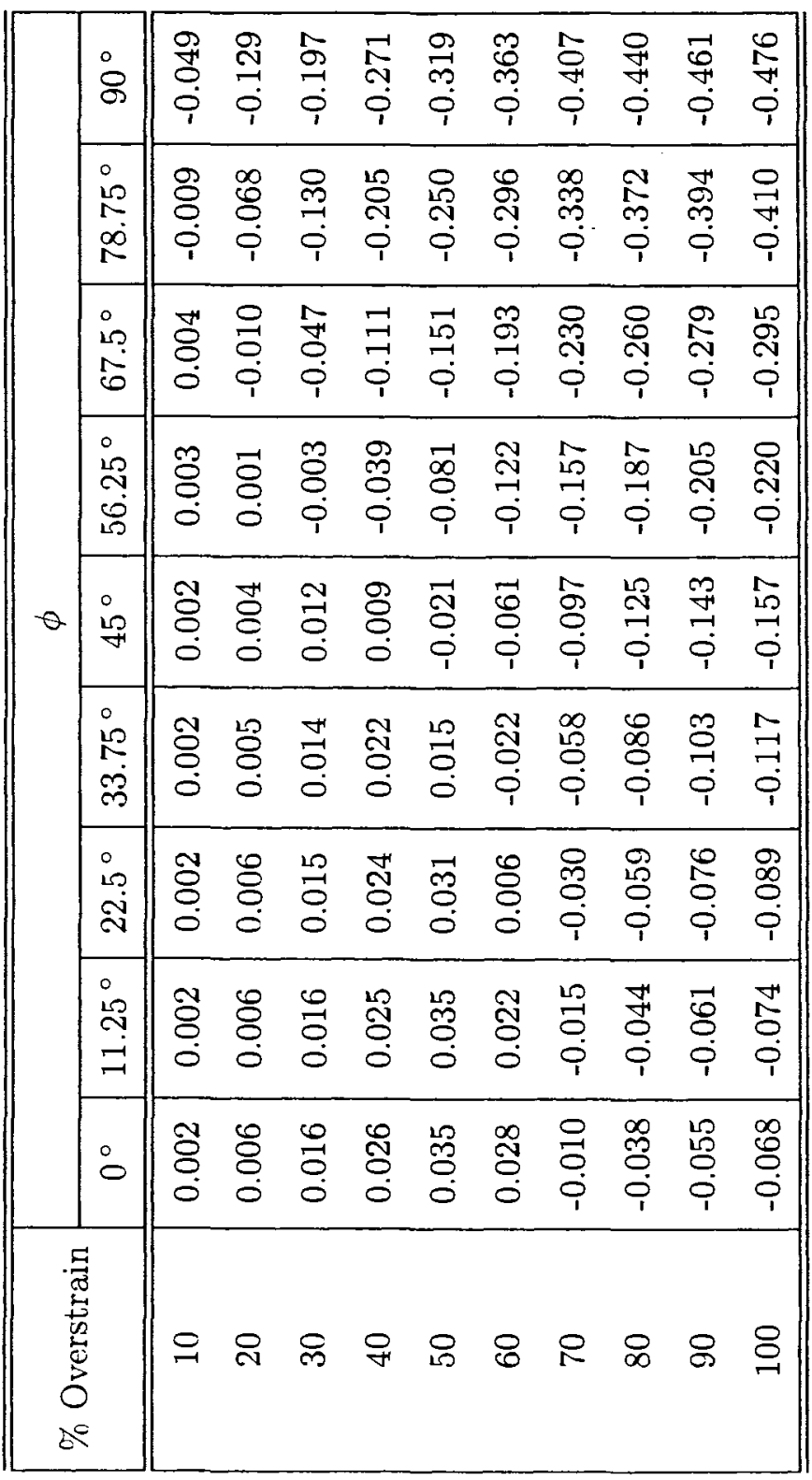

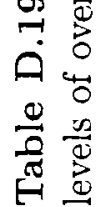




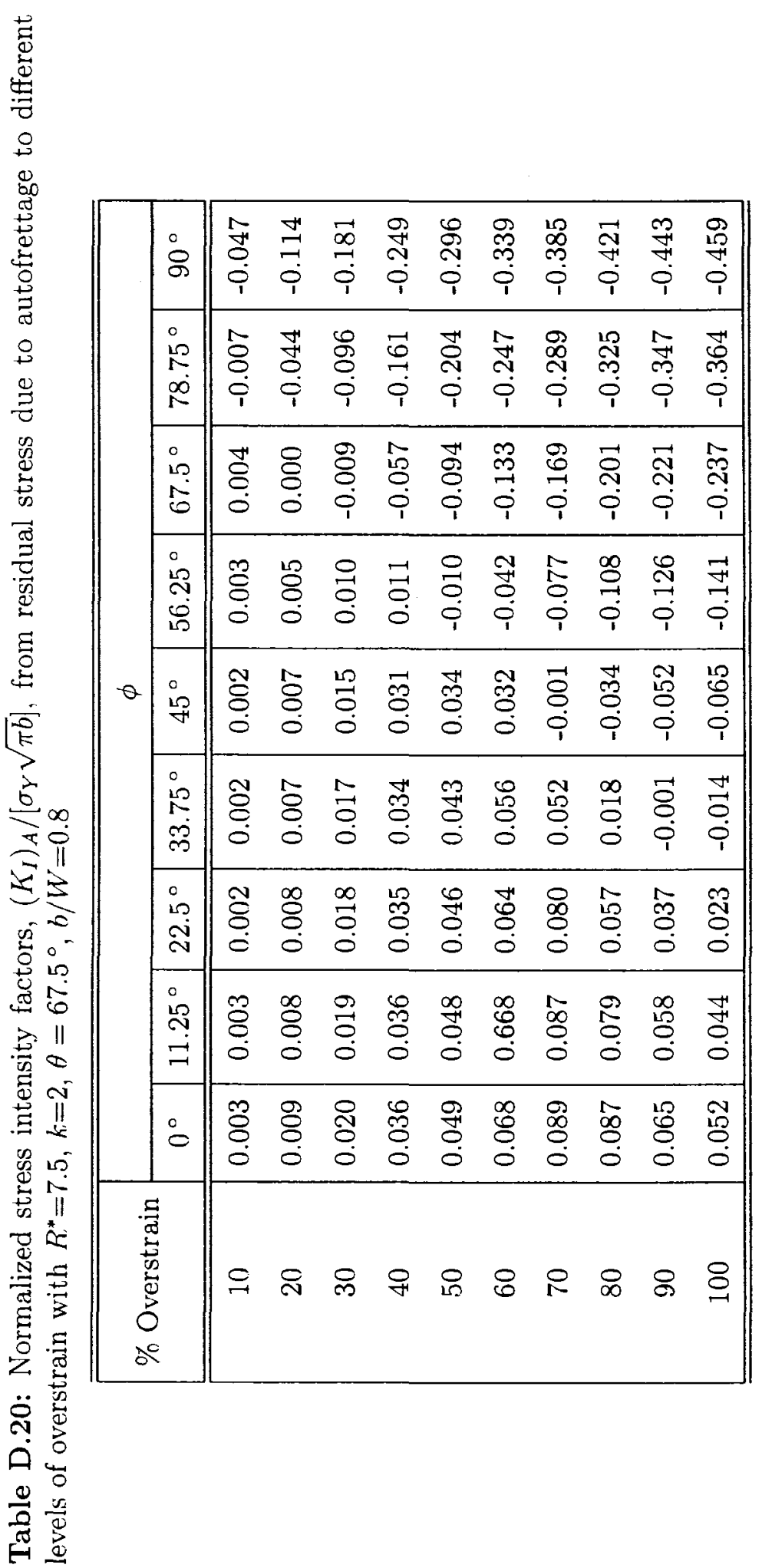




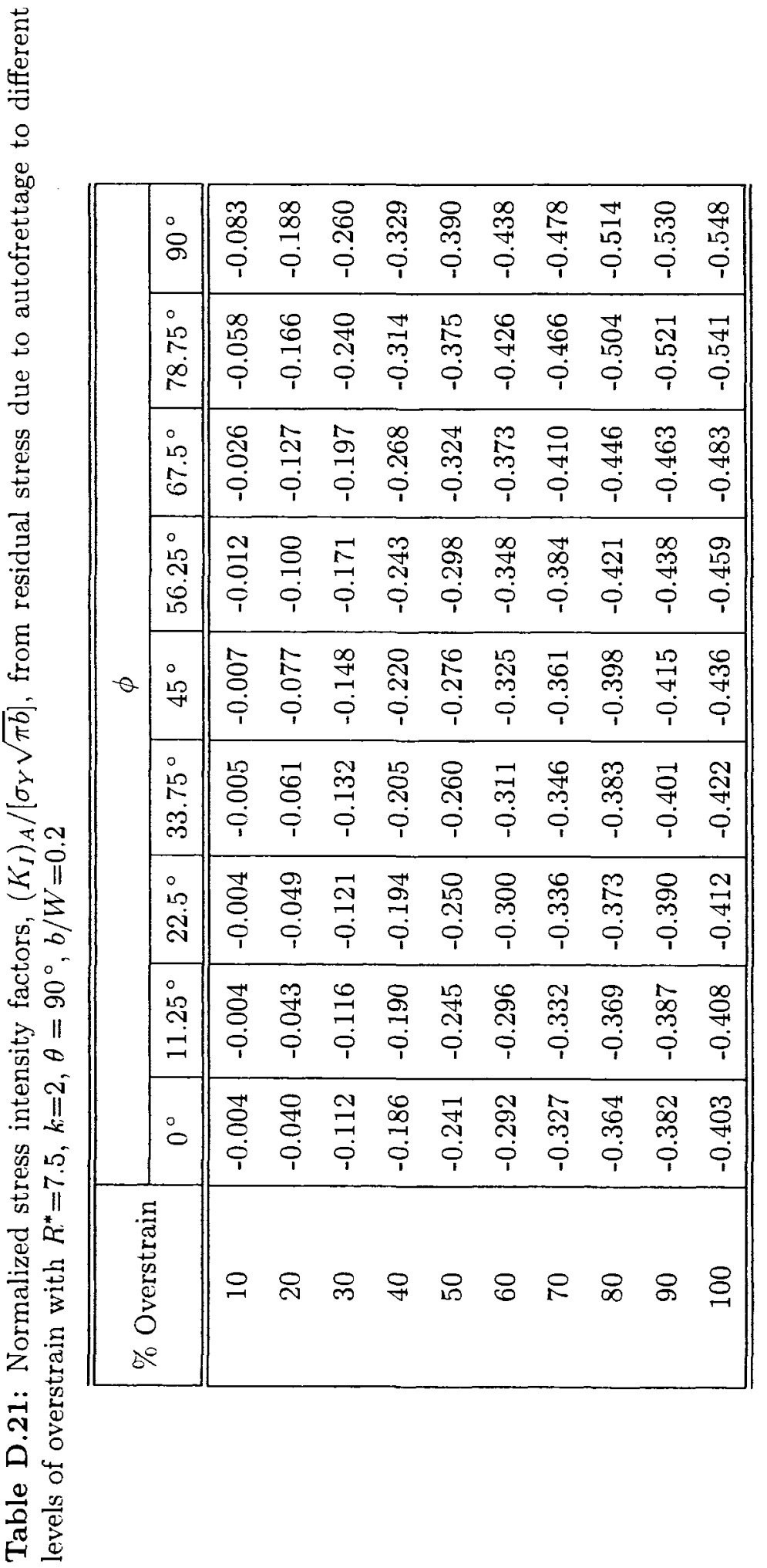




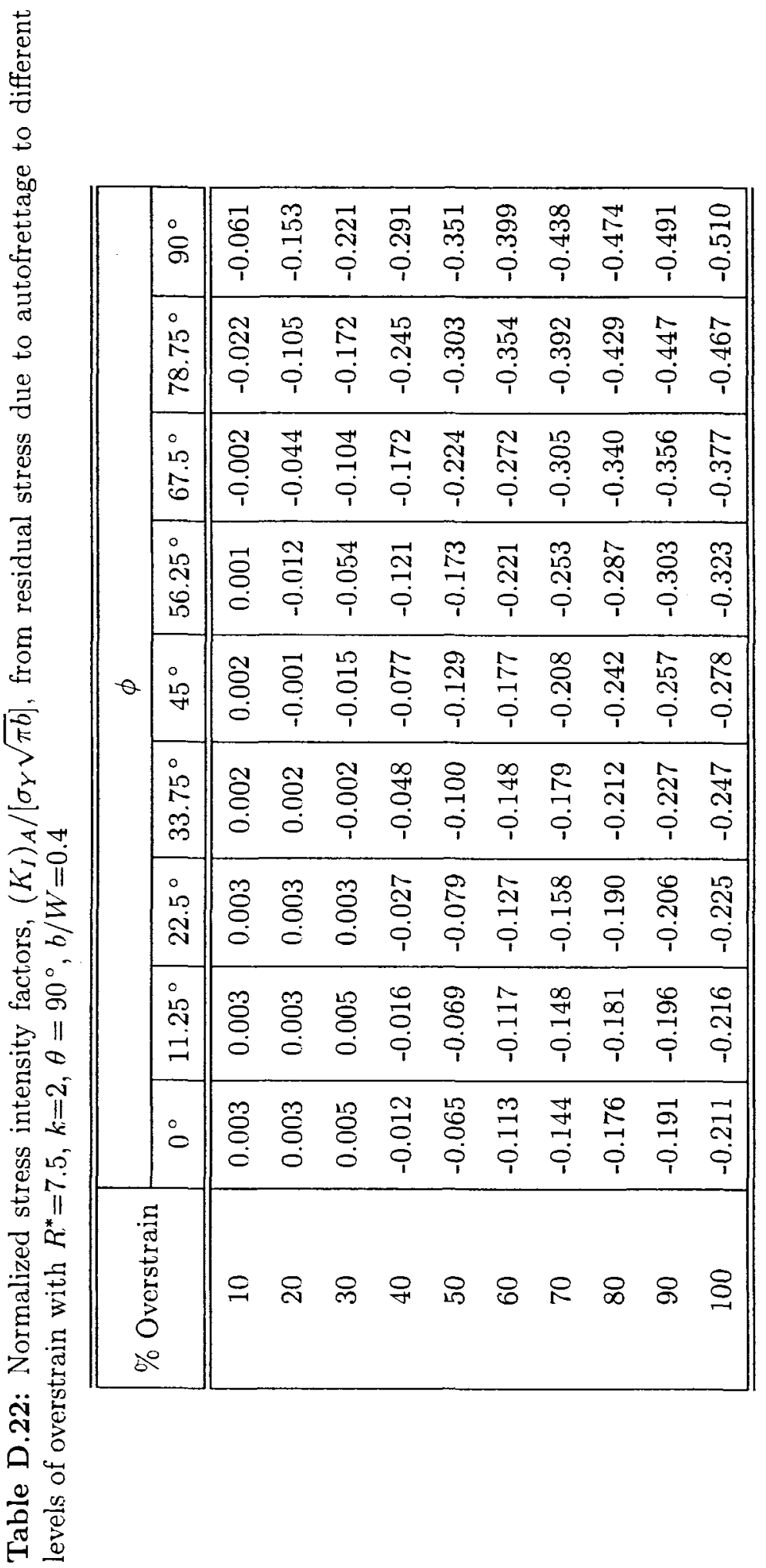




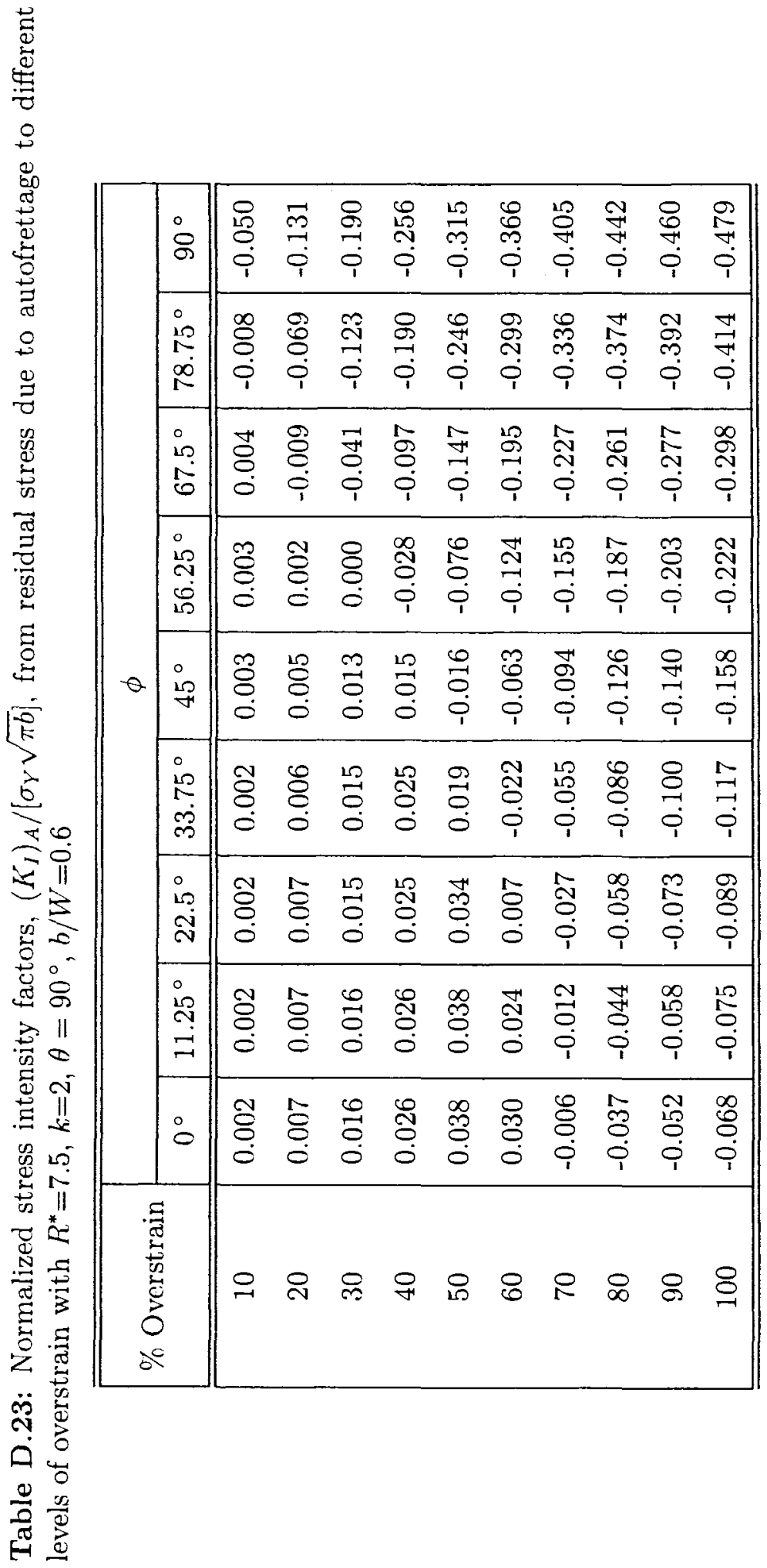




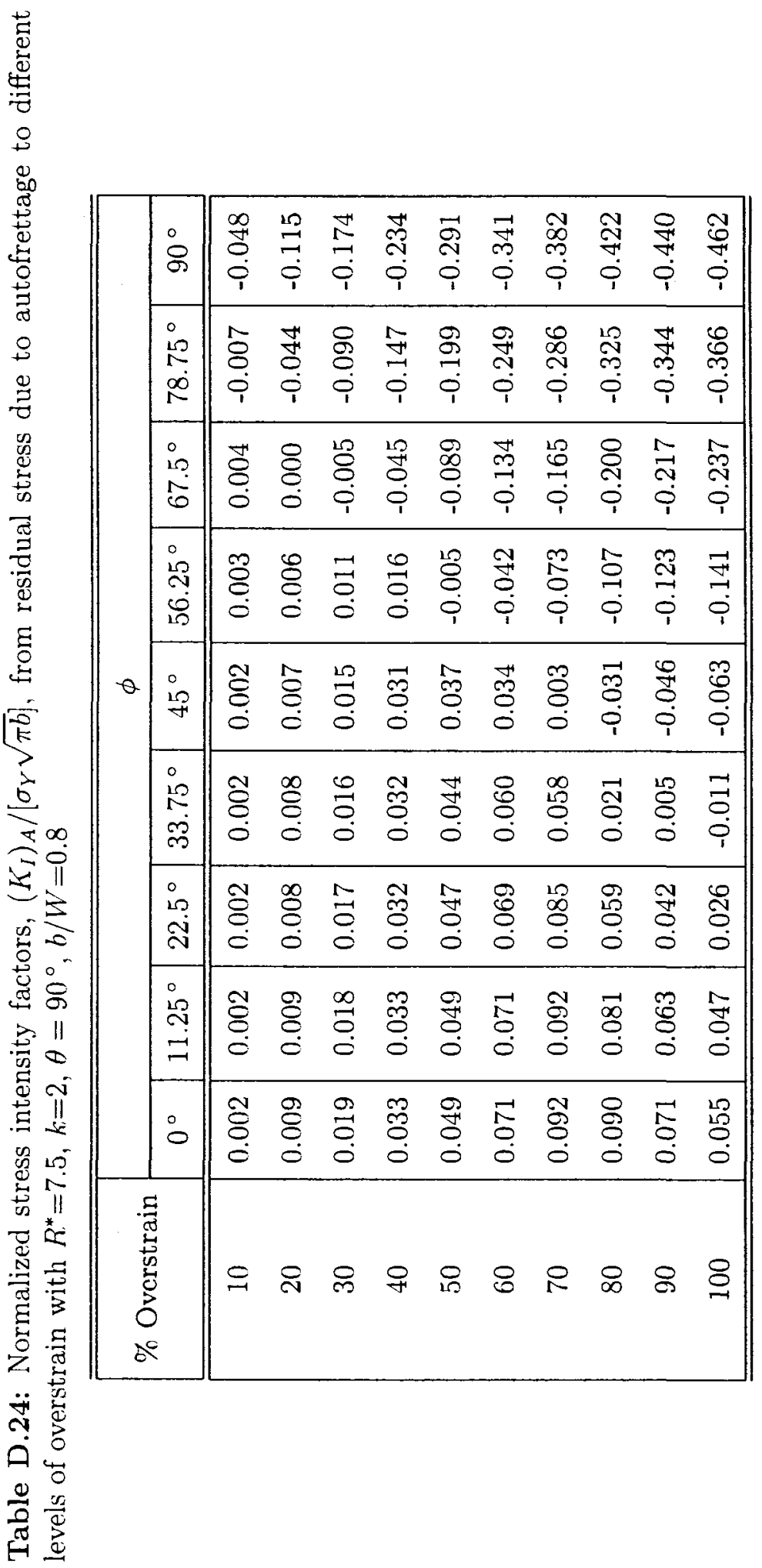




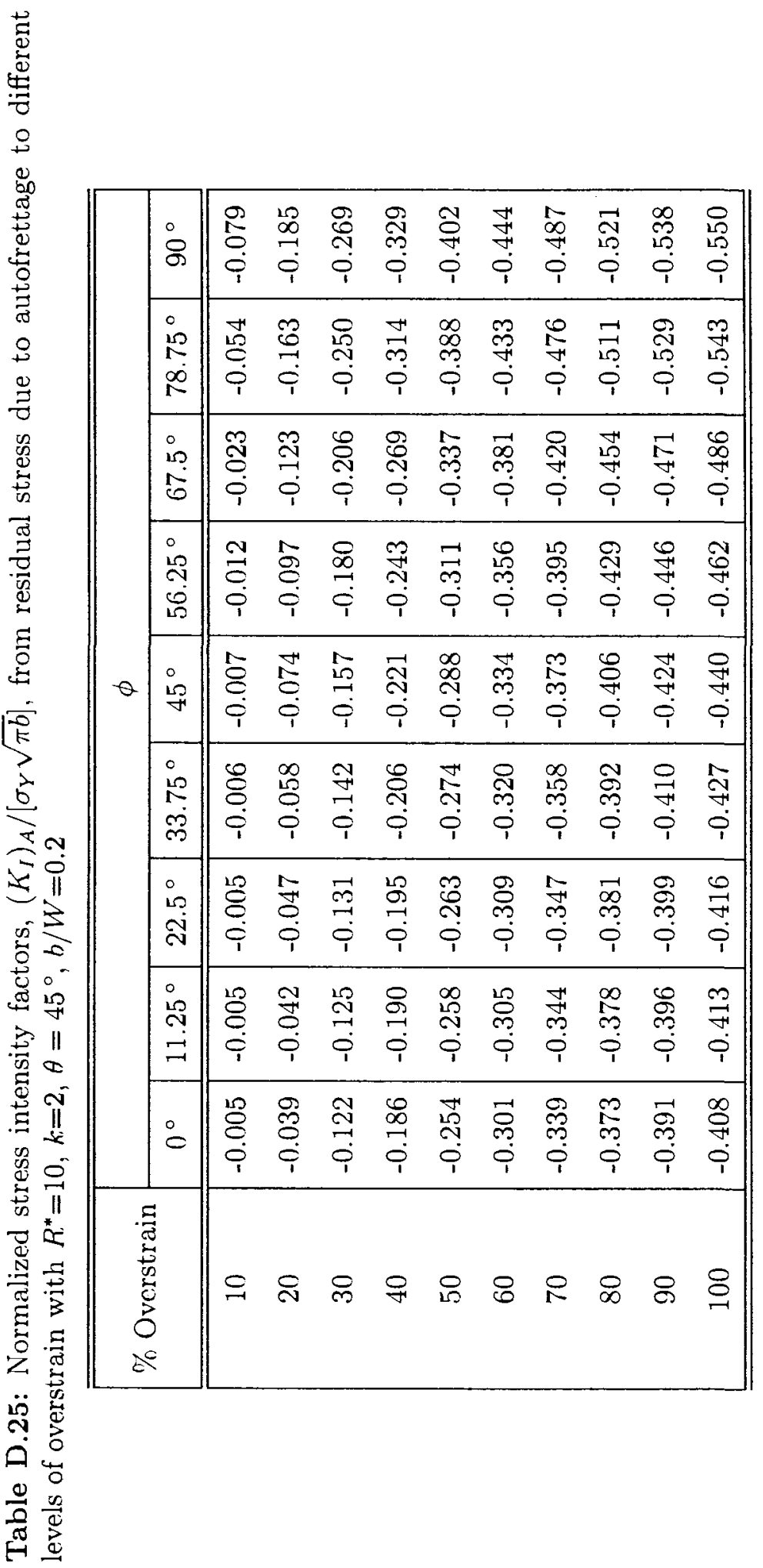




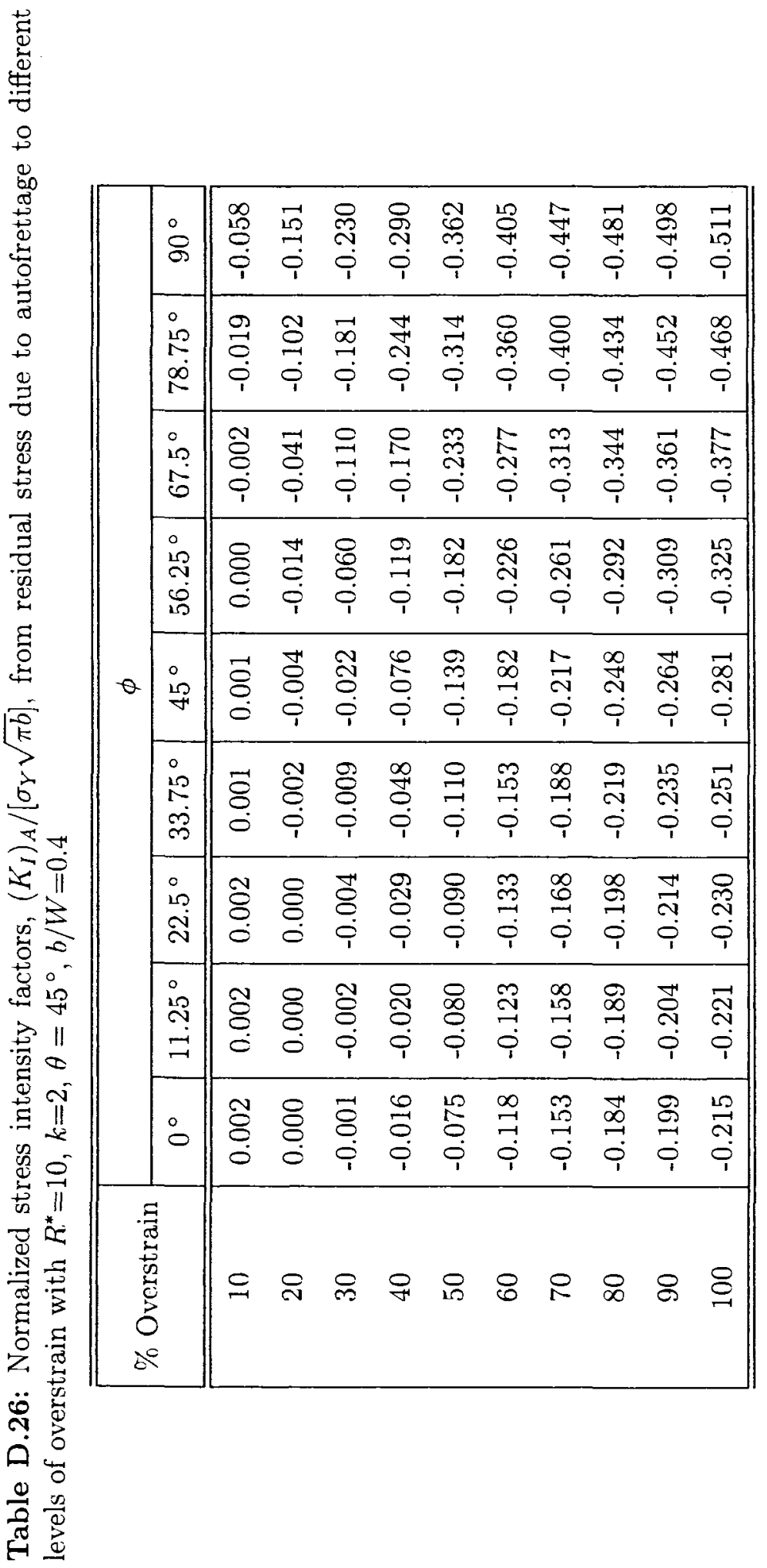




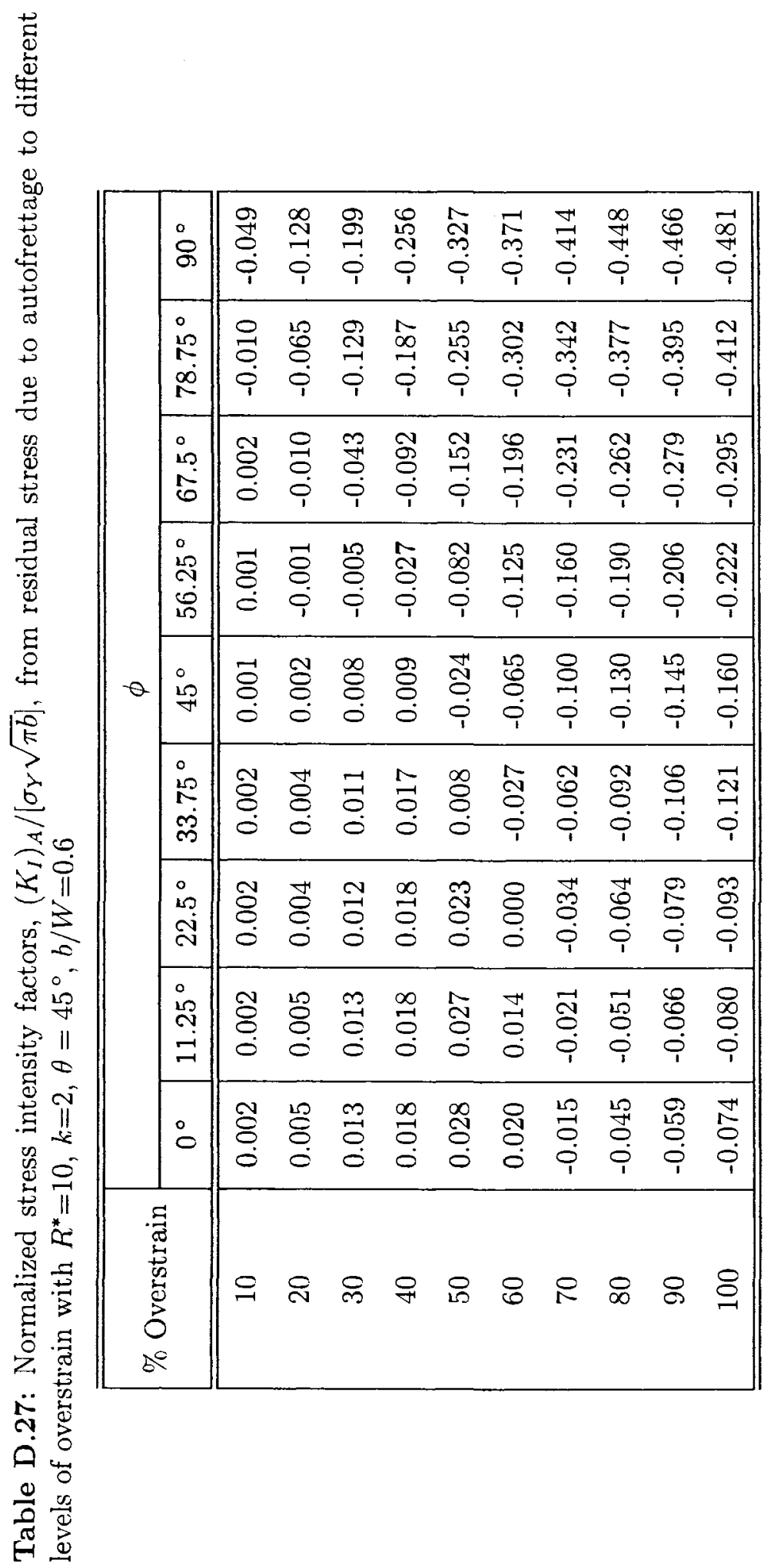




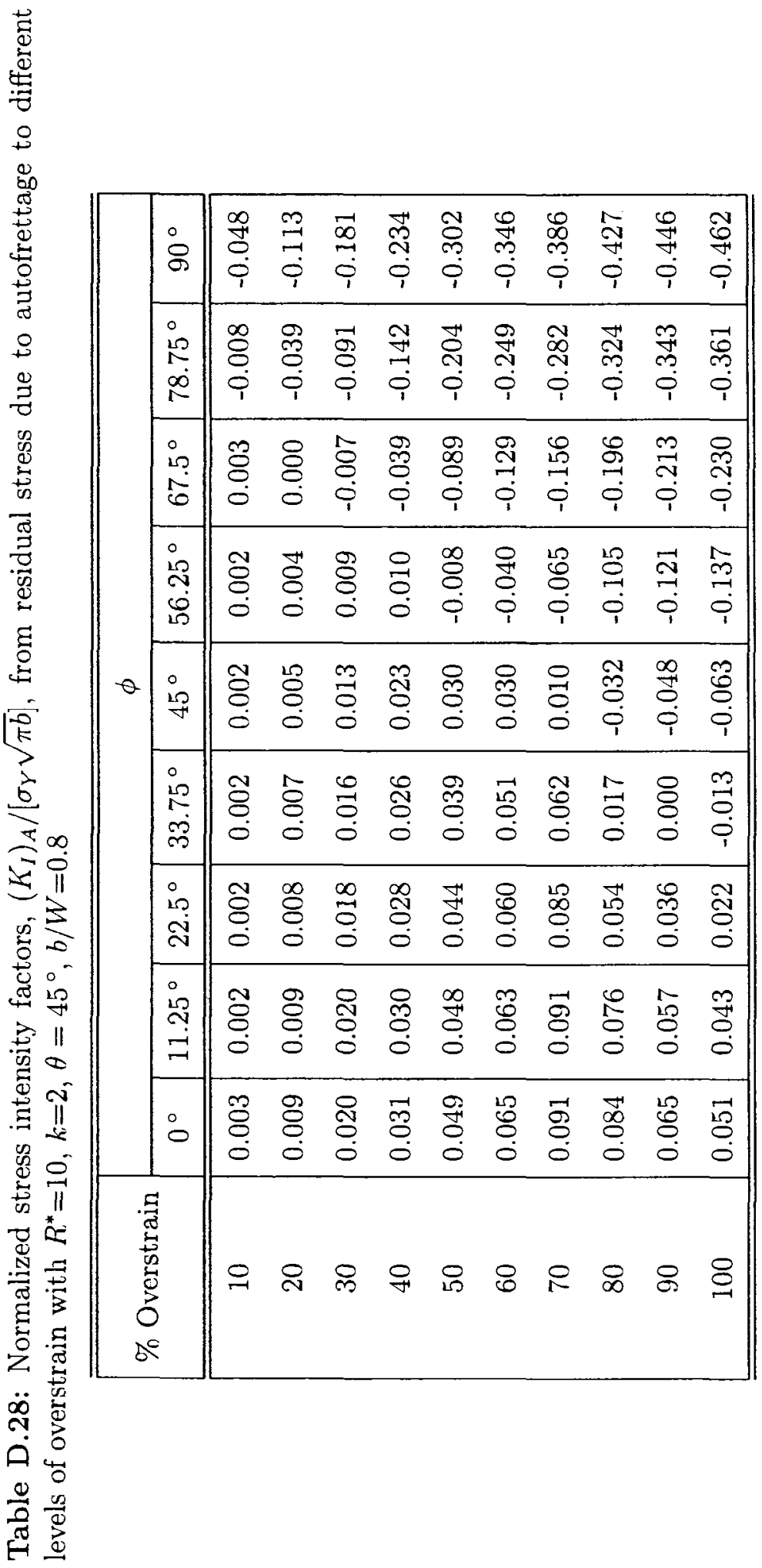




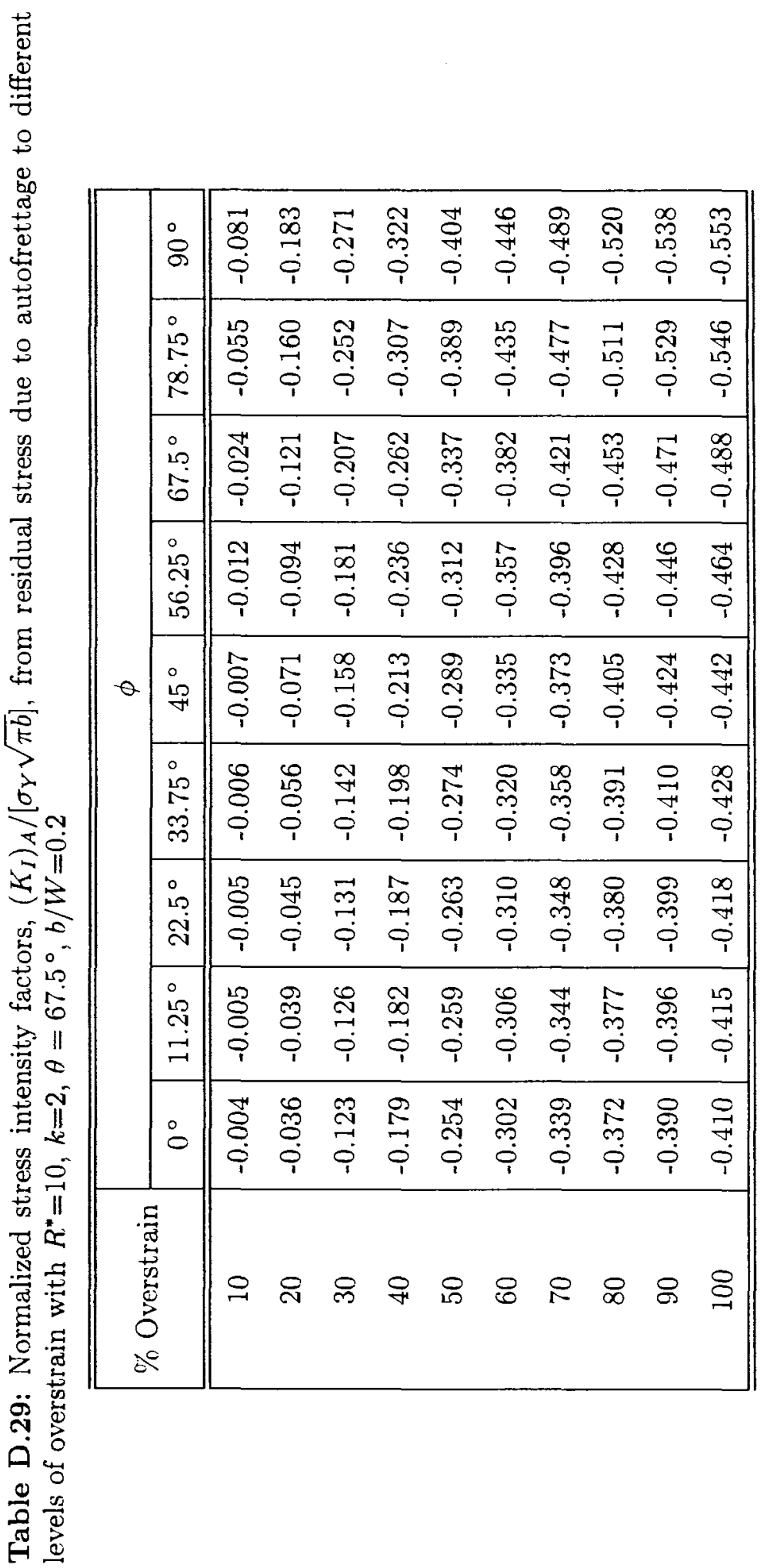




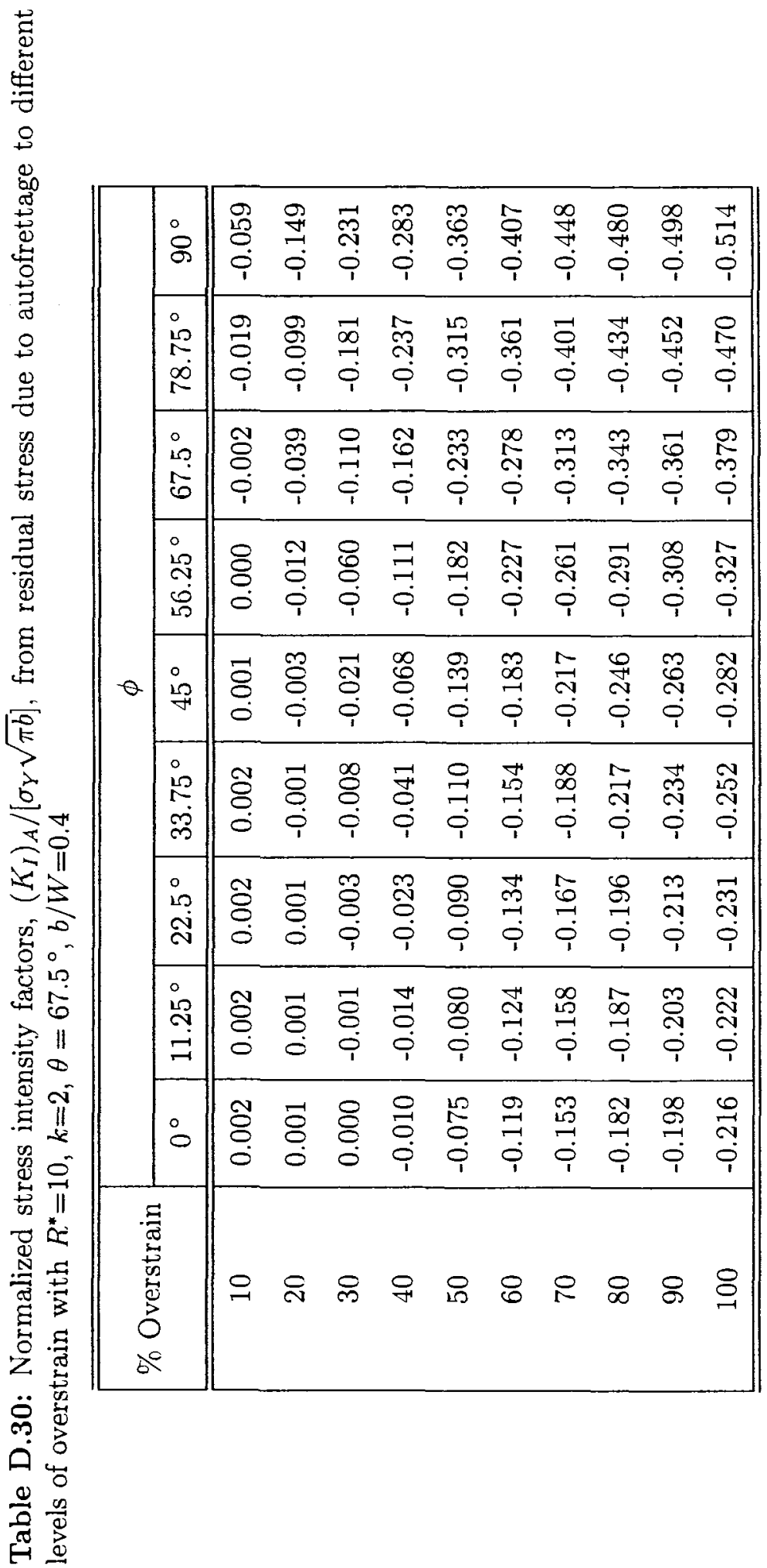




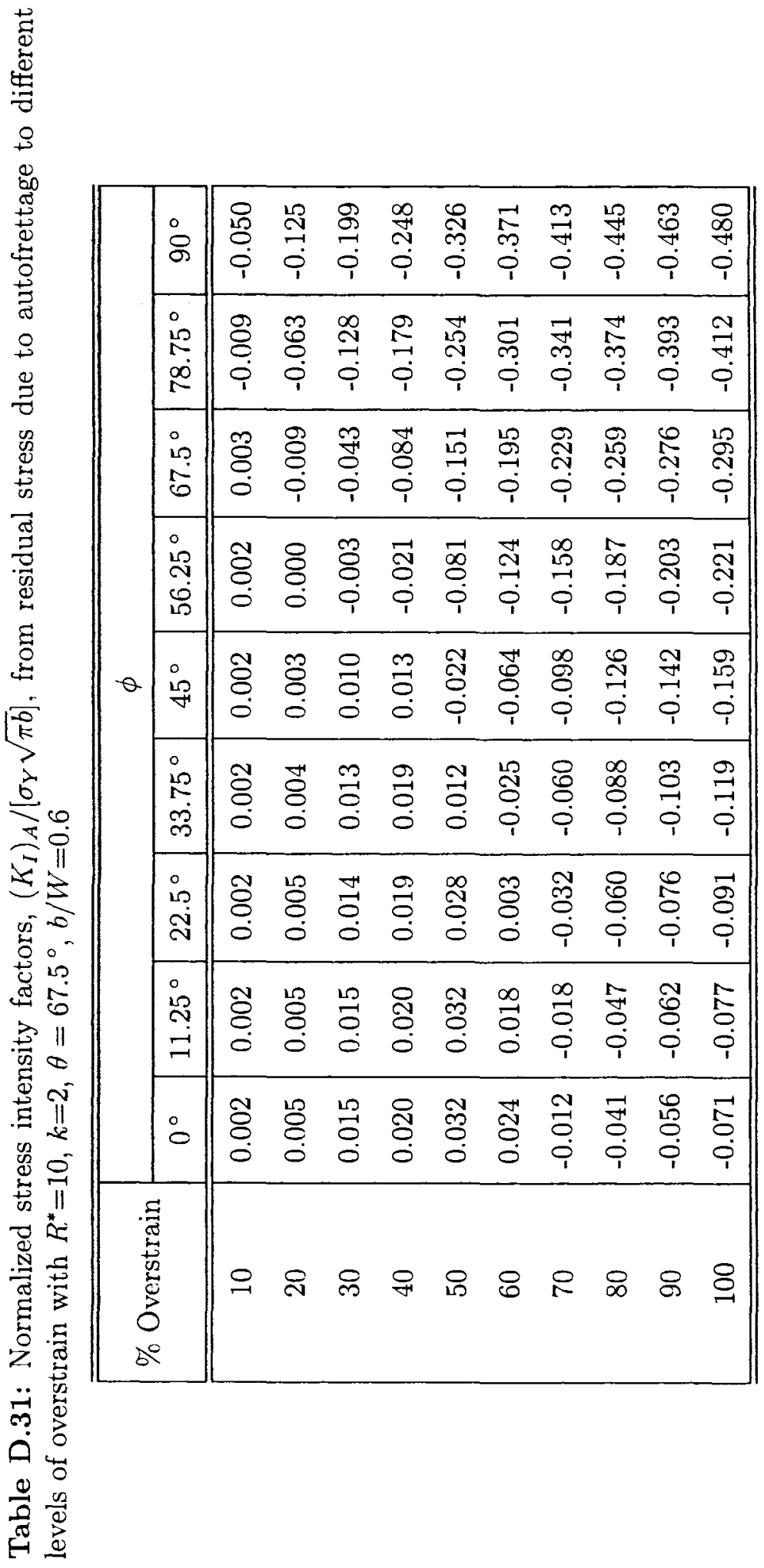




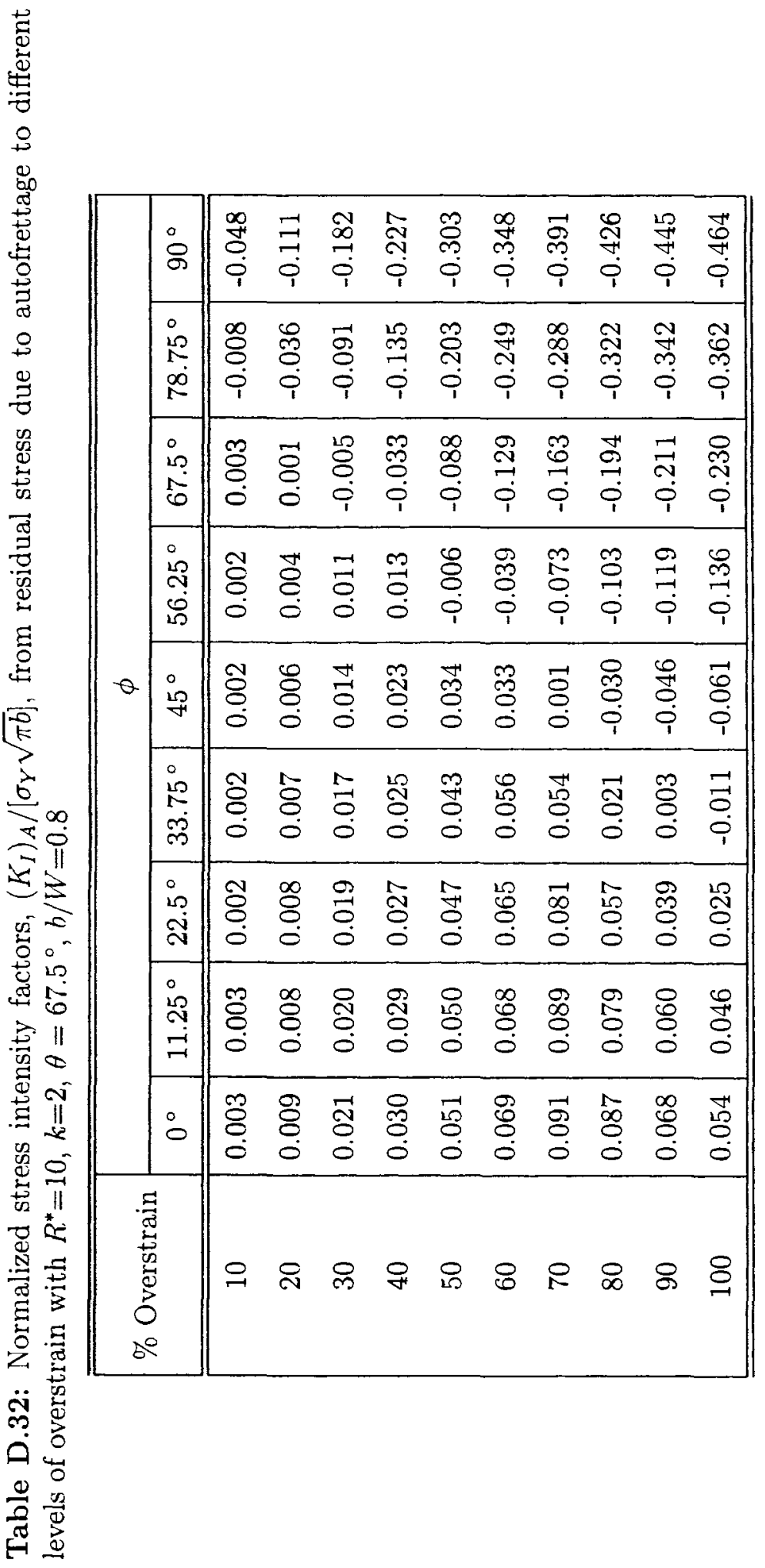




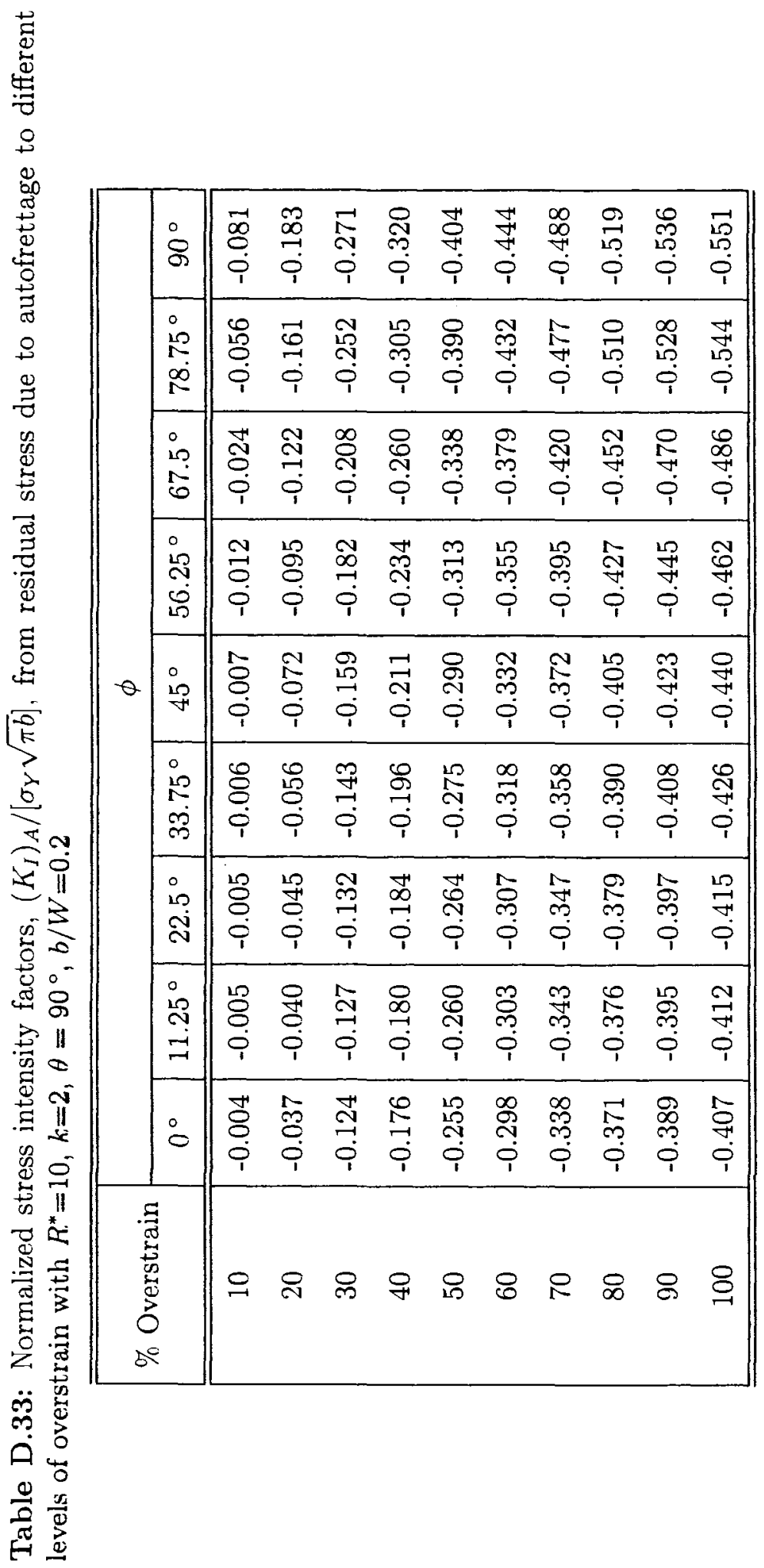




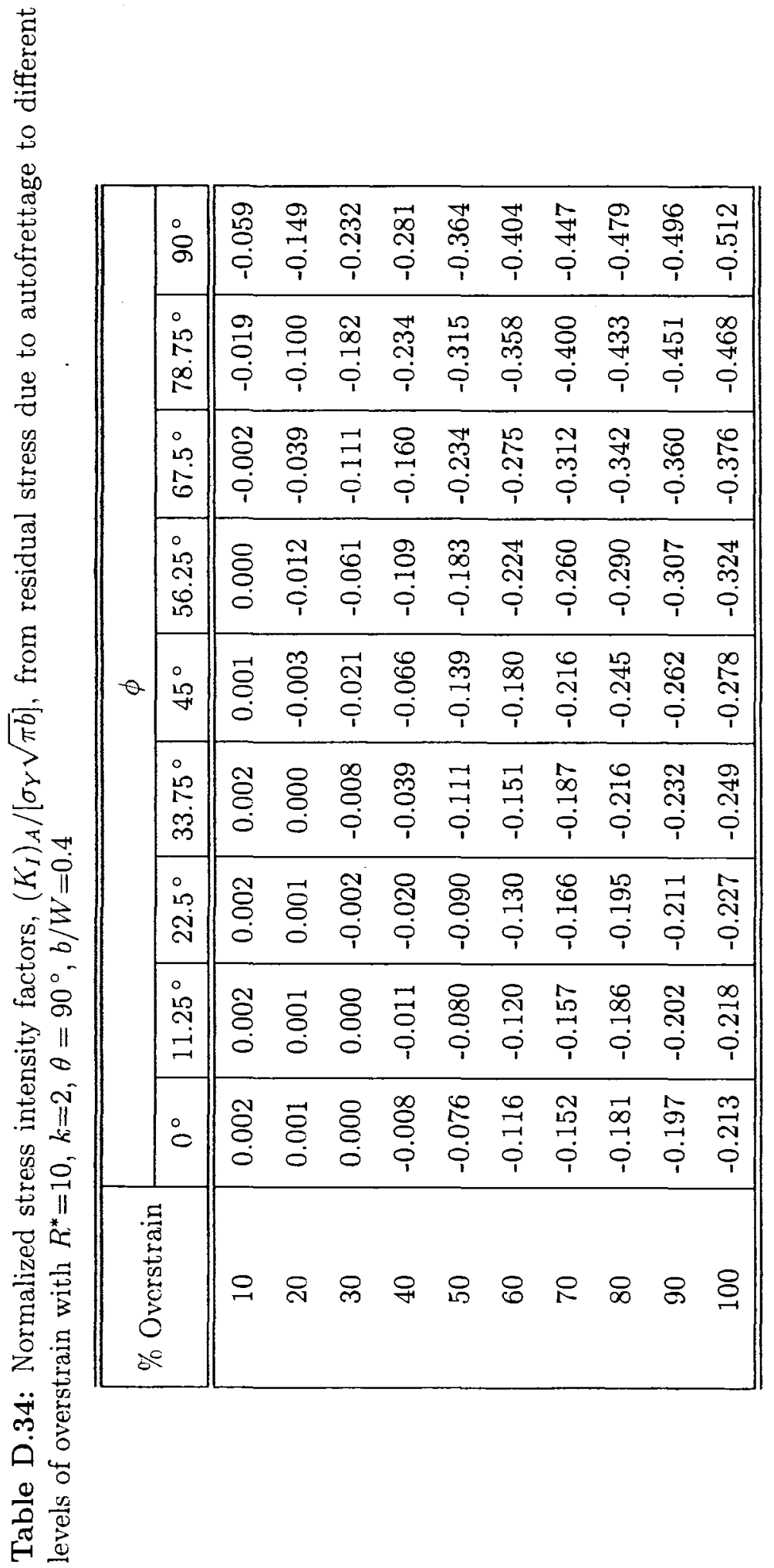




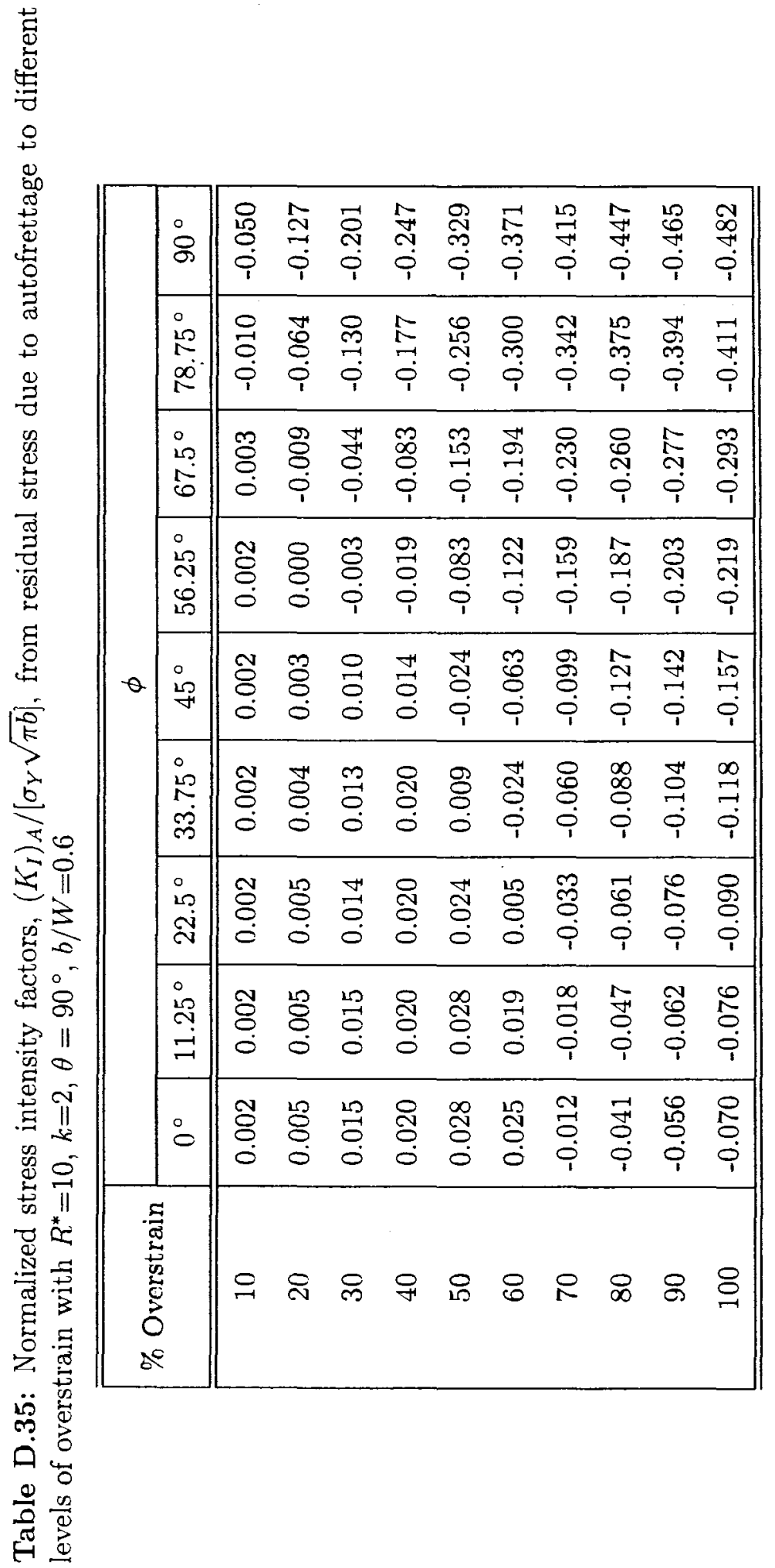




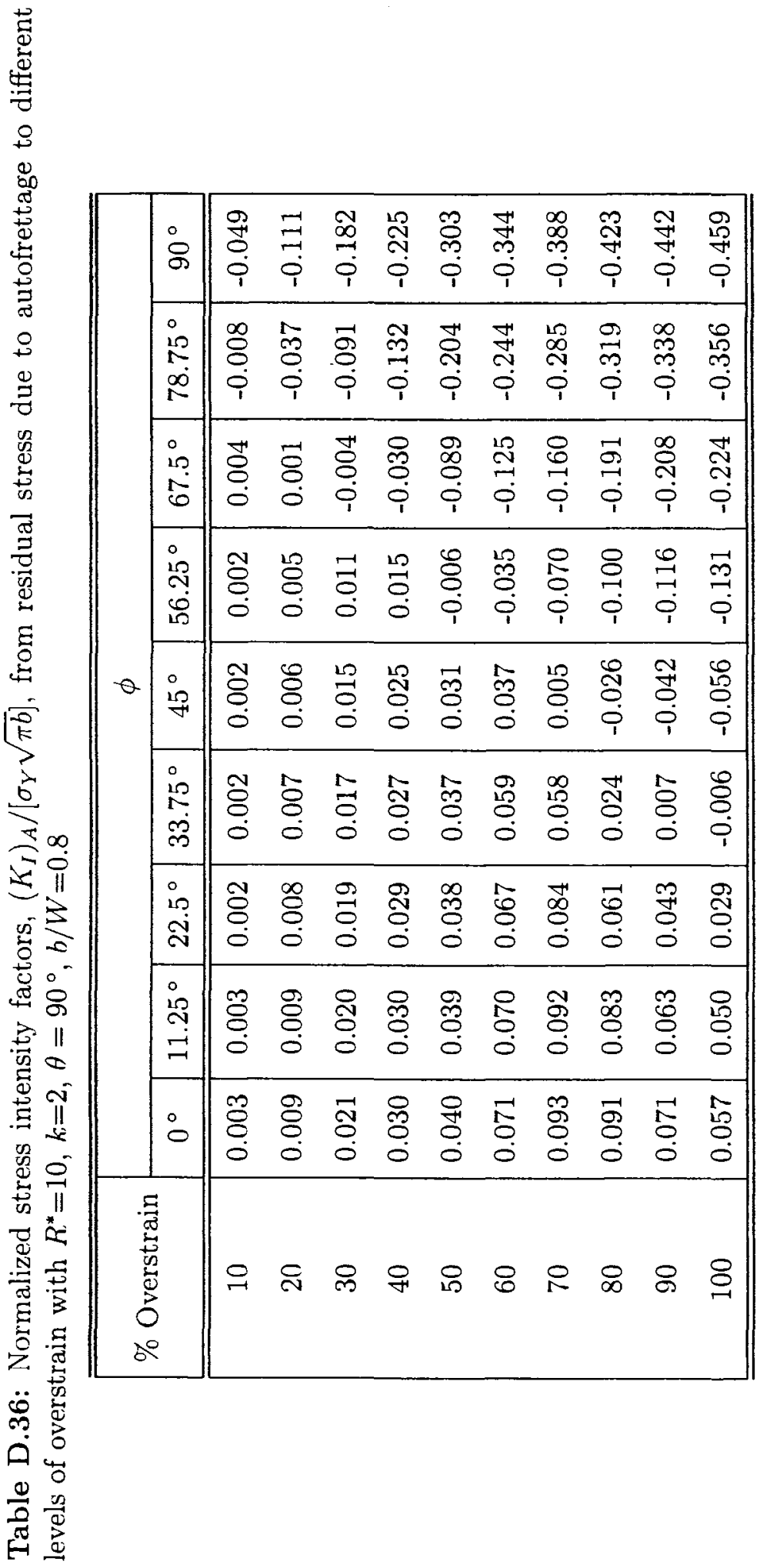

